\title{
Insertion of an intrinsically disordered domain in VelB supports selective heterodimer formation of fungal velvet domain regulatory proteins in Aspergillus nidulans
}

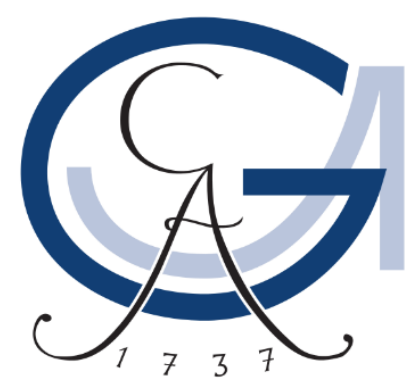

\begin{abstract}
Dissertation
for the award of the degree

"Doctor rerum naturalium"

of the Georg-August-Universität Göttingen
\end{abstract}

within the doctoral program "Microbiology and Biochemistry" of the Georg-August University School of Science (GAUSS)

submitted by

Sabine Thieme

from Eckernförde 


\section{Thesis Committee:}

Referee: $\quad$ Prof. Dr. Gerhard H. Braus

Department of Molecular Microbiology and Genetics, Georg-August-Universität Göttingen

$2^{\text {nd }}$ referee: Prof. Dr. Ralf Ficner

Department of Structural Biology, Georg-August-Universität Göttingen

$3^{\text {rd }}$ referee: Prof. Dr. Stefanie Pöggeler

Department of Genetics and Eukaryotic Microorganisms, Georg-August-Universität Göttingen

\section{Further members of the examination board:}

Prof. Dr. Heike Krebber

Department of Molecular Genetics, Georg-August-Universität Göttingen Prof. Dr. Rolf Daniel

Department of Genomic and Applied Microbiology, Georg-August-Universität Göttingen Jun.-Prof. Dr. Kai Heimel

Department of Molecular Microbiology and Genetics, Georg-August-Universität, Göttingen

Date of oral examination: April $12^{\text {th }}, 2018$ 


\section{Declaration of independence}

Herewith I declare that the dissertation entitled "Insertion of an intrinsically disordered domain in VelB supports selective heterodimer formation of fungal velvet domain regulatory proteins in Aspergillus nidulans" was written on my own and independently without any other aids and sources than indicated.

Sabine Thieme

Göttingen, 2018 
This work was conducted in the group of Prof. Dr. Gerhard H. Braus at the Department of Molecular Microbiology and Genetics, Institute of Microbiology and Genetics, Georg-AugustUniversität Göttingen.

Part of this work will be published in:

Sabine Thieme, Jennifer Gerke, Christoph Sasse, Oliver Valerius, Karl G. Thieme, Antje K. Heinrich, Helge B. Bode and Gerhard H. Braus (2018). Insertion of an intrinsically disordered domain in VelB supports selective heterodimer formation of fungal velvet domain regulatory proteins in Aspergillus nidulans. In preparation. 


\section{Table of contents}

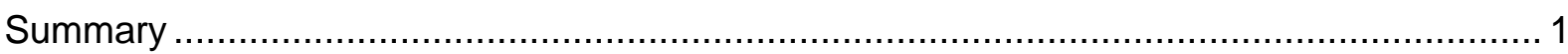

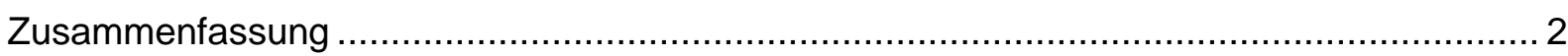

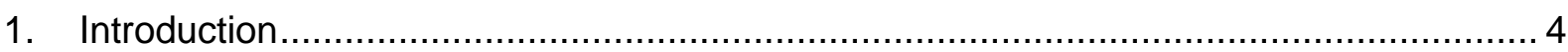

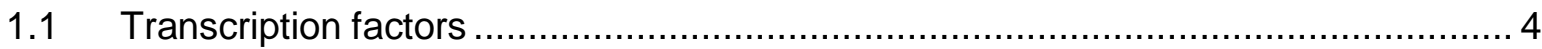

1.1.1 Transcriptional regulation of target genes by dimeric complexes .................... 4

1.2 Aspergillus nidulans - a reference organism for development and secondary

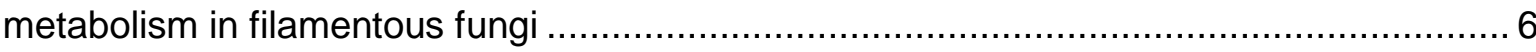

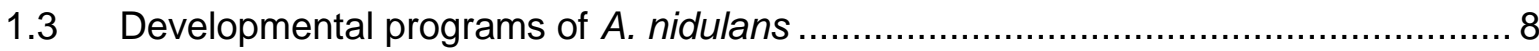

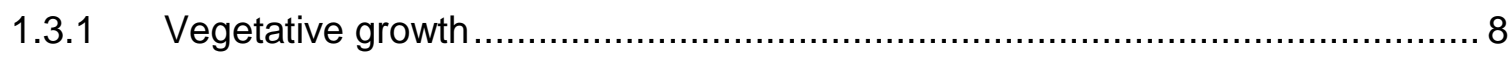

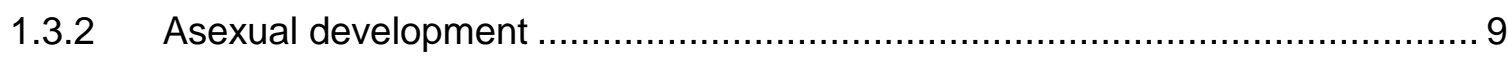

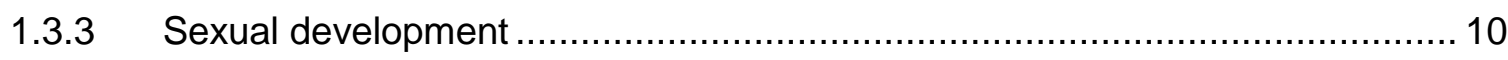

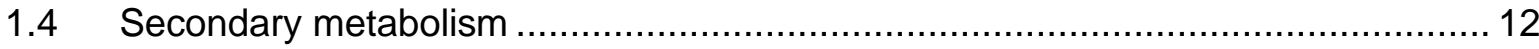

1.4.1 Interconnection of development and secondary metabolite production ........... 12

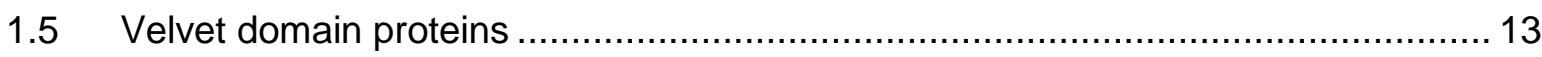

1.5.1 Coordination of development and secondary metabolism by the velvet domain proteins

1.5.2 The multifunctional regulator VelB and selective heterodimer formation of velvet domain proteins in $A$. nidulans.

1.5.3 The fungal-specific velvet domain regulators are structurally similar to NF-kB 18

1.5.4 Domain architecture of velvet proteins ................................................... 20

1.5.5 Intrinsically disordered domains.......................................................... 21

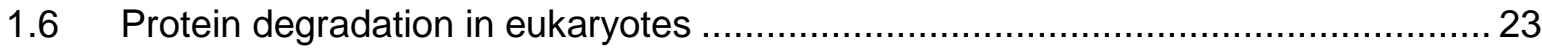

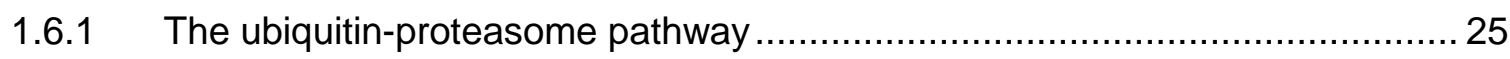

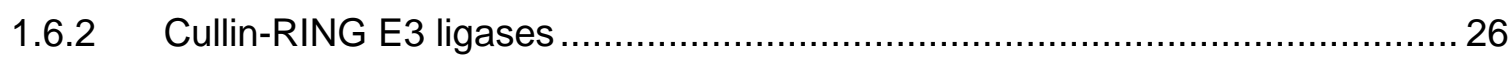

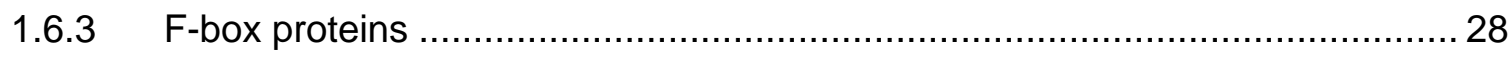

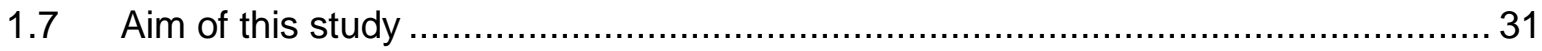

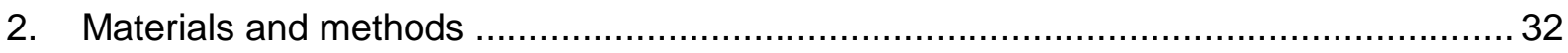

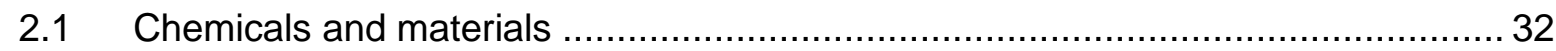

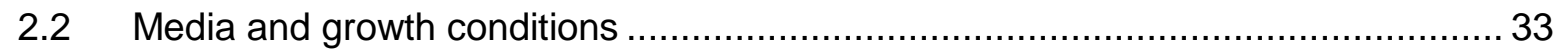

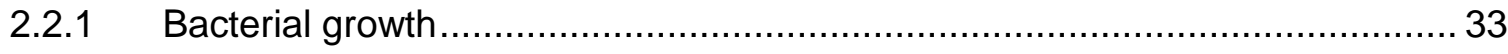

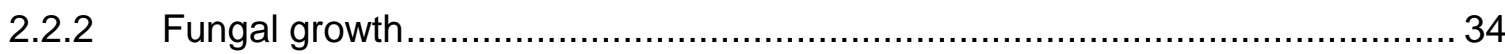




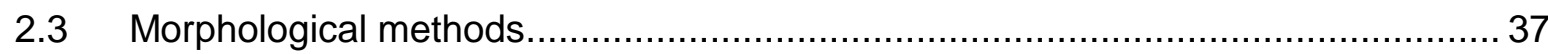

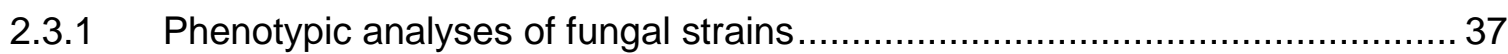

2.3.2 Conidiospore quantification ............................................................. 37

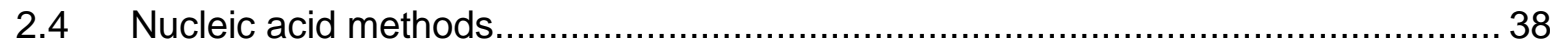

2.4.1 Isolation and purification of plasmid-DNA and linearized DNA fragments ....... 38

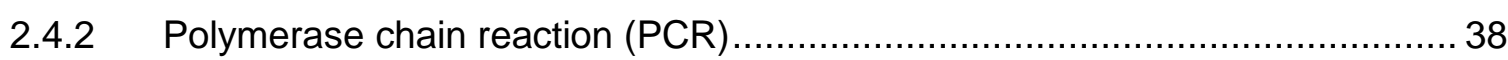

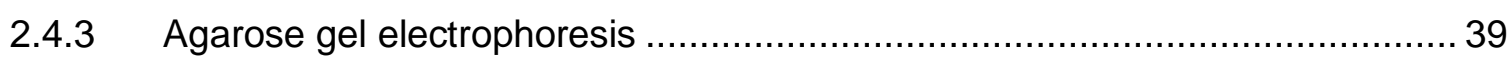

2.4.4 Quantitative real-time polymerase chain reaction ........................................ 39

2.4.5 Isolation and purification of fungal genomic DNA ....................................... 40

2.4.6 Isolation and purification of fungal RNA …................................................ 40

2.5 Plasmid construction for genetic manipulation of $A$. nidulans............................ 41

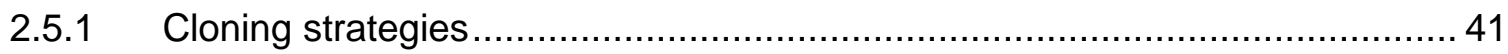

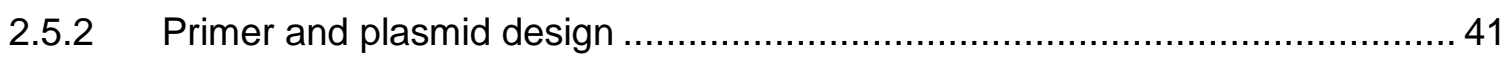

2.5.3 Recyclable marker cassettes as selection markers...................................... 44

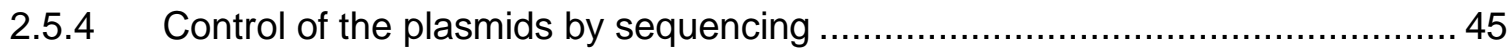

2.5.5 Plasmid and strain construction of $A$. nidulans mutant strains........................ 45

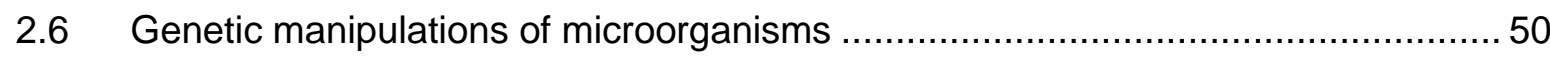

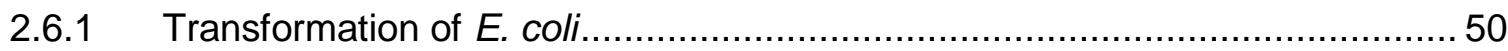

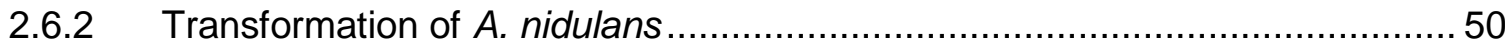

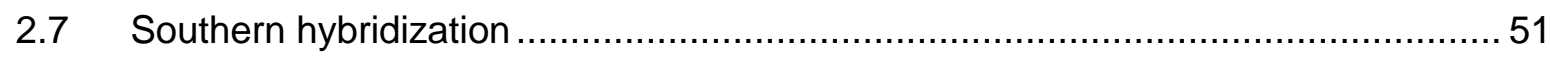

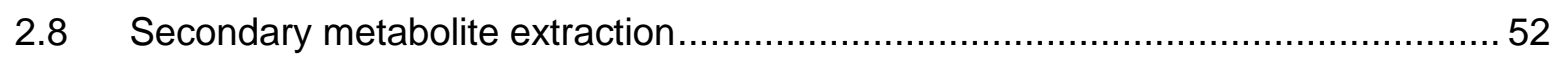

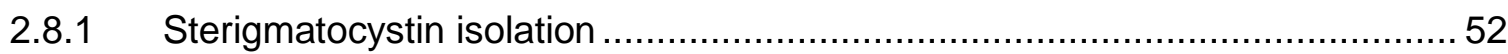

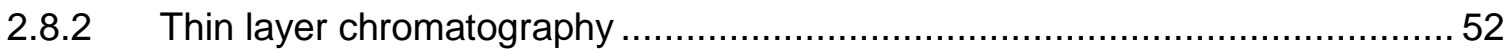

2.8.3 Secondary metabolite extraction for HPLC and LC-MS/MS measurements .... 52

2.8.4 Secondary metabolite analysis by high-performance liquid chromatography (HPLC) coupled with a UV diode array detector (UV-DAD) ...................................... 53

2.8.5 Secondary metabolite analysis by liquid chromatography coupled to tandem

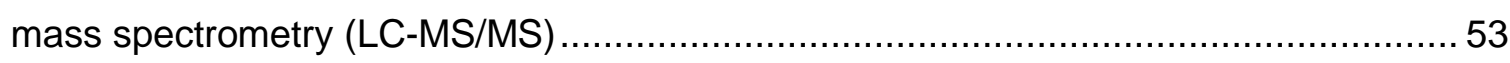

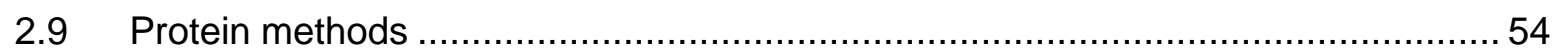

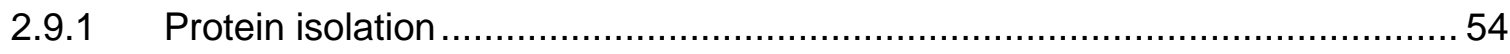

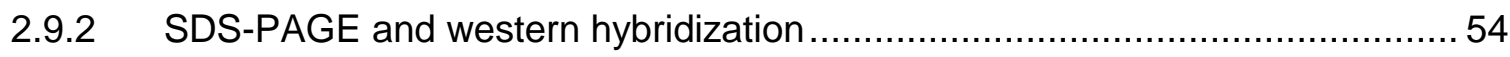

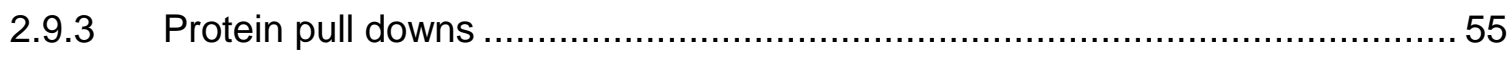

2.9.4 Bioinformatics analyses of protein features......................................... 56

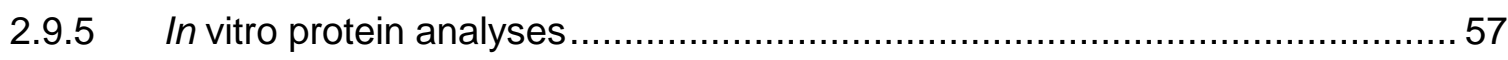


2.10 Identification of proteins form GFP pull downs with mass spectrometry ................58

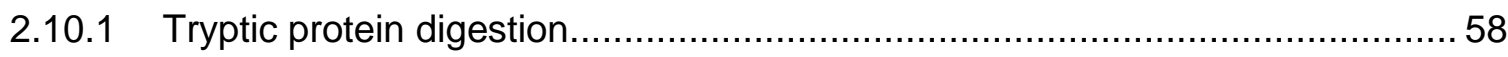

2.10.2 C18 StageTip purification of trypsin-digested samples............................... 59

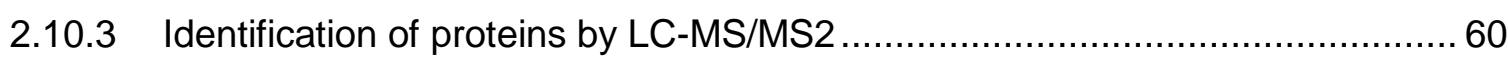

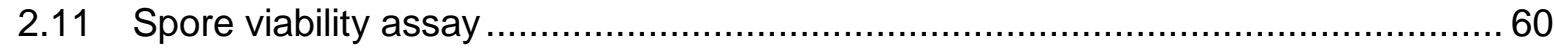

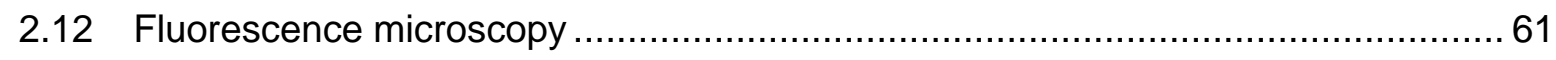

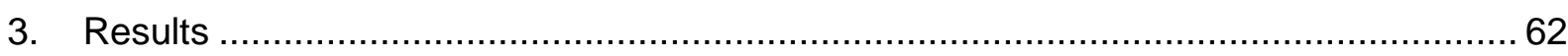

3.1 The intrinsically disordered domain of $A$. nidulans VelB contains an N-terminal motif

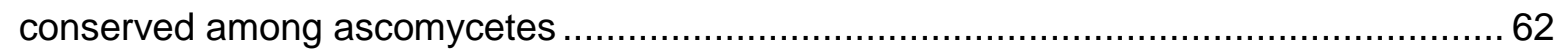

3.2 The VelB intrinsically disordered domain supports selective heterodimer formation

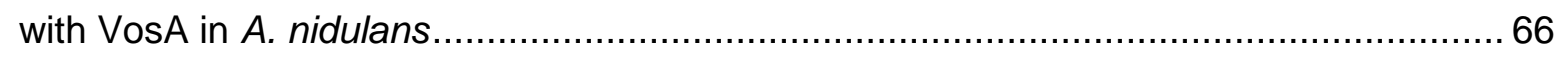

3.2.1 The subcellular localization of VelB is independent of the IDD......................66

3.2.2 VeA-VelB heterodimer formation is independent of the IDD ..........................67

3.2.3 The IDD is required for stable VelB-VosA heterodimer formation .................... 72

3.2.4 The VeIB IDD is required for VelB-VosA heterodimer formation in vivo but not

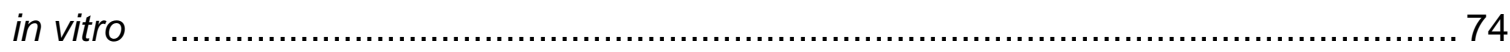

3.2.5 The VeIB IDD is necessary for homodimer formation in vitro ......................... 76

3.3 The IDD of VelB is required for efficient asexual spore formation in A. nidulans .... 78

3.3.1 The survival of conidiospores is independent of the VelB IDD of $A$. nidulans.. 82

3.4 The VelB intrinsically disordered domain is required for the regulation of secondary metabolite production of $A$. nidulans......

3.4.1 The accurate regulation of sterigmatocystin production requires the intrinsically disordered domain of VelB. 83

3.4.2 The VelB-VosA heterodimer regulates austinol and dehydroaustinol biosynthesis 87

3.4.3 The VelB-VeA heterodimer represses the production of orsellinic acid and F$9775 \mathrm{~A}$ and $\mathrm{B}$ 88

3.5 The intrinsically disordered domain of VelB is a possible target for protein degradation 91

3.5.1 The VelB protein is destabilized by the IDD 91

3.5.2 Fbx18 reduces cellular VelB protein levels comprising the intrinsically disordered domain .95

3.6 In silico analyses of the A. nidulans VelB intrinsically disordered domain 101 
3.6.1 The VelB intrinsically disordered domain of $A$. nidulans contains putative Short Linear Motifs

3.6.2 The VelB intrinsically disordered domain might contain targets for phosphorylation

3.7 VelC is an auxiliary regulator of development in $A$. nidulans 106

3.7.1 VelC interacts with VosA in A. nidulans during early sexual development..... 106

3.7.2 Conidiospore formation of $A$. nidulans is delayed in the velB $B^{I D D_{\Delta}} / \mathrm{velC} \Delta$ double mutant

3.7.3 Secondary metabolite production is altered in the $v e / B^{I D D_{\Delta}} / v e / C \Delta$ double mutant 112

3.7.4 The VelC-VosA heterodimer is a repressor of asexual development 115

4. Discussion 119

4.1 Insertion of an intrinsically disordered domain into the VelB velvet domain results in a novel layer of control of the dimerization potential of a fungal transcription factor. 119

4.1.1 A molecular mechanism might enable different VelB complex formations in the nucleus

4.2 The amino acid sequence of the VelB intrinsically disordered domain evolved rapidly in filamentous fungi ..... 124

4.3 Disordered domains play central roles as hubs in protein interaction networks.... 128

4.3.1 VelB as hub in interaction networks. 128

4.3.2 The regulation of the conformational state of intrinsically disordered proteins.....

4.3.3 Putative short linear motifs and phosphorylation sites of the $A$. nidulans VelB IDD

4.4 The VelB protein stability is affected by the intrinsically disordered domain 133

4.4.1 Fbx18 is involved in the regulation of VelB protein levels..... 134

4.5 The VeIB IDD is required for accurate conidiation and secondary metabolite production in $A$. nidulans 138

4.5.1 Dehydroaustinol production requires the VelB-VosA heterodimer 138

4.5.2 High spore densities require the VeIB IDD for conidiospore formation in A. nidulans.

4.5.3 The VelB IDD is required for the accurate regulation of sterigmatocystin production.

4.5.4 Velvet domain protein dimers abolish the production of certain secondary metabolites 


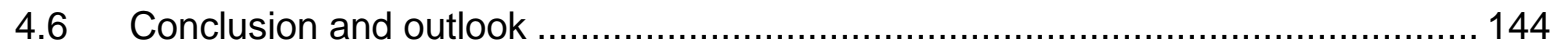

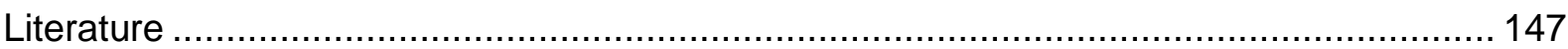

List of abbreviations

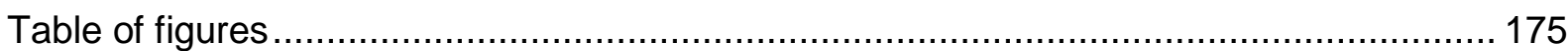

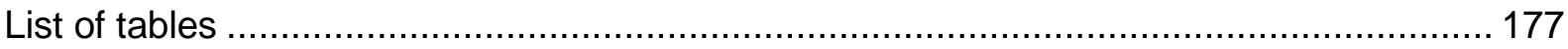

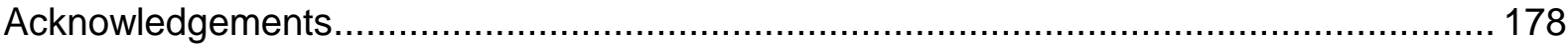

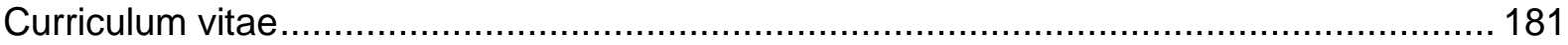




\section{Summary}

The fungal specific velvet domain mediates DNA-binding and protein-protein interaction and is structurally similar to the Rel homology domain of the mammalian immune and infection response NF-KB regulator. Homo- and heterodimers of the four Aspergillus velvet domain proteins VeA, VelB, VelC and VosA control together with methyltransferases as LaeA the expression of developmental and secondary metabolite cluster genes in response to environmental stimuli, such as light or oxygen supply. Only VelB possesses a velvet domain, which is interrupted by an intrinsically disordered domain (IDD). The VelB IDD of Aspergillus nidulans consists of 99 amino acids. The location of this interruption of VelB is conserved in ascomycetes or basidiomycetes with considerable differences in lengths and amino acid sequences. Disordered domains can play central roles as hubs in protein interaction networks. This study focuses on VelB IDD function in the regulation of $A$. nidulans development and secondary metabolism. Deletion of fbox18, encoding a specific F-box protein, resulted in an increased cellular amount of VelB compared to wildtype. F-box proteins are the substrate receptors of E3 SCF ubiquitin ligases. The VeIB IDD also destabilizes the VelB protein and is therefore a possible target for ubiquitin mediated degradation by the $26 \mathrm{~S}$ proteasome. VelB can form a homodimer as well as VelB-VeA or VelB-VosA heterodimers. VosA can form an additional VosA-VelC heterodimer. The molecular mechanisms how the fungal cell controls the appropriate ratios of homo- or heterodimers of the available velvet domain proteins are yet elusive. VelB-VeA and heterotrimeric VelB-VeA-LaeA formation are independent of the presence or absence of the VeIB IDD. Construction of a VelB without IDD revealed that VelB homodimer as well as VelB-VosA heterodimer formation require the IDD of VelB. When only VosA-VosA can be formed and the interaction of VosA with VelB as well as VelC is impaired, the conidiation of the corresponding $v e l B^{I D D_{\Delta}} / v e l C \Delta$ mutant strain is delayed, and increased sterigmatocystin biosynthesis suggests an altered secondary metabolite production. The VelB IDD is required for efficient conidiation and control of secondary metabolism, because VelB without IDD resulted in changes in asexual development and secondary metabolite production. The corresponding vel$B^{I D D}$ deletion mutant strain produced increased aerial hyphae but reduced numbers of conidiospores. Furthermore, it synthesized increased amounts of the mycotoxin sterigmatocystin but decreased levels of austinol and dehydroaustinol compared to wildtype. The results demonstrate that the fungal VelB intrinsically disordered domain is required to allow formation of distinct VelB homo- and heterodimers. This suggests a molecular mechanism where masking or demasking of the IDD could control the ratio of velvet domain protein complexes in response to different environmental stimuli. IDD interacting proteins or IDD posttranslational modifications could change cellular velvet complex ratios and support the appropriate fungal developmental program and secondary metabolism. 


\section{Zusammenfassung}

Die Velvet-Domäne stellt ein DNA-Bindemotif und eine Protein-Protein-Interaktionsdomäne dar, welche strukturelle Gemeinsamkeiten zu der Rel-Homolgie-Domäne von NF-kB, einem Regulator der Immun- und Infektionsreaktion in Säugetieren, besitzt. Homo- und Heterodimere der vier Aspergillus Velvet-Domänen-Proteine VeA, VelB, VelC und VosA kontrollieren zusammen mit Methyltransferasen wie LaeA die Expression entwicklungsspezifischer Gene und Sekundärmetabolit-Gencluster als Antwort auf Umweltreize, wie zum Beispiel Licht und die Verfügbarkeit von Sauerstoff. Nur VelB besitzt eine Velvet-Domäne, die von einer intrinsisch unstrukturierten Domäne (intrinsically disordered domain = IDD) unterbrochen ist. Die VelB IDD von Aspergillus nidulans besteht aus 99 Aminosäuren. Die Position dieser Unterbrechung von VelB ist konserviert in Ascomycota und Basidiomycyota, variiert jedoch erheblich in ihrer Länge und der Sequenz der Aminosäuren. Ungeordnete Domänen spielen eine zentrale Rolle als Netzknoten in Protein-Interaktions-Netzwerken. Die vorliegende Studie konzentriert sich auf die Rolle der VeIB IDD in der Regulation der Entwicklung und des Sekundärmetabolismus in $A$. nidulans. Die Deletion von fbox18, einem spezifischen F-box Protein, führte zu einer erhöhten zellulären Menge von VelB im Vergleich zum Wildtyp. F-box Proteine sind die Substratrezeptoren in E3 SCF Ubiquitin-Ligasen. Die VelB IDD führt auch zu der Destabilisierung des VelB Proteins und ist deswegen ein mögliches Ziel für den Ubiquitin vermittelten Abbau durch das 26S Proteasom. VelB kann Homodimere als auch VelB-VeA oder VelB-VosA Heterodimere bilden. VosA kann außerdem noch VosA-VelC Heterodimere formen. Der molekulare Mechanismus wie die Pilzzelle das Verhältnis der Homo- und Heterodimer der verfügbaren Velvet Proteine kontrolliert ist noch nicht bekannt. Die VelB-VeA Komplexbildung und die Formation des heterotrimerischen Velvet-Komplexes VelB-VeA-LaeA sind unabhängig von dem Vorhandensein oder Fehlen der VeIB IDD. Die Konstruktion von einem VelB ohne die IDD zeigte, dass sowohl die VelB Homodimer, als auch die VelB-VosA Heterodimer-Bildung die VeIB IDD benötigen. Wenn nur VosA-VosA gebildet werden kann und die Interaktion von VosA mit VelB als auch mit VelC behindert ist, ist die Bildung der Konidiosporen in der entsprechenden $v e / B^{I D D_{\Delta}} / v e / C \Delta$ Mutante verzögert und die erhöhte Biosynthese von Sterigmatocystin weist auf einen veränderten Sekundärmetabolismus hin. Die VelB IDD wird für effiziente Konidiosporenbildung und die Kontrolle des Sekundärmetabolismus benötigt, da ein VelB ohne die IDD in veränderter asexueller Entwicklung und Sekundärmetabolismus resultierte. Die entsprechende velB ${ }^{I D D}$ Deletionsmutante produzierte mehr Luftmyzel und weniger Konidiosporen und synthetisierte eine erhöhte Menge des Mykotoxins Sterigmatocystin und weniger Austinol und Dehydroaustinol im Vergleich zum Wildtyp. Diese Ergebnisse zeigen, dass die intrinsisch ungeordnete Domäne von VelB benötigt wird, um die Bildung bestimmter Homo- und 
Heterodimere zu ermöglichen. Das deutet darauf hin, dass ein molekularer Mechanismus existiert, indem die Maskierung und Demaskierung der IDD das Verhältnis der Velvet-ProteinKomplexe als Antwort auf verschiedene Umweltreize kontrollieren kann. IDD interagierende Proteine oder IDD posttranslationale Modifikationen können das zelluläre Velvet-KomplexVerhältnis ändern und das angemessene Pilzentwicklungsprogramm und Sekundärmetabolismus fördern. 


\section{Introduction}

\subsection{Transcription factors}

Multicellular organisms as you find them in many eukaryotes and in some bacteria are much more complex than single cell organisms. They apply the same basic principle of transcriptional regulation by proteins known as transcription factors (TFs). Transcription factors are required for the activation or repression of their specific target genes to adapt and respond to external and internal changes. TFs are DNA-binding proteins, which specifically recognize and bind to nucleotide sequences in promoter regions, cis-regulatory elements, and regulate transcription of corresponding genes (Babu et al., 2004). TFs contain a DNA-binding domain (DBD) for DNA recognition and can additionally contain regulatory domains to respond to a signal from the internal or external cellular environment. TFs are grouped into different classes based on the sequence similarity and hence the tertiary structure of their DBDs (Heinemeyer et al., 1999; Wingender, 1997). The superclasses comprise: basic domain, zinc-coordinating DNA-binding domain, helix-turn-helix, $\beta$-scaffold factors/minor groove contacts and other transcription factors.

Transcription factors are part of transcriptional regulatory networks. These complex networks are necessary for a fast and precise global regulation of target genes, which are essential for cell survival in changing surroundings. Most TFs comprise, beside the DNA-binding domain an oligomerization domain. Most TFs bind DNA as dimers (homo-, heterodimers) or as multimers, which composition also contributes to the DNA-binding specificity. A specific TF might bind to different target sequences in combination with different interaction partners, which thereby enhances its binding capacity (Potoyan et al., 2017).

\subsubsection{Transcriptional regulation of target genes by dimeric complexes}

The formation of oligomers and the diverse combination of transcription factors enables a finetuning of gene expression. Thereby, the limited number of transcription factor coding genes is turned into a larger pool of diverse transcription factor complexes, which can bind to a variety of specific regulatory gene sequences (Potoyan et al., 2017). There are many different examples of transcription factor dimerization and their role in transcriptional regulatory networks.

The Rel homology domain proteins of the NF-kB (nuclear factor kappa-light-chain-enhancer of activated $\underline{B}$ cells) family belong to the Rel homology region class and to the superclass of " $\beta$ Scaffold factors/Minor Groove contacts" transcription factors. They play a central regulatory role in an extensive and complex genetic system and are controlled by a stimulus-responsive signaling system (Potoyan et al., 2017). This transcription factor family is a key regulator of 
immune development, immune response, inflammation and cancer (Mitchell et al., 2016). The mammalian NF-kB transcription factors consist of five monomers (RelA/p65, RelB, c-Rel, NF$\mathrm{kB} 1 / \mathrm{p} 50$, and NF-kB2/p52) and can form 15 possible homo- and heterodimers. This is mediated by the Rel homology domain as dimerization and DNA-binding domain (O'Dea and Hoffmann, 2010). Precise regulation of many target genes is ensured by the different NF-kB dimers and their activity is controlled by $1 \mathrm{kB}$ inhibitor proteins, which bind to and inactivate NF-KB dimers by restricting them to the cytoplasm.

Activator protein 1 (AP-1) transcription factors belong to the leucine zipper factors (bZIP) of the basic domain superclass and are highly evolutionary conserved (Hess, 2004). They are defined as a group of structurally and functionally related members of the Jun and Fos protein family, which have to dimerize before DNA-binding is possible (Wagner, 2001). Gene expression is regulated by different AP-1 dimers in response to a plethora of physiological and pathological stimuli, including cytokines, growth factors, stress signals, bacterial and viral infections and oncogenic stimuli, resulting in the control of a number of cellular processes, such as differentiation and apoptosis (Ameyar et al., 2003; Hess, 2004; Yadav et al., 2017). Recently it was shown, that at different times of hypoxia the AP-1 monomers are expressed in a time-dependent manner to form the appropriate heterodimers and control specific target genes that contribute to cell proliferation and apoptosis (Yadav et al., 2017).

A complex transcriptional network, known as "general amino acid control" in yeast or "crosspathway control" in filamentous fungi coordinates the increased production of a transcriptional activator, homologous to the $H$. sapiens c-Jun protein ATF4, in response to amino acid limitation (Irniger and Braus, 2003). This activator is one of the first bZIP transcription factors described in yeast and filamentous fungi, including Saccharomyces cerevisiae GCN4, Aspergillus CpcA and Neurospora Cpc1, respectively (Hoffmann et al., 2000; Paluh et al., 1988; Paluh and Yanofsky, 1991). These TFs can be functionally exchanged between different fungi, as they bind to the same upstream DNA-binding site like the mammalian c-Jun, thereby increasing the transcription of a plethora of genes involved in amino acid biosynthesis (Hoffmann et al., 2000; Irniger and Braus, 2003; John et al., 1996). This transcriptional activator is composed of a DNA-binding domain and the adjacent leucine zipper as dimerization domain (Harrison, 1991). The leucine zipper domain of yeast GCN4 contains four leucine residues, whereas the transcriptional regulated factor $\mathrm{CpcA}$ of $A$. niger comprises an unusual leucine zipper motif, including only one leucine residue (Wanke et al., 1997). This transcriptional activator is involved in development and virulence in filamentous fungi. The respective $A$. nidulans ortholog CpcA blocks the formation of sexual fruiting bodies in the middle of development during starvation conditions (Hoffmann et al., 2000). CpcA of the fungal pathogen $A$. fumigatus contributes to host infection and the cross-pathway control might act as a general stress response system with a significant role in pulmonary aspergillosis 
(Krappmann et al., 2004). The ortholog of the plant-pathogenic fungus Verticillium longisporum Cpc1 is also required for pathogenicity (Timpner et al., 2013).

The precise timing and tight control of specific target genes is also important for fungi to interconnect developmental programs, pathogenicity and secondary metabolism in response to environmental changes. The heterodimeric homeodomain transcription factor $\mathrm{bE} / \mathrm{bW}$ of the smut fungus Ustilago maydis, encoded by the multiallelic $b$ mating type locus, is sufficient to initiate the switch from non-infectious yeast-like to hyphal growth and to support pathogenic development (Brachmann and Weinzierl, 2001; Heimel et al., 2010; Kämper et al., 1995).

Fungal growth and development is often coordinated with the control of secondary metabolism (Bayram and Braus, 2012; Yu and Keller, 2005). The velvet domain proteins are fungal specific transcription factors, which control the regulation of development with the interconnected secondary metabolism in response to external or internal stimuli (CHAPTER 1.5) (Bayram et al., 2008; Bayram and Braus, 2012). Interestingly, crystal structure analyses revealed structural similarity between the velvet domain and the Rel homology domain of the NF-kB family. Like the NF-KB dimers, the formation and specific ratio of certain velvet domain protein homo- and heterodimers is required for the appropriate regulation of developmental and secondary metabolite gene clusters (Bayram et al., 2008; Calvo et al., 2016; Gao et al., 2017; Park et al., 2012b; Wu et al., 2017). Theses fungal specific TFs were intensively investigated in Aspergillus nidulans, which is a well-studied genetic model organism with a well described sexual and asexual life cycle and intensively studied secondary metabolite production (Braus et al., 2002; Dyer and O'Gorman, 2011; Gerke et al., 2012; Han, 2009).

\subsection{Aspergillus nidulans - a reference organism for development and secondary metabolism in filamentous fungi}

Fungi are ubiquitously distributed on earth and represent the largest group of eukaryotic organisms. The production of secondary metabolites, small, low-molecular-weight bioactive natural products, renders most fungal species either helpful or harmful to humankind (Gerke and Braus, 2014). Aspergilli belong to the phylum of Ascomycota and grow in a wide range of niches. Most representatives of this genus can be found in soils or dead organic matter like composts but there are also parasitic or pathogenic Aspergilli, which colonize plant or animal hosts. The genus Aspergillus includes members of medical, nutritional and industrial importance (de Vries et al., 2017). There are Aspergillus species, which have detrimental effects to humankind, such as $A$. fumigatus and $A$. terreus, causing serious infections in immunocompromised patients and $A$. flavus as a wide-spread crop contaminant (lbarra et al., 2017; Jöhnk et al., 2016; Shlezinger et al., 2017; Slesiona et al., 2012; Yu et al., 2005). Other representatives of this genus are important for many processes in the pharmaceutical, food 
and enzyme industries. Asian cuisine relies on $A$. oryzae for food fermentation and $A$. niger is the main industrial producer of citric acid, which is one of the most widely used food ingredients (Brooke, 1994; A. J. Chen et al., 2016; Despot et al., 2016; Dias et al., 2017; Dodds and Gross, 2007; Wang et al., 2017).

One of the best studied Aspergillus species is A. nidulans. Investigation of this genetic model organism contributed mostly to our knowledge about eukaryotic cell biology and the molecular processes behind. The fungus favors either asexual or sexual development in response to environmental and internal signals like light, temperature, $\mathrm{pH}$, nutrient availability and the oxidative state of the cell. The fungus propagates by forming air-borne conidiophores harboring the mitotic conidiospores during asexual development or reproduces sexually by the formation of fruiting bodies within the soil (FIGURE 1).

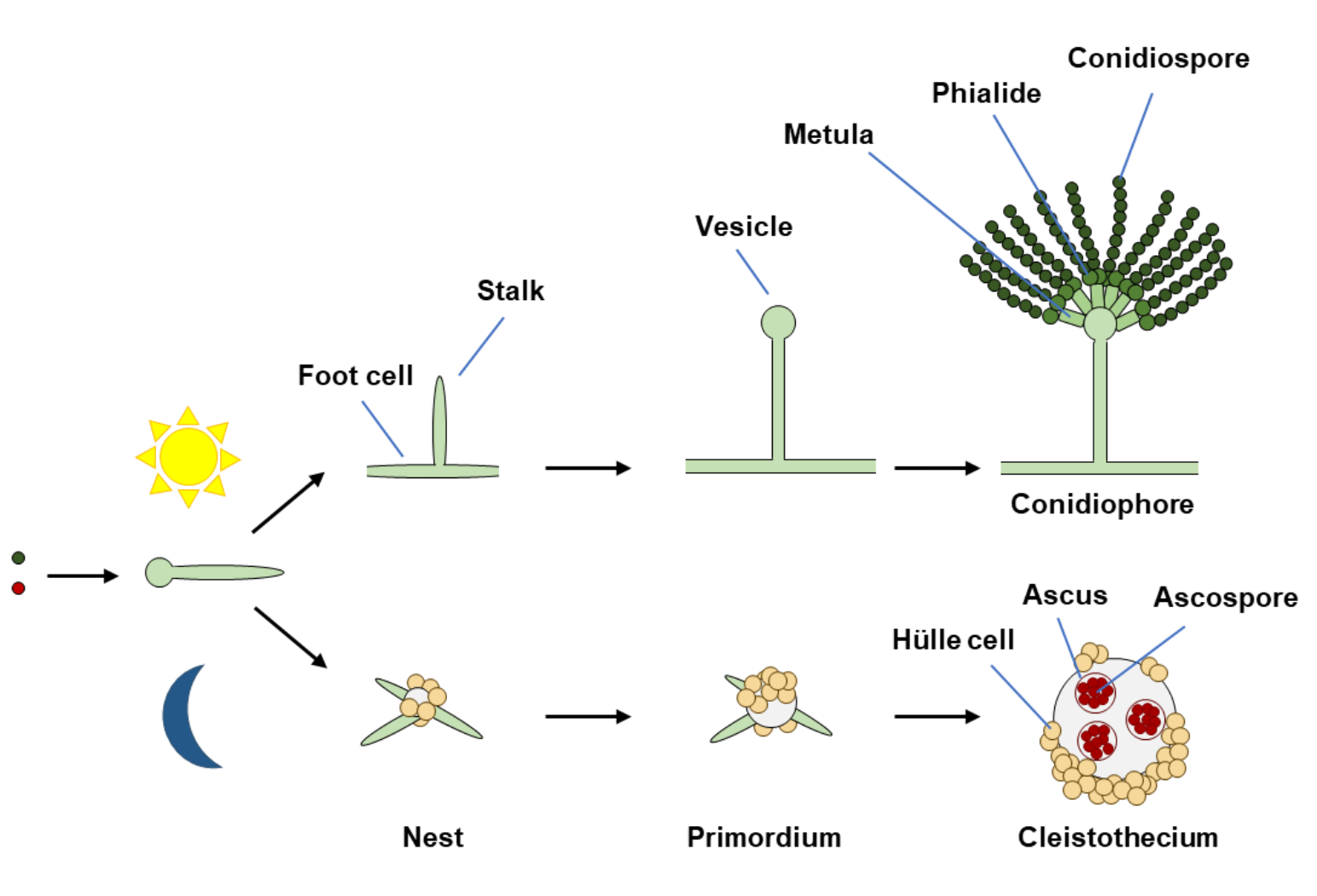

FIGURE 1: Life cycle of $A$. nidulans.

The spore germinates and forms a vegetative hypha. After 18 to 20 hours the hyphae reaches developmental competence and environmental signals, such as light, trigger the induction of asexual or sexual development. In the light asexual development and the production of airborne conidiospores is favored, whereas in the dark under oxygen-limited conditions predominantly sexual cleistothecia are formed (adapted from Bayram and Braus, 2012). 
The genome of $A$. nidulans has a size of $31 \mathrm{Mb}$ distributed among eight chromosomes and is completely sequenced but approximately $90 \%$ of the 10,560 encoded genes remain uncharacterized (Bayram et al., 2016; Cerqueira et al., 2014; Galagan et al., 2005). The short life cycle and the genetic approaches established over the years make $A$. nidulans a favorable eukaryotic model organism. Discoveries made on the molecular level are applicable to its relatives of industrial and medical importance as well as to higher eukaryotic organisms.

\subsection{Developmental programs of $A$. nidulans}

\subsubsection{Vegetative growth}

The life cycle of $A$. nidulans begins with an asexual or sexual spore growing isotropically until a polarity site is established. Germination of the spore is triggered by environmental signals such as light or conidial density (Braga et al., 2015; Herrero-Garcia et al., 2015; Oiartzabal-Arano et al., 2016). Extension of the germ tube leads to the generation of multinucleated and cylindrical cells with a slightly tapered apex, the hyphae (Oiartzabal-Arano et al., 2016). The continuous polarized growth and apical elongation of the vegetative hyphae is characteristic for filamentous fungi (Riquelme, 2013; Takeshita et al., 2014). The constant hyphal extension is achieved by polarized transport of cell wall and plasma membrane material in secretory vesicles to an apical localized structure termed Spitzenkörper (Fajardo-Somera et al., 2015; Schultzhaus et al., 2017). Hyphal branching results in the generation of a complex undifferentiated network of interconnected hyphae called mycelium (Oiartzabal-Arano et al., 2016; Riquelme, 2013; Takeshita et al., 2014). The Spitzenkörper is the organizing center for hyphal growth and represents an accumulation of exocytic and endocytic vesicles. Its position in the hyphal tip determines the growth direction (Fischer et al., 2008; Riquelme, 2013; Schultzhaus et al., 2017). Fungi use these exocytic vesicles to carry and secrete a multitude of components to react to and communicate with their environment. The opposite of exocytosis, endocytosis, is required as well for communication. Endocytosis enables that external signals are not just send but also received by the fungal cell. Furthermore, endocytic vesicles serve to remove cell wall-synthesizing enzymes from the tip, which are embedded in the plasma membrane (Caballero-Lima et al., 2013; Schultzhaus et al., 2015). The Spitzenkörper represents a signaling hub, which senses and adapts to environmental changes and in this context, is also important for protein-recycling at the hyphal tip (Harris, 2009). After 18 to 20 hours hyphal cells reach developmental competence (Axelrod et al., 1973). In the competence state the cells become susceptible for external stimuli such as light, which results in the induction of either asexual or sexual development (Adams et al., 1998; Axelrod et al., 1973; Bayram and Braus, 2012; Pöggeler et al., 2006). 


\subsubsection{Asexual development}

In an illuminated environment under the availability of oxygen $A$. nidulans competent cells favor asexual development (Adams et al., 1998). Light and other external stimuli, such as temperature, $\mathrm{pH}$ and nutrient supply as well as internal signals, like metabolites, trigger the induction of asexual development (Oiartzabal-Arano et al., 2016; Rodríguez-Urra et al., 2012). The result is the production of asexual spore-harboring conidiophores. Conidiophore development starts with the formation of a thick-walled foot cell, which forms an aseptic stalk that terminates in a multinucleated vesicle (FIGURE 2) (Axelrod et al., 1973; Mims et al., 1988; Park and Yu, 2012; Yager, 1992). Budding of the vesicle produces a layer of uninucleated metulae, which build two to three spore-bearing phialides per metula (Mims et al., 1988). Ultimately, repeated mitotic divisions of the phialides result in the formation of haploid air-borne conidiospores (FIGURE 2) (Adams et al., 1998; Garzia et al., 2013). The whole process of conidia production takes approximately 15 to 24 hours post induction of asexual development (Fischer, 2002; Ruger-Herreros et al., 2011; Yu et al., 2010).

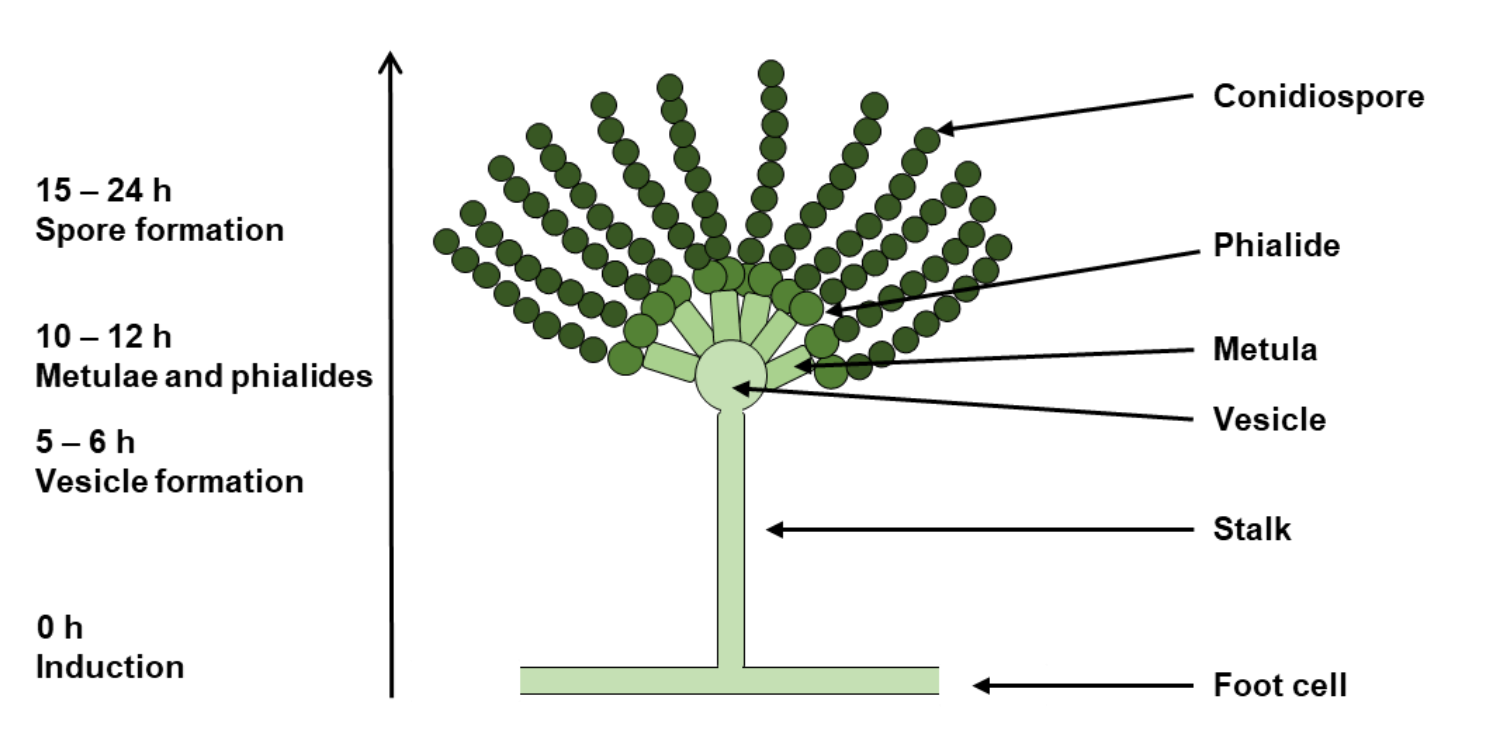

FIGURE 2: The conidiophore of $\boldsymbol{A}$. nidulans.

Conidiophore formation over time is depicted. First a thick-walled stalk is formed, which terminates in a multinucleated vesicle. Budding processes result in the formation of metulae, which themselves build two or more phialides. After 15 to 24 hours conidiophore formation results in the production of the air-borne conidiospores by repeated mitotic divisions of the phialides (adapted from Yu, 2010).

Conidiation is genetically controlled by the central developmental pathway (CDP) including the $\mathrm{C} 2 \mathrm{H} 2$ zinc finger transcription factor $\underline{B}$ ristle $\underline{A}(\mathrm{BrlA})$ as the master regulator (Adams et al., 1998; Ruger-Herreros et al., 2011). Induction of brlA gene expression is the key step in this pathway, which is required and sufficient to activate conidiation (Adams et al., 1988). BrlA activates the expression of abacus $\underline{A}(a b a A)$ in the mid-phase of conidiospore formation. AbaA 
is necessary for phialide differentiation (Adams et al., 1988, 1990; Andrianopoulos and Timberlake, 1993; Boylan et al., 1987; Sewall et al., 1990). AbaA thereby activates wet-white $\underline{A}$ (wetA) expression, which gene product is required for the stability of mature conidia and for the biosynthesis of conidiospore wall components (Lee et al., 2016).

The CDP is activated by FluG (luffy $\underline{\mathrm{G}}$ ) and products of the flb (luffy with low brlA expression) genes, which are part of the upstream developmental activators (UDA) (Adams et al., 1988, 1998; Wieser et al., 1994). The repression of brlA prevents premature asexual development during vegetative growth, which is achieved by SfgA (suppressor of $\underline{\text { fluG }} \underline{A}$ ) and by VosA

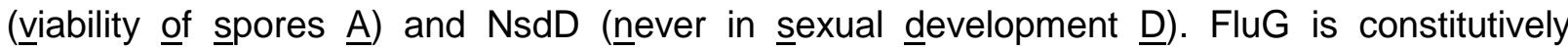
produced and is needed to synthesize a low-molecular-weight extracellular signal molecule to initiate conidiophore formation (CHAPTER 1.4.1) (Lee and Adams, 1994; Lee et al., 2014; Seo et al., 2003, 2006). When FluG reached a certain threshold it removes the repressive effects of SfgA, which is followed by dissociation of VosA and NsdD from the brlA promoter (Lee et al., 2014; $\mathrm{Ni}$ and $\mathrm{Yu}, 2007$ ). Subsequently, the Flb factors can bind to the brlA promoter and initiate asexual development (Garzia et al., 2010). This whole process occurs in a time dependent manner.

\subsubsection{Sexual development}

Many environmental and internal factors besides the absence of light and increased carbon dioxide concentrations like the availability of nutrients, surface contact, pheromones or the redox status of the cell promote sexual development, resulting in the production of closed sexual fruiting bodies, the cleistothecia (Busch and Braus, 2007; Ugalde and Rodriguez-Urra, 2016). They are the overwintering structures and contain the sexual meiospores, the ascospores (Braus et al., 2002). The sexual cycle of $A$. nidulans starts with the fusion of two haploid ascogonial hyphae to a dikaryon. $A$. nidulans is a homothallic fungus, meaning that it is self-fertile and able to develop sexually in the absence of a mating partner. This process, called selfing, results in offspring genetically identical to the parent. $A$. nidulans cells are also able to undergo sexual development in combination with partner with different genetic equipment. This process, termed mating, is necessary for genetic recombination to occur (Braus et al., 2002; Busch and Braus, 2007). Meiotic division diminishes the genome to a haploid after karyogamy. Subsequently, ascosporogenesis, the enclosure of the haploid nuclei in newly formed cells, yields the ascospores (FIGURE 1). Aspergilli as part of the ascomycetes are characterized by the formation of the name-giving structure: the ascus. The ascus is a sac-like closed structure, which encompasses the ascospores inside the fruiting body (FIGURE 1) (Braus et al., 2002; Pöggeler et al., 2006). The visible fruiting body is built by the ascocarp, protecting the asci (Greek: askos = sac; karpos = fruit). In A. nidulans the ascocarp is called cleistothecium (Greek: kleistos = closed; theke = case), which can harbor up to 
80,000 ascospores (Braus et al., 2002). The Hülle cells differentiate from hyphae and form nest-like structures around the developing cleistothecium (Latin: nidulans = nest) and are proposed to nourish and protect the maturing fruiting body (Braus et al., 2002; SarikayaBayram et al., 2010). Tissue specific gene products, as laccase II are synthesized in the Hülle cells, which can be transferred to the immature fruiting body, called primordium (Hermann et al., 1983; Scherer and Fischer, 1998). Whereas the air-borne asexual conidiospores are easily distributed by the wind, sexual ascospores are released from the mature cleistothecium and are readily transported by water (Busch and Braus, 2007; Pöggeler et al., 2006).

The intracellular transduction of extra- and intracellular signals resulting in induction of sexual development requires various factors. Amongst these are G-proteins, such as FadA (Fluffy autolytic dominant $\underline{A}$; Ga-subunit), SfaD (Suppressor of $\underline{F}$ lb $\underline{A} \underline{D}$; G $\beta$-subunit) and GpgA (G-protein gamma $\underline{A} ; G_{y}$-subunit) and the mitogen-activated protein kinase SakA/HogA (Busch and Braus, 2007; Pöggeler et al., 2006). Furthermore, several transcription factors, such as SteA (Sterile12-like $\underline{A}$ ), NsdD ( (Dopex $\underline{A}$ ), MedA (Medusa $\underline{A}$ ) and CpcA ( ross pathway control $\underline{A}$ ) are associated with the regulation of the formation of cleistothecia (Busch and Braus, 2007). One fifth of the genome is differentially expressed in A. nidulans during fungal differentiation (Bayram et al., 2016). Light response occurs quickly in $A$. nidulans, whereas the absence of light results in a 24 hour delay in gene expression. At late sexual development (after 72 hours of growth under sexual development inducing conditions) expression of 1168 genes (11\% of the genome) is upregulated (Bayram et al., 2016). This delay is presumably required for initiation and reprogramming of the differentiation process of the fungal cell, finally resulting in the production of sexual fruiting bodies. The expression of genes required for the biosynthesis of certain hormone-like psi-factors (precocious sexual inducer), which derived from lipids, is already induced in vegetative growth. Psi-factors determine the timing and correct execution of asexual or sexual development (Tsitsigiannis et al., 2005). Specific psi-factors are enriched at the beginning of sexual development, which presumably drives the sexual differentiation process (Bayram et al., 2016)

In addition to the regulation at the transcriptional level, posttranslational control and degradation of the cellular proteins contribute to successful fruiting body formation. The ubiquitin-dependent proteasomal protein degradation machinery is negatively regulated by the COP9 signalosome (CSN). Its accurate function is required for the maturation process of cleistothecia and a defective CSN results in the blockade of sexual development at the primordial stage (Beckmann et al., 2015; Busch and Braus, 2007; Draht et al., 2007; Meister et al., 2016). 


\subsection{Secondary metabolism}

During the asexual or sexual development $A$. nidulans produces a variety of secondary metabolites. They are described as low-molecular weight bioactive natural substances, which are produced by plants and many microorganisms, including bacteria and fungi (Bayram and Braus, 2012; Brakhage, 2013). Secondary metabolites, in contrast to primary metabolites, are dispensable for normal growth and survival of the organism. Nevertheless, they play a role in cellular processes including transcription, development and communication between one species or different species for symbiotic interactions or to defend themselves against rivals (Brakhage, 2013; Macheleidt et al., 2016). The biological activities of secondary metabolites can be beneficial or harmful to humankind.

A. nidulans is known to produce various secondary metabolites, like penicillins, sterigmatocystin, benzaldehydes (e.g. DHMBA), emericellamides, orsellinic acid, orcinol and orcinol derivatives, diindols (e.g. DHPDI), austinol and dehydroaustinol. (Brakhage, 2013; Gerke et al., 2012; Gerke and Braus, 2014; Lo et al., 2012; Nahlik et al., 2010). Fungal gene clusters, encoding secondary metabolite biosynthetic enzymes, are silent under standard cultivation conditions and fungi were underrated of the amounts of secondary metabolites they can produce (Gerke and Braus, 2014). The reservoir of so far uncharacterized natural compounds is rich in filamentous fungi, especially in Aspergilli (Alberti et al., 2017; Brakhage, 2013). The ors gene cluster, required for the biosynthesis of orsellinic acid and the cathepsin $K$ inhibitors F-9775A and F-9775B, was the first cryptic gene cluster which was characterized in A. nidulans (Bok et al., 2009; Schroeckh et al., 2009). Co-cultivation of $A$. nidulans with Streptomyces hygroscopicus led to the discovery of orsellinic acid, F-9775A and B, which initiation of production depends on the direct contact between the fungus and the bacterium (Schroeckh et al., 2009). The production of these compounds upon physical contact could be a putative defense mechanism of $A$. nidulans against the bacterium $S$. hygroscopicus. Furthermore, cultivation of $A$. nidulans under specific culture conditions or the deletion of certain genes, such as veA, mvIA or csnE results in the production of orsellinic acid and F-9775A and B (Bok et al., 2013; Nahlik et al., 2010; Sanchez et al., 2010). This shows that secondary metabolites, which are not detectable under laboratory conditions, are often produced as response to their environment, including other species or changing surroundings.

\subsubsection{Interconnection of development and secondary metabolite production}

Developmental programs can be induced by certain secondary metabolites. The fluG gene of A. nidulans was initially proposed to be required for the early induction of conidiation by an extracellular signal, which is not produced by fluG defective mutants (Lee and Adams, 1994). fluffy mutants are non-sporulating mutants, which only form undifferentiated aerial hyphae 
(Rodríguez-Urra et al., 2012). Further investigations revealed that the supplementation of wildtype extracted dehydroaustinol and diorcinol restored the conidial phenotype in a fluG deletion mutant (Rodríguez-Urra et al., 2012). It was examined that the meroterpenoid dehydroaustinol forms an adduct with diorcinol, preventing the formation of dehydroaustinol crystals and therefore enabling the activation of conidiation at the aerial hypha surface (Rodríguez-Urra et al., 2012; Ugalde and Rodriguez-Urra, 2016).

Cluster-specific transcription factors often control the expression of secondary metabolite gene clusters and master regulators of secondary metabolism interconnect secondary metabolite production with developmental programs (Bok and Keller, 2004; Calvo et al., 2002). Like development, activation of secondary metabolite biosynthesis is therefore triggered by environmental signals, such as light, temperature, $\mathrm{pH}$, the availability of nutrients and the presence of organisms (Bayram et al., 2008; Brakhage, 2013). The velvet domain family proteins are bridging factors between development and secondary metabolism (CHAPTER 1.5). $\underline{V}$ elvet $\underline{A}(\mathrm{VeA})$ contributes to the regulation of developmental programs and secondary metabolite production in response to environmental signals. Secondary metabolism is changed and sterigmatocystin production abolished in the veA deletion mutant. VeA controls afIR gene expression, which is the ortholog of the A. flavus and $A$. parasiticus aflatoxin regulator afIR and is the major regulator of sterigmatocystin and penicillin biosynthesis in $A$. nidulans (Kato et al., 2003). Sterigmatocystin is a potent mycotoxin and in several Aspergilli it is the penultimate precursor of aflatoxin. Aflatoxin is a well-known global carcinogenic, with hepatotoxic, genotoxic and immunotoxic effects in multiple species and is strongly linked to the development of hepatocellular carcinoma, liver tumors (J. Chen et al., 2016; Mughal et al., 2016). Sterigmatocystin is related to apoptosis and the disproportion between reactive oxygen species (ROS) and the ROS protection systems in pathogen invaded host cells (J. Chen et al., 2016).

Secondary metabolite regulators are represented by SirtuinA (SirA), which represses the production of sterigmatocystin, austinol and dehydroaustinol. The multicluster regulator $\underline{A}$ (McrA) or the remediator of secondary metabolism $\underline{A}(\mathrm{RsmA})$ also regulate a number of secondary metabolite genes (Itoh et al., 2017; Oakley et al., 2017; Yin et al., 2013).

\subsection{Velvet domain proteins}

\subsubsection{Coordination of development and secondary metabolism by the velvet domain proteins}

The velvet domain proteins represent a class of fungal-specific transcription factors and interconnect development and secondary metabolism in filamentous fungi (Bayram et al., 2008; Bayram and Braus, 2012). The velvet domain regulators are best studied in 
ascomycetes and conserved from basidiomycetes to chytridiomycetes, but absent in single cell yeasts like Saccharomyces cerevisiae or Candida albicans. These yeasts also lack secondary metabolite gene clusters (Bayram and Braus, 2012; Gerke and Braus, 2014; Ni and Yu, 2007). The velvet domain protein family of $A$. nidulans comprises four proteins: $\underline{V}$ elvet $\underline{A}(\mathrm{Ve} A)$, Velvet-like $\underline{B}$ and $\underline{C}($ VelB/C) and VosA.

VeA was discovered more than half a century ago in a random mutagenesis screen in A. nidulans (Käfer, 1965). These mutagenesis experiments produced a strain with a point mutation in the start codon of the veA gene (ATG to ATT), which led to a shift in the start of mRNA translation at the next possible initiation codon. This shift in translation initiation resulted in a gene product that misses the first 36 amino acids. The respective mutant gene was denominated as veA1 (Käfer, 1965; Kim et al., 2002; Mooney et al., 1990; Yager, 1992). Conidiation is independent of light in the veA1 mutant strain, in contrast to the wild type (so called $\left.v e A^{+}\right)$. This characteristic rendered the mutant an attractive background strain in the laboratories, as it produces high numbers of conidiospores during growth in incubators in the dark without the necessity for illumination. The veA1 mutant, besides the increased conidiation, exhibits a reduced and delayed fruiting body formation independent of illumination (Calvo et al., 2016; Yager, 1992). Further analysis of the veA gene, its regulatory mechanism and characterization of the gene product followed several years later, because the protein exhibited at this time no known functional domain. The fact that the veA1 strain produces conidiospores independently of illumination and the finding that the fungus with a $v e A^{+}$genetic background needs red light to develop asexually led to the hypothesis that the VeA protein is crucial for red light-induced conidiation (Mooney and Yager, 1990). Characterization of the fungal red phytochrome-like protein FphA in $A$. nidulans interacting with $\mathrm{VeA}$ in the nucleus supports this hypothesis (Blumenstein et al., 2005; Purschwitz et al., 2008). Kim and colleagues further described the regulatory role of $\mathrm{VeA}$ in 2002. Deletion of the veA gene results in a mutant strain not being able to produce cleistothecia, whereas the $v e A^{+}$ overexpression mutant produced an increased number of sexual fruiting bodies (Kim et al., 2002). The veA gene product acts as a negative regulator of asexual development and as a positive regulator of sexual development (Kim et al., 2002; Kurtz and Champe, 1981; Yager, 1992). Besides its regulatory role in development, $v e A$ functions as master regulator of secondary metabolism in $A$. nidulans. The production of penicillin is decreased and sterigmatocystin production abolished (CHAPTER 1.4.1) in the absence of veA. Expression of the isopenicillin synthetase $\underline{A}$ gene ipnA is increased in the deletion strain. In contrast, expression of the key gene acvA involved in the first step of penicillin synthesis, forming the tripeptide $\delta$-(L-a-aminoadipyl)-L-çysteinyl-D-valine (ACV), is decreased (Brakhage et al., 1992). In conclusion, $v e A$ is necessary for both sterigmatocystin and penicillin production (Calvo et al., 2016; Kato et al., 2003). 


\subsubsection{The multifunctional regulator VelB and selective heterodimer formation of velvet domain proteins in $A$. nidulans}

VelB is important for both, asexual and sexual development, depending on the spatially and timely dimer formation with other velvet domain proteins. VelB acts as light-dependent multifunctional regulator of fungal development and secondary metabolism in $A$. nidulans (Bayram et al., 2008; Park et al., 2012b). Besides regulation of developmental programs, it is required for sterigmatocystin production, spore viability and trehalose biogenesis (Bayram et al., 2008; Park et al., 2012b). Deletion of velB results in reduced and delayed conidiation and abolished sexual development whereas the amount of conidiospores is increased and sexual development is not affected in the overexpression mutant (Park et al., 2012b). VelB was shown to positively influence the central regulatory pathway $b r l A \rightarrow a b a A \rightarrow$ wet $A$, which is necessary for the proper progression of conidiation (CHAPTER 1.3.2) (Adams et al., 1998; Park and $\mathrm{Yu}, 2012)$. VelB is required but only conditionally sufficient to activate conidiation as the overexpression of $v e l B$ is not sufficient to induce the activation of the key asexual regulator gene brlA but shows an increased production of asexual conidiospores. Its expression is positively affected by AbaA, which directly binds to the velB promoter (Park et al., 2012b). VelB does not contain a nuclear localization signal (NLS) and its nuclear entry is enhanced by VeA during fungal growth, which itself binds to the a-importin KapA (FIGURE 3) (Bayram et al., 2008). Migration of the VelB-VeA-KapA complex into the nucleus is regulated by different factors, such as light or the LaeA like methyltransferase $\underline{E}(\mathrm{LImF})$. The entry is also controlled by a heterodimer formed by the two methyltransferases, VipC ( $\underline{\operatorname{VeA}}$ interacting protein $\underline{\mathrm{C}}$ ) and VapB (ㅁipC associated protein B) (Blumenstein et al., 2005; Palmer et al., 2013; Purschwitz et al., 2008). The VipC-VapB dimer is attached to the membrane through the FYVE zinc finger protein VapA ( $\underline{\text { VipC }}$ associated protein $\underline{\mathrm{A}}$ ) in the cytoplasm and is released from VapA after reception of an unknown signal (FIGURE 3) (Sarikaya-Bayram et al., 2014, 2015). Once the VelB-VeA-KapA complex enters the nucleus, KapA dissociates from the complex and the VelB-VeA heterodimer activates sexual development and coordinates secondary metabolism through the interaction with different methyltransferases (see CHAPTER 1.4.4) (FIGURE 3) (Bayram et al., 2008; Palmer et al., 2013; Sarikaya-Bayram et al., 2010, 2014). A competition for VelB to form the VelB-VosA heterodimer or the heterotrimeric VelB-VeA-LaeA velvet complex in the nucleus is assumed. As VeA enhances nuclear entry of VelB, an exchange of VeA for VosA or another VelB in the nucleus is suggested (Sarikaya-Bayram et al., 2010). 


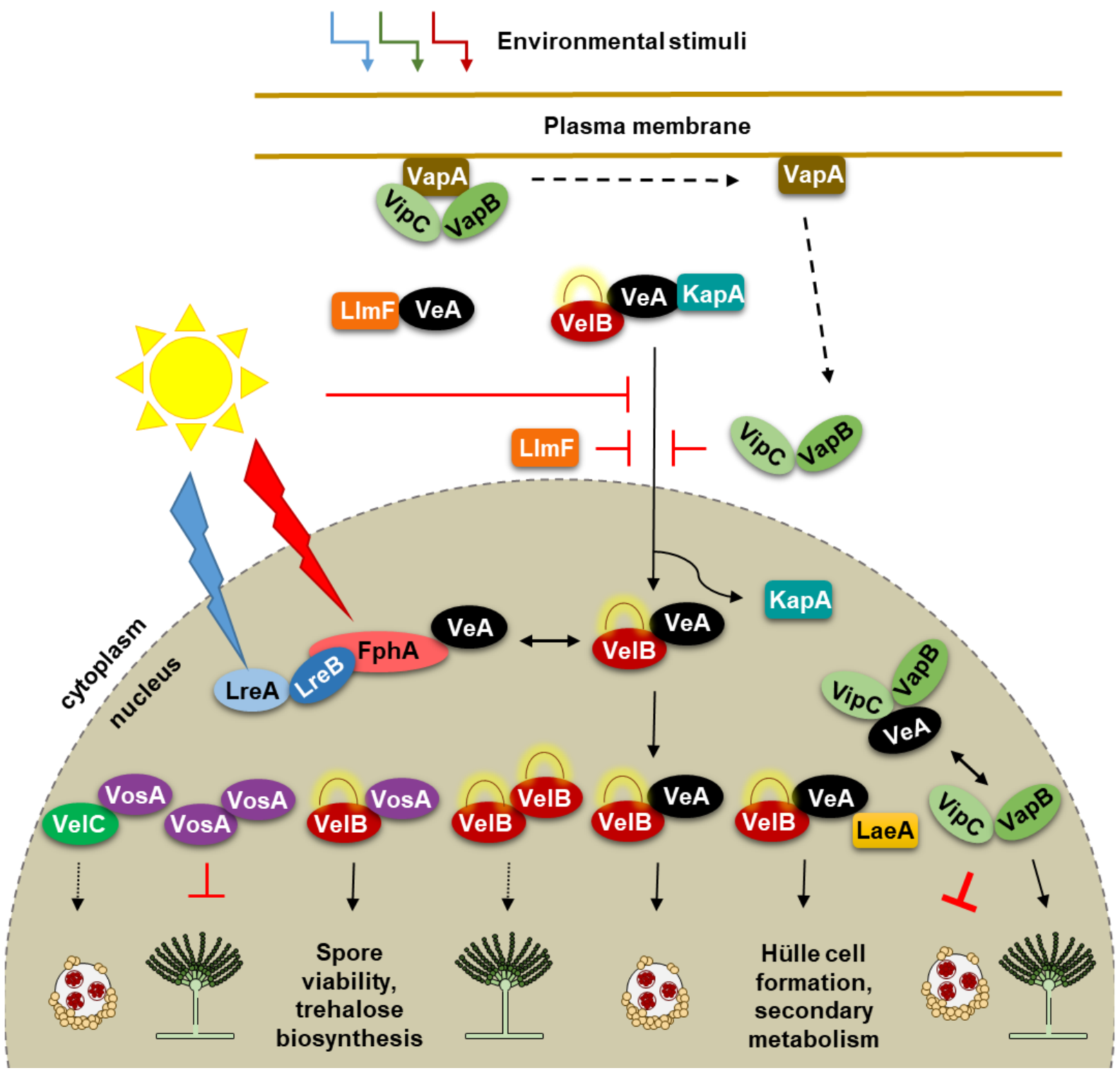

FIGURE 3: The regulatory network of $A$. nidulans velvet proteins.

The a-importin KapA shuttles the VelB-VeA heterodimer into the nucleus. The nuclear entry is suppressed by light as well as by the LaeA-like methyltransferase $\underline{F}$ LImF and the VipC-VapB methyltransferase heterodimer. The VipC-VapB complex is released from the membranebound zinc finger protein VapA upon the reception of an unknown signal (e.g. light, $\mathrm{pH}, \mathrm{CO}_{2}$, $\mathrm{O}_{2}$, starvation). KapA dissociates from the VelB-VeA heterodimer in the nucleus and both velvet domain proteins can form complexes with other proteins. VeA interacts with the phytochrome FphA, the red light receptor. Together with the blue light receptors LreA-LreB the tetrameric VeA-FphA-LreB-LreA complex is formed. VelB-VeA forms together with the methyltransferase LaeA the heterotrimeric velvet complex. VelB-VeA or VelB-VeA-LaeA activates sexual developmental genes as well as secondary metabolite gene clusters. VeA might bind to the VipC-VapB heterodimer and either VeA-VipC-VapB or VipC-VapB activates asexual and represses sexual development. Apart from the VelB-VeA complex, VelB also forms homodimers, which presumably positively influences asexual development. The VelBVosA heterodimer probably binds to target sequences of trehalose biosynthetic genes, required for the viability of spores. The VosA-VosA homodimer serves as repressor of asexual genes. Furthermore, VosA might interact with VelC, which presumably positively influences sexual development (adapted from Sarikaya-Bayram et al., 2015). The yellow loop attached to VelB depicts the intrinsically disordered domain present in the velvet domain of VelB. 
The decision for the right interaction partner is assumed to be time- and cell-type specific, whereas the different complexes can also be built at the same time (Bayram et al., 2008; Gerke and Braus, 2014). The VelB-VosA heterodimer is important for the control of conidial germination, trehalose biogenesis and spore viability ( $\mathrm{Ni}$ and $\mathrm{Yu}, 2007$ ).

VosA is a negative regulator of asexual development, which binds to and represses expression of $\mathrm{brlA}$, which encodes the master regulator of conidiation (Ahmed et al., 2013; Ni and $\mathrm{Yu}$, 2007). Furthermore, VosA is also important for spore viability and trehalose biogenesis in $A$. nidulans ( $\mathrm{Ni}$ and $\mathrm{Yu}, 2007$ ). Like velB, vosA expression is also activated by $A b a \mathrm{~A}$ in vesicles and phialides. The deletion of $v e l B$ or $\operatorname{vos} A$ results in decreased viability of spores in $A$. nidulans ( $\mathrm{Ni}$ and $\mathrm{Yu}, 2007$; Sarikaya-Bayram et al., 2010). Additionally, the vosA deletion mutant exhibits conidiophore formation in submerged cultures and VosA, probably as the VosA-VosA homodimer, represses brlA expression (Ahmed et al., 2013; Ni and Yu, 2007). Genetic data imply interdependence of VelB and VosA regarding the execution of trehalose biogenesis, spore maturation and long-term viability. The VelB-VosA heterodimer presumably is required for regulation of these processes and for the control of precocious formation of conidiospore germination in a negative manner (Ahmed et al., 2013; Ni and Yu, 2007; Park et al., 2012b; Sarikaya-Bayram et al., 2010).

The $\beta$-glucan synthase gene $f k s A$ and the $\underline{V}$ osA/VelB-activated developmental gene $\underline{A}$ ( vadA) are presumably targets of the VelB-VosA heterodimer (Park et al., 2015, 2017). VelB and VosA were shown to bind to the $f k s A$ promoter region in conidiospores and VosA also in ascospores, thereby controlling the correct amount of $\beta$-glucan in asexual and sexual spores (Park et al., 2015). The proper regulation of sporulation and sporogenesis genes, $b r l A, f k s A$, $\operatorname{tps} A$ and $\operatorname{or} A$, requires $\mathrm{Vad} A$, which is supposed to be a regulator of development and secondary metabolism (Park et al., 2017). In other filamentous fungi VelB and VosA orthologs are also involved in spore maturation and development and secondary metabolism. For example, similar to the defects of $A$. nidulans deletion strains, the deletion of $\operatorname{vos} A$ and velB in A. fumigatus results in a $50 \%$ decrease of conidial trehalose, reduced conidiospore viability and increased sensitivity towards oxidative and UV stresses (Park et al., 2012a; SarikayaBayram et al., 2010). The respective VosA and VelB orthologs of the human fungal pathogen Histoplasma capsulatum, Ryp2 and Ryp3 are required for pathogenic yeast-phase growth and are essential for the production of viable spores and the regulation of conidiospore production at room temperature (Webster and Sil, 2008).

In the heterothallic maize pathogen Cochliobolus heterotrophus the VelB and VosA orthologs, namely Vel2 and Vos1, play essential roles in sexual and asexual development (Wang et al., 2014). Deletion mutants are also more sensitive towards oxidative and thermal stresses and trehalose amounts are decreased. Furthermore, Vel2 and Vos1 control asexual spore 
production in a negative manner, whereas they positively regulate the spore morphology (Wang et al., 2014).

VelB orthologs, besides being required for development, were shown to be involved in virulence of pathogenic fungi. The VelB ortholog Vel2 of Fusarium fujikuroi is required for the coordination of sporulation and secondary metabolism and affects virulence of this pathogenic fungus on rice (Wiemann et al., 2010). The velvet domain proteins of pathogenic ascomycetes Valsa mali and Curvularia lunata were recently analyzed regarding their involvement in pathogenicity (Gao et al., 2017; Wu et al., 2017). It was suggested that VmVelB is required for the regulation of melanin and pectinase production, thereby contributing to fungal development and pathogenicity (Wu et al., 2017). VelB of C. lunata is proposed to be involved in the regulation of mycelia melanization, the oxidative stress response and is essential for its pathogenicity, as CIVelB plays a major role in regulating M5HF2C production, an important virulence factor in C. lunata (Gao et al., 2017). Taken together, VelB (in complexes with other velvet domain proteins) is required for the regulation of various processes, including control of developmental programs and pathogenicity, in filamentous fungi.

VelC, as the fourth member of the velvet domain proteins, seems to be an auxiliary control factor in $A$. nidulans, which has been less studied than the other velvet domain proteins. Deletion of velC leads to an increase in conidiation and reduced number of cleistothecia, whereas the overexpression enhances sexual development (Park et al., 2014). VelC is proposed to regulate sexual development, although the exact mechanism is not known so far (Park et al., 2014). In vitro analyses showed that VelC is another potential interaction partner of VosA. The VelC-VosA complex might trigger the process of sexual fruiting body formation, as VelC is expressed at early stage of sexual development and it is also possible that the VelC protein plays a role in activating sexual development (Park et al., 2014).

\subsubsection{The fungal-specific velvet domain regulators are structurally similar to NF-KB}

The velvet family of regulatory proteins share the fungal specific and highly conserved velvet domain (FIGURE 4) comprising around 150 amino acids (aa), which is considered as protein interaction surface as well as DNA-binding domain (Ahmed et al., 2013). The velvet domain proteins modulate expression of target genes through the formation of homo- and heterodimers. These dimers specifically recognize and bind to different promoter regions, leading to activation or repression of certain target genes (Ahmed et al., 2013; Calvo et al., 2016). 


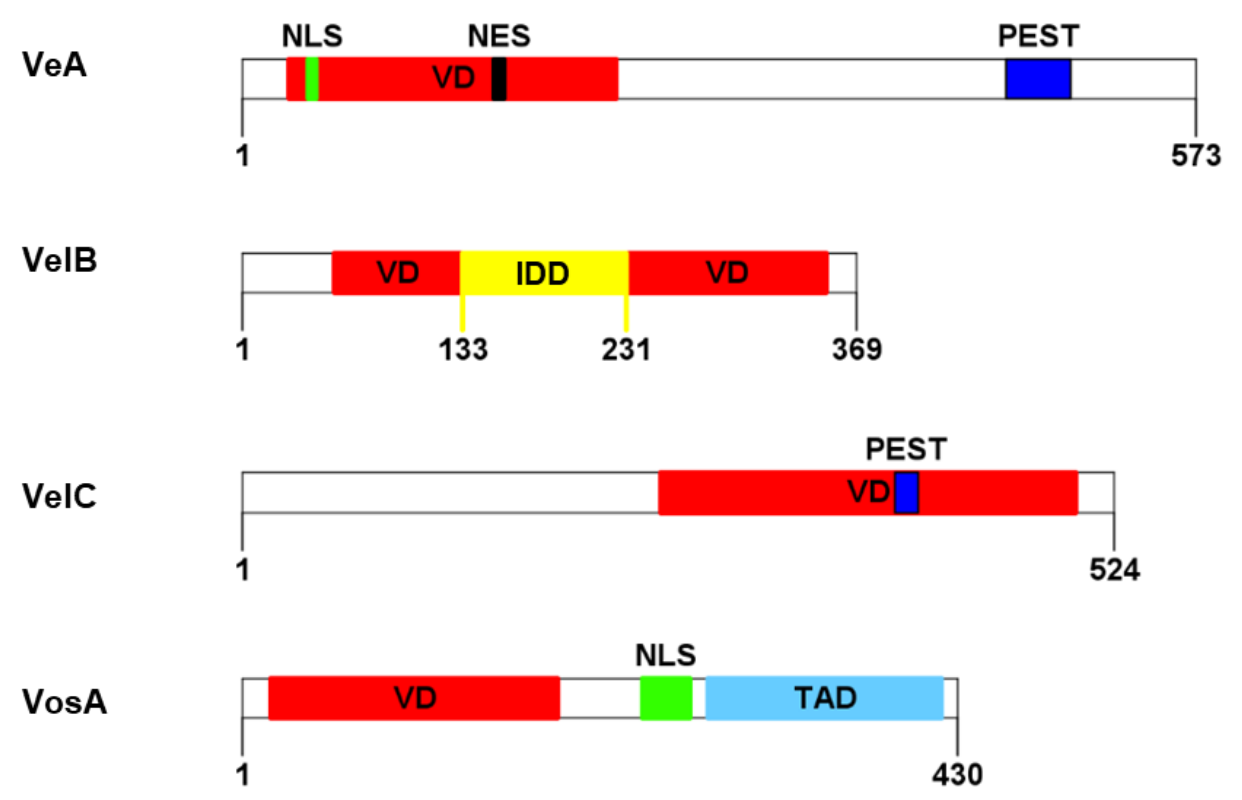

FIGURE 4: Domain architecture of $\boldsymbol{A}$. nidulans velvet proteins.

The velvet domain proteins are characterized by the velvet domain (VD). Numbers indicate amino acid residues. NLS: nuclear localization signal, NES: nuclear export sequence, IDD: intrinsically disordered domain, PEST: sequence rich in proline $(\underline{P})$, glutamate $(\underline{E})$, serine $(\underline{S})$ and threonine ( $\underline{T})$, TAD: transactivation domain (modified from Gerke and Braus, 2014).

The sequence of the velvet domain did not show significant sequence similarity to any known protein domain so far, but the crystal structure of the heterodimer of VelB and truncated Vos $A_{1}$ 190, comprising the velvet domain, revealed a structural similarity of the velvet domain to the Rel homology domain (RHD) of the mammalian transcription factor family NF-kB (FIGURE 5). Both domains share a sequence similarity of 13.7\% (Ahmed et al., 2013; Gerke and Braus, 2014).

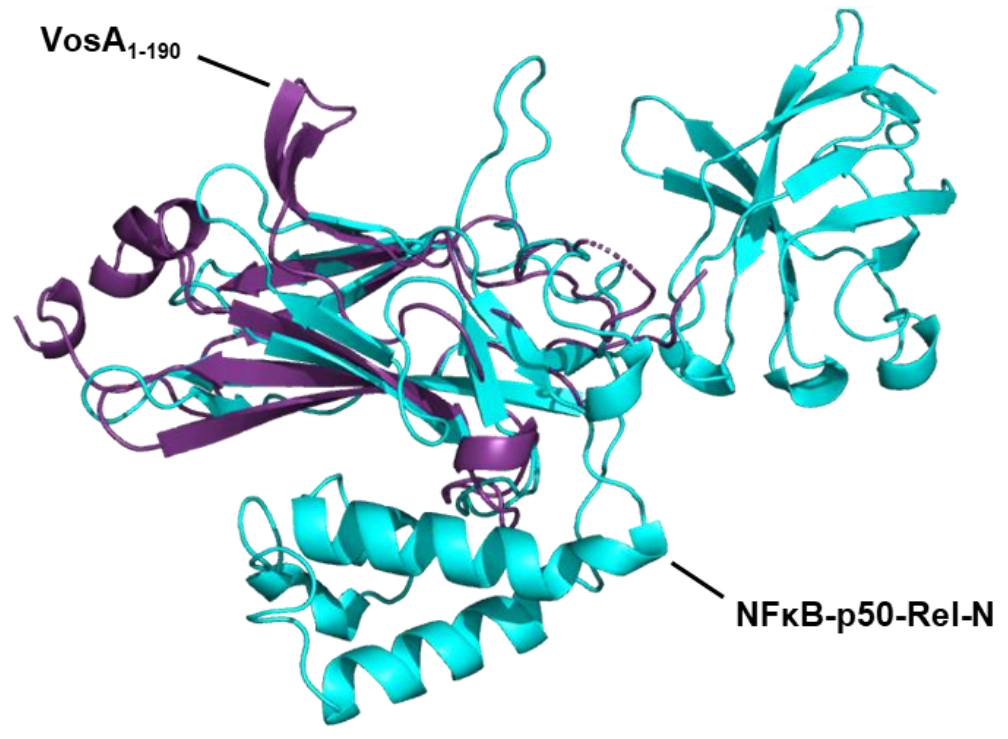

FIGURE 5: The Vos $A_{1-190}$ velvet domain crystal structure is similar to the NF-kB Rel homology domain structure.

Superposition of the crystal structures of the $\operatorname{Vos}_{1-190}$ velvet domain (PDB ID: 4N6R) and the NF-kB Rel homology domain (PDB ID 1SCV) reveals their structural similarities. 
NF-KB family proteins, similar to the velvet domain regulators in filamentous fungi, regulate transcription of target genes through combinatorial formation of dimers, which are controlled by a stimulus-responsive signaling system (Ahmed et al., 2013; Gerke and Braus, 2014; O'Dea and Hoffmann, 2010; Oeckeninghaus et al., 2011; Tao et al., 2014). In the Rel homology domain, the DNA recognition loop is at the N-terminus (Rel-N), whereas the C-terminus (RelC) is characterized as dimerization domain (Müller et al., 1995). The fold of NF-kB Rel-N resembles that of the VosA velvet domain (FIGURE 5). The critical residues for DNA-binding could be identified by comparing the overall structure of VelB-VosA with NF-kB. A loop with a patch of positively charged residues binds to the major groove of the DNA (Ahmed et al., 2013).

\subsubsection{Domain architecture of velvet proteins}

VeA is composed of 573 amino acids (aa). The velvet domain is located at the N-terminus of the protein and includes a bipartite nuclear localization signal (NLS) and a nuclear export signal (NES), allowing the protein to be shuttled between cytoplasm and nucleus (FIGURE 4) (Kim et al., 2002; Rauscher et al., 2016; Stinnett et al., 2007). Furthermore, a region enriched with proline $(\mathrm{P})$, glutamic acid I, serine $(\mathrm{S})$ and threonine $(\mathrm{T})$ residues, denominated as PEST motif, is located at the C-terminus of the protein. This motif is suggested to be a signal peptide for protein degradation (Rogers et al., 1986; Spencer et al., 2004).

VosA was discovered during a gain-of-function genetic screen ( $\mathrm{Ni}$ and $\mathrm{Yu}, 2007$ ). It contains 430 aa and harbors the velvet domain at the N-terminus like VeA and a NLS in the middle of the protein (FIGURE 4) (Shin et al., 2015; Yu et al., 2010). Furthermore, VosA carries a transcription activation domain (TAD) located at the C-terminus, which was originally leading to the suggestion that VosA might act as a transcription factor (Bayram et al., 2008; Ni and $\mathrm{Yu}$, 2007; Park et al., 2012b). Ahmed and co-workers could show in 2013 that VosA binds to more than 1,500 genes in $A$. nidulans and specifically recognizes and binds an 11-nucleotide consensus sequence in the promoter regions of key developmental regulatory genes (Ahmed et al., 2013; Bayram and Braus, 2012). VelC consists of 524 aa and contains the velvet domain at the C-terminus, which also harbors a PEST motif (FIGURE 4) (Gerke and Braus, 2014; Park et al., 2014).

VelB is the smallest member of the family of velvet domain proteins of $A$. nidulans and comprises 369 aa and the velvet domain is located more close to the C-terminus (Bayram et al., 2008; Park et al., 2012b). In contrast to VeA, VelC and VosA, the VelB velvet domain is interrupted by 99 amino acids in A. nidulans (FIGURE 4) (Gerke and Braus, 2014). These 99 residues interrupting the velvet domain of VelB are not present in the crystal structure of the $A$. nidulans $V_{0 s} \mathrm{~A}_{1-190}$-VelB heterodimer, which was recombinantly expressed and purified from E. coli (FIGURE 6, black circle) (Ahmed et al., 2013). This is probably due to enzymatic 
cleavage, as for crystallization approaches a protease treatment was performed (Ahmed et al., 2013). The structure of this region is therefore not accessible and the region is predicted as an intrinsically disordered domain (IDD).

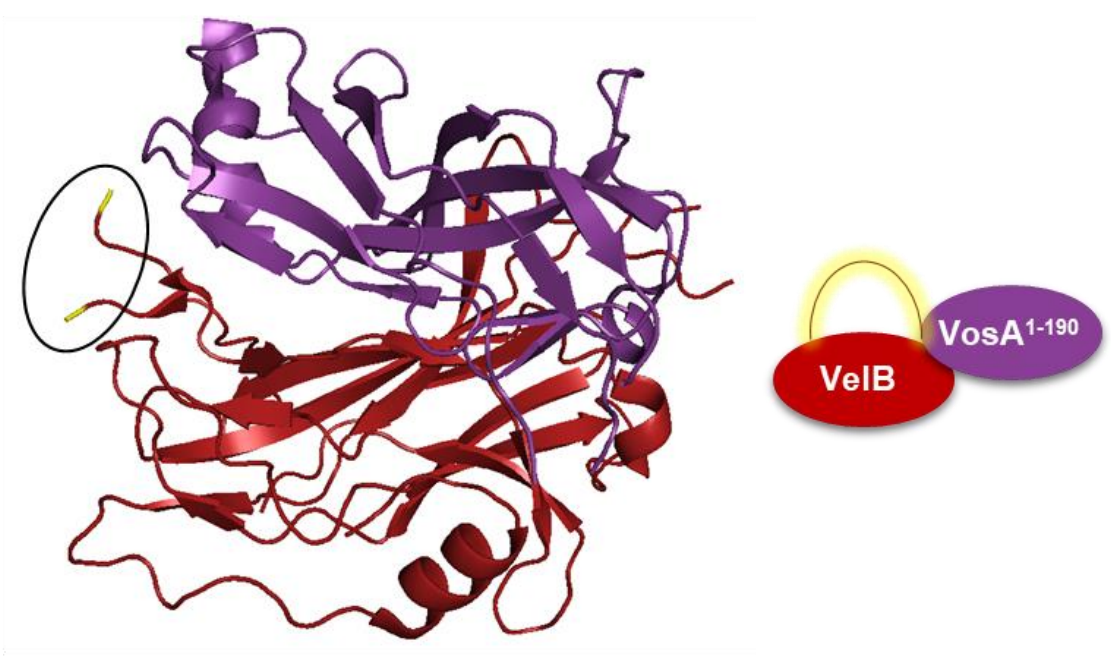

FIGURE 6: The VelB intrinsically disordered domain is absent in the crystal structure of the VelB-Vos $A_{1-190}$ heterodimer of $A$. nidulans.

The interaction of the velvet domains results in the formation of the VelB-Vos $A_{1-190}$ heterodimer. 99 amino acids, which are inserted into the velvet domain of VelB, are absent in the crystal structure, which were cut most likely by protease treatment during crystallization. This region is predicted to be an intrinsically disordered domain (IDD) (black circle). The black circle shows the connections (yellow) of the IDD to the VelB velvet domain (red).

\subsubsection{Intrinsically disordered domains}

The classical protein structure-function paradigm suggested that the well-defined and folded three-dimensional protein structure is required for the function of a protein (Lee et al., 2015; Sigalov, 2016). Evolutionary relationships can be unraveled between structurally similar proteins, diverged in their amino acid sequence. Protein sequences lacking bulky hydrophobic amino acids are incapable to build a well-organized hydrophobic core that constitutes a structured domain (Romero et al., 2001; Uversky et al., 2000). These segments, which hardly form a defined three-dimensional structure, are considered as intrinsically disordered (Lee et al., 2015). Proteins without structural constrains, in contrast to protein domains with a defined structure, tend to evolve rapidly and homologous regions are harder to identify for unstructured than for structured domains (Bellay et al., 2011; Brown et al., 2009; Chen et al., 2006). This results in a complication of transferring the functional information between homologs and consequently the predicted function of intrinsically disordered domains.

Customarily, these intrinsically disordered regions were considered as passive "linkers", connecting structured domains within a protein (Lee et al., 2015). The last two decades research in protein structure regarding order and disorder revealed the widespread role of 
intrinsically disordered domains in biological processes (Latysheva et al., 2015). Disordered domains are very common in eukaryotic proteins and most of them contain both, structured and intrinsically disordered domains (FIGURE 7) (Dunker et al., 2002, 2013; Wright and Dyson, 2015). The active participation of intrinsically disordered protein sequences is well established in protein function, extending the traditional structure-function paradigm.

One example for a disordered protein is the conservative presynaptic protein $\alpha$-synuclein. Aggregation and fibrillation of $\alpha$-synuclein is supposed to be involved into the pathogenesis of Parkinson's disease in human and its aggregation, promoted by tyrosine nitration, confers toxicity to yeast (Kleinknecht et al., 2016). a-synuclein is thought to be able to adopt different secondary structures depending on its environment. This chameleon behavior might allow this protein to remain substantially unfolded or to adopt an amyloidogenic partially folded structure, or to build $\alpha$-helices or $\beta$-strand sheets (Oldfield et al., 2008; Uversky, 2003). Another versatile intrinsically disordered protein is Sem1, which was originally identified in S. cerevisiae. It is associated with many functionally different protein complexes where it also acts as assembly supporter without being part of the final active complex itself and it was shown recently that Sem1 is required for accurate $26 \mathrm{~S}$ proteasome assembly in $A$. nidulans (Kolog Gulko et al., 2018; Kragelund et al., 2016).

Disordered domains play a central role in regulation of signaling pathways and the control of transcription and translation in protein interaction networks (Wright and Dyson, 2015). Proteins containing segments of intrinsic disorder act as hubs in protein interaction networks. Complex formation with multiple different partners across time and space supports the coordination of several cellular activities. There are different possibilities how protein function can be regulated through disordered domains: (1) the flexibility of intrinsically disordered domains increases the

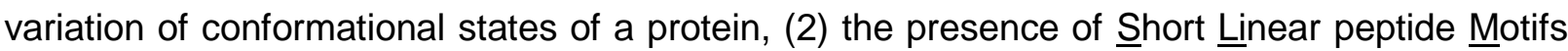
(SLiMs) enables a heterogeneity in the protein interaction surface and (3) posttranslational modifications alter the chemical nature of the polypeptide and lead to an increase of interaction partners (Babu et al., 2012; Buljan et al., 2013; Gsponer and Babu, 2009; Mao et al., 2013). 
Structure-function paradigm

Sequence

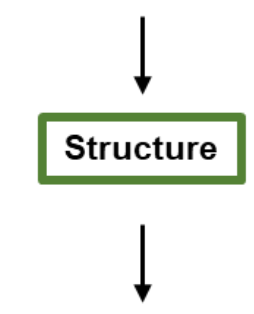

Function

e.g. enzyme catalysis
Disorder-function paradigm

Sequence

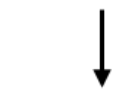

Disorder<smiles>[13CH3]</smiles>

Function e.g. DNA or protein-binding

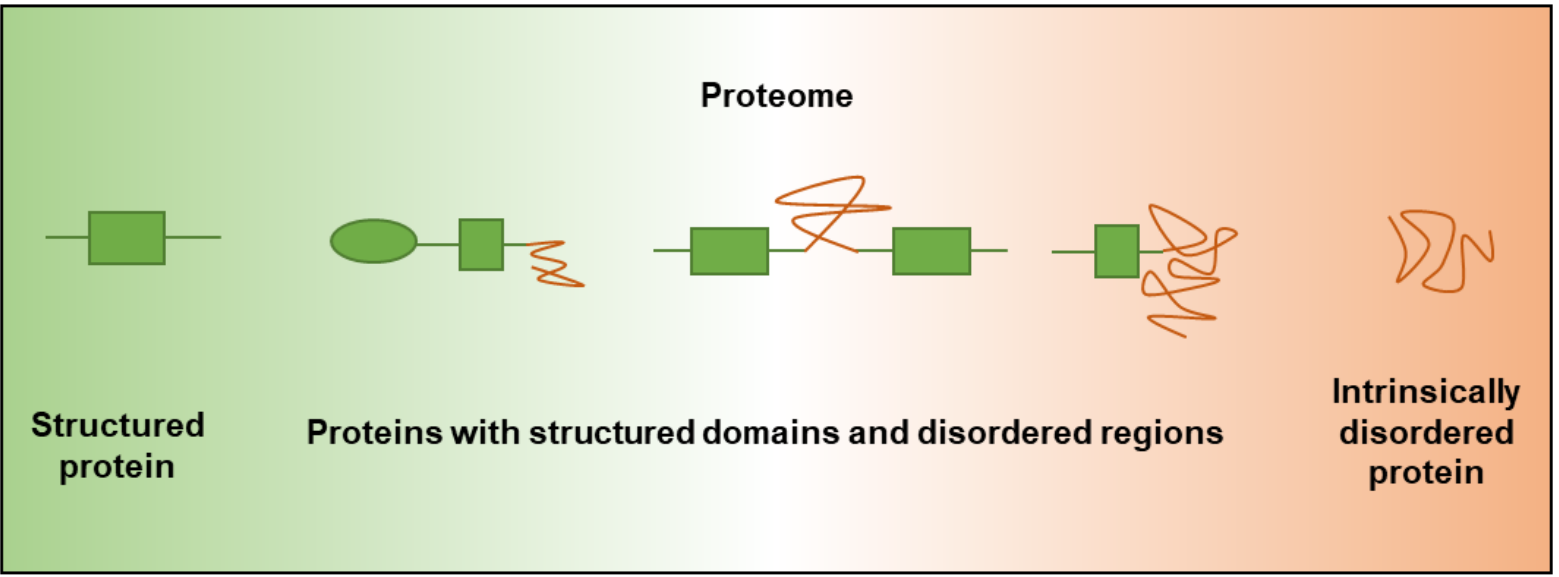

FIGURE 7: Most of eukaryotic proteins contain structured domains und intrinsically disordered regions.

Two fundamental building blocks of proteins are represented by structured domains and intrinsically disordered regions. The synergy of these building blocks extends the functional versatility of proteins (modified from Babu et al., 2012; Lee et al., 2015).

It has been reported for yeast, mouse and human proteins that the presence of a terminal or internal sequence which is intrinsically disordered, leads significantly shorter half live of these proteins compared to structured proteins. This is presumably due to the disordered regions, which can promote the initiation of degradation by the proteasome (van der Lee et al., 2014). An accurate control of protein turnover is fundamental for cellular homeostasis. Alteration in protein half-life resulting in an imbalance of relative protein levels can cause abnormal development and diseases (Ciechanover, 2012).

\subsection{Protein degradation in eukaryotes}

Cellular protein homeostasis is achieved by balancing protein biosynthesis and degradation and is required to maintain and coordinate growth and cell division during the cell cycle (He and Klionsky, 2009; Nam et al., 2017). Protein synthesis is controlled at the transcriptional 
level and two major pathways resulting in the degradation of proteins exist in eukaryotes. Autophagy, which can occur as unspecific or also as highly specific degradation pathway, is the first choice to recycle intra- or extracellular proteins mediated by a highly regulating selfeating process under unfavorable conditions, such as nitrogen or glucose starvation (FIGURE 8) (Onodera and Ohsumi, 2005; Szilágyi et al., 2011). Single proteins, complexes and also whole organelles are engulfed by autophagosomes, which transport the cargo to the lysozyme (animals) or its equivalent, the vacuole (fungi and plants) (He and Klionsky, 2009; Onodera and Ohsumi, 2005).

The other major pathway for protein breakdown is mediated by ubiquitin, a small protein attached to other proteins to label them for degradation by the $26 \mathrm{~S}$ proteasome. The ubiquitin proteasome system (UPS) is highly conserved in eukaryotes, controlling the half-life of regulatory proteins to ensure accurate regulation of the cell cycle, development, stress response, signal transduction and DNA maintenance (Ciechanover, 1998; Jöhnk et al., 2016; Kleiger and Mayor, 2015). The UPS can be subdivided into two mechanisms. The first system guarantees the quality control of newly synthesized proteins and is termed endoplasmic reticulum associated degradation (ERAD) (FIGURE 8). Ubiquitin ligases, embedded in the ER, identify and ubiquitinate misfolded or mutated proteins and transport them to the $26 \mathrm{~S}$ proteasome for degradation (Christianson and Ye, 2014; Hirsch et al., 2009). Accumulation of potentially toxic or misfolded proteins in the ER contributes to ER stress. ER homeostasis is ensured by the unfolded protein response system, which activation results for instance in the induction of the expression of genes important for protein folding (Hampel et al., 2016; Heimel, 2014).

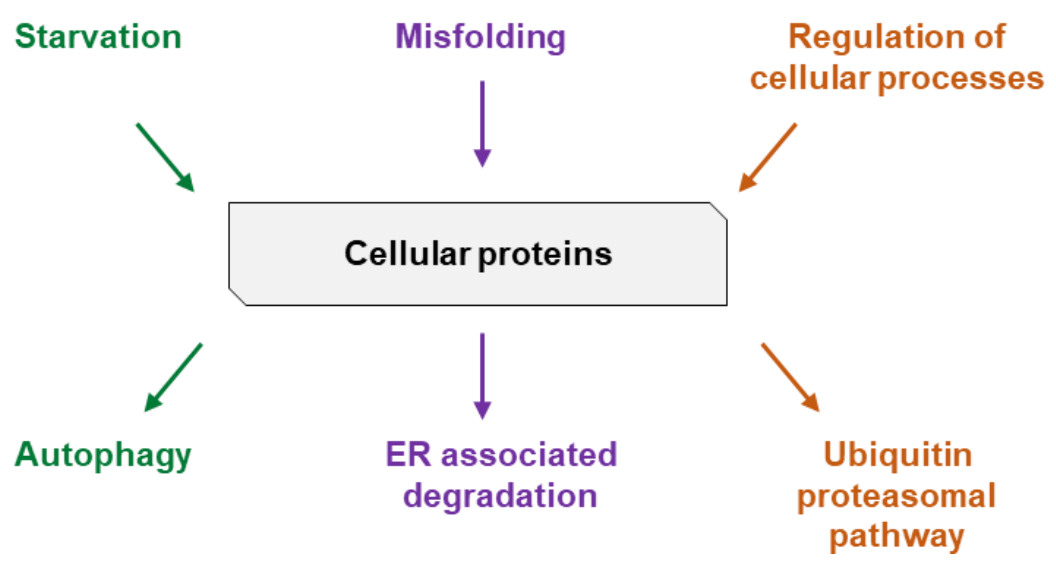

FIGURE 8: Protein degradation is achieved by different mechanisms in eukaryotes.

The protein pool within an eukaryotic cell is controlled and degraded by different mechanisms. Starvation results in protein recycling via autophagy, misfolding of proteins triggers ER associated degradation and the specific regulation of cellular processes is achieved by the ubiquitin proteasome pathway. 
The ubiquitin proteasome pathway is the second mechanism, which is required for ubiquitination and immediate degradation of specific intracellular proteins, contributing to the accurate regulation of cellular processes like development and stress response (FIGURE 8) (Ciechanover, 1998; Jöhnk et al., 2016; Meister et al., 2016).

Ubiquitinated proteins are mainly associated with the UPS but during the last years several studies showed that ubiquitin is also involved in selective autophagy processes (Ashida et al., 2014; Kleiger and Mayor, 2015; Nam et al., 2017; Schreiber and Peter, 2014). Selective autophagy is required for intracellular homeostasis, cell differentiation, development, defense, cell survival and cell death and defects in this process in mammals result in neurodegenerative, inflammatory and cardiovascular diseases or cancer (Schreiber and Peter, 2014; Zaffagnini and Martens, 2016). The decision whether ubiquitinated proteins are degraded by the autophagosome or by the 26S proteasome depends on the procedure with which single ubiquitin proteins are attached to one another. Ubiquitin chains, which develop from ubiquitin residues linked to the lysine 63 residue of the preceding ubiquitin molecule, are mainly recognized by ubiquitin binding autophagy receptors, inducing the autophagy-lysosomal degradation pathway. Lysine 48 linked ubiquitin chains in contrast are directed to the $26 \mathrm{~S}$ proteasome (Kirkin et al., 2009; Komander and Rape, 2012; Meister et al., 2016).

\subsubsection{The ubiquitin-proteasome pathway}

A highly sensitive protein degradation mechanism is determined by the ubiquitin proteasome pathway, which involves two steps: 1) multiple ubiquitin units are covalently attached to the substrate as a chain and 2) the marked substrate is degraded by the $26 \mathrm{~S}$ proteasome with release of the ubiquitin, which gets recycled (Glickman and Ciechanover, 2002). Ubiquitin is highly evolutionary conserved and consists of 76 amino acids and at least four monomers have to be linked to each other and attached to the substrate to label it for degradation by the $26 S$ proteasome (Glickman and Ciechanover, 2002). The attachment of the ubiquitin molecules is achieved via a tree-step cascade, involving the E1, E2 and E3 enzymes (FIGURE 9). First, ubiquitin is activated in an ATP-dependent manner by a high-energy thioester linkage between the C-terminal glycine residue of ubiquitin and a cysteine residue of the ubiquitinactivating enzyme E1. This is followed by the transfer of the ubiquitin to an ubiquitinconjugating enzyme E2. In a final step, the ubiquitin is transferred from the E2 enzyme and covalently attached to a lysine residue of a specific protein substrate with a E3 ubiquitin ligase (FIGURE 9) (Ciechanover, 1998; Glickman and Ciechanover, 2002). The subsequent rounds of the E1-E2-E3 cascade enable the assembly of an ubiquitin chain onto the substrate, marking it for degradation. Thereby, a new ubiquitin is covalently attached to a former linked ubiquitin via the internal lysine residue 48. The E1 enzyme is highly conserved within eukaryotes, whereas there are many species of E2 and multiple families of E3 ligases or E3 multiprotein complexes 
(Hersko and Ciechanover, 1998). For instance, in the mammalian E1-E2-E3 cascade only two E1 ubiquitin-activating enzymes exist, transferring the ubiquitin molecule to one of approximately 40 E2 ubiquitin-conjugating enzymes, which pass ubiquitin to more than 600 known E3 ligases (Deshaies and Joazeiro, 2009; Kleiger and Mayor, 2015).

The transfer of ubiquitin to the substrate protein is performed by two different types of E3 ligases: the homologous to the E6-AP carboxyl terminus (HECT) domain and the really interesting new gene (RING)-domain use different mechanisms to transfer ubiquitin to the protein substrate (Ho et al., 2006). The HECT E3 ligases include an additional intermediate step prior to the labelling of the substrate, where the activated ubiquitin is transferred to an active cysteine residue within the HECT E3 ligase and then targeted to the protein substrate. In contrast, RING E3 ligases directly transfer the ubiquitin from the E2 enzyme to the substrate (Glickman and Ciechanover, 2002).

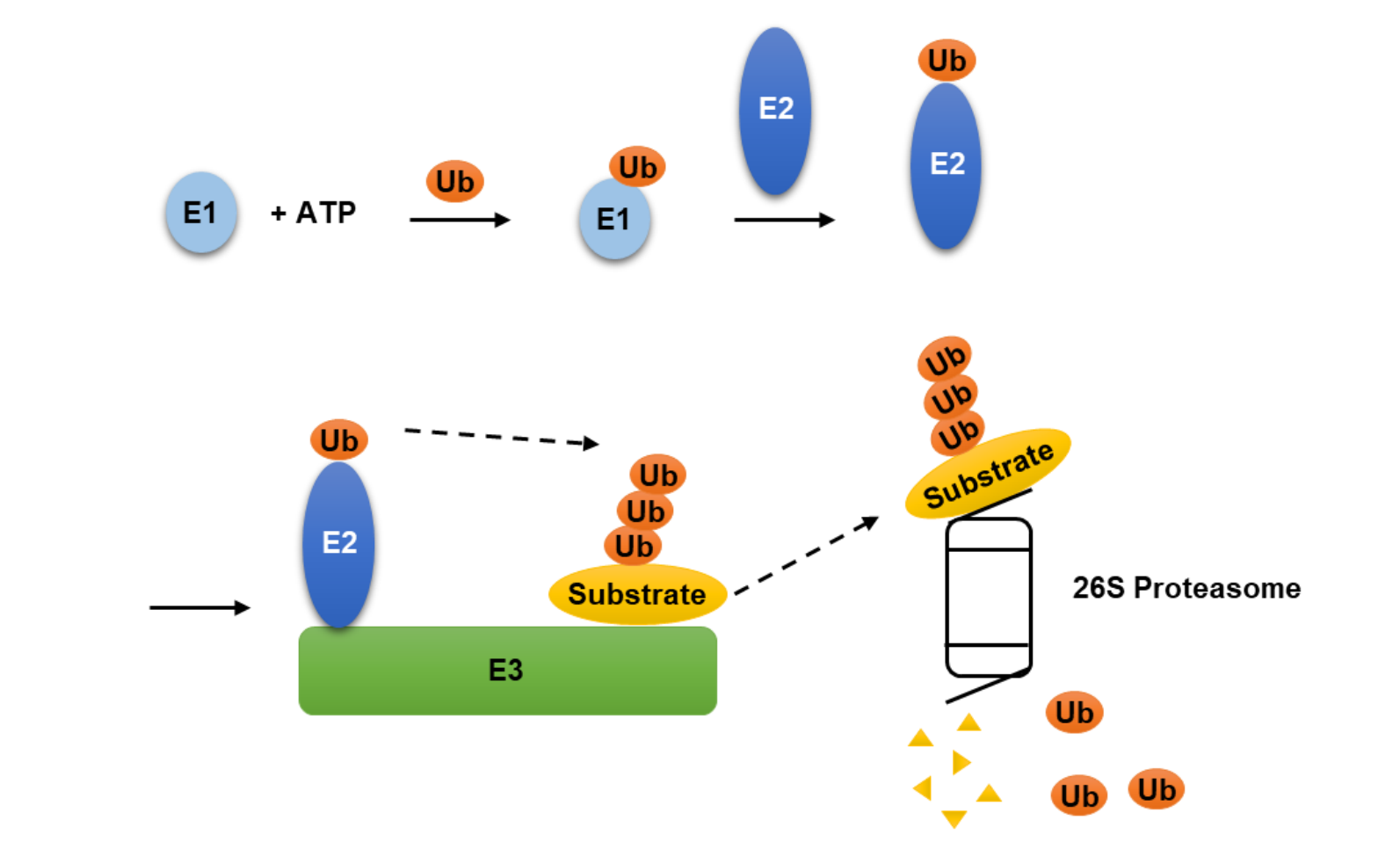

FIGURE 9: The ubiquitin proteasome pathway.

Ubiquitin is activated in an ATP-dependent manner by the ubiquitin-activating enzyme E1 and transferred to the E2 ubiquitin-conjugating enzyme. Finally, the activated ubiquitin is covalently attached to a lysine residue of a specific protein substrate within the E3 ubiquitin ligase, resulting in degradation by the $26 \mathrm{~S}$ proteasome.

\subsubsection{Cullin-RING E3 ligases}

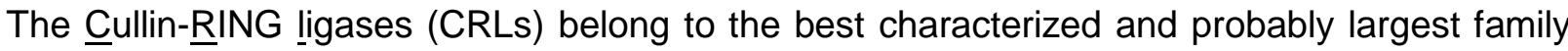
of E3 ubiquitin ligases (Petroski and Deshaies, 2005; Schinke et al., 2016; von Zeska Kress et al., 2012). Cullins, a family of hydrophobic proteins, serve as scaffold within the CRLs and are 
evolutionary conserved (Bosu and Kipreos, 2008). The mammalian genome encodes genes for eight cullins (CUL1, CUL2, CUL3, CUL4A, CUL4B, CUL5, CUL7 and PARC), whereas lower eukaryotes possess less cullins. The nematode Caenorhabditis elegans has six cullins, Drosophila melanogaster and Arabidopsis thaliana have five cullins and most fungi contain three cullins (CulA, CulB, CulC) (Sarikas et al., 2011; Skaar et al., 2007). The cullin scaffold is able to bind to the RING-domain containing protein with its cullin homology domain at the Cterminus and to a subset of specific substrate adaptors at the N-terminus. The Skp-Cullin-Fbox (SCF)-complex belongs to the best characterized CRLs. This complex is composed of four major components in $A$. nidulans: $\underline{S}$ phase kinase-associated protein $\underline{A}$ (SkpA), $\underline{\text { Cullin}} \underline{A}$ (CulA/Cul1), the $\underline{R} I N G-\underline{b} 0 \underline{x}$ protein $\underline{A}$ RbxA and an F-box protein (FIGURE 10). CulA acts as a scaffolding protein for the assembly of the whole complex and interacts with SkpA and RbxA, thereby forming the core complex. SkpA binds to an F-box protein as substrate adaptor, which determines the substrate specificity within the complex (Meister et al., 2016; Petroski and Deshaies, 2005; von Zeska Kress et al., 2012). RbxA presents the linker protein between CulA and the ubiquitin-conjugating enzyme E2 and carries the activated ubiquitin for the transfer of the terminal carboxyl-group of ubiquitin to the $\varepsilon$-amino group of a lysine residue of the substrate (FIGURE 10). The diversity of CRLs is, besides the variation of cullin subunits for CRL assembly, conferred by different F-box proteins.
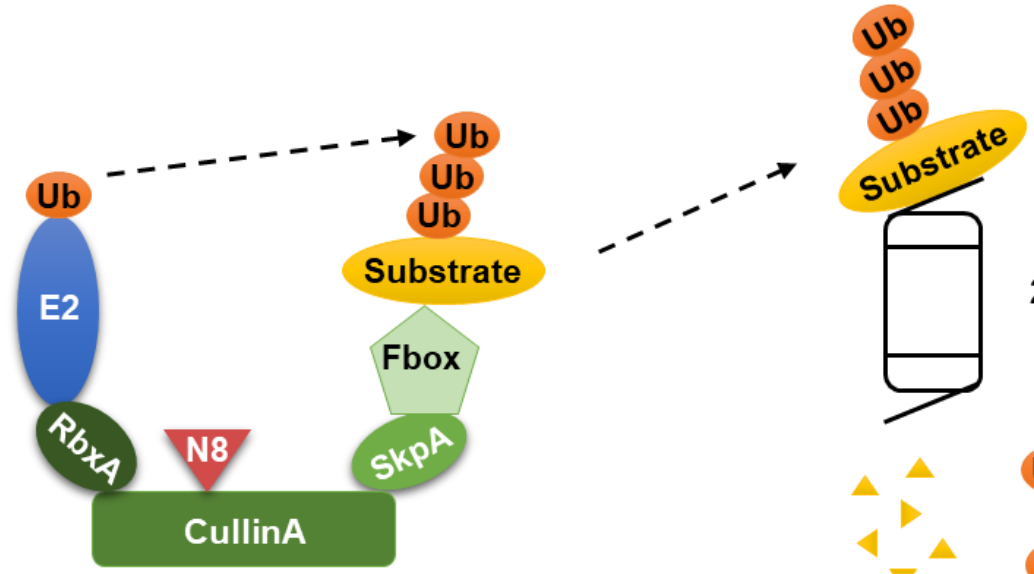

26S Proteasome

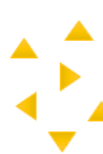

Ub

Ub Ub

\section{FIGURE 10: The SkpA-CullinA-F-box complex of $A$. nidulans.}

The SkpA-CullinA-F-box (SCF) complex is one of the best characterized Cullin RING ligases. The RING finger protein RbxA links the E2 conjugating enzyme to the CulA scaffold, which is also connected to the adaptor protein SkpA. SkpA binds to an F-box protein, determining the substrate specificity. The substrate is polyubiquitinated $(\mathrm{Ub}=$ ubiquitin) and degraded by the $26 \mathrm{~S}$ proteasome. $\mathrm{N} 8=\mathrm{Nedd} 8$ promotes the transfer of ubiquitin to a substrate. 
The activity of CRLs is regulated by the covalent attachment of Nedd8 (neural precursor cell expressed, developmentally down-regulated $\underline{8}$ ) to a lysine of cullins. This process is highly related to ubiquitination and is termed neddylation (Schinke et al., 2016; von Zeska Kress et al., 2012). Neddylation leads to a conformational change of the CRL that promotes the transfer of ubiquitin to a substrate, which results in its degradation (Duda et al., 2008). Neddylation and its reverse process deneddylation, enable the modularity of CRLs, allowing ubiquitin labelling of a plethora of proteins, resulting in their degradation by the $26 \mathrm{~S}$ proteasome (Meister et al., 2016; Schinke et al., 2016). Two deneddylases are described in eukaryotes, the deneddylase 1 (Den1, corresponding to DenA in $A$. nidulans) and the constitutive photomorphogenesis factor $\underline{9}$ (COP9), called $\underline{\text { COP9 }}$ signalosome (CSN), (Christmann et al., 2013; Schinke et al., 2016). The substrate adaptor of CRLs can be exchanged by the binding and regulation of the cullin-âssociated Nedd8-dissociated 1 Cand1 protein. In most eukaryotes Cand1 exists as a single protein but in Aspergillus species Cand $\mathrm{A}$ is composed of two proteins, CandA-N and CandA-C, resembling the smaller $\mathrm{N}$-terminal and the larger $\mathrm{C}$-terminal part of Cand1 (Helmstaedt et al., 2011). Subsequently, the Cand1 protein can be exchanged by new substrate adaptors, in SCF complexes by F-box proteins, and CRLs can be reactivated by neddylation (Beckmann et al., 2015; Helmstaedt et al., 2011; Meister et al., 2016; Olma and Dikic, 2013; Schinke et al., 2016). The major ubiquitin-dependent protein degradation machinery in the cytoplasm and the nucleus is represented by the $26 \mathrm{~S}$ proteasome, which consists of a barrel-like 20S core particle, which is capped with one or two 19S regulatory particles (Peters et al., 1994, Kolog Gulko et al., 2018). The $20 S 700$ kDa core particle includes the proteolytic active site and possesses two entrance pores. These pores enable unfolded proteins to pass, whereas native proteins cannot enter. The 195 regulatory particles ensure additional regulation and are connected to the ends of the $20 \mathrm{~S}$ core particle (Bhattacharyya et al., 2014).

\subsubsection{F-box proteins}

F-box proteins function as substrate-adaptors and determine the substrate-specificity within the SCF complex (Skaar et al., 2013). They are characterized by the N-terminal F-box domain, comprising 40-50 amino acids, which was first discovered in the mammalian F-box protein cyclin F, (Bai et al., 1996). The F-box domain mediates the protein-protein interaction with the CulA associated SkpA, enabling the formation of the SCF complex (Jöhnk et al., 2016; Stefanowicz et al., 2015). Almost all F-box proteins contain a highly conserved proline residue at position seven of the F-box domain, often with a preceding leucine residue at position six (FIGURE 11) (Schmidt et al., 2009). 


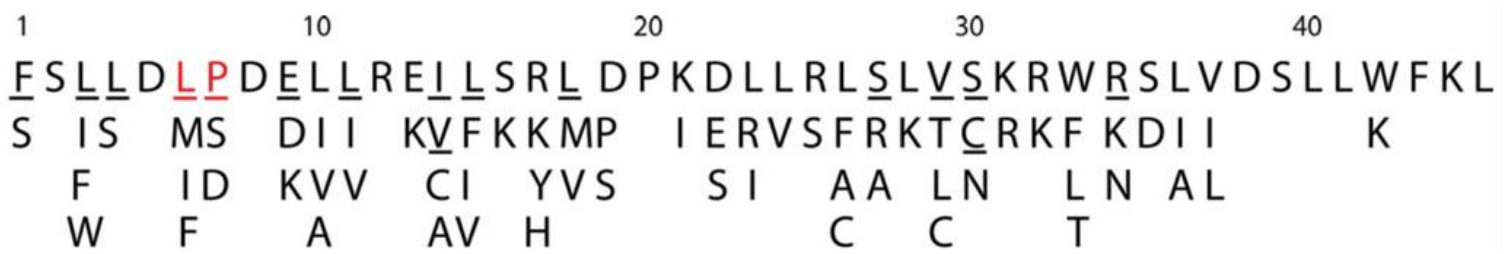

FIGURE 11: The F-box domain contains a highly conserved proline and leucine residue. The F-box consensus motif comprises about 45 amino acids based on the Hidden Markov model (HMM) of the F-box domain (PF00646). Amino acids that are highly conserved are underlined and the most conserved ones, proline $(P)$ at position seven and leucine $(L)$ at position six are highlighted in red. Amino acid residues are given in one letter code. Amino acid residues are sorted at each position from top to bottom by decreasing occurrence in F-box proteins (adapted from Jonkers and Rep, 2009).

This proline residue is required for the incorporation of the F-box protein into the SCF complex by binding to the SkpA adaptor (Schmidt et al., 2009). F-box proteins contain an additional protein interaction domain at the C-terminus, which serves as substrate binding site. Due to the substrate-specific domain at the $\mathrm{C}$-terminus, F-box proteins can be divided into three main classes (Skaar et al., 2013; Stefanowicz et al., 2015):

- FBXW: F-box proteins containing WD-40 domains, which comprise WD-40 repeats. These are short structural motifs consisting of about 40 amino acids, often ending with a tryptophan-aspartic acid (W-D) dipeptide and which have to be phosphorylated on its serine residues (Neer et al., 1994).

- FBXL: F-box proteins that contain LRR domains. They are built of leucine rich repeats (LRR), a short structural motif, consisting of repeating 20-30 amino acid stretches. The LRR domain forms an $\alpha / \beta$ horseshoe fold and often also requires the phosphorylation of target proteins for efficient binding (Enkhbayar et al., 2004; Kobe and Deisenhofer, 1994).

- FBXO: F-box proteins, which were originally categorized as F-box only, but contain other C-terminal domains, such as Kelch domains, Armadillo and tetratricopeptide repeats (TPR), zinc fingers, proline-rich or unknown motifs (Jin et al., 2004).

The substrate recruitment is defined by the rapid and specific binding of F-box proteins to their targets and represents the ultimate regulation of SCF complex activity. The specific to be degraded target protein requires short and defined amino acids sequences to be recognized by the F-box protein. This sequence is referred to as the degradation motif or degron. F-box proteins belonging to the FBXW or FBXL family usually bind to phosphorylated targets by interaction through so-called phosphodegrons. The best characterized and $\underline{\beta}$-transducin repeat-containing F-box proteins ( $\beta$ TrCP) Fbxw1 and Fbxw7 bind to phosphodegrons, in which the threonine and serine residues are phosphorylated (Skaar et al., 2013). The highly specific and regulated substrate recruitment of the F-box proteins is achieved by different mechanisms. 
The recognition of the target proteins might depend on a co-factor or the degron can be blocked by modification or limited access. Also the localization of either the substrate or the F-box protein itself can control the substrate recruitment (Stefanowicz et al., 2015).

The number of F-box proteins and their function varies within eukaryotes. About 700 in the flowering plant Arabidopsis thaliana and 14 F-box proteins in the yeast Saccharomyces cerevisiae were identified, whereas humans possess about 69 F-box proteins and 74 F-box proteins where identified in A. nidulans (Draht et al., 2007; Hua and Vierstra, 2011). F-box proteins are involved in control of the cell division cycle, glucose sensing, mitochondrial connectivity, and control of the circadian clock in fungi (Jonkers and Rep, 2009). The F-box protein FWD1 of Neurospora crassa is required for the degradation of the circadian clock regulator protein FRQ1 (He et al., 2003). CDC4 of S. cerevisiae is an F-box protein conferring to the regulation of multiple processes, such as the control of the cell division cycle, morphogenesis, nutrient sensing and calcium signaling (Jonkers and Rep, 2009). Several F-box proteins in A. nidulans play a role in development. For example, the GrrA F-box protein, which is the functional ortholog of $S$. cerevisiae GRR1 (Glucose Repression-Resistant 1 ), is required for sexual development and the grrA deletion mutant is not able to produce mature ascospores due to a block in meiosis (Krappmann et al., 2006a). Deletion of fbx23 results in constitutive sexual development, also not repressed under illuminating conditions. In contrast, a strain with a deletion of $f b \times 15$, is abolished in sexual and also drastically reduced in asexual development (von Zeska Kress et al., 2012).

F-box proteins, beside their role as substrate receptor within the SCF complex, have been shown to present functions, which are independent of the SCF complex. Fbx15 of A. fumigatus is not only part of the SCF complex, but is required for the control of the nuclear localization of SsnF/Ssn6, a component of the highly conserved eukaryotic transcriptional Ssn6/Tup1 corepressor complex (Jöhnk et al., 2016). 


\subsection{Aim of this study}

The aim of the study was to analyze the influence of the VelB intrinsically disordered domain on selective heterodimer formation and its role in development and secondary metabolism in A. nidulans.

The fungal specific velvet regulatory proteins are characterized by the velvet domain, encompassing approximately 150 amino acids, which enables protein-protein interaction and the binding to DNA (Ahmed et al., 2013; Gerke and Braus, 2014). The VelB velvet domain is, in contrast to the velvet domain of VeA, VelC and VosA, interrupted by a 99 amino acid region of intrinsic disorder in $A$. nidulans. The timely and spatially controlled formation of different velvet domain protein dimers is required for the regulation of developmental programs and the specific interconnected production of secondary metabolites in response to environmental stimuli. The VelB-VeA heterodimer is indispensable for the induction of sexual development, whereas VosA, presumably as VosA-VosA homodimer, represses the asexual cycle and VelBVosA is required for the viability of sexual and asexual spores. VelB also positively influences, maybe as a homodimer, asexual development. The fungal cell therefore needs the possibility to regulate the distribution of VelB to form the different dimers and support the appropriate developmental program and the biosynthesis of certain secondary metabolites. The control of the cellular amount of the velvet domain proteins via protein synthesis and degradation is one mechanism to manage the formation of required complexes.

The present study investigated whether the interruption of the VelB velvet domain by an intrinsically disordered domain (IDD) is an additional level of control to channel available VelB to the direction of VeA or VosA. The stability of VelB with and without IDD was compared to examine whether the VeIB IDD is a target for degradation by the $26 \mathrm{~S}$ proteasome. Additionally, the influence of F-box proteins, which are the substrate receptors within SCF complexes, on the cellular VelB protein amount was investigated. GFP pull down experiments were conducted to analyze if deletion of the $v e / B^{I D D}\left(v e / B^{I D D_{\Delta}}\right)$ changes the potential of protein-protein interactions of VelB. Heterodimer formation of VelB with VeA or VosA and the homodimer formation were of peculiar interest. Phenotypical analyses and metabolite extraction of the velB $B^{I D D_{\Delta}}$ mutant strain was conducted to investigate possible changes in the developmental program and secondary metabolite production of $A$. nidulans compared to wildtype. VosA is proposed to interact with VelC in vitro and positively regulates sexual development in $A$. nidulans. GFP pull down experiments were conducted to proof the occurrence of the VosAVelC heterodimer in $A$. nidulans. Furthermore, the role of the VosA-VelC dimer in connection with the other velvet domain complexes regarding development and secondary metabolism was investigated. 


\section{Materials and methods}

\subsection{Chemicals and materials}

Buffers, solutions and media were prepared with chemicals purchased from the companies APPLICHEM GMBH (Darmstadt, Germany), BD BIOSCIENCES (Heidelberg, Germany), CARL ROTH GMBH \& CO. KG (Karlsruhe, Germany), FLUKA (Neu-Ulm, Germany), INVITROGEN (Carlsbad, CA, USA), MERCK KGAA (Darmstadt, Germany), BIOZYME SCIENTIFIC GMBH (Hessisch Oldendorf, Germany), ROCHE DIAGNOstICS GMBH (Mannheim, Germany), SIGMAALDRICH CHEMIE GMBH (München, Germany), SeRVA ElECTROPHORESIS GMBH (Heidelberg, Germany), OXOID DEUTSCHLAND GMBH (Wesel, Germany).

Plastic consumables, such as pipet tips, reaction tubes, inoculation loops etc., were purchased from SARSTEDT AG \& CO. (Nümbrecht, Germany), STARLAB GMBH (Hamburg, Germany) and NERBE PLUS GMBH (Winsen/Luhe, Germany).

Polymerases and restriction enzymes were obtained from THERMO FISHER SCIENTIFIC (Schwerte, Germany), trypsin was purchased from SERVA ELECTROPHORESIS GMBH (Heidelberg, Germany). Primers were obtained from EUROFINS GENOMICS GMBH (Ebersberg, Germany). The following primary antibodies were used in this study: GFP antibody sc-9996 was purchased from SANTA CRUZ BIOTECHNOLOGY (Santa Cruz, CA, USA), tubulin antibody T0926 from SIGMA-ALDRICH, VeIBIDD antibody from PROTEOGENIX SAS (Schiltigheim, France) and actin anti body from SigmA-ALDRICH. Furthermore, 6x-His Tag Monoclonal Antibody R93025 (THERMO FISHER SCIENTIFIC) and StrepMAB-Classic antibody 2-1507-001 (IBA) was used. As secondary antibodies horseradish peroxidase-coupled rabbit antibody (G21234, INVITROGEN) or mouse antibody (115-035-003, JACKSON IMMUNORESEARCH, Newmarket, UK) was used.

For protein purifications of GFP-tagged proteins GFP-Trap ${ }^{\circledR}$ A agarose beads from CHROMOTEK GMBH (Planegg-Martinsried, Germany) were used. For HA pulldowns Monoclonal Anti-HA-Agarose beads A2095 (SIGMA ALDRICH) were utilized.

The GeneRuler 1kb DNA ladder and the PageRuler ${ }^{\text {TM }}$ Prestained Protein Ladder (THERMO FISHER SCIENTIFIC) were used for DNA and protein on-gel band size determination. Filtropur filters with a pore size of 0.2 and $0.45 \mu \mathrm{m}$ for small-scale sterile filtration of chemicals were purchased from SARSTEDT. Ampicillin (ROTH), kanamycin (APPLICHEM), chloramphenicol (APPLICHEM), pyrithiamine hydrobromide (SIGMA-ALDRICH), clonNAT nourseothricin dihydrogen sulfate from WERNER BIOAGENTS (Jena, Germany) and phleomycin (INVIVOGEN) were used for selection of microorganisms.

DNA and protein amounts were measured with a NanoDrop ND-1000 photospectrometer from PEQLAB BIOTECHNOLOGIE GMBH (Erlangen, Germany). Agarose gel electrophoresis was 
performed with Mini-Sub ${ }^{\circledR}$ Cell GT chambers and the PowerPac ${ }^{\top M} 300$ power supply and SDSpolyacrylamide gel electrophoresis were performed with the Mini-Protean ${ }^{\circledR}$ Tetra Cell, Mini Trans-Blot ${ }^{\circledR}$ Electrophoretic Cell and powered with the PowerPac ${ }^{\top M} 3000$ from BIO-RAD LABORATORIES (Hercules, CA, USA). Proteins were transferred from SDS-polyacrylamide gels onto Amersham ${ }^{\mathrm{TM}}$ Protran $^{\mathrm{TM}} 0.45 \mu \mathrm{m}$ NC nitrocellulose blotting membranes and DNA was blotted to Amersham ${ }^{\mathrm{TM}}$ Hybond-N ${ }^{\mathrm{TM}}$ nylon membranes from GE HEALTHCARE (Little Chalfont, United Kingdom). Chemiluminescence was detected either by utilization of Amersham ${ }^{\mathrm{TM}}$ Hyperfilm $^{\text {TM }}$-ECL from GE HEALTHCARE, which were exposed with the Optimax X-ray Film Processor from PROTEC GMBH \& CO. KG (Oberstenfeld, Germany), or by exposure of the membranes with the Fusion SL chemiluminescence detector from PEQLAB. For centrifugation of 1.5 and $2 \mathrm{~mL}$ reaction tubes, Biofuge fresco (cooled) and Biofuge pico centrifuges from HERAEUS INSTRUMENTS GMBH (Hanau, Germany) were used. For centrifugation of 10, 15 and $50 \mathrm{~mL}$ centrifuge tubes Rotixa/RP from ANDREAS HETTICH GMBH \& CO. KG (Tuttlingen, Germany), 5804R from EPPENDORF AG (Hamburg, Germany) and 4K15C from SigmA LABORZENTRIFUGEN GMBH (Osterode am Harz, Germany) were used. Furthermore, Sorvall RC-3B Plus Refrigerated Centrifuge from THERMO FISHER SCIENTIFIC and Sorvall RC-5B Plus Refrigerated Centrifuge from THERMO FISHER SCIENTIFIC were used. For $\mathrm{pH}$ determination a WTW bench $\mathrm{pH} / \mathrm{mV}$ Routine meter $\mathrm{pH} 526$ (SIGMA-ALDRICH) was used. For protein purification the Äkta Explorer10 system, HisTrap FF and HiPrep Sephacryl S-300 16/60 columns from GE HEALTHCARE were used.

Further materials, instrumentations and suppliers are indicated in the subsequent chapters.

\subsection{Media and growth conditions}

Chemicals for media preparation were dissolved in deionized $\mathrm{H}_{2} \mathrm{O}\left(\mathrm{dH}_{2} \mathrm{O}\right)$ and sterilized by autoclavation at $121^{\circ} \mathrm{C}$ for $20 \mathrm{~min}$ at two bar. Thermally unstable supplementations were dissolved in $\mathrm{dH} 2 \mathrm{O}$ and sterile filtered.

\subsubsection{Bacterial growth}

Escherichia coli strain DH5a ${ }^{\mathrm{TM}}$ (Hanahan, 1985), were used for construction and amplification of recombinant plasmids. Rosetta II E. coli strain (NOVAGEN ${ }^{\circledR}$, MERCK) was used for the recombinant expression of $A$. nidulans proteins. Genotypes of these strains are given in TABLE 1. E. coli strains were cultivated in lysogeny broth (LB) (Bertani, 1951) medium (1\% (w/v) tryptophan, $0.5 \%(\mathrm{w} / \mathrm{v})$ yeast extract, $1 \%(\mathrm{w} / \mathrm{v}) \mathrm{NaCl})$ on a rotary shaker at $37^{\circ} \mathrm{C}$. Ampicillin was used as selective agent in a concentration of $100 \mathrm{mg} / \mathrm{mL}$. Kanamycin was used in a concentration of $50 \mathrm{mg} / \mathrm{mL}$ and chloramphenicol in a concentration of $34 \mathrm{mg} / \mathrm{mL}$. Solid medium was prepared by supplementation with $2 \%(\mathrm{w} / \mathrm{v})$ agar. 
TABLE 1: $E$. coli strains used in this study.

\begin{tabular}{ll}
\hline Strain name & Genotype \\
\hline DH5a ${ }^{\mathrm{TM}}$ & $\mathrm{F}-, \Delta(\operatorname{argF-lac}) 169, \varphi 80 \mathrm{~d} / \mathrm{acZ} \Delta \mathrm{M} 15, \Delta p h o A 8$, glnX44(AS), $\lambda-$, deoR481, \\
rfbC1, gyrA96(NalR), recA1, endA1, thiE1, sdR17
\end{tabular}

\subsubsection{Fungal growth}

The $A$. nidulans ve $A^{+}$strain AGB 551 (Bayram et al., 2012) was used as wildtype (wt) host for A. nidulans strains constructed in this study. A. nidulans strains were grown in minimal medium (MM) (1\% (w/v) glucose, $7 \mathrm{mM} \mathrm{KCl,} 2 \mathrm{mM} \mathrm{MgSO}_{4}, 70 \mathrm{mM} \mathrm{NaNO}_{3}, 11.2 \mathrm{mM} \mathrm{KH}_{2} \mathrm{PO}_{4}, 0.1 \%$ (v/v) trace element solution $\mathrm{pH} 5.5$ (Käfer, 1977)). 2\% (w/v) agar was added for solid MM plates. Supplements such as $0.1 \%$ pyridoxine-HCl (SIGMA-ALDRICH), 0.1\% uridine (ROTH), 5\% uracil (ROTH), added in appropriate amounts. Aspergilli were grown at $37^{\circ} \mathrm{C}$ in baffled flasks under shaking conditions on a rotary shaker for vegetative growth for 24 hours. $A$. nidulans strains were grown on solid MM plates under constant white light for three to seven days, for the induction asexual development. The promotion of sexual development was achieved by growing fungal strains in the dark for three to seven days on solid MM plates, which were tightly sealed with Parafilm ${ }^{\circledR}$ M (MERCK) to prevent oxygen supply. Conidiospores were harvested in $0.96 \%(\mathrm{w} / \mathrm{v}) \mathrm{NaCl}$ with $0.002 \%(\mathrm{v} / \mathrm{v})$ Tween-80 (SIGMA-ALDRICH) and stored at $4^{\circ} \mathrm{C}$. All $A$. nidulans strains used in this study are listed in TABLE 2.

TABLE 2: Fungal strains used in this study.

$\mathrm{P}=$ promoter, phleomycin resistance (non-recyclable), p.c. $=$ personal communication

\begin{tabular}{lll}
\hline Strain & Genotype & Reference \\
\hline FGSC A4 & veA & (McCluskey et al., \\
AGB551 & $n k u A \Delta:: \operatorname{argB}$, pyrG89, pyroA4, veA
\end{tabular}




\begin{tabular}{|c|c|c|}
\hline AGB808 & 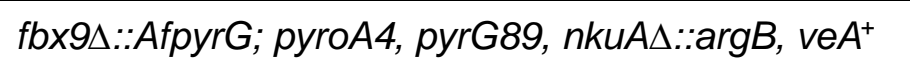 & Ö. Bayram, p.c. \\
\hline AGB809 & fbx10 $\Delta::$ AfpyrG; pyroA4, pyrG89, nkuA $\Delta:: \arg B, v e A^{+}$ & Ö. Bayram, p.c. \\
\hline AGB810 & 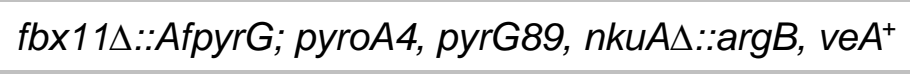 & Ö. Bayram, p.c. \\
\hline AGB811 & 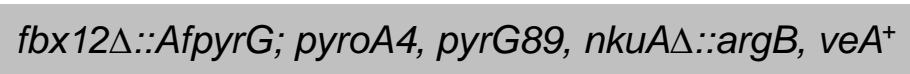 & Ö. Bayram, p.c. \\
\hline AGB812 & 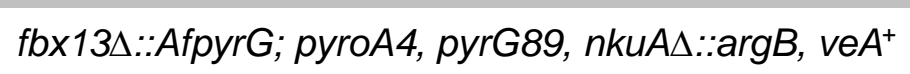 & Ö. Bayram, p.c. \\
\hline AGB813 & 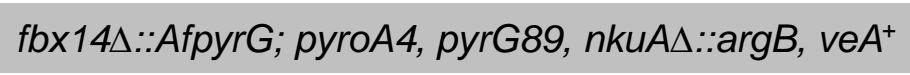 & Ö. Bayram, p.c. \\
\hline AGB814 & 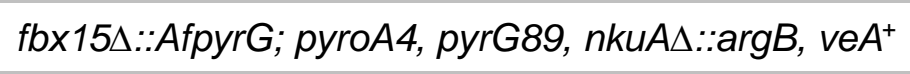 & Ö. Bayram, p.c. \\
\hline AGB815 & 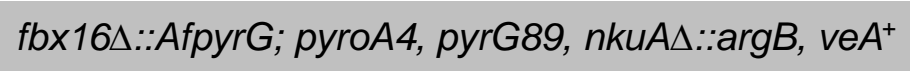 & Ö. Bayram, p.c. \\
\hline AGB816 & 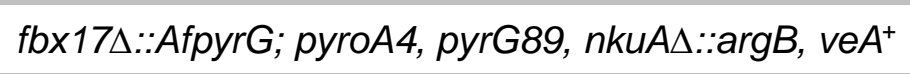 & Ö. Bayram, p.c. \\
\hline AGB817 & 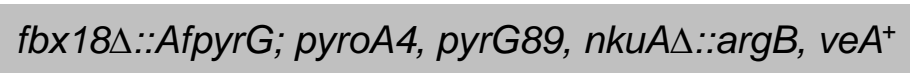 & Ö. Bayram, p.c. \\
\hline AGB818 & 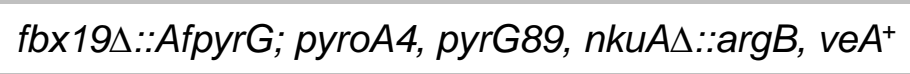 & Ö. Bayram, p.c. \\
\hline AGB819 & 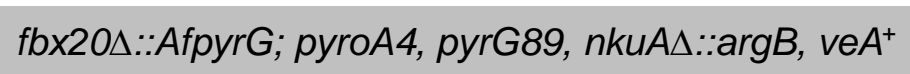 & Ö. Bayram, p.c. \\
\hline AGB820 & 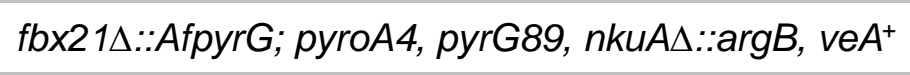 & Ö. Bayram, p.c. \\
\hline AGB821 & fbx22 $\Delta:: A f p y r G ;$ pyroA4, pyrG89, nkuA $\Delta:: \arg B, v e A^{+}$ & Ö. Bayram, p.c. \\
\hline AGB822 & 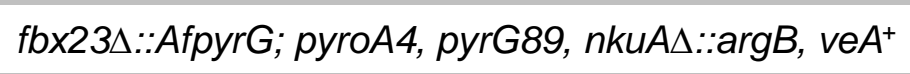 & Ö. Bayram, p.c. \\
\hline AGB823 & 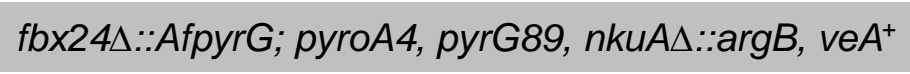 & Ö. Bayram, p.c. \\
\hline AGB824 & 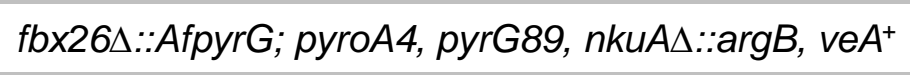 & Ö. Bayram, p.c. \\
\hline AGB825 & 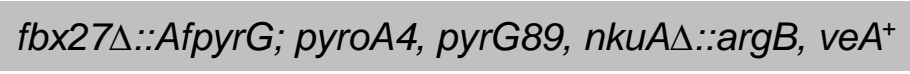 & Ö. Bayram, p.c. \\
\hline AGB826 & 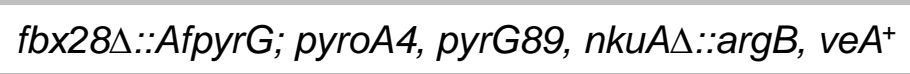 & Ö. Bayram, p.c. \\
\hline AGB827 & 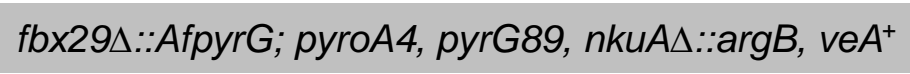 & Ö. Bayram, p.c. \\
\hline AGB828 & 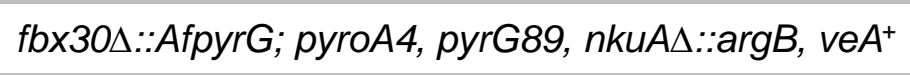 & Ö. Bayram, p.c. \\
\hline AGB829 & 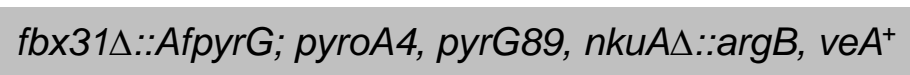 & Ö. Bayram, p.c. \\
\hline AGB830 & 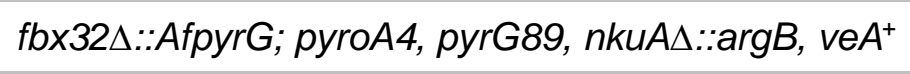 & Ö. Bayram, p.c. \\
\hline AGB831 & 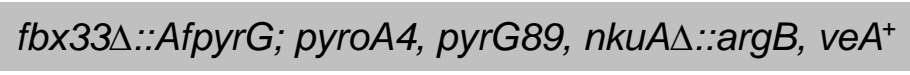 & Ö. Bayram, p.c. \\
\hline AGB832 & 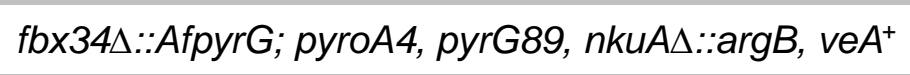 & Ö. Bayram, p.c. \\
\hline AGB833 & 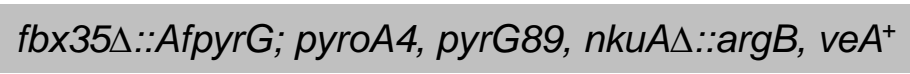 & Ö. Bayram, p.c. \\
\hline AGB834 & 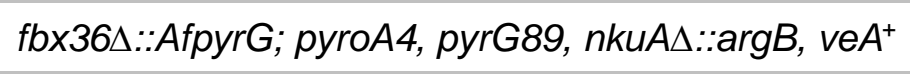 & Ö. Bayram, p.c. \\
\hline AGB835 & 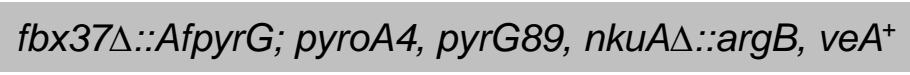 & Ö. Bayram, p.c. \\
\hline AGB836 & 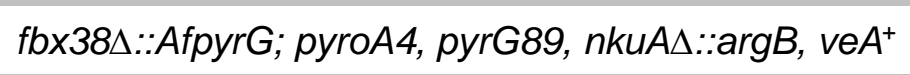 & Ö. Bayram, p.c. \\
\hline AGB837 & 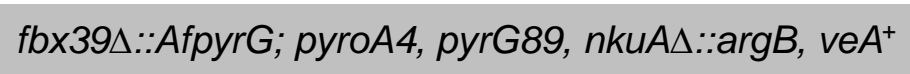 & Ö. Bayram, p.c. \\
\hline AGB838 & 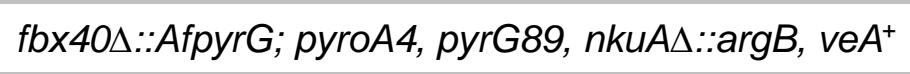 & Ö. Bayram, p.c. \\
\hline AGB839 & 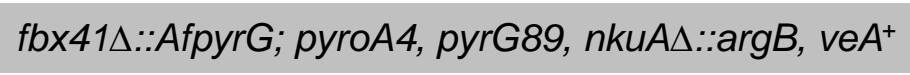 & Ö. Bayram, p.c. \\
\hline AGB840 & 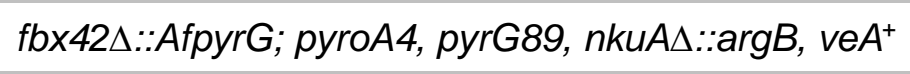 & Ö. Bayram, p.c. \\
\hline AGB841 & 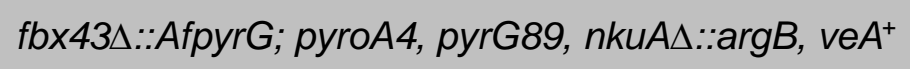 & Ö. Bayram, p.c. \\
\hline AGB842 & 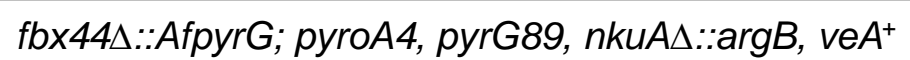 & Ö. Bayram, p.c. \\
\hline
\end{tabular}




\begin{tabular}{|c|c|c|}
\hline AGB843 & 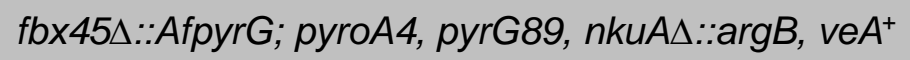 & Ö. Bayram, p.c. \\
\hline AGB844 & 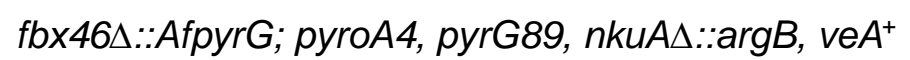 & Ö. Bayram, p.c. \\
\hline AGB845 & 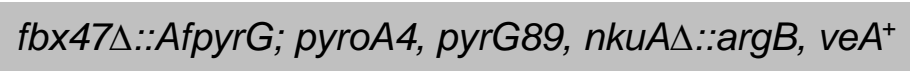 & Ö. Bayram, p.c. \\
\hline AGB846 & 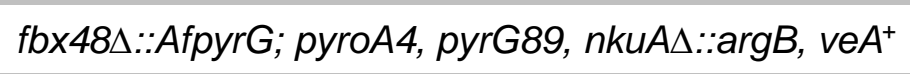 & Ö. Bayram, p.c. \\
\hline AGB847 & 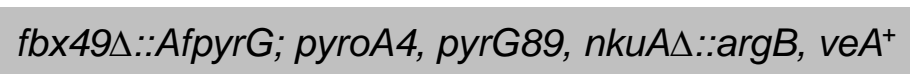 & Ö. Bayram, p.c. \\
\hline AGB848 & 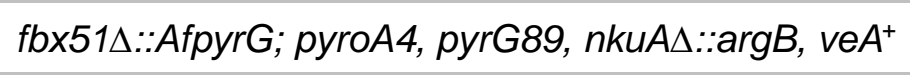 & Ö. Bayram, p.c. \\
\hline AGB849 & 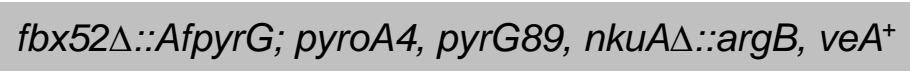 & Ö. Bayram, p.c. \\
\hline AGB850 & 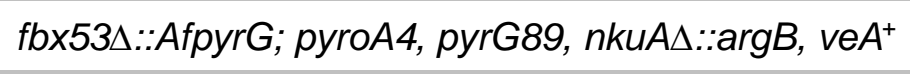 & Ö. Bayram, p.c. \\
\hline AGB851 & 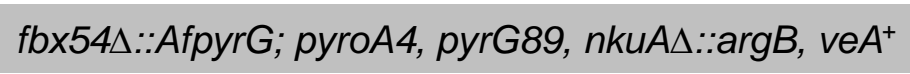 & Ö. Bayram, p.c. \\
\hline AGB852 & 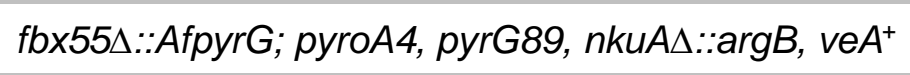 & Ö. Bayram, p.c. \\
\hline AGB853 & 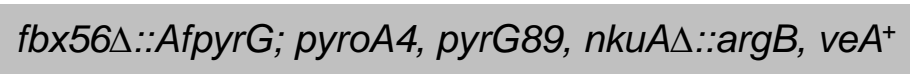 & Ö. Bayram, p.c. \\
\hline AGB854 & 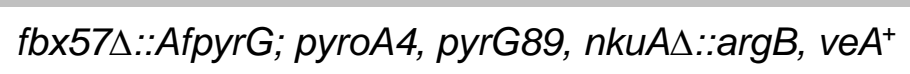 & Ö. Bayram, p.c. \\
\hline AGB855 & 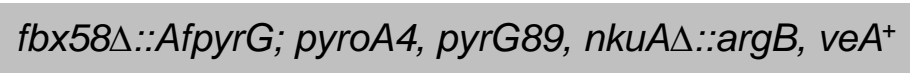 & Ö. Bayram, p.c. \\
\hline AGB856 & 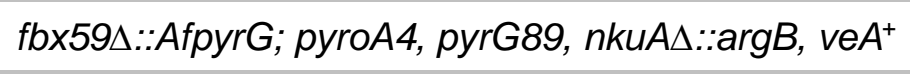 & Ö. Bayram, p.c. \\
\hline AGB857 & 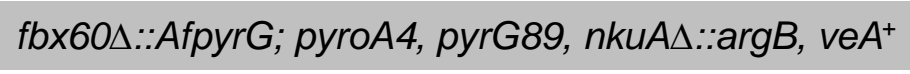 & Ö. Bayram, p.c. \\
\hline AGB858 & 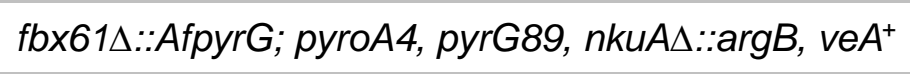 & Ö. Bayram, p.c. \\
\hline AGB859 & 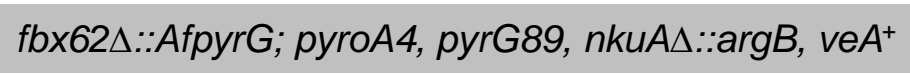 & Ö. Bayram, p.c. \\
\hline AGB860 & 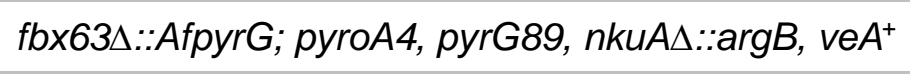 & Ö. Bayram, p.c. \\
\hline AGB861 & 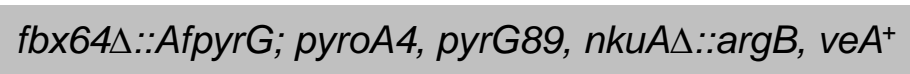 & Ö. Bayram, p.c. \\
\hline AGB862 & 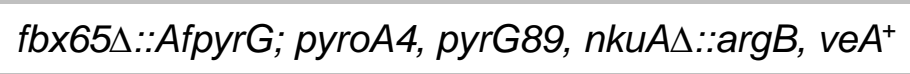 & Ö. Bayram, p.c. \\
\hline AGB863 & 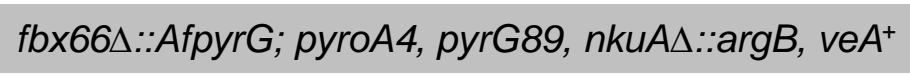 & Ö. Bayram, p.c. \\
\hline AGB864 & 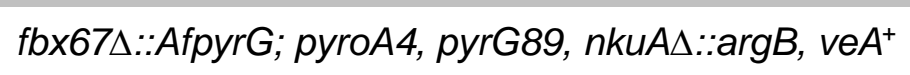 & Ö. Bayram, p.c. \\
\hline AGB865 & 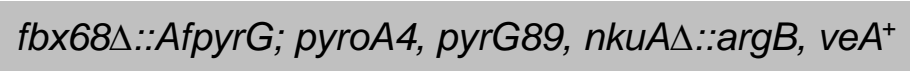 & Ö. Bayram, p.c. \\
\hline AGB866 & 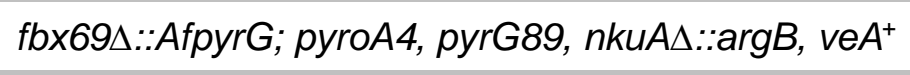 & Ö. Bayram, p.c. \\
\hline AGB867 & 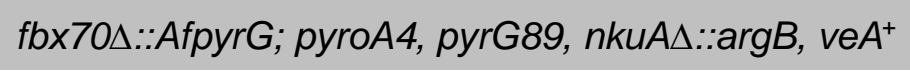 & Ö. Bayram, p.c. \\
\hline AGB868 & 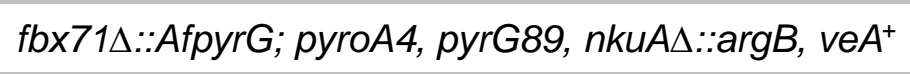 & Ö. Bayram, p.c. \\
\hline AGB869 & 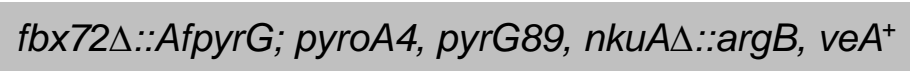 & Ö. Bayram, p.c. \\
\hline AGB870 & 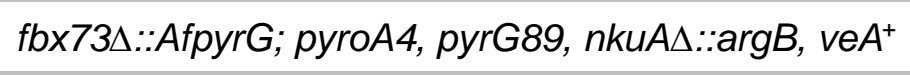 & Ö. Bayram, p.c. \\
\hline AGB871 & 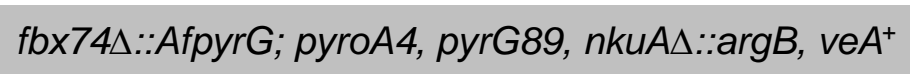 & Ö. Bayram, p.c. \\
\hline AGB1057 & nkuA $\triangle:$ argB, pyroA4, pyrG89, ve $A^{+}, \operatorname{vos} A \Delta:: s i x$ & This study \\
\hline AGB1062 & 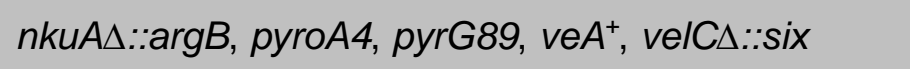 & K. Thieme, p.c. \\
\hline AGB1064 & 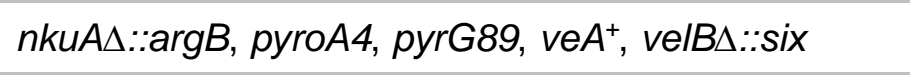 & This study \\
\hline AGB1066 & nkuA $\triangle:: a r g B$, pyroA4, pyrG89, veA $A^{+}$veA $\Delta:: s i x$ & J. Gerke, p.c. \\
\hline AGB1131 & $n k u A \Delta:: a r g B$, pyroA4, pyrG89, ve $A^{+}, \operatorname{vel}^{I D D_{\Delta}}: . \mathrm{six}$ & This study \\
\hline AGB1132 & nkuA $\triangle:: a r g B$, pyroA4, pyrG89, ve $A^{+}$, velB::sgfp::six & This study \\
\hline
\end{tabular}




\begin{tabular}{|c|c|c|}
\hline AGB1133 & 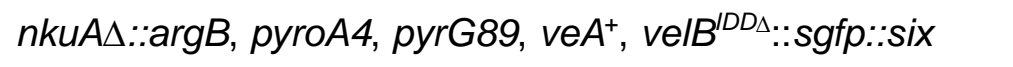 & This study \\
\hline AGB1134 & $n k u A \Delta:: \arg B$, pyroA4, pyrG89, veA $A^{+}$, velB $B^{A . f \_I D D .:: s i x}$ & This study \\
\hline AGB1135 & 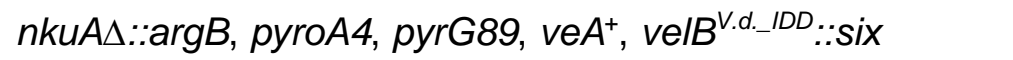 & This study \\
\hline AGB1136 & $\begin{array}{l}\text { nkuAA::argB, pyroA4, pyrG89, veA } A^{+} \text {, velB::sgfp::six, } \\
\text { vosA::ha::six }\end{array}$ & This study \\
\hline AGB1137 & $\begin{array}{l}\text { nkuA } \Delta:: \text { argB, pyroA4, pyrG89, ve } A^{+}, \text {vel} B^{I D D_{\Delta}:: s g f p:: s i x,} \\
\text { vosA::ha::six }\end{array}$ & This study \\
\hline AGB1138 & nkuA $\Delta:: \arg B$, pyroA4, pyrG89, veA $A^{+}$, velC:sgfp:six & This study \\
\hline AGB1139 & 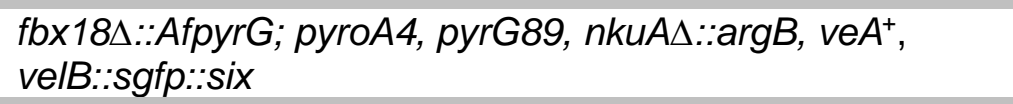 & This study \\
\hline AGB1140 & $\begin{array}{l}\text { nkuAA::argB, pyroA4, pyrG89, ve } A^{+}, \text {veA } \triangle:: \text { six, } \\
\text { velB } B^{I D D_{\Delta}}:: \text { six }\end{array}$ & This study \\
\hline AGB1141 & $\begin{array}{l}\text { nkuA } \Delta:: \text { argB }, \text { pyroA4, pyrG89, ve } A^{+}, \text {velC } \Delta .: \text { six, } \\
\text { velB } B^{I D D_{\Delta}:: \text { six }}\end{array}$ & This study \\
\hline AGB1142 & 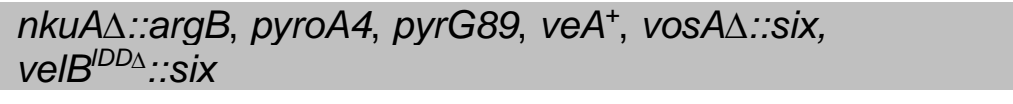 & This study \\
\hline AGB1143 & 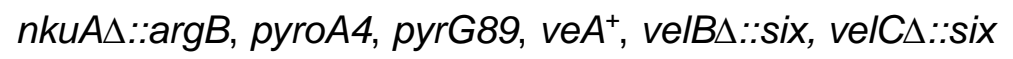 & This study \\
\hline AGB1144 & nkuA $\Delta:: a r g B$, pyroA4, pyrG89, ve $A^{+}$, velB $\Delta::$ six, ve $A \Delta:: s i x$ & This study \\
\hline AGB1145 & 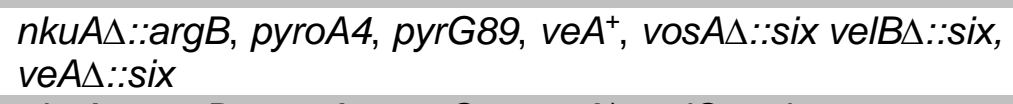 & This study \\
\hline AGB1146 & 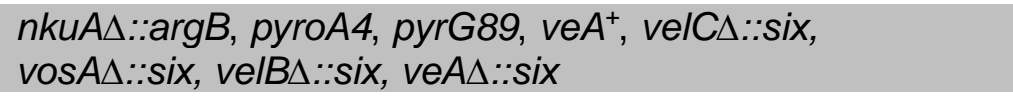 & This study \\
\hline AGB1148 & $\begin{array}{l}\text { nkuA } \Delta:: \text { arg } B, \text { pyroA4, pyrG89, veA+, } \\
\text { velB } B^{I D \Delta}:: \text { velB::sgfp:six }\end{array}$ & This study \\
\hline
\end{tabular}

\subsection{Morphological methods}

\subsubsection{Phenotypic analyses of fungal strains}

For phenotypic analyses either 2000 spores were spotted in the middle of the agar plate or $10^{5}$ spores were distributed over the whole plate. Agar plates were incubated for three or seven days under either asexual or sexual development promoting conditions. Photomicrographic pictures of $A$. nidulans colonies were obtained by utilization of an Axiolab microscope (CARL ZEISS MICROSCOPY GMBH, Jena, Germany) or with the help of a binocular microscope SZX12ILLB2-200 (OLYMPUS DEUTSCHLAND GMBH, Hamburg, Germany). Visualization was performed with an OLYMPUS SC30 digital camera and pictures were processed with the cellSens software (Olympus DEUTSCHLAND GMBH, Hamburg, Germany).

\subsubsection{Conidiospore quantification}

Conidiospore numbers were determined by utilization of Coulter Z2 particle counter (BECKMAN COULTER GMBH, Krefeld, Germany) or with a Thoma cell counting chamber (hemocytometer) (PAUl MARIENFELd GmbH AND Co. KG, Lauda-Königshofen, Germany). The determination of total conidiospore numbers was accomplished by complete harvesting of all conidiospore from 
agar plates with a prior inoculum of $10^{5}$ spores in $0.96 \%(w / v) ~ N a C l$ with $0.002 \%(\mathrm{v} / \mathrm{v})$ Tween80

\subsection{Nucleic acid methods}

\subsubsection{Isolation and purification of plasmid-DNA and linearized DNA fragments}

Extraction of plasmid-DNA from E. coli cultures was performed by employment of the QIAprep ${ }^{\circledR}$ Spin Miniprep Kit (QIAGEN) or the NucleoSpin ${ }^{\circledR}$ Plasmid Kit (MACHEREY-NAGEL) according to manufacturer's specifications. The elution of plasmid-DNA from spin columns was done with $\mathrm{dH}_{2} \mathrm{O}$ and stored at $-20^{\circ} \mathrm{C}$.

10x DNA loading dye (10\% (v/v) Ficoll 400, 200 mM EDTA pH 8.0, 0.2\% (w/v) bromophenol blue, $0.2 \%(\mathrm{w} / \mathrm{v})$ xylene cyanol $\mathrm{FF})$ was added to linearized DNA fragments of PCR amplifications and enzymatic digests for linearization of plasmids and construct excision and separated by agarose gel electrophoresis. Gel pieces including desired DNA bands were cut out of the gel and DNA was purified by utilization of the QIAquick $^{\circledR}$ Gel Extraction Kit (QIAGEN) or the NucleoSpin ${ }^{\circledR}$ Gel and PCR Clean-up Kit from MACHEREY-NAGEL GMBH \& Co. KG (Düren, Germany).

\subsubsection{Polymerase chain reaction (PCR)}

Amplification of DNA fragments for plasmid construction and determination of the presence of desired plasmids in E. coli after plasmid construction via ligation or seamless cloning (colony PCR) (Bergkessel and Guthrie, 2013; Hofmann and Brian, 1991) was achieved by employing polymerase chain reaction (PCR) (Saiki et al., 1988). PCRs were performed in T Professional Standard 96, T Professional Trio 48 and T Professional Standard 96 Gradient thermocyclers from BIOMETRA GMBH (Göttingen, Germany) and in Primus 96 Thermal Cyclers from MWG BIOTECH AG (Ebersberg, Germany). For DNA amplification via PCR, Phusion ${ }^{\circledR}$ High-Fidelity DNA Polymerase (THERMO FISHER SCIENTIFIC) was used. PCR programs were created after manufacturer's instructions and in accordance with calculated melting temperatures of employed primers. The OligoCalc program was used for calculation of primer $\mathrm{T}_{M}$ and salt adjusted temperatures were regarded as best assumption (Kibbe, 2007). 


\subsubsection{Agarose gel electrophoresis}

The separation of DNA fragments according to their size was achieved by the employment of agarose gel electrophoresis (Lee et al., 2012). 1\% (w/v) agarose gels containing $0.001 \mathrm{mg} / \mathrm{mL}$ ethidium bromide were used in TAE buffer (40 mM Tris, $20 \mathrm{mM}$ acetic acid, $1 \mathrm{mM}$ EDTA). DNA was mixed with 10x loading dye and visualized in-gel by exposure to UV light $(\lambda=254 \mathrm{~nm})$ in a Gel iX20 Imager Windows Version and the Intas GDS gel documentation software from INTAS ScIENCE IMAGING INSTRUMENTS GmBH (Göttingen, Germany) or on a TFX-20 MX Vilber Lourmat Super Bright transilluminator (SIGMA-ALDRICH).

\subsubsection{Quantitative real-time polymerase chain reaction}

Quantitative real-time $\underline{\mathrm{PCR}}$ ( $\mathrm{qRT}-\mathrm{PCR}$ ) was employed for the measurement of gene expression. Therefore, the MESA GREEN qPCR MasterMix Plus for SYBR ${ }^{\circledR}$ Assay purchased from EUROGENTEC (Lüttich, Belgium) in a CFX ConnectTM Real-Time System (BIORAD) was used. The qRT-PCR protocol, which was used, is given in TABLE 3. The Primer3 software was employed for primer design (Koressaar and Remm, 2007; Untergasser et al., 2012) and primers are given in TABLE 4. Gene expression was measured from 1:5 dilutions of respective cDNA. Obtained qRT-PCR data was analyzed with the CFX Manager ${ }^{\mathrm{TM}} 3.1$ software package (BIO-RAD) using the 2- $\triangle \triangle C T$ method for relative quantification of gene expression (Schmittgen and Livak, 2008). Expression of $h 2 A$ (AN3468) and $15 S$ rRNA were used for $A$. nidulans qRTPCR as references for relative quantification. qRT-PCR experiments were conducted in several biological replicates as indicated. Each biological replicate was measured in three technical replicates.

TABLE 3: qRT-PCR protocol used in this study. Steps 2 to 4 were repeated for 36 times.

\begin{tabular}{lll|l}
\hline Step & Temperature $\left[\mathbf{C}^{\circ}\right]$ & Duration [min] \\
\hline 1 & 95 & $5: 00$ & \\
2 & 95 & $0: 15$ & $36 \times$ \\
3 & 60 & $0: 22$ & \\
4 & 72 & $0: 40$ & \\
5 & 95 & $0: 10$ & \\
6 & Melt curve: 65 to 95, & $0: 05$ & \\
\hline
\end{tabular}


TABLE 4: Primers used for qRT-PCR in this study.

\begin{tabular}{llll}
\hline Designation & Gene & $\mathbf{5}^{\prime}$ - sequence - 3' & Size \\
\hline jg816 & velB A & CCC CTC CGT GTA TCC GTC TAA T & $22 \mathrm{mer}$ \\
jg817 & velB B & AGC CGA GTG CTT CAC AAG ATT T & $22 \mathrm{mer}$ \\
kt278 & 15S rRNA A & GAT CCG CGA AAA ACC TTA CCA C & $22 \mathrm{mer}$ \\
kt279 & 15S rRNA B & TGG CAC GTC TAT AGC CCA CAG T & 22mer \\
kt312 & h2A.X A & TCT CGA GCT TGC TGG AAA CG & 20mer \\
kt313 & h2A.X B & CAC CCT GGG CAA TAG TGA CG & 20mer \\
\hline
\end{tabular}

\subsubsection{Isolation and purification of fungal genomic DNA}

A. nidulans strains were grown overnight $(\mathrm{o} / \mathrm{n})$ in submerged cultures for the extractions of genomic DNA (gDNA). Vegetative mycelia were harvested through Miracloth filters (MERCK), frozen in liquid nitrogen and ground with a MM400 Table mill from RETSCH TECHNOLOGY GMBH (Haan, Germany). $500 \mu \mathrm{L}$ genomic DNA lysis buffer (Lee and Taylor, 1990) (50 mM Tris$\mathrm{HCl} \mathrm{pH} \mathrm{7.2,} 50 \mathrm{mM}$ EDTA, 3\% (w/v) SDS, 1\% (v/v) $\beta$-mercaptoethanol) was added to the ground mycelia and incubated for $15 \mathrm{~min}$ at $65^{\circ} \mathrm{C}$. Afterwards this mix was supplied with $100 \mu \mathrm{L} 8 \mathrm{M}$ potassium acetate, inverted and centrifuged for $15 \mathrm{~min}$ at $13000 \mathrm{rpm}$ at room temperature (rt). This step was repeated. Subsequently, the supernatant was transferred into new reaction tubes, mixed with $300 \mu \mathrm{L}$ isopropanol and centrifuged for 10 min at $13000 \mathrm{rpm}$ at $\mathrm{rt}$. The DNA pellets were washed with $70 \%(\mathrm{v} / \mathrm{v})$ ethanol and dried at $42^{\circ} \mathrm{C}$. Finally, the DNA was resolved in $\mathrm{H}_{2} \mathrm{O}$ at $65^{\circ} \mathrm{C}$.

\subsubsection{Isolation and purification of fungal RNA}

A. nidulans strains were grown asexually for RNA isolation. After harvesting through sterile Miracloth filters, mycelia were frozen in liquid nitrogen and ground with a table mill (RETSCH) directly before RNA isolation. The RNeasy ${ }^{\circledR}$ Plant Miniprep Kit from QIAGEN (Hilden, Germany) was employed for isolation of approximately $200 \mu \mathrm{L}$ ground mycelia according to manufacturer's instructions without addition of $\beta$-mercaptoethanol. Concentrations were measured with a Nanodrop ND-1000 (PEQLAB). cDNA was transcribed from $0.8 \mu$ R RNA with the QuantiTect ${ }^{\circledR}$ Reverse Transcription Kit (QIAGEN) according to manufacturer's conditions. 


\subsection{Plasmid construction for genetic manipulation of $\boldsymbol{A}$. nidulans}

\subsubsection{Cloning strategies}

DNA sequences were fused for plasmid construction by fusion-PCR (Szewczyk et al., 2006). Furthermore, the GeneArt ${ }^{\circledR}$ Seamless Cloning and Assembly Kit (INVITROGEN) or the GeneArt ${ }^{\circledR}$ Seamless Cloning and Assembly Enzyme Mix (INVITROGEN) were used. DNA amplification of DNA fragment was conducted from $A$. nidulans FGSC A4 gDNA as template. The constructs, which harbor a recyclable marker cassette, were cloned into the EcoRV multiple cloning site of pBluescript SK+. The excision of the genetic constructs, which were constructed this way, was ensured by introducing Mssl restriction sites. This way constructs were linearized and integrated on-locus into the genome of transformed $A$. nidulans.

\subsubsection{Primer and plasmid design}

The plasmids, which were generated and used in this study, are listed in TABLE 5 and TABLE 6 contains the primers, which were used for plasmid construction. Primers and genetic construct maps were designed with the Lasergene software package from DNA STAR INC. (Madison, WI, USA). Genetic information was accessed from AspGD (Cerqueira et al., 2014) and CADRE (Mabey Gilsenan et al., 2012). The next chapter comprises detailed descriptions of the construction strategies. For almost all plasmids containing constructs excised with Mssl (in GTTT/AAAC pattern) for transformation in $A$. nidulans, a quadruplet naturally comprising a GTTT or AAAC sequence was used as terminal sequence for the plasmid backbone. The respective terminal primers of these constructs introduce the corresponding second half of the Mssl restriction site. This has the advantage that no additional base pairs were transformed into the $A$. nidulans genome and genes, which might lie in respective regions, were not interrupted. Primers used for seamless cloning reactions were created to insert 15 base pairs (bp) complementary to adjoining sequences in the way that two bordering sequences share a 15 bp homology region. 
TABLE 5: Plasmids constructed and used in this study.

pBluescript SK+ was used as backbone for all plasmids constructed in this study, if not stated otherwise. ${ }^{\mathrm{P}}=$ promoter, ${ }^{\mathrm{t}}=$ terminator, ${ }^{\mathrm{R}}=$ resistance, natRM $=$ recyclable nat $^{\mathrm{R}}$ resistance cassette from pME4304, phleoRM = recyclable phleo ${ }^{R}$ resistance cassette from pME4305, p.c. = personal communication.

\begin{tabular}{|c|c|c|}
\hline Plasmid & Description & Reference \\
\hline pME4319 & $\begin{array}{l}\text { six-PxylP::B-rec:::trpC } C^{t}-p h l e o^{R}-\text { six in EcoRV of } \\
\text { pBluescript SK+ with Eco72l and Swal restriction } \\
\text { sites }\end{array}$ & J. Gerke, p.c. \\
\hline pETM13-VosA190S & $\begin{array}{l}\text { vosA1-190 from cDNA, with a 3' coding } \\
\text { sequence for a Strep-tag, cloned in pETM-13 } \\
\text { digested with Ncol }\end{array}$ & Ahmed et al., 2013 \\
\hline pME3815 & $\begin{array}{l}\text { velB from cDNA, cloned in pETM-13 digested } \\
\text { with } \mathrm{Ncol} / \text { Xhol }\end{array}$ & Ahmed et al., 2013 \\
\hline pME4292 & Plasmid contains sgfp & Jöhnk et al., 2016 \\
\hline pME4304 & $\begin{array}{l}\text { six-PxylP::ß-rec::trpC } C^{t} \text {-nat }{ }^{\mathrm{R}} \text {-six with Sfil restriction } \\
\text { sites }\end{array}$ & J. Gerke, p.c. \\
\hline pME4305 & $\begin{array}{l}\text { six-PxylP::B-rec:::trpC } C^{t}-p h l e o^{\mathrm{R}} \text {-six with Sfil } \\
\text { restriction sites }\end{array}$ & J. Gerke, p.c. \\
\hline pME4574 & veA $A:$ natRM & J. Gerke, p.c. \\
\hline pME4602 & velC $\Delta:$ :phleoRM & K. Thieme, p.c. \\
\hline pME4603 & vosA $\triangle:$ :natRM & This study \\
\hline pME4604 & velB $\Delta::$ natRM & This study \\
\hline pME4686 & velB $B^{I D D_{\Delta}}:$ phleoRM & This study \\
\hline pME4687 & velB::sgfp::natRM & This study \\
\hline pME4688 & velB ${ }^{I D D_{\Delta}}: .: s g f p:$ :phleoRM & This study \\
\hline pME4689 & 3' flanking region of velB in Eco72l of pME4319 & This study \\
\hline pME4690 & velB $B^{A . f . I D D}:$ phleoRM & This study \\
\hline pME4691 & velB $B^{V . d . \_I D D}:$. phleoRM & This study \\
\hline pME4692 & $\begin{array}{l}\text { velB }{ }^{I D D_{\Delta}} \text { from pME3815, cloned in pETM-13 } \\
\text { digested with } N \text { col/ } \text { Xhol }\end{array}$ & This study \\
\hline pME4693 & vosA::ha::phleoRM & This study \\
\hline pME4694 & velC::sgfp::natRM & This study \\
\hline
\end{tabular}

TABLE 6: Oligonucleotides used for amplifications, plasmid constructions and sequencing.

\begin{tabular}{llllll}
\hline Name & \multicolumn{2}{l}{$\mathbf{5}$-sequence - $\mathbf{3}$} & & Size \\
\hline JG45 & CCC CAT GGC GAT GTA & CGC & TGT & TGA GGA TAG G & 31mer \\
JG46 & CCC TCG AGG TAT TCG TTA TCC AGA CCA TC & 29mer \\
JG466 & TCT GAT ATC TAT AGG TCA ATA GAG & 24mer \\
\hline
\end{tabular}




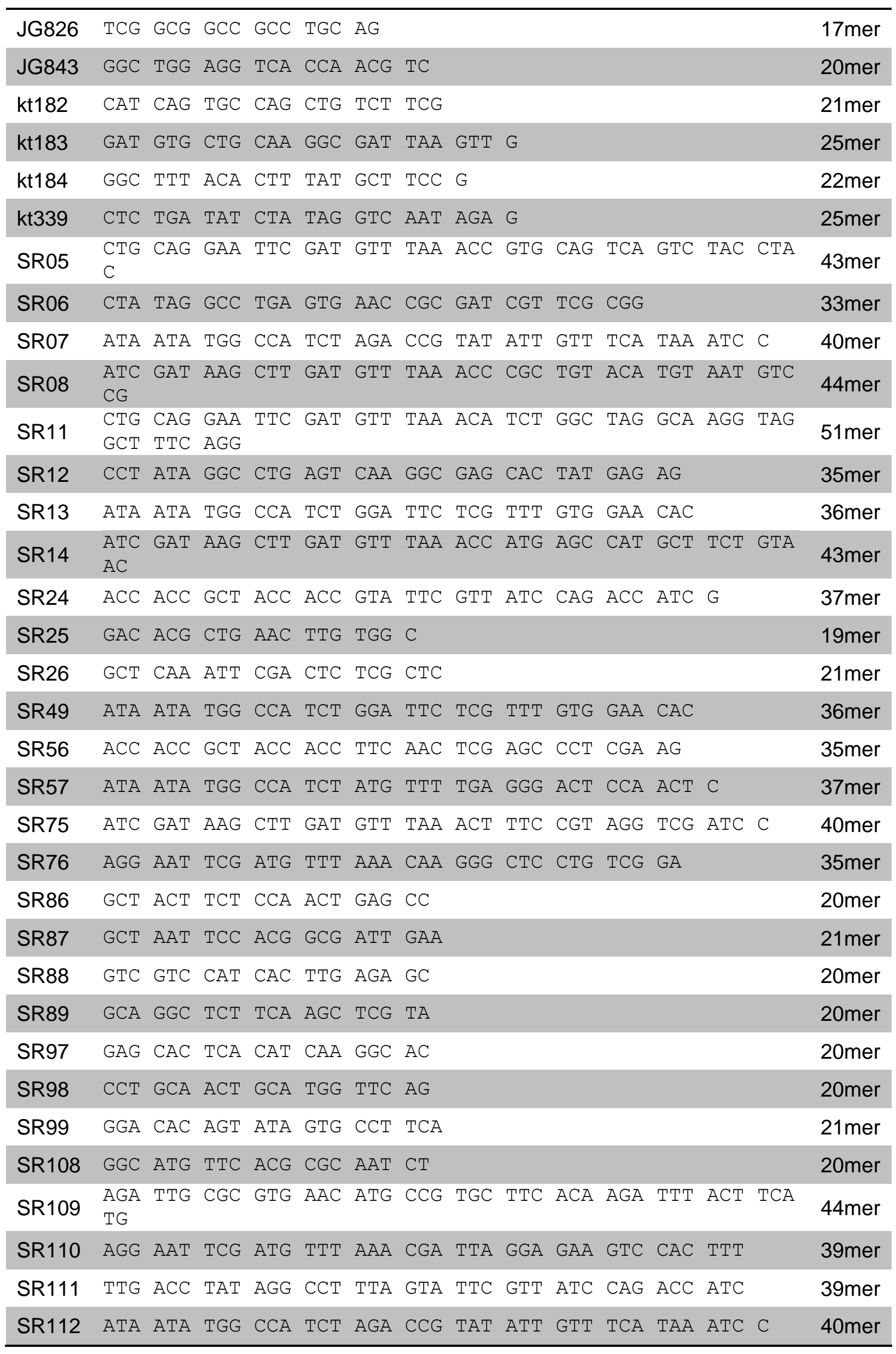




\begin{tabular}{|c|c|c|c|c|c|c|c|c|c|c|c|c|c|c|c|}
\hline SR201 & $\begin{array}{l}\text { CTA } \\
\text { GTA }\end{array}$ & $\begin{array}{l}\text { TAG } \\
\text { CCG }\end{array}$ & $\begin{array}{l}\text { GCC } \\
\text { AGG }\end{array}$ & $\begin{array}{l}\text { TGA } \\
\text { AGT }\end{array}$ & $\begin{array}{l}\text { GTG } \\
\text { TCC }\end{array}$ & $\begin{array}{l}\text { TTA } \\
\text { GTT }\end{array}$ & $\begin{array}{l}\text { GGC } \\
\text { CGC }\end{array}$ & GTA & GTC & GGG & GAC & GTC & GTA & GGG & 63 mer \\
\hline SR253 & GTG & CTT & CAC & AAG & ATT & TAC & TTC & ATG & & & & & & & $24 \mathrm{mer}$ \\
\hline SR254 & AAG & TAA & ATC & TTG & TGA & AGC & ACC & AGC & GCG & AGG & TGA & $\mathrm{ACC}$ & $\mathrm{TC}$ & & $38 \mathrm{mer}$ \\
\hline SR266 & $\begin{array}{l}\text { AAG } \\
\mathrm{C}\end{array}$ & TAA & ATC & TTG & TGA & AGC & $\mathrm{ACT}$ & CAG & CCA & CGT & CGC & CAT & CAA & TAT & $43 \mathrm{mer}$ \\
\hline SR267 & AGA & TTG & CGC & GTG & $A A C$ & ATG & $\mathrm{CCA}$ & CCG & GGC & $\mathrm{CCC}$ & GCC & GGA & A & & $37 \mathrm{mer}$ \\
\hline
\end{tabular}

\subsubsection{Recyclable marker cassettes as selection markers}

All plasmids, which were built in this study and used for homologous recombination, contain recyclable marker cassettes. They are based on the bacterial recombination system, which utilizes a prokaryotic small $\beta$-serine recombinase and its six recognition sequence (Canosa et al., 1996; Hartmann et al., 2010; Rojo et al., 1993; Rojo and Alonso, 1994). Thereby, the excision of the respective marker cassette of the fungal genome is allowed after successful transformation (FIGURE 12). The utilization of this system enables to employ marker-free mutations and only leaves a small six recognition site of $100 \mathrm{bp}$. This allows the employment of several mutations in one background strain with the same selection marker. The integration of large resistance cassettes is avoided and thereby its putative interference with the genome of the host strain is prevented. In the presented study, two different recyclable marker cassettes were used. pME4304 (J. Gerke, p.c.) contains the nat1 gene from Streptomyces noursei, which is resistant against nourseothricin (Kück and Hoff, 2006). The nourseothricin resistance marker cassette from pME4304 is termed natRM hereafter. pME4305 (J. Gerke, p.c.) contains the ble gene from Streptoalloteichus hindustanus, which therefore is resistant to phleomycin (Drocourt et al., 1990). The phleomycin resistance marker cassette is termed phleoRM hereafter. 


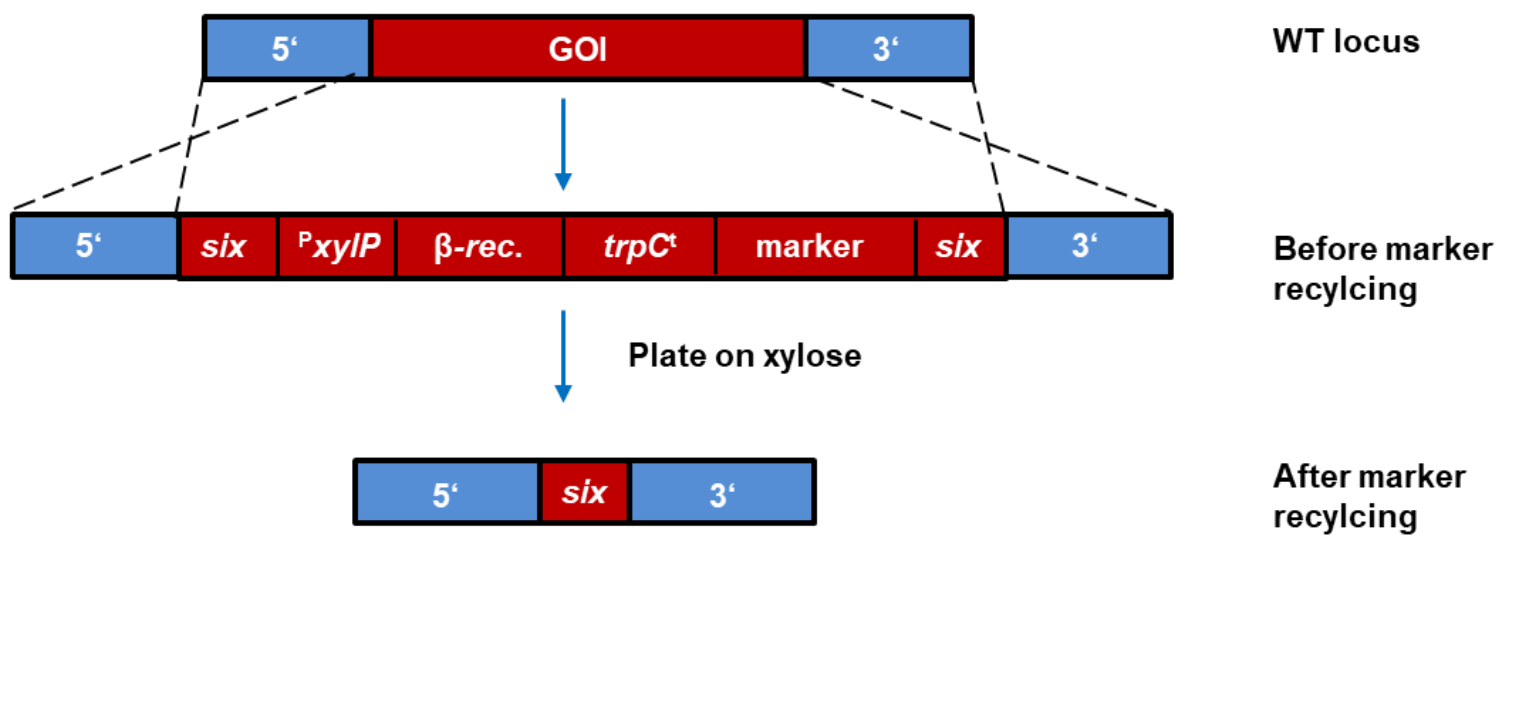

FIGURE 12: Schematic illustration of integration and recycling of a recyclable marker cassette.

The scheme depicts a gene deletion as example. The open reading frame (ORF) of the gene of interest (GOI) is replaced by homologous recombination by a recyclable marker cassette onlocus. The recyclable marker cassette contains the $\beta$-serine recombinase gene ( $\beta$-rec.), which is driven by the inducible xylose promoter $\left({ }^{\mathrm{P}} x y / P\right)$ and employs the $\operatorname{trp} C$ terminator $\left(\operatorname{trp} C^{t}\right)$. The addition of xylose to the minimal medium induces the expression of ${ }^{\mathrm{P}} x y / P:: \beta-r e c$, which leads to the excision of the whole cassette from the fungal genome. Only a small six recognition site remains as a scar. 5' and 3' indicate the untranslated regions flanking the ORF (adapted from (Hartmann et al., 2010)).

\subsubsection{Control of the plasmids by sequencing}

Plasmids created in this study were sequenced by SEQLAB SEQUENCE LABORATORIES GMBH (Göttingen, Germany). Analysis of obtained sequences was done with the Lasergene software package (DNA STAR INC.). All plasmids, which were constructed in this study and cloned in pBluescript SK+ were sequenced with the primers kt182, kt183, kt184 and kt339. These primers bind near both six sites of the recyclable marker cassettes and close to the EcoRV cloning site of pBluescript SK+. Further primers are indicated in the respective sub-CHAPTERS 2.5.5.

\subsubsection{Plasmid and strain construction of $\boldsymbol{A}$. nidulans mutant strains}

The DNA fragment sizes given in the forthcoming sections are rounded. Genomic DNA of FGSC A4 ( $A$. nidulans WT, ve $\left.A^{+}\right)$was used as template, if not stated otherwise. 


\subsubsection{Construction of the $\operatorname{vos} A \Delta$ cassette and the vosA $\Delta$ strain}

$1.6 \mathrm{~kb}$ of the 5 ' flanking region of $\operatorname{vos} A$ was amplified with primers SR11/12, which introduce $15 \mathrm{bp}$ overhangs to the EcoRV restriction site of pBluescript SK+ and the six site, respectively. The respective $1.5 \mathrm{~kb}$ long 3' region was amplified with primers SR13/14, which introduce $15 \mathrm{bp}$ overhangs homologous to the six site and pBluescript $\mathrm{SK}+$. Both sequences and the natRM recyclable marker cassette were cloned into the $E c o R V$ multiple cloning of pBluescript $\mathrm{SK}+$ in a seamless cloning reaction, resulting in pME4603. Subsequently, the deletion cassette was excised with Mssl and transformed into AGB551, resulting in the strain AGB1057. The correct substitution of the original gene with the deletion construct was verified by Southern hybridization.

\subsubsection{Plasmid and strain construction of velB $\Delta$}

For construction of pME4604 for the velB $A$. nidulans strain $2 \mathrm{~kb}$ of the 5 ' region was amplified with primers SR05/06, including 15 bp overhangs to the EcoRV restriction site of pBluescript SK+ and the six site of the recyclable marker, respectively. $1.6 \mathrm{~kb}$ of the 3' flanking region was amplified with primers SR07/08, which introduce 15 bp overhangs homologous to the six site and pBluescript SK+. Both sequences and the natRM recyclable marker cassette were cloned into the EcoRV multiple cloning of pBluescript $\mathrm{SK}+$ in a seamless cloning reaction. After excision with Mssl, the velB deletion construct was transformed into AGB551, resulting in AGB1064. The correct on-locus integration into $A$. nidulans genome was confirmed by Southern hybridization.

\subsubsection{Construction of the vel $B^{I D D_{\Delta}}$ cassette and the vel $B^{I D D_{\Delta}}$ strains}

For construction of a $v e l B^{I D D_{\Delta}}$ strain $1.8 \mathrm{~kb}$ including $1.3 \mathrm{~kb}$ of the 5 ' region and half of the velvet domain of the velB gene (until the IDD) was amplified from A. nidulans FGSC A4 gDNA. Therefore, primers SR110/109 were employed, introducing overhangs of 15 bp homologous to pBluescript SK+ and to the second half of velB velvet domain. This second half of the velB velvet domain with a size of $0.5 \mathrm{~kb}$ was amplified with SR108/111 with a homologous $15 \mathrm{bp}$ overhang to the six site of the recyclable marker cassette. The respective $1.6 \mathrm{~kb}$ long 3' region was amplified with primers SR112/113 likewise introducing overhangs of $15 \mathrm{bp}$ to the six site of the recyclable marker cassette and pBluescript SK+. This three sequences and the phleoRM cassette were cloned into the EcoRV multiple cloning site of pBluescript $\mathrm{SK}+$ in a seamless cloning reaction according to manufacturer's conditions, resulting in pME4686. The plasmid was sequenced with additional primers SR26 and SR108. The construct containing the truncated velB gene was excised with Mssl and transformed into AGB551, resulting in the 
strain AGB1131. Successful transformation at the correct locus was verified by Southern hybridization.

\subsubsection{Plasmid and strain constructions of VelB-GFP fusion construct}

sgfp was amplified with primers SR18/20 from pME4292, introducing a 15 bp linker region between the velB gene and the sgfp gene. A $3.3 \mathrm{~kb}$ fragment harboring $2 \mathrm{~kb}$ of the 5 ' flanking region and the velB gene was amplified with primers SR05/24, introducing overhangs of $15 \mathrm{bp}$ to the pBluescript SK+ backbone and to the sgfp fragment. $1.6 \mathrm{~kb}$ of the 3' flanking region was amplified with primers SR07/08, which introduce 15 bp overhangs homologous to the six site and pBluescript SK+. These three fragments and the natRM marker cassette were cloned into the EcoRV multiple cloning site of pBluescript $\mathrm{SK}+$ in a seamless cloning reaction, resulting in plasmid pME4687. The primers SR25, SR26, JG826 and JG843 were used for sequencing. The plasmid was linearized by cutting the desired construct with Mssl, followed by transformation in AGB551, which resulted in the $A$. nidulans strain AGB1132. Furthermore, the fusion construct pME4687 was transformed into AGB817, resulting in AGB1139. Southern hybridization was conducted to proof the correct integration at the correct locus.

\subsubsection{Construction of the velBIDD ${ }^{\Delta}: g f p$ fusion construct and the velB ${ }^{I D D \Delta}:: g f p$ mutant strain}

Fragments used for pME4686 were used for the construction of this construct, but the second half of the velB velvet domain was amplified with primers SR108/24 to introduce a $15 \mathrm{bp}$ overhang to the sgfp gene. The velB velvet domain fragments amplified with SR109/110 and SR108 were joined by fusion PCR. This desired fragment was together with the sgfp gene (amplified with SR18/20 from pME4292), the 3' flanking region and the phleoRM marker cassette cloned into the EcoRV multiple cloning site of pBluescript SK+. This results in the plasmid pME4688. The plasmid was sequenced with additional primers SR26 and SR108. Additional primers JG826 and SR26 were used for sequencing of the plasmid. Subsequently, the plasmid was cut with MssI and transformed in AGB551, yielding AGB1133. Employment of Southern hybridization allowed the verification of successful transformation.

\subsubsection{Plasmid and strain construction of VelB ${ }^{\text {A.f._.IDD }}$ complementation}

The $1.6 \mathrm{~kb}$ 3' flanking region was amplified with SR112/113 and cloned into the Eco72I restriction site of pME4305, resulting in pME4689. For construction of a velB ${ }^{A . f .} \_I D D$ complementation strain $1.8 \mathrm{~kb}$ including $1.3 \mathrm{~kb}$ of the $5^{\prime}$ region and half of the velvet domain of the velB gene (until the IDD) was amplified with primers SR110/253 from $A$. nidulans FGSC A4 
gDNA. The $0.3 \mathrm{~kb}$ velB ${ }^{I D D}$ of $A$. fumigatus was amplified with primers SR266/267 from $A$. fumigatus Afs35 gDNA. This second half of the velB velvet domain with a size of $0.5 \mathrm{~kb}$ was amplified with SR108/111 with a homologous 15 bp overhang to the six site of the recyclable marker cassette. All fragments contain 15 bp overhangs respectively to each other to join them by fusion PCR. The fused fragment was cloned into the Swal restriction site of pME4689, resulting in pME4690. Additionally the plasmid was sequenced with primer SR26. After excision with Mssl the linearized construct was transformed into AGB1133, resulting in AGB1134. The correct integration of the construct was confirmed by Southern hybridization.

\subsubsection{Plasmid and strain construction of VelB ${ }^{\text {V.d._.IDD }}$ complementation}

The $1.6 \mathrm{~kb}$ 3' flanking region was amplified with SR112/113 and cloned into the Eco72I restriction site of pME4305, resulting in pME4689. For construction of a velB ${ }^{V . d . . I D D}$ complementation strain $1.8 \mathrm{~kb}$ including $1.3 \mathrm{~kb}$ of the $5^{\prime}$ region and half of the velvet domain of the velB gene (until the IDD) was amplified with primers SR110/253 from A. nidulans FGSC A4 gDNA. The $0.4 \mathrm{~kb}$ vel2 $2^{I D D}$ of $V$. dahliae was amplified with primers SR254/255 from $V$. dahliae JR2 gDNA. This second half of the velB velvet domain with a size of $0.5 \mathrm{~kb}$ was amplified with SR108/111 with a homologous $15 \mathrm{bp}$ overhang to the six site of the recyclable marker cassette. All fragments contain 15 bp overhangs respectively to each other to join them by fusion PCR. The fused fragment was cloned into the Swal restriction site of pME4689, resulting in pME4691. The plasmid was additionally sequenced with primer SR26. The construct was cut with Mssl and transformed into AGB1133, yielding AGB1135. Southern hybridization was conducted for verification of the integration of the desired construct on-locus of $A$. nidulans genome.

\subsubsection{Construction of $\operatorname{vos} A:$ ha plasmid and respective co-IP strains}

$3.4 \mathrm{~kb}$ including $1.4 \mathrm{~kb}$ of the 5 ' flanking region and the vos $A$ gene was amplified with primers SR76/201, introducing on the one side a 15 bp overhang to the pBluescript SK+ and on the other side an overhang of $45 \mathrm{bp}$ including nucleotides encoding the HA (hemaglutinin antigen) tag (30 bp) and the $15 \mathrm{bp}$ overhang to the six site of the marker cassette, respectively. The 1.4 kb 3' flanking region was amplified with primers SR49/75. The two fragments and the phleoRM marker cassette were cloned into the $E c o R V$ multiple cloning site of pBluescript $\mathrm{SK}_{+}$, resulting in pME4693. The primers SR86, SR87 and SR96 were additionally employed for sequencing. The plasmid was excised with Mssl and transformed into AGB1132 and AGB1133, resulting in AGB1136 and AGB1137, respectively. Successful transformation and correct integration into the genome was verified by Southern hybridization. 


\subsubsection{Construction of $v e l B^{I D D} \Delta /$ velvet $\Delta$ double mutant strains}

pME4688 was cut with Mssl and transformed into AGB1006, AGB1062 and AGB1057, resulting in AGB1140, AGB1141 and AGB1142, respectively. The correct on-locus integration into $A$. nidulans genome was confirmed by Southern hybridization.

\subsubsection{Plasmid and strain construction of VelC-GFP fusion construct}

$4.1 \mathrm{~kb}$ including $2.3 \mathrm{~kb}$ of the 5 ' flanking region and the velC gene were amplified with primers kt203/SR56, which introduces 15 bp overhangs to the pBluescript SK+ and to the sgfp gene amplified with SR18/20 from pME4292. These two fragments were joined by fusion PCR. 2.1 $\mathrm{kb}$ of the 3' region was amplified with SR57/kt204, introducing 15 bp overhangs to six site of the marker cassette and the pBluescript SK+. These fragments and the natRM recyclable marker cassette were cloned into the EcoRV multiple cloning site of pBluescript $\mathrm{SK}_{+}$, which results in the plasmid pME4693. Additional primers SR88, SR89, SR97, SR98, SR99, JG826 and JG466 were used for sequencing. After excision with Mssl, the construct was transformed into AGB1062 to complement the velC deletion strain, resulting in AGB1137. Successful transformation and complementation was verified by conducting Southern hybridization.

\subsubsection{Construction of velvet deletion strains}

Transformation of pME4604 into AGB1062 and AGB1066 resulted in the double mutant strains AGB1143 and AGB1144, respectively. Subsequent transformation of pME4603 yielded the triple deletion mutant strain AGB1145 and transformation of pME4602 in this strain resulted in the quadruple velvet deletion mutant strain AGB1146.

\subsubsection{Plasmid construction of $v e l B^{I D D} \Delta$ ::his for recombinant expression in $E$. coli}

The $0.5 \mathrm{~kb}$ fragments for the two parts of the velB velvet domain were amplified from pME3815 with primers JG45/SR109 and JG46/SR108. These fragments were joined by fusion PCR containing an overhang for the $\mathrm{Ncol}$ or Xhol restriction site, respectively. After subcloning in the pJET plasmid, the construct was excised with $\mathrm{Ncol}$ and $\mathrm{Xhol}$ and cloned into $\mathrm{Ncol} / \mathrm{Xhol}$ site of pETM-13, which contains a sequence encoding a C-terminal His-tag, resulting in pME4692. 


\subsection{Genetic manipulations of microorganisms}

\subsubsection{Transformation of $E$. coli}

E. coli transformations were executed as described in (Hanahan et al., 1991; Inoue et al., 1990). In brief, plasmid DNA was added to chemically competent $E$. coli cells and incubated for $30 \mathrm{~min}$ on ice. Subsequently, cells were heat-shocked at $42^{\circ} \mathrm{C}$ to allow plasmid uptake followed by a cooling step on ice for one to two minutes. $800 \mu \mathrm{L}$ LB was added and cultures were shaken for 30 to $60 \mathrm{~min}$ at $37^{\circ} \mathrm{C}$ on a rotary shaker. Finally, E. coli cells were collected by centrifugation, inoculated on LB plates supplemented with 1:1000 ampicillin and incubated o/n at $37^{\circ} \mathrm{C}$. The addition of ampicillin prevents plasmid loss and enables the selection of positive clones, which contain the desired plasmid.

\subsubsection{Transformation of $\boldsymbol{A}$. nidulans}

For transformation in A. nidulans polyethylene glycol-mediated protoplast fusion was employed as described before (Punt and van den Hondel, 1992). In the presented study A. nidulans AGB551 (Bayram et al., 2012) or AGB551 derived strains were used as transformation hosts for all genetic modifications and AGB551 was used as wildtype. The deletion of the nkuA gene in the transformation hosts remarkably increases homologous recombination during transformation, resulting in on-locus integration of linearized constructs (Krappmann et al., 2006b; Nayak et al., 2005).

Host strains were grown o/n in submerged cultures at $37^{\circ} \mathrm{C}$ on a rotary shaker. Mycelia were harvested through sterile Miracloth filters, washed with sterile citrate buffer $(150 \mathrm{mM} \mathrm{KCl}$, $580 \mathrm{mM} \mathrm{NaCl}, 50 \mathrm{mM} \mathrm{Na}$-citrate $\mathrm{pH}$ 5.5) and transferred into sterile Erlenmeyer flasks containing sterile filtered protoplastation solution $\left(30 \mathrm{mg} / \mathrm{mL}\right.$ Vinoflow ${ }^{\circledR}$ Max or Vinotaste ${ }^{\circledR}$ Pro from NovOZYMES (Bagsvaerd, Denmark) and $15 \mathrm{mg} / \mathrm{mL}$ lysozyme (SERVA) dissolved in citrate buffer and sterile filtered through $0.2 \mu \mathrm{m}$ filters (SARSTEDT). Mycelia were incubated for 100 min at $30^{\circ} \mathrm{C}$ under constant slow agitation for protoplastation and protoplast formation was monitored by microscopy. Subsequently, protoplasts were harvested through sterile Mircacloth filters into $50 \mathrm{~mL}$ pre-cooled centrifuge tubes (SARSTEDT) and the volume filled up to $50 \mathrm{~mL}$ with ice-cold STC 1700 buffer (1.2 M sorbitol, $10 \mathrm{mM}$ Tris pH 5.5, $50 \mathrm{mM} \mathrm{CaCl}_{2}, 35 \mathrm{mM} \mathrm{NaCl}$ ). Protoplasts were centrifuged at $2600 \mathrm{rpm}$ for $12 \mathrm{~min}$ at $4^{\circ} \mathrm{C}$ and washed twice with ice cold STC1700. Washed protoplasts were incubated with approximately $10 \mathrm{ng}$ of respective linearized DNA constructs for $30 \mathrm{~min}$ in ice. Afterwards, $1.35 \mathrm{~mL}$ sterile PEG solution (10 mM Tris $\mathrm{pH} 7.5,50 \mathrm{mM} \mathrm{CaCl}_{2}, 60 \%$ (v/v) PEG4000) was added stepwise to the protoplast-DNA mixture, to enhance DNA uptake, and incubated for $40 \mathrm{~min}$ over the ice. Finally, protoplasts were centrifuged at $2600 \mathrm{rpm}$ for $15 \mathrm{~min}$ at $4^{\circ} \mathrm{C}$, carefully spread on solid MM supplemented with respective selecting agents (nourseothricin 0.7:1000, phleomycin 1:1000) and incubated 
at $37^{\circ} \mathrm{C}$. After three to seven days transformed clones were picked and individualized on selective MM plates. Southern hybridization was employed for the verification of successful transformation of constructs. The recyclable marker cassettes were removed from the genome of respective mutants by singularizing clones on $\mathrm{MM} / \mathrm{xylose}$ plates $(0.5 \%(\mathrm{w} / \mathrm{v})$ glucose, $0.5 \%$ (w/v) xylose, $7 \mathrm{mM} \mathrm{KCl}, 2 \mathrm{mM} \mathrm{MgSO} 4,70 \mathrm{mM} \mathrm{NaNO}_{3}, 11.2 \mathrm{mM} \mathrm{KH}_{2} \mathrm{PO}_{4}, 0.1 \%$ (v/v) trace element solution pH 5.5) (Hartmann et al., 2010). Successful recycling of the marker cassette was confirmed with Southern hybridization.

\subsection{Southern hybridization}

Successful integration of transformed constructs was validated by the employment of Southern hybridization. Restriction enzymes (Thermo Fisher Scientific) were used as instructed in manufacturer's protocol. Genomic DNA of respective $A$. nidulans mutant strains was digested with restriction enzymes o/n, which were selected the way that deriving DNA fragments span parts of the respective mutated locus. Furthermore, at least one restriction site was outside of the integrated construct to confirm on-locus integration. An important selection criterion was also that respective DNA fragments show clear size differences between mutant and host strains when separated by agarose gel electrophoresis. Separation of DNA fragments according to size by agarose gel electrophoresis was followed by washing of gels for $10 \mathrm{~min}$ in wash buffer $1(0.25 \mathrm{M} \mathrm{HCl})$, followed by washing with buffer 2 for denaturation $(0.5 \mathrm{M} \mathrm{NaOH}$, $1.5 \mathrm{M} \mathrm{NaCl})$ for $25 \mathrm{~min}$ and $30 \mathrm{~min}$ in buffer $3(0.5 \mathrm{M}$ Tris, $1.5 \mathrm{M} \mathrm{NaCl}, \mathrm{pH} 7.4)$ for neutralizing. The performance of all three washing steps was done under constant agitation at rt. After washing, DNA was transferred onto Amersham ${ }^{\mathrm{TM}}$ Hybond $^{\mathrm{TM}}-\mathrm{N}$ nylon membranes (GE HEALTHCARE) by dry-blotting for $2 \mathrm{~h}$ at rt. Subsequently, membranes were dried for $10 \mathrm{~min}$ at $75^{\circ} \mathrm{C}$ and DNA was cross-linked to the membrane by UV light exposure $(\lambda=254 \mathrm{~nm})$ for 3 min per side. Pre-hybridization of membranes was conducted with hybridization solution from the Amersham $^{\mathrm{TM}}$ Gene Images AlkPhos Direct Labelling and Detection System (GE HEALTHCARE, prepared after manufacturer's instructions) for $30 \mathrm{~min}$ at $55^{\circ} \mathrm{C}$ in a HERA hybrid $\mathrm{R}$ hybridization oven (HERAEUS INSTRUMENTS) before addition of the DNA probe. Preparation of DNA probes was performed with the aforementioned kit according to manufacturer's instructions. Membranes were hybridized with respective DNA probes $\mathrm{o} / \mathrm{n}$ at $55^{\circ} \mathrm{C}$. The next day, membranes were washed two times in post-hybridization buffer I $\left(1 \mathrm{mM} \mathrm{MgCl}_{2}, 3.5 \mathrm{mM}\right.$ SDS, $50 \mathrm{mM}$ sodium phosphate buffer, $150 \mathrm{mM} \mathrm{NaCl}, 2 \mathrm{M}$ Urea, $0.2 \%$ (w/v) blocking reagents) for $10 \mathrm{~min}$ at $55^{\circ} \mathrm{C}$ and two times in post-hybridization buffer II $(2 \mathrm{mM} \mathrm{MgCl}, 50 \mathrm{mM}$ Tris, $100 \mathrm{mM} \mathrm{NaCl}, \mathrm{pH} \mathrm{10)}$ for $5 \mathrm{~min}$ at rt under constant agitation. Application of CDP-Star (GE HEALTHCARE) enables detection of DNA bands, which become visible after exposing the membranes to Amersham ${ }^{\mathrm{TM}}$ Hyperfilm $^{\mathrm{TM}}$ ECL (GE HEALTHCARE). 


\subsection{Secondary metabolite extraction}

\subsubsection{Sterigmatocystin isolation}

Sterigmatocystin isolation and thin layer chromatography (see next CHAPTER) were performed as described (Bayram et al., 2008). 1*105 spores were distributed on solid MM and grown for three or seven days at $37^{\circ} \mathrm{C}$ in light or dark. Two agar plugs were excised with a $50 \mathrm{~mL}$ centrifuge tube (SARSTEDT), which was cut into small pieces. The agar pieces were transferred into $50 \mathrm{~mL}$ Falcon tubes and six glass beads and $3 \mathrm{~mL} \mathrm{H}_{2} \mathrm{O}$ were added. Samples were shaken roughly for $30 \mathrm{~min}$ at rt. Subsequently, $3 \mathrm{~mL}$ chloroform was added and samples were shaken for another $30 \mathrm{~min}$ at rt. This was followed by centrifugation at $1000 \mathrm{rpm}$ for $10 \mathrm{~min}$ at $4^{\circ} \mathrm{C}$ for phase separation and the lower chloroform metabolite-containing chloroform phase was transferred into glass tubes and evaporated o/n at $r$ under the hood.

\subsubsection{Thin layer chromatography}

Samples of sterigmatocystin isolation were resuspended in $50 \mu \mathrm{L}$ methanol and three times $5 \mu \mathrm{L}$ of isolated sterigmatocystin per sample was applied spot-wise to pre-coated SIL G/UV254 Polygram ${ }^{\circledR}$ DC-foil TLC-sheets (MACHEREY-NAGEL) (thin layer chromatography plates). TLC plates were run in 1:4 (v/v) acetone:chloroform for 40-50 min, dried for $5 \mathrm{~min}$ and photographed at 366 and $254 \mathrm{~nm}$ with a Camag TLC Visualizer 2 system from CAMAG (Muttenz, Switzerland). Afterwards, TLC plates were sprayed with $20 \%$ (v/v) aluminum chloride in $95 \%(\mathrm{v} / \mathrm{v})$ ethanol and incubated at $70^{\circ} \mathrm{C}$ for $10 \mathrm{~min}$. Derivatized TLC plates were photographed again at 366 and $254 \mathrm{~nm}$ UV light and white light with a Camag TLC visualizer 2 system and processed with the winCATS 1.4 .4 software (CAMAG).

\subsubsection{Secondary metabolite extraction for HPLC and LC-MS/MS measurements}

For secondary metabolite extraction $1^{*} 10^{5}$ spores were distribute over the whole agar plate and grown for three days at $37^{\circ} \mathrm{C}$ in light or dark, promoting asexual or sexual development, respectively. Subsequently, fungal cells were removed thoroughly with cotton swabs into $0.96 \% \mathrm{NaCl}$ solution, containing $0.0002 \%(\mathrm{v} / \mathrm{v})$ Tween. The cell-free agar was excised into small pieces, transferred to $500 \mathrm{~mL}$ flasks. The agar pieces were shaken in $300 \mathrm{~mL}$ ethyl acetate at $160 \mathrm{rpm}$ at $30^{\circ} \mathrm{C}$ for $30 \mathrm{~min}$. This was followed by $15 \mathrm{~min}$ ultra-sonication in a Bandelin Sonorex ${ }^{\mathrm{TM}}$ Digital 10P ultrasonic bath from BANDELIN ELECTRONIC GMBH AND CO. KG (Berlin, Germany) at highest level. Ethyl acetate was transferred (without agar pieces) to round bottom flasks and evaporated in a Hei-VAP-Advantage rotary evaporator from HEIDOLPH INSTRUMENTS GMBH \& CO. KG (Schwabach, Germany) with a MWG Lauda RM6 from LAUDA- 
BRINKMANN LP (Delran, NJ, USA) and a Laboxact KNF vacuum system (SIGMA-ALDRICH) at $37^{\circ} \mathrm{C}$ under constant gyration.

Subsequently, extracted secondary metabolites were resolved in $3 \mathrm{~mL}$ methanol, transferred into small glass tubes and evaporated in a rotary evaporator at $37^{\circ} \mathrm{C}$ under constant gyration. Samples were stored at $-20^{\circ} \mathrm{C}$ and before high performance liquid chromatography (HPLC) measurements resolved $500 \mu \mathrm{L}$ methanol, centrifuged and $250 \mu \mathrm{L}$ taken for measurements.

\subsubsection{Secondary metabolite analysis by high-performance liquid chromatography (HPLC) coupled with a UV diode array detector (UV-DAD)}

Analytical HPLC/UV-DAD measurements were performed using the following system: HPLC pump 420, SA 360 autosampler, Celeno UV-DAD HPLC detector, ELSD-Sedex 85 evaporative light-scattering detector (ERC)) with a Nucleodur 100-5 C18 end-capped (ec) column (250 mm x $3 \mathrm{~mm}$ ) and the solvent system: $A=\mathrm{H} 2 \mathrm{O}+0.1 \%(\mathrm{v} / \mathrm{v})$ trifluoroacetic acid (TFA), $B=$ acetonitrile $+0.1 \%(\mathrm{v} / \mathrm{v})$ TFA (from GoebEL INSTRUMENTELLE ANALYTIK GMBH, Au/Hallertau, Germany). Secondary metabolite extracts were dissolved in $500 \mu \mathrm{L}$ methanol and an injection volume of $20 \mu \mathrm{L}$ was analyzed under gradient conditions (20\% B to $100 \%$ B in 20 minutes) with a flow rate of $0.5 \mathrm{~mL} / \mathrm{min}$.

HPLC data was analyzed with the Geminyx III software from GoEBEL INSTRUMENTELLE ANALYTIK GmbH (Au/Hallertau, Germany).

\subsubsection{Secondary metabolite analysis by liquid chromatography coupled to tandem mass spectrometry (LC-MS/MS)}

Crude extracts from secondary metabolite isolation were solved in $500 \mu \mathrm{L}$ methanol and analyzed using a Dionex Ultimate 3000 system (THERMO SCIENTIFIC) connected to an Impact II qTof mass spectrometer (Bruker) for UHPLC-UV and UHPLC-ESI-HRMS/MS analysis. $5 \mu \mathrm{L}$ of each sample was injected for separation on an UHPLC reversed phase column (Acquity UPLC BEH C18 1.7 ImRP 2.1 x50 mm column (WATERS) with an Acquity UPLC BEH C18 1.7 ImRP $2.1 \times 5 \mathrm{~mm}$ pre-column (WATERS)) applying a linear acetonitrile/0.1\% (v/v) formic acid in $\mathrm{H}_{2} \mathrm{O} / 0.1 \%(\mathrm{v} / \mathrm{v})$ formic acid gradient (from $5 \%$ to $95 \%(\mathrm{v} / \mathrm{v})$ acetonitrile/0.1 formic acid in 19 min) with a flow rate of $0.6 \mathrm{~mL} / \mathrm{min}$ at $30^{\circ} \mathrm{C}$. The measurements were performed in a mass range of $100-1200 \mathrm{~m} / \mathrm{z}$ in positive or negative mode. For internal mass calibration a $10 \mathrm{mM}$ sodium formate solution was employed. Data analysis and sum formula predictions were performed with Bruker Compass DataAnalysis 4.3. 


\subsection{Protein methods}

\subsubsection{Protein isolation}

$1^{*} 10^{7}$ spores were inoculated in $\mathrm{MM}$ and strains were grown vegetatively in submerged cultures. Protein isolation of asexually or sexually grown cultures, strains were grown vegetatively for $24 \mathrm{~h}$ and subsequently shifted onto solid MM plates and grown for indicated time points in light or dark. For cycloheximide assay, after $24 \mathrm{~h}$ of vegetative growth, $250 \mu \mathrm{L}$ cycloheximide $(10 \mathrm{mg} / \mathrm{mL})$ was added to the cultures, which were incubated for 0 to $5 \mathrm{~h}$ prior protein extraction. Mycelia were collected with sterile Miracloth filter, washed with sterile $0.96 \%$ (w/v) $\mathrm{NaCl}$ supplemented with $1 \mathrm{mM}$ PSMF and 1\% (v/v) Dimethyls frozen in liquid nitrogen. Frozen mycelia were ground in liquid nitrogen with a MM400 table mill (RETSCH) and approximately $200 \mathrm{mg}$ was mixed with $300 \mu \mathrm{L} \mathrm{B}{ }^{+}$buffer $(300 \mathrm{mM} \mathrm{NaCl}, 100 \mathrm{mM}$ Tris $\mathrm{pH} 7.5,10 \%(\mathrm{v} / \mathrm{v})$ glycerol, $1 \mathrm{mM}$ EDTA, 0.1\% (v/v) NP-40) supplemented with $1.5 \mathrm{mM}$ DTT, 1 tablet/50 mL complete EDTA-free protease inhibitor cocktail (ROCHE), $1 \mathrm{mM}$ PMSF, phosphatase inhibitor $\operatorname{mix}(1 \mathrm{mM} N \mathrm{NaF}, 0.5 \mathrm{mM}$ sodium-orthovanadate, $8 \mathrm{mM}$ $\beta$-glycerolphosphate disodium pentahydrate and $1.5 \mathrm{mM}$ benzamidine) and centrifuged at 13000 for $15 \mathrm{~min} r p m$ at $4^{\circ} \mathrm{C}$. Subsequently, supernatant was transferred into fresh $1.5 \mathrm{~mL}$ reaction tubes and protein concentration was measured with a NanoDrop ND-1000 spectrophotometer (PEQLAB). Protein concentration of samples were adjusted to same values with $\mathrm{B}^{+}$buffer and samples were mixed with $3 x$ SDS sample buffer $(250 \mathrm{mM}$ Tris- $\mathrm{HCl} \mathrm{pH} 6.8$, $15 \%$ (v/v) $\beta$-mercaptoethanol, 30\% (v/v) glycerol, 7\% (v/v) SDS, 0.3\% (w/v) bromphenol blue) and boiled at $95^{\circ} \mathrm{C}$ for 5 min followed by 5 min incubation on ice. Samples were stored at $20^{\circ} \mathrm{C}$ or directly used for further experiments.

\subsubsection{SDS-PAGE and western hybridization}

SDS-polyacrylamide gel electrophoresis (SDS-PAGE) was employed for separation of proteins according to size and for transferring proteins to nitrocellulose membranes for western hybridization (Laemmli, 1970; Schinke et al., 2016). Protein concentrations were determined with a NanoDrop ND-1000 photospectrometer (THERMO FISHER SCIENTIFIC) and equal amounts of proteins were loaded on 12\% SDS gels (separation gel: $2.8 \mathrm{~mL} \mathrm{H}_{2} \mathrm{O}, 3.75 \mathrm{~mL} 1 \mathrm{M}$ Tris $\mathrm{pH}$ 8.8, $100 \mu \mathrm{L}$ 10\% (w/v) SDS, $3.3 \mathrm{ml}$ 30\% (v/v) acrylamide, $10 \mu \mathrm{L}$ TEMED, $50 \mu \mathrm{L}$ 10\% (w/v) APS; stacking gel: $3.67 \mathrm{~mL} \mathrm{H} \mathrm{H}_{2} \mathrm{O}, 625 \mu \mathrm{L} 1 \mathrm{M}$ Tris pH 6.8, $30 \mu \mathrm{L}$ 10\% (w/v) SDS, $650 \mu \mathrm{L} 30 \%$ $(\mathrm{v} / \mathrm{v})$ acrylamide, $5 \mu \mathrm{L}$ TEMED, $25 \mu \mathrm{L} 10 \%(\mathrm{w} / \mathrm{v})$ APS) and separated according to size at 200 $\mathrm{V}$ in running buffer (25 mM Tris, $0.25 \mathrm{M}$ glycine, $0.1 \%$ (w/v) SDS). For western hybridization proteins were blotted for $1 \mathrm{~h}$ at $100 \mathrm{~V}$ on Amersham ${ }^{\mathrm{TM}}$ Protran $^{\mathrm{TM}} 0.45 \mu \mathrm{m} \mathrm{NC}$ nitrocellulose membranes (GE HEALTHCARE) in ice cooled transfer buffer (25 mM Tris, $192 \mathrm{mM}$ glycine, $0.02 \%(\mathrm{w} / \mathrm{v}) \mathrm{SDS}$ ) or at $35 \mathrm{~V}$ o/n at it in transfer buffer (Towbin et al., 1979). Membranes were blocked with $5 \%$ (w/v) skim milk powder dissolved in TBST buffer (10 mM Tris- $\mathrm{HCl} \mathrm{pH}$ 8.0, 150 
$\mathrm{mM} \mathrm{NaCl}, 0.05 \%(\mathrm{v} / \mathrm{v})$ Tween 20$)$ for $1 \mathrm{~h}$ at $\mathrm{rt}$ and subsequently probed with one of the first antibodies, listed in CHAPTER 2.1, in TBST-M (TBST buffer, supplemented with $5 \%(\mathrm{w} / \mathrm{v})$ skim milk powder) and incubated $\mathrm{o} / \mathrm{n}$ at $4^{\circ} \mathrm{C}$. One the following day, membranes were washed three times in TBST for $10 \mathrm{~min}$ at $\mathrm{rt}$ under constant agitation and incubated for $1 \mathrm{~h}$ with 1:1000 horseradish peroxidase coupled goat a-mouse (or a-rabbit, respectively) antibody as secondary antibody in TBST-M. Membranes were washed three times for 10 min with TBST at rt under constant agitation. Finally, membranes were covered with a $1: 1(\mathrm{v} / \mathrm{v})$ mixture of solution $\mathrm{A}(2.5 \mu \mathrm{M}$ luminol, $400 \mu \mathrm{M}$ paracoumarat, $100 \mathrm{mM}$ Tris- $\mathrm{HCl} \mathrm{pH} 8.5)$ and solution $\mathrm{B}$ (5.4 mM H2O2, $100 \mathrm{mM}$ Tris- $\mathrm{HCl} \mathrm{pH} 8.5$ ) and incubated for 5 min under constant agitation at $\mathrm{rt}$ in the dark (Suck and Krupinska, 1996). Detection of chemiluminescent signals was done by employing a Fusion-SL7 chemiluminescence detection system (PEQLAB) and pictures were recorded with the Fusion 15.15 software from VILBER LOURMAT (Marne-la-Vallée cedex 3, France). As loading control membranes were stained with Ponceau staining (Romero-Calvo et al., 2010). For utilization of $\alpha-V^{-} I^{I D D}$ antibody all steps were performed with TBS (10 mM Tris- $\mathrm{HCl} \mathrm{pH} \mathrm{8.0,150} \mathrm{mM} \mathrm{NaCl),} \mathrm{instead} \mathrm{of} \mathrm{TBST.}$

\subsubsection{Protein pull downs}

\subsubsection{Immunoprecipitation with GFP-tagged fusions proteins}

Protein GFP pull downs were conducted by employing GFP-Trap ${ }^{\circledR}$ A beads from CHROMOTEK (Planegg-Martinsried, Germany) as described earlier (Jöhnk et al., 2016). $5^{\star} 10^{8}$ spores of A. nidulans strains were inoculated in $500 \mathrm{~mL}$ MM and grown for $24 \mathrm{~h}$ in submerged cultures (vegetative samples) at $37^{\circ} \mathrm{C}$. Sexual samples were shifted afterwards to solid MM, sealed with Parafilm ${ }^{\circledR}$ and incubated for $48 \mathrm{~h}$ in the dark at $37^{\circ} \mathrm{C}$. For GFP trap mycelia were harvested, washed with $0.96 \%(\mathrm{w} / \mathrm{v}) \mathrm{NaCl}$ supplemented with $1 \mathrm{mM}$ PMSF and $1 \%(\mathrm{v} / \mathrm{v})$ DMSO, dried and immediately frozen in liquid nitrogen. Mycelia were ground with a MM400 Table mill (RETSCH) and $5 \mathrm{~mL}$ ground mycelia were collected in a pre-frozen $15 \mathrm{~mL}$ centrifugation tube. Subsequently, $5 \mathrm{~mL} \mathrm{~B}^{+}$buffer was added, thoroughly mixed with the mycelia, which was followed by centrifugation of $20 \mathrm{~min}$ at $4000 \mathrm{rpm}$ and $4^{\circ} \mathrm{C}$ twice. The supernatant was filtered through $0.2 \mu \mathrm{m}$ sterile filters (SARSTEDT), GFP-Trap ${ }^{\circledR}$ A beads, which were previously equilibrated in $\mathrm{B}^{+}$buffer, were added (CHROMOTEK) in a relation of $1: 1(\mathrm{v} / \mathrm{v})$ and incubated rotating for $3 \mathrm{~h}$ or $\mathrm{o} / \mathrm{h}$ at $4^{\circ} \mathrm{C}$. After incubation beads were treated in two different ways. 1) Beads were washed twice with fresh $\mathrm{B}^{+}$buffer, transferred into $1.5 \mathrm{~mL}$ reaction tubes and centrifuged at for $1 \mathrm{~min}$ at $3000 \mathrm{rpm}$ at rt. The supernatant was removed and the beads were boiled in $50 \mu \mathrm{L} 3 \mathrm{x}$ SDS samples buffer for $10 \mathrm{~min}$ at $95^{\circ} \mathrm{C}$. 2) The supernatant with the GFP beads was loaded onto fresh Poly-Prep ${ }^{\circledR}$ Chromatography Columns (BIO-RAD), which were equilibrated with $\mathrm{B}^{+}$buffer before, and washed with W300 (300 mM 
$\mathrm{NaCl}, 10 \mathrm{mM}$ Tris $\mathrm{pH}$ 7.5, $0.5 \mathrm{mM}$ EDTA, $1.5 \mathrm{mM}$ DTT, 1 tablet/50 mL complete EDTA-free protease inhibitor cocktail (ROCHE), $1 \mathrm{mM}$ PMSF, phosphatase inhibitor mix) and W500 buffer (500 mM NaCl, $10 \mathrm{mM}$ Tris pH 7.5, $0.5 \mathrm{mM}$ EDTA, $1.5 \mathrm{mM}$ DTT, 1 tablet/50 mL complete EDTA-free protease inhibitor cocktail (ROCHE), $1 \mathrm{mM}$ PMSF, phosphatase inhibitor mix). Proteins were eluted with $150 \mu \mathrm{L} 0.2 \mathrm{M}$ glycine $\mathrm{pH} 2.5$ and $15 \mu \mathrm{L}$ Tris $\mathrm{pH} 10.4$ and protein concentrations were measured with a NanoDrop ND-1000 photospectrometer. Subsequently, samples were boiled in $3 x$ SDS sample buffer for $10 \mathrm{~min}$ at $95^{\circ} \mathrm{C}$. Samples were stored at $20^{\circ} \mathrm{C}$ until usage.

\subsubsection{Pull downs with HA-tagged fusion proteins}

Protein HA pulldowns were conducted by utilizing Monoclonal Anti-HA-Agarose beads A2095 (SIGMA ALDRICH). A. nidulans strains were inoculated in a concentration of $5^{\star} 10^{8}$ spores in 500 $\mathrm{mL} \mathrm{MM}$ and vegetatively grown for $24 \mathrm{~h}$ in submerged cultures at $37^{\circ} \mathrm{C}$. Mycelia were treated like for GFP trap (see above). HA beads were washed with PBS, equilibrated with $\mathrm{B}^{+}$buffer, added to the filtered supernatant and incubated rotating for $3 \mathrm{~h}$ or $\mathrm{o} / \mathrm{n}$ at $4^{\circ} \mathrm{C}$. The supernatant with the HA beads was loaded onto fresh Poly-Prep ${ }^{\circledR}$ Chromatography Columns (BIO-RAD), which were equilibrated with $\mathrm{B}^{+}$buffer before, and washed with W300 and W500 buffer. Proteins were eluted with $100 \mu \mathrm{L} 0.1 \mathrm{M}$ glycine $\mathrm{pH} 2.5$ and $2.5 \mu \mathrm{L}$ Tris $\mathrm{pH} 10.4$ and protein concentrations were measured with a NanoDrop ND-1000 photospectrometer. Subsequently, samples were boiled in $3 x$ SDS sample buffer for $10 \mathrm{~min}$ at $95^{\circ} \mathrm{C}$. Samples were stored at $-20^{\circ} \mathrm{C}$ until usage. For co-immunoprecipitation to verify protein-protein interactions GFP- and HA pull down experiments were conducted with $A$. nidulans strains harboring GFP- and HAfusion proteins followed by western hybridization

\subsubsection{Bioinformatics analyses of protein features}

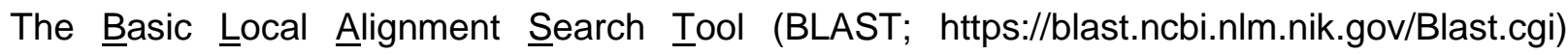
(Altschul et al., 1990) was employed to search for VelB orthologs in other fungi. Pairwise sequence alignments for investigation of VelB orthologs was conducted using EMBOSS Needle (http://www.ebi.ac.uk/Tools/psa/emboss_needle/) (Li et al., 2015; McWilliam et al., 2013; Rice et al., 2000). The Clustal Omega program provided from the European Bioinformatics Institute (EBML-EBI) was employed for multiple sequence alignments (https://www.ebi.ac.uk/Tools/msa/clustalo/) (Li et al., 2015; Sievers et al., 2011). Putative nuclear localization signals were searched with cNLS Mapper (http://nlsmapper.iab.keio.ac.jp/cgi-bin/NLS_Mapper_form.cgi) (Kosugi et al., 2009). The cut-off was set to 5 (score values from 0 to 10). The DISOPRED program was carried out for disorder predictions (http://bioinf.cs.ucl.ac.uk/psipred/) (Ward et al., 2004a). The cut-off was set to 0.5 
(score values from 0 to 1). The DISorder-enhanced $\underline{\mathrm{PHOS}}$ phorylation predictor (DISPHOS) predictor was utilized for phosphosite prediction of the VeIB IDD of $A$. nidulans (http://www.dabi.temple.edu/disphos/) (lakoucheva et al., 2004). The cut-off was set to 0.5 (score values from 0 to 1). The Eukaryotic Linear Motif (ELM) search (http://www.elm.eu.org) (Dinkel et al., 2016) was employed to investigate possible short linear motifs in the VelB IDD of A. nidulans. A cut-off of was set to 0.01 for the probability score was chosen and motif predictions below this threshold are considered to be more likely.

\subsubsection{In vitro protein analyses}

\subsubsection{Recombinant protein expression in E. coli}

The plasmids pETM13-VosA190S, pME3815 and pME4692 were transformed into E. coli Rosetta II (DE3). First, E. coli pre-cultures were inoculated in $50 \mathrm{~mL}$ LB medium supplemented $1: 1000(\mathrm{v} / \mathrm{v})$ with kanamycin and chloramphenicol and incubated $\mathrm{o} / \mathrm{n}$ at $37^{\circ} \mathrm{C}$ on a rotary shaker. The next day, 1 L LB (with kanamycin and chloramphenicol) was inoculated with 15 $\mathrm{mL}$ of the pre-culture and incubated at $37^{\circ} \mathrm{C}$ on a rotary shaker. When an optical density $\mathrm{OD}_{600}$, measured with the BICHROM WPA Biowave DNA Life Science Spectrophotometer (Cambridge, UK), around 0.8 was reached, protein expression was induced with $1 \mathrm{mM}$ IPTG (Isopropyl- $\beta-D$-thiogalactopyranosid) and the cultures were incubated $\mathrm{o} / \mathrm{n}$ at $20^{\circ} \mathrm{C}$ on a rotary shaker. On the following day, the $E$. coli cells were harvested by centrifugation, washed with lysis buffer (30 mM HEPES, $400 \mathrm{mM} \mathrm{NaCl}, 30 \mathrm{mM}$ imidazole) and the cell pellet was stored at $-80^{\circ} \mathrm{C}$ until protein purification.

\subsubsection{Purification of recombinant expressed proteins employing the Äkta Explorer10 system}

The cell pellets were resolved on ice in lysis buffer $(5 \mathrm{~mL}$ per $\mathrm{g}$ of cell pellet) supplemented with $1 \mathrm{mM}$ PMSF (Phenylmethylsulfonylfluorid) $(\mathrm{v} / \mathrm{v})$ to prevent protein degradation. Afterwards, cells were lysed by eight cycles of sonication employing the Sonoplus HD 2070 with the MS72 sonotrode from BANDELIN ELECTRONIC GMBH AND CO. KG (Berlin, Germany). The lysate was cleared by centrifugation in SS34 tubes in the Sorvall RC-5B Plus Refrigerated Centrifuge from THERMO FISHER SCIENTIFIC at $20000 \mathrm{rpm}$ for $30 \mathrm{~min}$ to one hour at $4^{\circ} \mathrm{C}$. Purifications were executed and monitored on the Äkta Explorer10 system from GE HEALTHCARE. The supernatant was applied to two joined $1 \mathrm{~mL}$ HisTrap FF columns (GE HEALTHCARE), which were equilibrated with lysis buffer. After washing, the desired protein was eluted with elution buffer (lysis buffer $+400 \mathrm{mM}$ imidazole) by increasing the concentration of elution buffer step wise about $25 \%$. The eluate was concentrated with Amicon® Ultra 
centrifugal filter with $10 \mathrm{kDa}$ cut-off (MERCK) and subsequently applied to a Sephacryl S-300 16/60 column from GE HEALTHCARE to for size exclusion chromatography. Fractions containing the protein of interest were collected and analyzed by SDS PAGE.

\subsubsection{In vitro co-immunoprecipitation of recombinant expressed proteins}

For in vitro co-IPs, E. coli cells were first inoculated in $5 \mathrm{~mL}$ LB (with kanamycin and chloramphenicol) pre-cultures and incubated $\mathrm{o} / \mathrm{n}$ at $37^{\circ} \mathrm{C}$ on a rotary shaker. The next day, the pre-culture was inoculated in $50 \mathrm{~mL}$ LB (with kanamycin and chloramphenicol) and protein expression was induced with $1 \mathrm{mM}$ IPTG at $\mathrm{OD}_{600}$ of 0.8 . Main cultures were incubated $\mathrm{o} / \mathrm{n}$ at $20^{\circ} \mathrm{C}$ on a rotary shaker. On the following day, cells were harvested by centrifugation at 4000 rpm for $20 \mathrm{~min}$ a $4^{\circ} \mathrm{C}$, washed with buffer $\mathrm{W}(100 \mathrm{mM}$ Tris $\mathrm{pH} 8,150 \mathrm{mM} \mathrm{NaCl}, 1 \mathrm{mM}$ EDTA) and centrifuged again. The pellets were stored at $-80^{\circ} \mathrm{C}$. For protein purification and in vitro coIP the cell pellets were resuspended in buffer W supplemented with $1 \mathrm{mM}$ PMSF and cell lysis was performed by sonication. pETM13-VosAS cells were centrifuged at $20000 \mathrm{rpm}$ for $30 \mathrm{~min}$ at $4^{\circ} \mathrm{C}$. The supernatant was transferred to a $50 \mathrm{~mL}$ reaction tube, $300 \mu \mathrm{L}$ Strep-Tactin ${ }^{\circledR} \mathrm{XT}$ beads from IBA GMBH (Göttingen, Germany) were added and incubated for $3 \mathrm{~h}$ rotating at $4^{\circ} \mathrm{C}$. After incubation the lysate was centrifuged at $1000 \mathrm{rpm}$ for $2 \mathrm{~min}$ at $4^{\circ} \mathrm{C}$ and washed with buffer $\mathrm{W}+1 \mathrm{mM}$ IPTG. The beads were divided into two $15 \mathrm{~mL}$ reaction tubes, the supernatant of lysed pME3815 or pME4692 cells was added and incubated for $2 \mathrm{~h}$ on a rotator at $4^{\circ} \mathrm{C}$. Afterwards, the lysate was centrifuged at $1000 \mathrm{rpm}$ for $2 \mathrm{~min}$ at $4^{\circ} \mathrm{C}$. The beads were washed twice with buffer $\mathrm{W}+1 \mathrm{mM}$ IPTG, transferred to $1.5 \mathrm{~mL}$ reaction tubes ad washed twice again. Subsequently, the supernatant was removed carefully from the beads, $50 \mu \mathrm{L} 3 \mathrm{x}$ SDS sample buffer was added and boiled for $10 \mathrm{~min}$ at $95^{\circ} \mathrm{C}$. The samples were loaded on $12 \%$ SDS gels and on the one hand stained o/n with Coomassie R-250 (40\% (v/v) ethanol, $10 \%(\mathrm{v} / \mathrm{v})$ acetic acid, $0.1 \%(\mathrm{w} / \mathrm{v})$ Coomassie brilliant blue R-250) and to remove the background incubated in Coomassie destaining solution $(12.5 \%(\mathrm{v} / \mathrm{v})$ isopropanol, $10 \%(\mathrm{v} / \mathrm{v})$ acetic acid). Furthermore, western hybridization experiments were performed, for specific detection of His- or Strep-tagged proteins.

\subsection{Identification of proteins form GFP pull downs with mass spectrometry}

\subsubsection{Tryptic protein digestion}

For the whole procedure protein LoBind Tubes PCR Clean EPPENDORF AG (Hamburg, Germany) were used. 
Protein tryptic digestion was performed as described earlier using Sequencing Grade Modified Tryspin (PROMEGA) (Shevchenko et al., 1996). Protein samples were separated according to size on a $12 \%$ SDS gel (see CHAPTER 2.9.2). Subsequently, the gel was incubated in fixing solution (40\% (v/v) ethanol, $10 \%(\mathrm{v} / \mathrm{v})$ acetic acid) for $1 \mathrm{~h}$, washed with $\mathrm{dH}_{2} \mathrm{O}$ and incubated $\mathrm{o} / \mathrm{n}$ in colloidal Coomassie (Kang et al., 2002) (5\% (w/v) aluminum-sulfate-(14-18)-hydrate, 10\% (v/v) methanol, 0.1 (w/v) Coomassie Brilliant Blue G-250, 2\% (v/v) ortho-phosphoric acid). After intensive washing of the stained gel, each lane was excised completely, cut into small pieces of approximately $2 \mathrm{~mm}$, which were shaken in acetonitrile for $10 \mathrm{~min}$ at rt. Subsequently, gel pieces were dried in a SpeedVac Concentrator (THERMO FISHER SCIENTIFIC), which was followed by in-gel reduction by incubating gel pieces in $10 \mathrm{mM}$ DTT in $100 \mathrm{mM} \mathrm{NH}_{4} \mathrm{HCO}_{3}$ for $1 \mathrm{~h}$ at $56^{\circ} \mathrm{C}$. The DTT solution was exchanged by $55 \mathrm{mM}$ iodoacetamide in $100 \mathrm{mM} \mathrm{NH}_{4} \mathrm{HCO}_{3}$ for alkylation of reduced cysteine residues and incubated for $45 \mathrm{~min}$ in the dark at rt. This was followed by washing of gel pieces with $100 \mathrm{mM} \mathrm{NH}_{4} \mathrm{HCO}_{3}$ for $10 \mathrm{~min}$ and dehydration with acetonitrile for $10 \mathrm{~min}$. These steps were done twice while shaking gel pieces at rt. Subsequently, gel pieces were dried in a SpeedVac Concentrator (THERMO FISHER SCIENTIFIC) and afterwards covered with trypsin-digestion buffer (PROMEGA; prepared according to manufacturer's specifications), incubated on ice for $45 \mathrm{~min}$, followed by an incubation in $25 \mathrm{mM} \mathrm{NH}_{4} \mathrm{HCO}_{3}$ o/n at $37^{\circ} \mathrm{C}$. The next day, supernatants were collected after every step into new reaction tubes. After transferring $25 \mathrm{mM} \mathrm{NH}_{4} \mathrm{HCO}_{3}$ from o/n incubation, gel pieces were covered with $20 \mathrm{mM} \mathrm{NH}_{4} \mathrm{HCO}_{3}$ and incubated for 10 min at it on a benchtop shaker for the extraction of acidic peptides. In the next step gel pieces were incubated in $50 \%$ $(\mathrm{v} / \mathrm{v})$ acetonitrile and 5\% (v/v) formic acid and incubated for $20 \mathrm{~min}$ at rt, which was repeated three times. The pooled supernatants were dried completely in a SpeedVac Concentrator (THERMO FISHER SCIENTIFIC). Finally, peptides were resolved in $20 \mu \mathrm{L}$ resuspension buffer $\left(98 \% \mathrm{H}_{2} \mathrm{O}, 2 \%(\mathrm{v} / \mathrm{v})\right.$ acetonitrile, $0.1 \%(\mathrm{v} / \mathrm{v})$ formic acid) and incubated in an ultrasonic bath at maximum power for $3 \mathrm{~min}$ at $35^{\circ} \mathrm{C}$.

\subsubsection{C18 StageTip purification of trypsin-digested samples}

For the whole procedure protein LoBind Tubes PCR Clean EPPENDORF AG (Hamburg, Germany) were used.

Prior to LC-MS/MS measurements, peptides were purified from salts and other contaminants by employing the StageTip purification method (Rappsilber et al., 2003, 2007). For the preparation of $\mathrm{C} 18$ (reverse-phase material) StageTips, C18 plugs were introduced into $200 \mu \mathrm{L}$ pipet tips. StageTips were equilibrated with $100 \mu \mathrm{L}$ of $0.1 \%(\mathrm{v} / \mathrm{v})$ formic acid in HPLC grade methanol, followed by $100 \mu \mathrm{L}$ of $0.1 \%(\mathrm{v} / \mathrm{v})$ formic acid in $70 \%(\mathrm{v} / \mathrm{v})$ acetonitrile and $100 \mu \mathrm{L}$ of $0.1 \%(\mathrm{v} / \mathrm{v})$ formic acid in $\mathrm{dH}_{2} \mathrm{O}$. The last step was repeated. The peptides, which were resolved in resuspension buffer, were loaded onto the equilibrated StageTips and centrifuged at 3500 
rpm for $5 \mathrm{~min}$. This step was repeated and followed by washing the StageTips with $100 \mu \mathrm{L}$ of $0.1 \%(\mathrm{v} / \mathrm{v})$ formic acid in $\mathrm{dH}_{2} \mathrm{O}$ twice. Peptides were eluted with $60 \mu \mathrm{L}$ of $70 \%(\mathrm{v} / \mathrm{v})$ acetonitrile containing $0.1 \%(\mathrm{v} / \mathrm{v})$ formic acid after $5 \mathrm{~min}$ of incubation and subsequently dried completely in in a SpeedVac Concentrator (THERMO FISHER SCIENTIFIC) at $50^{\circ} \mathrm{C}$. Finally, for mass spectrometry, peptides were resolved in $20 \%$ resuspension buffer and incubated in an ultrasonic bath at maximum power for $3 \min$ at $35^{\circ} \mathrm{C}$.

\subsubsection{Identification of proteins by LC-MS/MS2}

Peptide solutions were analyzed with mass spectrometry coupled to liquid chromatography (LC-MS) at the LC-MS facility at the Institute of Microbiology and Genetics. Therefore, an Orbitrap Velos Pro ${ }^{T M}$ mass spectrometer and an Ultimate $3000^{T M}$ liquid chromatography system (both THERMO FISHER SCIENTIFIC) were used. Peptides were separated at nano-flow rates using Acclaim PepMap RSLC ${ }^{T M}$ columns (THERMO FISHER SCIENTIFIC) through a wateracetonitrile gradient. Chromatographically separated peptides were on-line ionized by nanoelectrospray (nESI) using the Nanospray Flex Ion Source ${ }^{T M}$ (THERMO FISHER SCIENTIFIC) at $2.4 \mathrm{kV}$ and continuously transferred into the mass spectrometer. Full scans within the mass range of 300-1850 were recorded with the Orbitrap-FT analyzer at a resolution of 30.000. In parallel parallel data-dependent top-ten fragmentation spectra (MS2) were acquired by collision-induced dissociation (CID) in the LTQ Velos Pro ${ }^{T M}$ linear ion. The XCalibur $2.2^{T M}$ software (THERMO FISHER SCIENTIFIC) was applied for LC-MS method programming and data acquisition. MS/MS2 data processing for protein analysis and identification was carried out with the MaxQuant software and Perseus 1.5.3 software (Cox and Mann, 2008; Tyanova et al., 2016) or the Proteome Discoverer $1.4^{T M}$ software (THERMO FISHER SCIENTIFIC) employing the SequestHT $T^{T M}$ and Mascot $^{T M}$ search engine. As protein database an $A$. nidulans specific database with common contaminants was used.

\subsection{Spore viability assay}

The analyzation of viability of conidiospores was conducted as described earlier ( $\mathrm{Ni}$ and $\mathrm{Yu}$, 2007; Sarikaya-Bayram et al., 2010). Conidiospores were harvested after two days and counted with a Coulter Z2 particle counter (BECKMANN). Subsequently, $1^{*} 10^{5}$ spores we plated on solid MM, harvested after 2, 5 and $10 \mathrm{~d}$ of growth in the light at $37^{\circ} \mathrm{C}$ and 200 spores were spread on solid MM plates, which were grown in the light at $37^{\circ} \mathrm{C}$. After $2 \mathrm{~d}$ the formation of upcoming colonies was counted and calculated as ratio of the number of growing colonies to the number of inoculated spores. This test was performed in triplicates per experimental day. 


\section{$2.12 \quad$ Fluorescence microscopy}

Fluorescence microscopy was conducted with a Zeiss AxioObserver Z.1 inverted confocal microscope, equipped with Plan-Neofluar 63x/0.75 (air), Plan-Apochromat 63x/1.4 oil and a Plan-Apochromat 100x/1.4 oil objectives (ZEISS) and a QuantEM:512SC camera (PHOTOMETRICS, Tucson, AZ, USA). Pictures were processed with the SlideBook 6.0 software package (INTELLIGENT IMAGING INNOVATIONS GMBH, Göttingen, Germany).

For fluorescence microscopy 2000 spores per strain were inoculated in 8-well borosilicate cover glass system (THERMO FISHER SCIENTIFIC) in $400 \mu \mathrm{L}$ liquid MM for vegetative growth and grown for $18 \mathrm{~h}$ at $30^{\circ} \mathrm{C}$. Fluorescence values of wildtype background fluorescence were subtracted from strains expressing GFP-fusion proteins to allow for normalization of fungal auto fluorescence. Nuclei were visualized via staining with $0.1 \%(\mathrm{w} / \mathrm{v}) 4^{\prime}, 6^{\prime}$-diamidino-2phenylindole (DAPI) (ROTH) and incubation for $10 \mathrm{~min}$ at rt prior to microscopy. 


\section{Results}

\subsection{The intrinsically disordered domain of $A$. nidulans VelB contains an $\mathrm{N}$-terminal motif conserved among ascomycetes}

Four velvet domain proteins, namely $\mathrm{VeA}$, VelB, VelC and VosA, are described in A. nidulans (Park et al., 2017). This protein family is characterized by the conserved velvet domain, which is a fungal specific DNA-binding and dimerization domain and comprises around 150 amino acids (FIGURE 4) (Bayram and Braus, 2012; Gerke and Braus, 2014). The velvet domain did not show significant sequence similarity to any known protein domain, but the crystal structure of the velvet domain of $A$. nidulans VosA revealed structural similarities to the Rel homology domain of the mammalian transcription factor NF-KB (FIGURE 5) (Ahmed et al., 2013). In the VelB-VosA $A_{1-190}$ heterodimer crystal structure, no structural information could be gained for 99 amino acids in the middle of the VelB velvet domain (FIGURE 6). This region has presumably been removed by protease treatment during crystallization. Protein structure prediction revealed that this protein sequence is unstructured (Ahmed et al., 2013) and in the following is denominated as intrinsically disordered domain (IDD). VelB is the only protein in the family of velvet regulatory proteins with this interruption, whereas VeA, VelC or VosA, possess a continuous velvet domain (FIGURE 4) (Gerke and Braus, 2014).

The amino acid sequence of the velvet domain of VelB is highly conserved among filamentous fungi (FIGURE 13, red boxes) and it was investigated in silico if this is also the case for the IDD of VelB (FIGURE 13, yellow box). Multiple sequence alignments were carried out employing the Clustal Omega program provided by the European Bioinformatics Institute (EBML-EBI) (Li et al., 2015; Sievers et al., 2011). The amino acid sequence of the IDD is conserved among Aspergilli (FIGURE 13). 


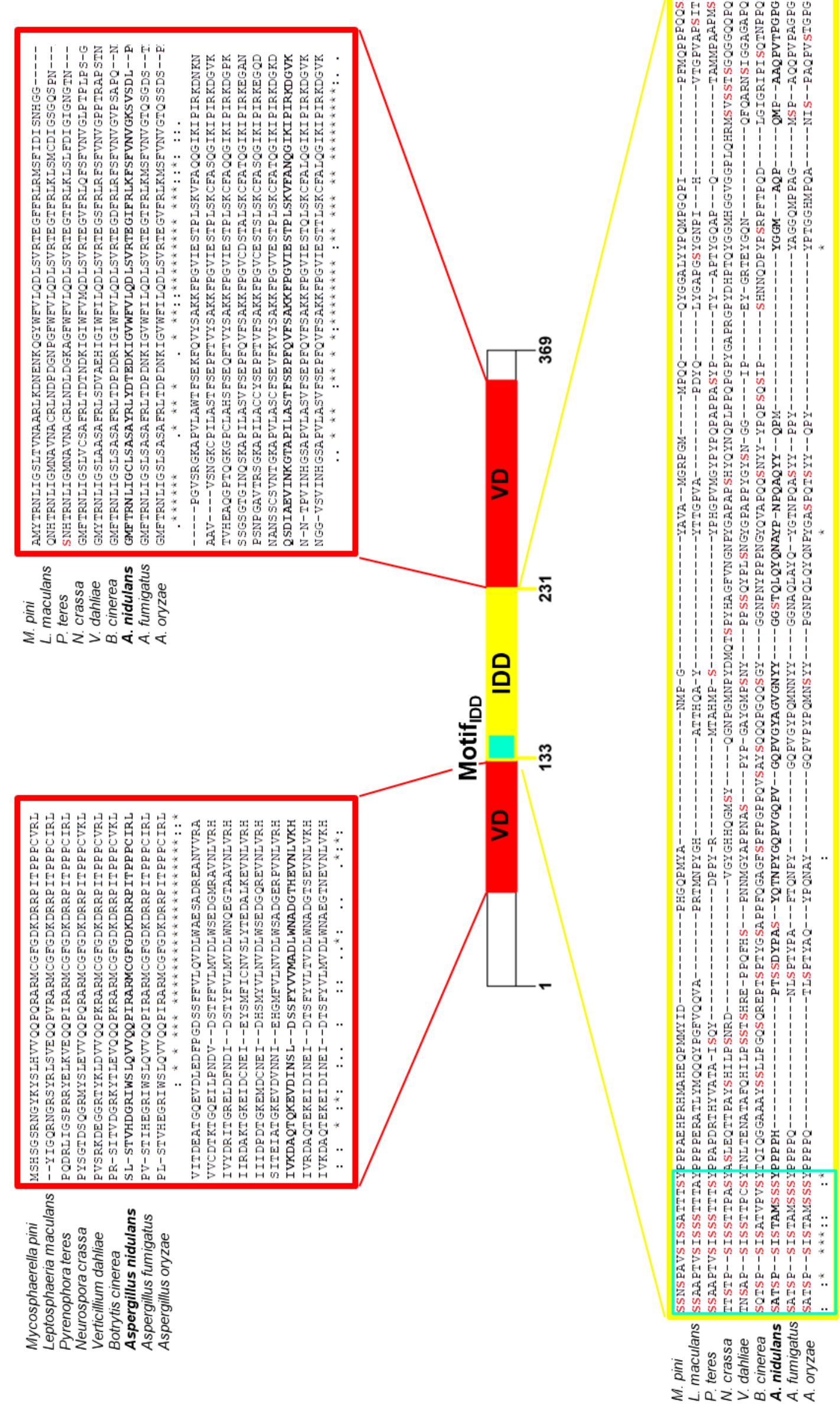


FIGURE 13: Ascomycetes share a conserved motif in the VelB intrinsically disordered domain.

Multiple amino acid sequence alignments of $A$. nidulans VelB (bold letters) with VelB orthologs from other ascomycetes. The velvet domain (VD, red box) of VelB is interrupted by a 99 amino acid intrinsically disordered domain (IDD) (yellow box, residue 133-231 in A. nidulans), which contains a conserved N-terminal motif (Motif $f_{\mid D D}$, turquoise box) in ascomycetes. Serine residues (S) in the IDD are highlighted in red. Gene IDs of VelB orthologs: Mycosphaerella pini: DOTSEDRAFT_139168, Leptosphaeria maculans: LEMA_P063930.1, Pyrenophora teres: PTT_16366-1, Neurospora crassa: NCU02775, Verticillium dahliae: VDAG08715, Botrytis cinerea: BofuT4P246000014001, Aspergillus nidulans: AN0363. Aspergillus fumigatus: Afu1g09170, Aspergillus oryzae: AO090005000898.

VelB orthologs in other ascomycetes and basidiomycetes were found with the help of the Basic Local Alignment Search Iool (BLAST) (FIGURE 13, FIGURE 14) (Altschul et al., 1990). The velvet domain of VelB orthologs in other filamentous fungi is also interrupted, but the sequences of the IDD differ considerably. Ascomycetes like Mycosphaerella pini, Leptosphaeria maculans, Pyrenophora teres, Neurospora crassa, Verticillium dahliae and Botrytis cinerea share a serine-rich conserved 11 amino acid motif (Motif IDD) $_{\text {) }}$ at the N-terminus of the IDD (FIGURE 13, turquoise box). The velvet domains of the VelB orthologs in basidiomycetes like Coprinopsis cinerea, Laccaria bicolor or Cryptococcus neoformans are also interrupted, and even the Motif $f_{\mathrm{IDD}}$, which is conserved in the analyzed ascomycetes, is absent (FIGURE 14).

Taken together, the interruption of the VelB velvet domain is conserved in the fungal kingdom but its sequence, in contrast to the one of the velvet domain, evolved rapidly. Aspergilli still share a similar amino acid sequence of the VelB IDD, whereas in other ascomycetes only few residues at the $\mathrm{N}$-terminus are conserved and in basidiomycetes the amino acid sequence evolved considerably. 


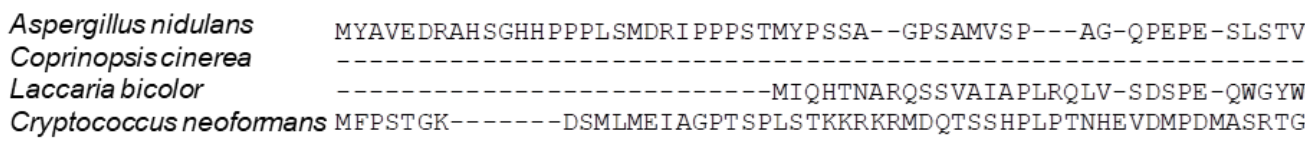

Aspergillus nidulans HDGRIWSLQVVQQPIRARMCGFGDKDRRPITPPPCIRLIVKDAQTQKEVD INSLDSSFYV Coprinopsiscinerea Laccaria bicolor MIVRT -EDQKLVDVDDVDCSF FI LGRKHYSLEVVQH PLRARMCGF GDKDRRPLAPAAVAKMVVRR -DDNS IVDVDDMDCSF FI Cryptococcus neoformans REGWTYHLE ILQE PLRARACGFGNKDRRPLTPPP I IRLWIQDA-LGNQVD PNIVDSNT I I : : :

Aspergillus nidulans Coprinopsiscinerea Laccaria bicolor

Cryptococcus neofomans

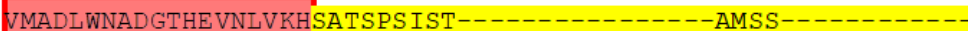
VTVDLWSAD GKQEMNLVLHPS STDKYAPPNPPTKPSASTTRRR-TISSSSRPPGHQA-SN TVDLWSED GKHE TNLVLH SS VDRH I PPHSSTKSRRRGTSS S-A--PQSRAPANOTPSG QVDLCSADGHEGRNVVRH VVGPGSVPAVVSVSENVRS GHVD PSTLPTTS TAVSEQPYRN

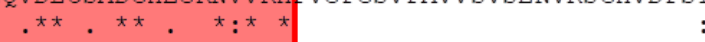

Aspergillus nidulans Coprinopsiscinerea Laccaria bicolor ------------------------------- SYPP PPHP ------- TSSDYP

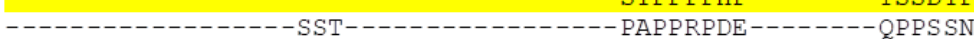
--------------GSTPTL------SQHRPGEQRPPLPHP-------HASGYN Cryptococcus neoformans S SMDYSSWPEQYSSESSDRTTPGWTYQDAFVSSPRIAGTRPPMARRVRTP TRPSTAPS LR

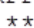

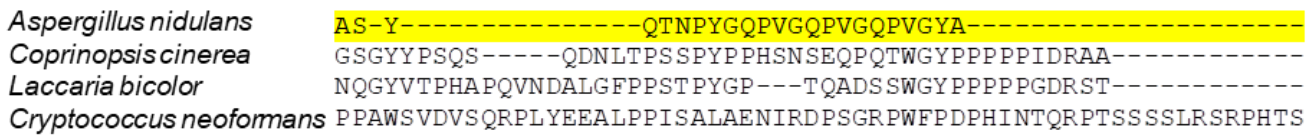

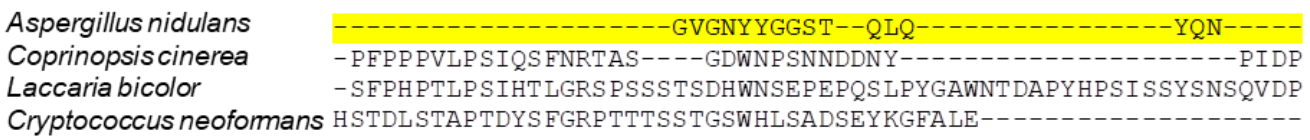

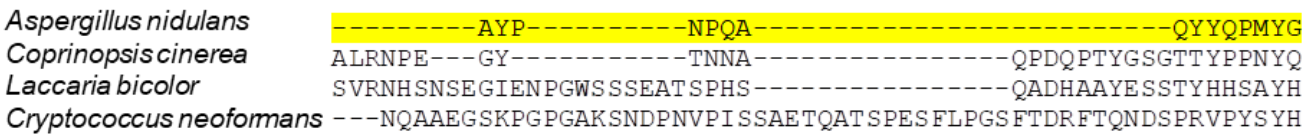
$\begin{array}{ll}\text { Aspergillus nidulans } & \text { GMA-QPQM------PAA--------QPVTPGPGGMFTRNL IGCLSASAYRLYDTEDKI } \\ \text { Coprinopsiscinerea } & \text { TP----PMP PNSGN-AT P--------QNN I PRNIATYTRTLVGPLSANACRLIDEHRKP } \\ \text { Laccaria bicolor } & \text { PAPTHSQMAPGTCSSQHV--------STVPPPP HTYTRTLVGPLSANACRLLDEHRKP }\end{array}$ Cryptococcus neoformans NSHYRSDVAPGKERDSFHSPDLDTMKETRCFAPA SVASTLVLVGKRHTPCNKLKDEHGRI

Aspergillus nidulans Coprinopsiscinerea Laccaria bicolor

Cryptococcus neofomans

GVWFVLQDL SVRTEGIFRLKFS FVNV GKSVSDLPQSDIAEVINKGTAPILASTF SEPFQV ¿IFFL FQDLSVRTEGTFRLRMRLMNVGAPPAPEPG---SCRVHNDI SPILAQTETEPF IV I FFL EQDL SVRTEGTFRLRMRLMNVGAPPAPEVG---AARVHNDV SPVLAQTETEQETV

Aspergillus nidulans Coprinopsiscinerea Laccaria bicolor

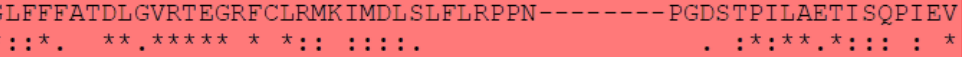

Cryptococcus neoformans

ESAKK FPGVIEST PLSKVFANQGIKI PIRKDGVKGQGSRRH SDEDDGLD--NEY-Y SAKR F PGV PDTTALS I AFGNQGQKL PLRNRHGT GSKRRRRNQSGS EGES EDDS --SAKR FPGV PDTTALS IALGNQGQKLPLASDGL SEALVEES SR--VQQASETQWFRV YSAKR FPGV IPTTKLTRLFAAQGIKLAVRE SHKQ KHRS KENVDMDVQDEI EEDE DGQ

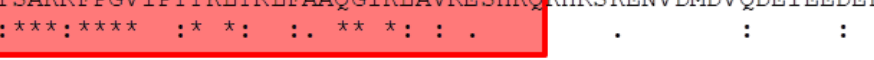

FIGURE 14: The amino acid sequence of the A. nidulans VelB IDD differs from the IDD of representatives of the Basidiomycota.

Multiple amino acid sequence alignments of Aspergillus nidulans VelB compared to deduced sequences of basidiomycetes orthologs. The highly conserved velvet domain is marked in red and the IDD of $A$. nidulans VelB is highlighted in yellow. Gene IDs: Coprinopsis cinerea: CC1G_01332, Laccaria bicolor: LACBIDRAFT_292785, Cryptococcus neoformans: 105460.t01. 


\subsection{The VelB intrinsically disordered domain supports selective heterodimer formation with VosA in A. nidulans}

Velvet domain proteins form different homo- and heterodimers, bind to certain target genes and regulate transcription in response to extra- and intracellular signals (Ahmed et al., 2013; Bayram et al., 2008). Proteins, which are intrinsically disordered can function as hubs in protein interaction networks (Haynes et al., 2006; Ota et al., 2016; Patil et al., 2010). They play a central role in protein-protein and protein-nucleic acid interactions, thereby controlling regulation of crucial cellular processes such as the subcellular localization transcription or signaling networks (Dunker et al., 2005; Liu et al., 2006; Mao et al., 2013; Wright and Dyson, 2015).

\subsubsection{The subcellular localization of VeIB is independent of the IDD}

VelB is localized in the cytoplasm and in the nucleus independent of light (Bayram et al., 2008). A strain expressing a version of VelB that lacks the IDD, fused to sGFP (VelB $\left.{ }^{I D D}{ }_{-} G F P\right)$ and another with the full length VelB fused to sGFP (VelB-GFP) were generated to examine the role of the VelB IDD for localization and heterodimer formation. Respective genetic fusion constructs were transformed in locus by homologous recombination in both cases and genes were expressed under the control of the native promoter of velB.

The subcellular localization of VelB with or without the IDD was compared and fluorescence microscopy revealed that the VelB ${ }^{I D D} \Delta$-GFP and the VelB-GFP fusion proteins are localized in the cytoplasm as well as in nuclei of vegetative growing hyphae (FIGURE 15). Constitutively expressed GFP and the wildtype strain served as controls to exclude unspecific or background GFP signals. In conclusion, the subcellular distribution of VelB between the cytoplasm and the nucleus does not depend on the IDD. This suggests that, as VelB does not possess an NLS, the VeIB IDD is dispensable for the interaction with proteins, which enable its shuttle into the nucleus. 


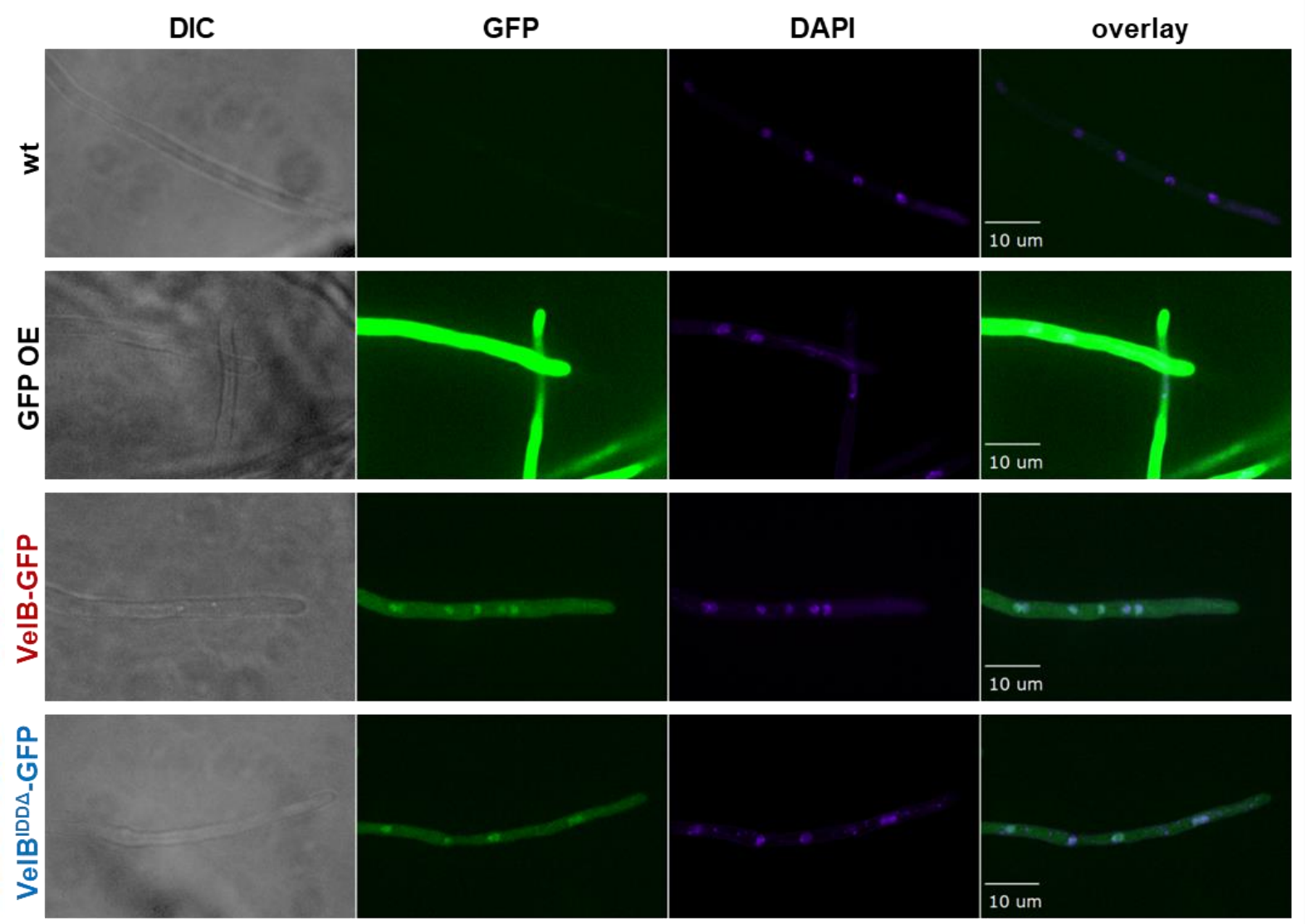

FIGURE 15: The VelB subcellular localization is independent of the IDD in A. nidulans.

VelB-GFP with or without the IDD is localized in the cytoplasm and accumulates in the nuclei of hyphae. Nuclei were visualized with DAPI (4',6-diamidin-2-phenylindol). Strains were vegetatively grown in submerged cultures for 18 hours at $30^{\circ} \mathrm{C}$. wt $=$ wildtype, $\mathrm{OE}=$ over expression, $\mathrm{DIC}=$ differential interference contrast, scale bar $=10 \mu \mathrm{m}$.

\subsubsection{VeA-VelB heterodimer formation is independent of the IDD}

VelB ${ }^{\mathrm{IDD}} \Delta_{-}$GFP and VelB-GFP are both localized in the cytoplasm and accumulate in nuclei of hyphae, suggesting a shuttle of VelB independent of the IDD. VeA is known to enhance the transport of VelB from the cytoplasm into the nucleus in the dark (Bayram et al., 2008). Besides the interaction with VeA, VelB also forms a heterodimer with VosA in the nucleus (Sarikaya-Bayram et al., 2010). GFP pull down experiments were conducted to further investigate whether VelB interacts with $\mathrm{VeA}$ and VosA in the absence of the IDD and to examine and compare the interactome of VelB with and without the IDD. VelB protein is detectable in high amounts in western hybridization experiments during vegetative growth (Park et al., 2012b), which is supported by the strong GFP signal in fluorescence microscopy experiments (FIGURE 15). Therefore, mycelium from submerged cultures was used for GFP pull downs followed by LC-MS/MS for the identification of putative interacting proteins. Three proteins were concordantly identified for both, VelB-GFP and VelB ${ }^{I D D}-$ GFP pull downs (FIGURE 16), namely the bait protein (VelB-GFP or VelB ${ }^{I D D} \Delta-G F P$, respectively), CatB and the velvet domain protein VeA (TABLE 7). This confirms that the IDD is dispensable for the formation of 
the VelB-VeA complex. VeA can bind to and enhance the shuttle of VelB into the nucleus independently of the IDD. In contrast to this commonality, the GFP pull downs revealed several differences regarding the putative interaction partners of VelB with and without the IDD. The bait proteins VelB-GFP and VelB ${ }^{\mathrm{IDD}} \Delta_{-}$GFP were detected with similar LFQ intensities and MS/MS counts. Furthermore, comparable numbers of unique peptides were identified, indicating that both pull downs worked equally well. 32 putative interaction partners were found for VelB ${ }^{I D D} \Delta_{-}$GFP, which were not detected in the GFP pull downs using the full length VelBGFP as bait. These proteins were sorted and grouped according to their predicted cellular function or localization: translation, primary metabolism, RNA maturation and processing, membrane/cell wall, signaling, cell compartments, DNA binding and unknown function (FIGURE 16, TABLE 7).

One protein was exclusively identified for VelB-GFP pull down experiments, which was not found to interact with VelB ${ }^{I D D}$-GFP (FIGURE 16). This protein is the velvet domain protein VosA (TABLE 7), which acts as repressor of asexual development and regulator of sporogenesis (CHAPTER 1.5.2).

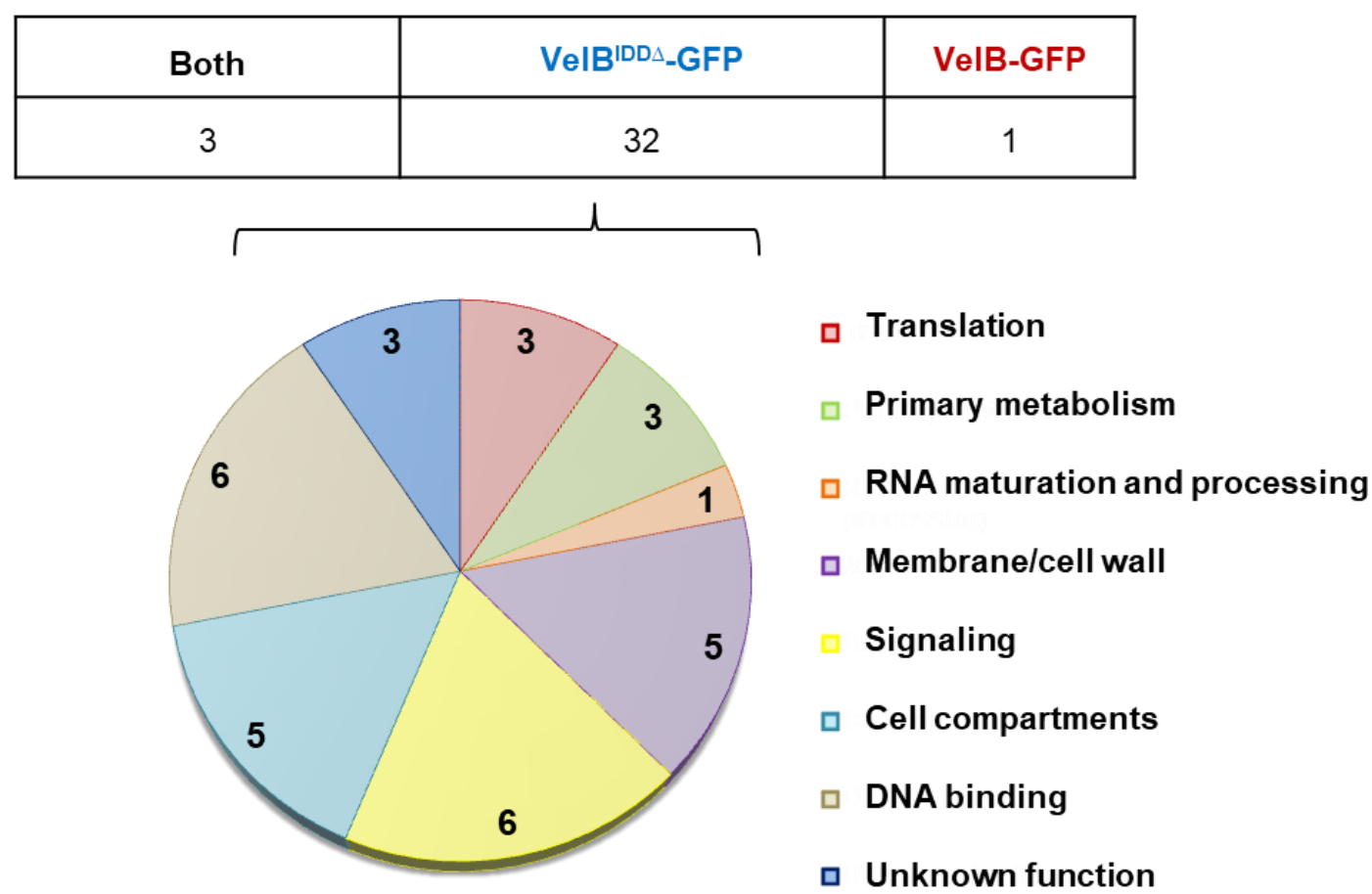

FIGURE 16: The VelB IDD alters selective heterodimer formation.

GFP pull downs of VelB-GFP and VelB ${ }^{\mathrm{IDD}} \Delta_{\text {-GFP }}$ from vegetatively grown cultures followed by LC-MS/MS revealed that the lack of the IDD leads to increased number of putative interaction partners (TABLE 7). The diagram depicts the predicted cellular function or localization of the 32 putative interaction partners of VelB ${ }^{I D D} \Delta_{-}$GFP. Numbers represent proteins identified in at least two out of three biological repetitions with MS/MS counts $\geq 4$, unique peptides $\geq 3$ and LFQ intensity $\geq 20$ and which were absent in the control strain. 
VelB interacts with $\mathrm{VeA}$ in both, cytoplasm and nucleus, and interaction with VosA is proposed to occur exclusively in the nucleus (Sarikaya-Bayram et al., 2010). VelB ${ }^{\operatorname{DDD}}{ }_{-G}$-GFP pulled down VeA, but not VosA, and instead 32 other proteins (TABLE 7). These proteins were examined for putative nuclear localization signals by employing the cNLS Mapper program (Kosugi et al., 2009) to investigate possible competitors of VeA or shared interaction partners, which might also be able to bind to VeIB in the nucleus. For almost half of the putative interaction partners (14 proteins) a nuclear localization signal with a score $\leq 5$ was predicted (TABLE 7 , underlined AN numbers). This score was chosen as these proteins presumably can shuttle between, and be localized in both, the cytoplasm and the nucleus. Interesting candidates will be further discussed in CHAPTER 4.3.1.

TABLE 7: Putative interaction partners identified in the GFP pull down of VelB-GFP and VelB ${ }^{\mathrm{IDD}} \Delta$-GFP.

Proteins were identified in at least two out of three biological repetitions with MS/MS counts $\geq 4$, unique peptides $\geq 3$ and LFQ intensity $\geq 20$ and which were absent in the control strain. Proteins with underlined AN numbers contain a nuclear localization signal predicted with cNLS mapper (Kosugi et al., 2009) with a score $\leq 5$ (indicating a localization in both, nucleus and cytoplasm), Sys. Name = systematic name, Std. name = standard name, unchar. = uncharacterized. Descriptions from AspGD are given (Cerqueira et al., 2014).

\begin{tabular}{|c|c|c|c|c|}
\hline $\begin{array}{l}\text { Sys. } \\
\text { Name }\end{array}$ & $\begin{array}{l}\text { Std. } \\
\text { name }\end{array}$ & Description & $\underline{\text { VelB }^{\operatorname{IDD} \Delta}}$ & $\underline{\text { VelB }}$ \\
\hline AN1959 & VosA & $\begin{array}{l}\text { Nuclear protein involved in spore formation and } \\
\text { trehalose accumulation }\end{array}$ & - & + \\
\hline AN0363 & VelB & Bait protein & + & + \\
\hline AN1052 & VeA & $\begin{array}{l}\text { Protein involved in light-sensitive control of } \\
\text { differentiation and secondary metabolism }\end{array}$ & + & + \\
\hline AN9339 & CatB & $\begin{array}{l}\text { Hyphal catalase with a predicted role in } \\
\text { gluconic acid and gluconate metabolism }\end{array}$ & + & + \\
\hline \multicolumn{5}{|c|}{ Translation } \\
\hline AN0907 & unchar. & $\begin{array}{l}\text { Putative } 40 \text { S ribosomal protein S23 (Rps23), } \\
\text { putative ortholog of } S \text {. cerevisiae RPS23A }\end{array}$ & + & - \\
\hline AN1345 & unchar. & $\begin{array}{l}\text { Putative } 40 \text { S ribosomal protein S22 (Rps22), } \\
\text { putative ortholog of } S \text {. cerevisiae RPS22A }\end{array}$ & + & - \\
\hline AN0314 & unchar. & $\begin{array}{l}\text { Putative aspartyl-tRNA synthetase, putative } \\
\text { ortholog of S. cerevisiae DPS1 }\end{array}$ & + & - \\
\hline
\end{tabular}




\begin{tabular}{|l|l|l|c|c|}
\hline \multicolumn{2}{|l|}{ Primary metabolism } \\
\hline AN5226 & AcpA & Acetate permease $\underline{\text { A, involved in acetate uptake }}$ & + & - \\
\hline AN9180 & unchar. & $\begin{array}{l}\text { Putative transketolase, putative ortholog of } \\
\text { A. fumigatus TktA }\end{array}$ & + & - \\
\hline AN1967 & PpoA & $\begin{array}{l}\text { Psi factor producing oxygenase } \text { A, responsible } \\
\text { for the formation of the oxylipin psiBa }\end{array}$ & + & - \\
\hline
\end{tabular}

\section{RNA maturation and processing}

\begin{tabular}{|l|l|l|l|l|}
\hline AN7474 & unchar. & $\begin{array}{l}\text { Has domain(s) with predicted RNA binding, } \\
\text { putative ortholog of } \text { S. cerevisiae JSN1 }\end{array}$ & + & - \\
\hline
\end{tabular}

\section{Membrane/cell wall}

\begin{tabular}{|c|c|c|c|c|}
\hline AN5020 & $\begin{array}{l}\text { ArfB/ } \\
\text { AdpA }\end{array}$ & $\begin{array}{l}\text { ADP ribosylation factor } \underline{B} \text {, required for normal } \\
\text { endocytosis and polarized growth }\end{array}$ & + & - \\
\hline AN9079 & unchar. & $\begin{array}{l}\text { Putative ortholog of } N \text {. crassa Ham-10, with role } \\
\text { in conidia formation }\end{array}$ & + & - \\
\hline AN2928 & unchar. & Putative cell wall protein, uncharacterized & + & - \\
\hline AN11139 & AbpA & $\begin{array}{l}\text { Putative actin-binding protein } \underline{A} \text { of the cortical } \\
\text { actin patches }\end{array}$ & + & - \\
\hline AN3098 & NsfA & $\begin{array}{l}\text { Putative secretory component, ortholog of } \\
\text { S. cerevisiae SEC } 18 \text {, has similarity to } \\
\text { mammalian } \underline{N} \text {-ethylmaleimide-sensitive factor }\end{array}$ & + & - \\
\hline
\end{tabular}

\section{Signaling}

\begin{tabular}{|l|l|l|c|c|}
\hline AN3102 & PhkA & $\begin{array}{l}\text { Putative histidine-containing phosphotransfer } \\
\text { protein }\end{array}$ & + & - \\
\hline$\underline{\text { AN3422 }}$ & Ste7 & $\begin{array}{l}\text { MAP kinase kinase (MAPKK), component of a } \\
\text { signaling module SteD-SteC-MkkB-MpkB that } \\
\text { controls coordination of development and } \\
\text { secondary metabolism }\end{array}$ & + & - \\
\hline$\underline{\text { AN2130 }}$ & unchar. & $\begin{array}{l}\text { Putative Ras guanine-nucleotide exchange } \\
\text { factor activity, putative ortholog of S. cerevisiae } \\
\text { CDC25 }\end{array}$ & + & - \\
\hline$\underline{\text { AN7576 }}$ & unchar. & $\begin{array}{l}\text { Predicted Rho GTPase activating protein, } \\
\text { putative ortholog of S. pombe Rga1 }\end{array}$ & + \\
\hline
\end{tabular}




\begin{tabular}{|c|c|c|c|c|}
\hline AN8836 & Cla4 & $\begin{array}{l}\text { Predicted PAK (p21-activated kinase) family } \\
\text { protein }\end{array}$ & + & - \\
\hline AN0463 & unchar. & $\begin{array}{l}\text { Predicted Rac guanine nucleotide exchange } \\
\text { factor, putative ortholog of } C \text {. albicans DCK2 }\end{array}$ & + & - \\
\hline \multicolumn{5}{|c|}{ Cell compartments } \\
\hline AN4207 & unchar. & $\begin{array}{l}\text { Putative ortholog of } C \text {. albicans APL4, with role } \\
\text { in endosomal transport, vesicle mediated } \\
\text { transport and AP-1 adaptor complex }\end{array}$ & + & - \\
\hline AN0995 & unchar. & $\begin{array}{l}\text { Putative CLASP family microtubule-associated } \\
\text { protein, putative ortholog of } S . \text { pombe Peg1 }\end{array}$ & + & - \\
\hline AN3029 & unchar. & $\begin{array}{l}\text { Putative AP-1 adaptor complex subunit beta, } \\
\text { Putative ortholog of } C \text {. albicans APL2 }\end{array}$ & + & - \\
\hline AN0706 & unchar. & $\begin{array}{l}\text { Ortholog(s) have role in ER to Golgi vesicle- } \\
\text { mediated transport, putative ortholog of } \\
\text { C. albicans USO1 }\end{array}$ & + & - \\
\hline AN4168 & unchar. & $\begin{array}{l}\text { DUF500 and SH3 domain protein, putative } \\
\text { ortholog of S. pombe actin cortical patch } \\
\text { component Lsb4 }\end{array}$ & + & - \\
\hline \multicolumn{5}{|c|}{ DNA binding } \\
\hline AN0228 & unchar. & $\begin{array}{l}\text { Putative ortholog of S. cerevisiae replication } \\
\text { licensing factor MCM6 }\end{array}$ & + & - \\
\hline AN6070 & unchar. & $\begin{array}{l}\text { Putative ortholog of S. cerevisiae replication } \\
\text { licensing factor MCM4 }\end{array}$ & + & - \\
\hline AN2278 & unchar. & $\begin{array}{l}\text { Putative ortholog of } N \text {. crassa SNF2-family ATP } \\
\text { dependent chromatin remodeling factor Snf21 } \\
\text { (Crf3-1) }\end{array}$ & + & - \\
\hline AN4187 & unchar. & $\begin{array}{l}\text { Putative ortholog of } N \text {. crassa TBP associated } \\
\text { factor (Cfr8-1) }\end{array}$ & + & - \\
\hline AN7222 & unchar. & NACHT domain containing protein & + & - \\
\hline AN5168 & unchar. & $\begin{array}{l}\text { Putative NACHT and Ankyrin domain protein, } \\
\text { putative ortholog of } S \text {. pombe Akr1 }\end{array}$ & + & - \\
\hline
\end{tabular}




\begin{tabular}{|l|l|l|c|c|}
\hline AN3005 & unchar. & Protein of unknown function & + & - \\
\hline AN11181 & unchar. & Protein of unknown function & + & - \\
\hline AN5423 & unchar. & Protein of unknown function & + & - \\
\hline
\end{tabular}

Taken together, VelB with and without IDD can interact with VeA, which is presumably necessary to support the transfer from the cytoplasm to the nucleus. In contrast, VosA was only identified in pull downs with VelB-GFP, but not with VelB ${ }^{\mathrm{IDD}} \Delta_{-} \mathrm{GFP}$, indicating that it might not interact with VelB ${ }^{\mathrm{IDD}}$-GFP. These findings suggest that the IDD, which is dispensable for VelB-VeA heterodimer formation, is specifically necessary to allow the formation of the VelB-VosA heterodimer.

\subsubsection{The IDD is required for stable VelB-VosA heterodimer formation}

VosA was not identified to interact with VelB ${ }^{\operatorname{IDD}}{ }_{\text {-GFP }}$ in GFP pull down experiments of vegetatively grown cultures. Co-immunoprecipitation (Co-IP) experiments were conducted to verify these findings. vosA::ha and velB::sgfp or velB ${ }^{I D D \Delta}:: s g f p$ fusion constructs were transformed in locus and GFP and HA pull down experiments were carried out. Subsequently, western hybridization experiments were performed for the specific detection of the fusion proteins. Employment of the $\alpha-\mathrm{HA}$ antibody revealed a strong signal, depicting the VosA-HA protein on the western membrane. This was detected only for the pull down of the full length VelB-GFP fusion protein (FIGURE 17A, violet arrow). Application of the $\alpha$-GFP antibody revealed a $13 \mathrm{kDa}$ shift between VelB-GFP (70 kDa, red arrow) and VelB ${ }^{\mathrm{IDD}} \Delta_{-} \mathrm{GFP}$ (57 kDa, blue arrow), reflecting the 99 amino acids missing in the VelB ${ }^{\mathrm{IDD} \Delta}$-GFP fusion protein (FIGURE 17A). Signal intensities of protein bands from western hybridization experiments of three biological replicates were quantified employing the image processing Fiji software (Schindelin et al., 2012). This quantification revealed a ten-fold increased amount of the VosA signal relative to the amount of VelB or VelB ${ }^{I D D}$, respectively. The reciprocal experiment resulted in the identification of VosA-HA in equal amounts for both HA pull downs (FIGURE 17B, violet arrow). Application of the a-GFP antibody resulted in the recognition of the full length VelB-GFP (FIGURE 17B, red arrow). VelB ${ }^{I D D} \Delta_{-}$GFP was not detected in three biological replicates. These findings corroborate that the IDD is necessary for a stable and specific VelB heterodimer formation with VosA in A. nidulans during vegetative development. 
A

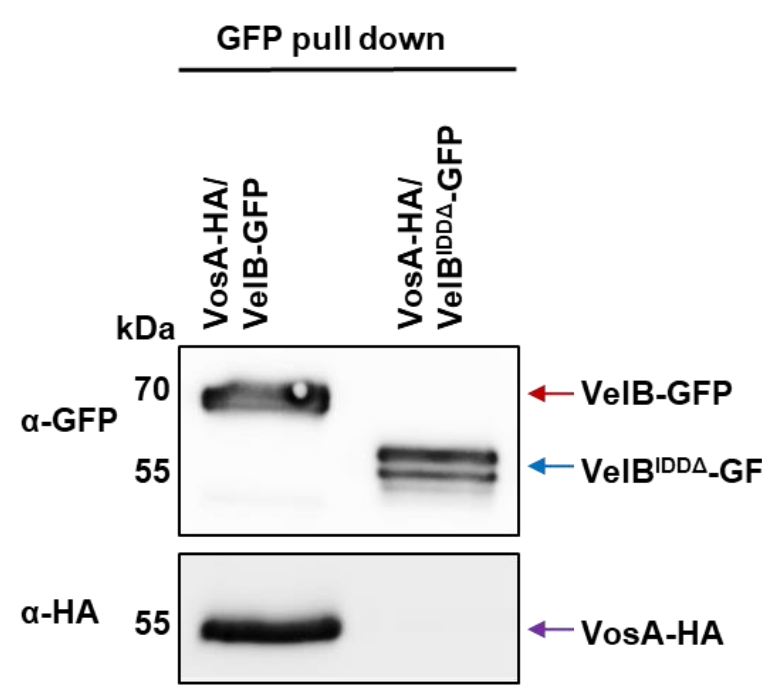

B

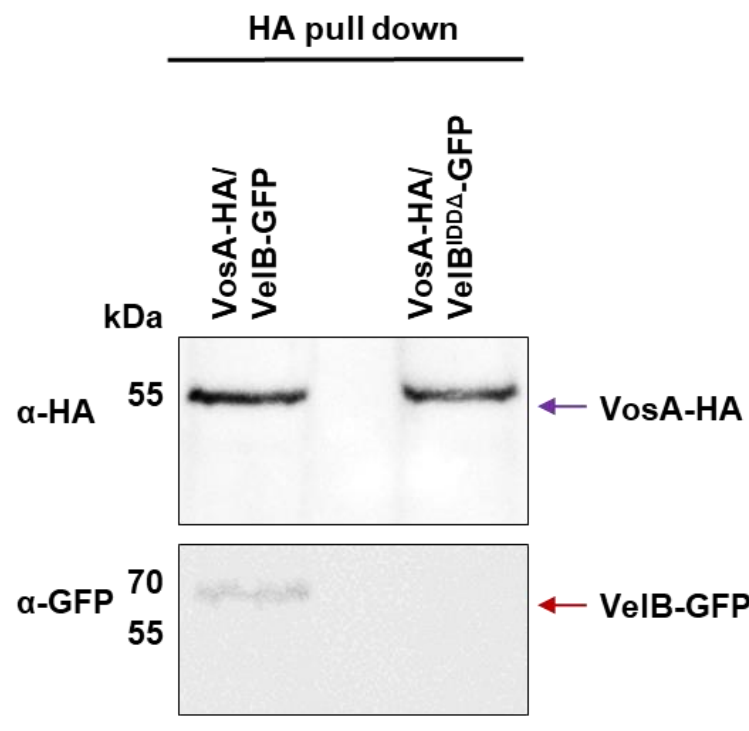

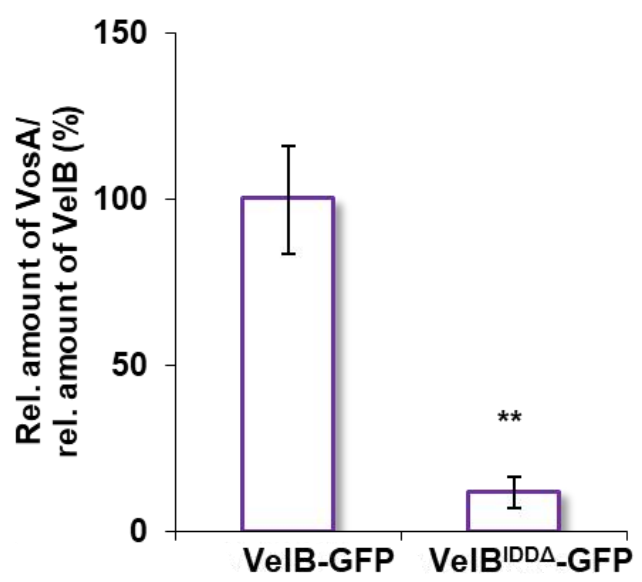

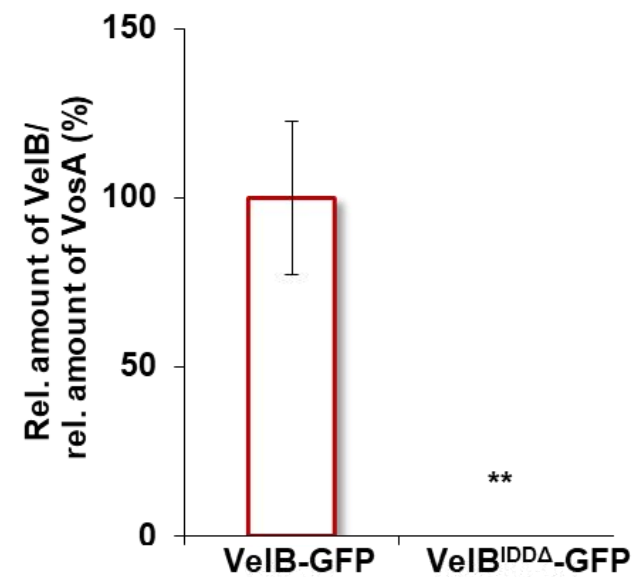

FIGURE 17: The VelB IDD is required for co-immunoprecipitation of the VelB-VosA heterodimer.

GFP pull downs (A) and HA pulldown experiments (B) of strains expressing VosA-HA and VelB-GFP or VelB ${ }^{\mathrm{IDD}} \Delta_{\text {-GFP }}$ fusion proteins followed by western hybridization with GFP or HA antibody were performed. The relative amount of VosA against the relative amount of VelB (violet diagram) or the relative amount of VelB against the relative amount of VosA (red diagram) was quantified. Error bars indicate standard error of the mean (SEM) of three biological replicates. $p$-value was calculated with standard deviation. ${ }^{* *} ; p<0.01$. 


\subsubsection{The VelB IDD is required for VelB-VosA heterodimer formation in vivo but not in vitro}

The VelB IDD is required for VelB-VosA heterodimer formation but the VelB IDD is not present in the crystal structure of the A. nidulans VelB-VosA $A_{1-190}$ heterodimer (Ahmed et al., 2013). In the work from Ahmed and co-workers a truncated version of VosA encompassing residue 1-190 (Vos $\left.A_{1-190}\right)$, representing the velvet domain, was cloned, expressed and purified to increase the chance for crystallization (Ahmed et al., 2013). In the case of VelB the full length protein was used for complex formation and crystallization with $\operatorname{Vos}_{1-190}$ (Ahmed et al., 2013). In the present study, it was investigated whether the VeIB IDD is required for heterodimer formation with the velvet domain of VosA in vitro. Co-immunoprecipitation experiments with Vos $A_{1-190}$-Strep and VelB-His or VelB ${ }^{I D D} \Delta_{-} H$ is fusion proteins, recombinantly expressed in E. coli, were performed in vitro. This was conducted to examine, whether the short Vos $\mathrm{A}_{1-190}$ protein interacts with VelB when the IDD is deleted before complex formation. The VosA $_{1-190}$-Strep fusion protein appears as a band at $25 \mathrm{kDa}$, which was visualized with the $\alpha$-Strep antibody. The presence of VelB-His $(41 \mathrm{kDa})$ and $\mathrm{VelB}^{\mathrm{IDD} \Delta}-\mathrm{His}(30 \mathrm{kDa})$ was confirmed with the $\alpha$-His antibody. VelB-His (FIGURE 18, red arrow) and VelB ${ }^{I D D}{ }_{-}-H i s$ (blue arrow) were both detected to interact with the short $\operatorname{Vos}_{1-190}$-Strep protein (violet arrow). This confirms that the intrinsically disordered domain of VelB is dispensable for the interaction of VelB with the shortened VosA $\mathrm{A}_{1-190}$ in vitro. The C-terminal part of VosA might be therefore contributory to the selective heterodimer formation of VelB-VosA in the cell in vivo. Taken together, these data suggest that heterodimerization is possible in vitro. In contrast, the interaction of an additional protein(s) to the VeIB IDD or modification by posttranslational modification might trigger the decision whether the VeIB-VosA heterodimer is formed in vivo. 


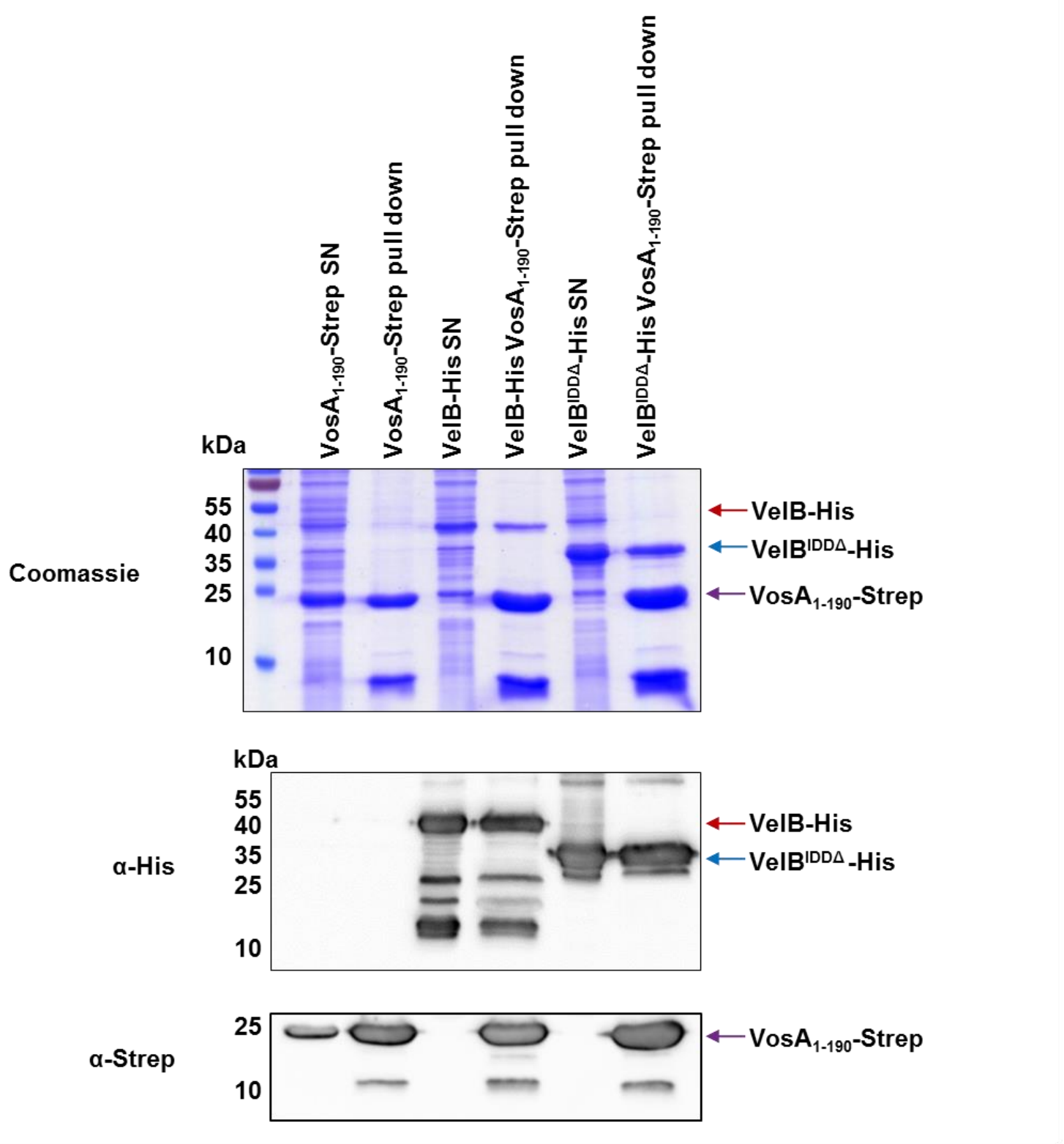

FIGURE 18: VelB binds to the velvet domain of VosA in vitro independently of the IDD.

In vitro co-immunoprecipitation of the velvet domain of VosA (VosA $\left.{ }^{1-190}-S t r e p\right)$ and VelB-His or $V_{\text {VelB }}{ }^{\mathrm{IDD}}$-His fusion proteins recombinantly expressed in $E$. coli. Protein expression and purification was visualized on a SDS polyacrylamide gel stained with Coomassie. Specific detection of fusion proteins was achieved with $\alpha$-His or $\alpha$-Strep antibodies. Pull down experiments employing Streptactin beads were conducted. Vos $\mathrm{A}_{1-190}$-Strep is the bait protein (violet arrow) and pulls VelB-His (red arrow) and VelB ${ }^{\operatorname{IDD} \Delta}$-His (blue arrow) fusion proteins. $\mathrm{SN}=$ supernatant of lysed cells after centrifugation. The membrane shown here was incubated with $\alpha$-His followed by $\alpha$-mouse and subsequently stripped and incubated with $\alpha$-Strep, followed by $\alpha$-mouse. The figure showing the signal detected with $\alpha$-Strep, does not depict the whole membrane, as stripping did not completely remove $\alpha$-His bands at 35 and $40 \mathrm{kDa}$. 


\subsubsection{The VelB IDD is necessary for homodimer formation in vitro}

The IDD is required for VelB-VosA heterodimer formation but dispensable for the interaction between VelB and VeA. Besides heterodimer formation with VeA or VosA, VelB can also form homodimers (Sarikaya-Bayram et al., 2010). Therefore, the role of the IDD regarding homodimer formation was investigated. VelB-His or VelB ${ }^{I D D} \Delta_{-}-H i s$ fusion proteins were recombinantly expressed in E. coli to analyze the influence of the IDD on dimerization with itself. Both proteins were purified by affinity chromatography (FIGURE 19) followed by size exclusion chromatography (FIGURE 20) to differentiate between monomeric and dimeric VelB$\mathrm{His} / \mathrm{VeIB}^{\mathrm{IDD} \Delta}$-His. First, VelB-His or VelB ${ }^{\mathrm{IDD} \Delta}-\mathrm{His}$ fusion proteins were pre-purified on columns packed with $\mathrm{Ni}^{2+}$ Sepharose beads. Unspecific proteins, which did not bind to the $\mathrm{Ni}^{2+}$ beads, eluted immediately (FIGURE 19, black arrow). Concentration of the elution buffer, containing imidazole to replace the His-fusion proteins on the beads, was increased stepwise (FIGURE 19, green line). The addition of $25 \%$ elution buffer (FIGURE 19, green arrow) was enough to wash the desired protein from the column.

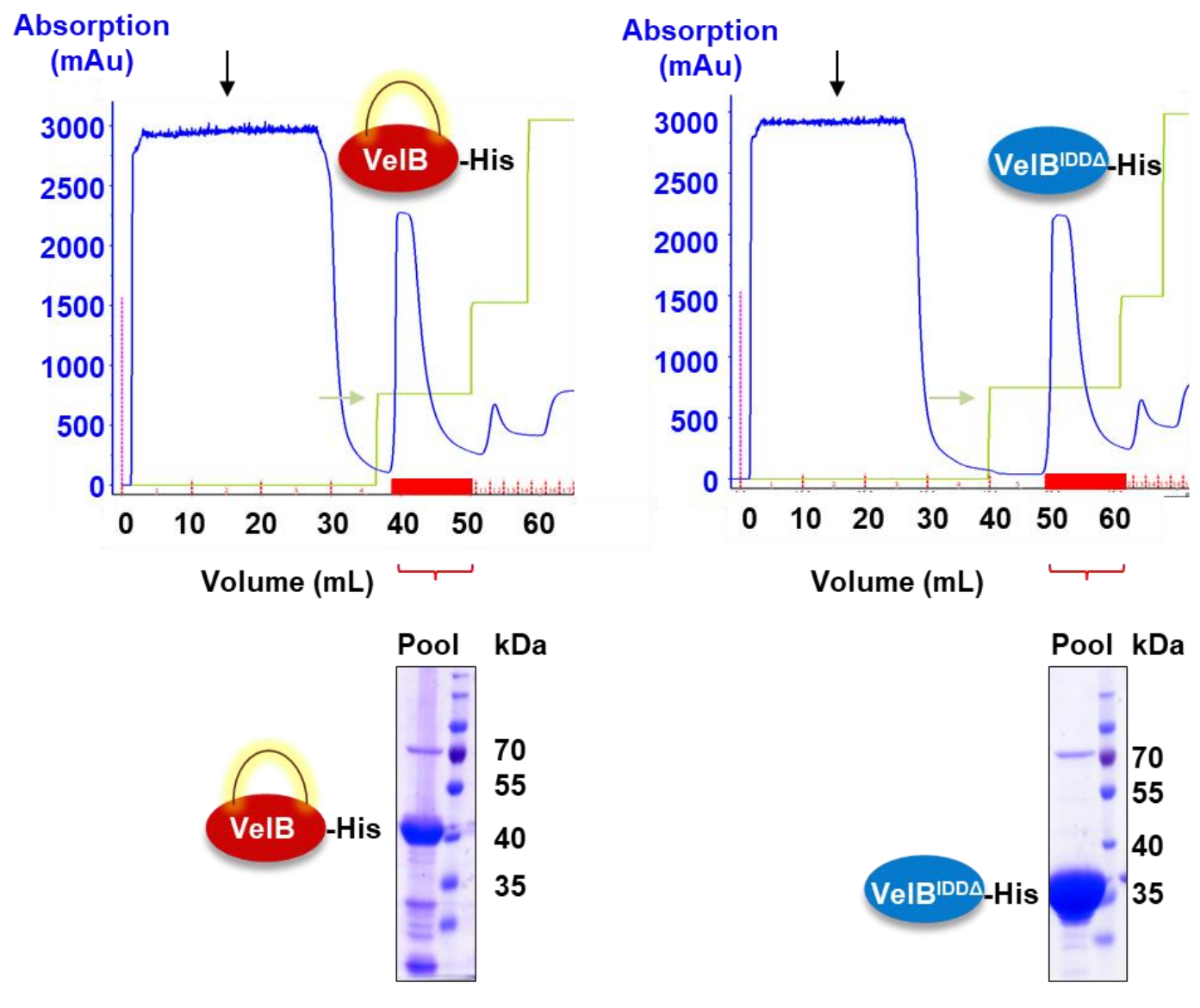

FIGURE 19: Affinity chromatography of VelB-His and VelB ${ }^{I D D} \Delta-H i s$ fusion proteins.

VelB-His and VelB ${ }^{\mathrm{IDD} \Delta_{-}}$His fusion proteins were purified using HisTrap FF columns. Black arrows $=$ unspecifically bound proteins, green arrow $=25 \%$ increase in concentration of elution buffer. Red line and brackets show the elution of the protein of interest. The fractions containing the protein were pooled and visualized by SDS PAGE on a $10 \%$ acrylamide gel. 
The fractions containing the purified proteins (FIGURE 19, red bar) were pooled and further purified by size exclusion chromatography. Two UV maxima were detected for VelB-His in this experiment (FIGURE 20A, run I). The maximum at $62.75 \mathrm{~mL}$ corresponds to the molecular weight of the VelB-His homodimer and the second UV maximum eluting after $73.5 \mathrm{~mL}$ corresponds to the monomeric VelB-His. VelB-His from fractions of both peaks was visualized on a polyacrylamide gel through SDS PAGE (FIGURE 20B, red arrow). In contrast, VelB ${ }^{\text {IDD } \Delta_{-} \text {His }}$ is eluting in one peak (FIGURE 20A, run II) which elution volume of $74.23 \mathrm{~mL}$ corresponds to the monomeric protein. The molecular weight of VelB ${ }^{\mathrm{IDD} \Delta_{-}}$His was confirmed with SDS PAGE (FIGURE 20C, blue arrow).

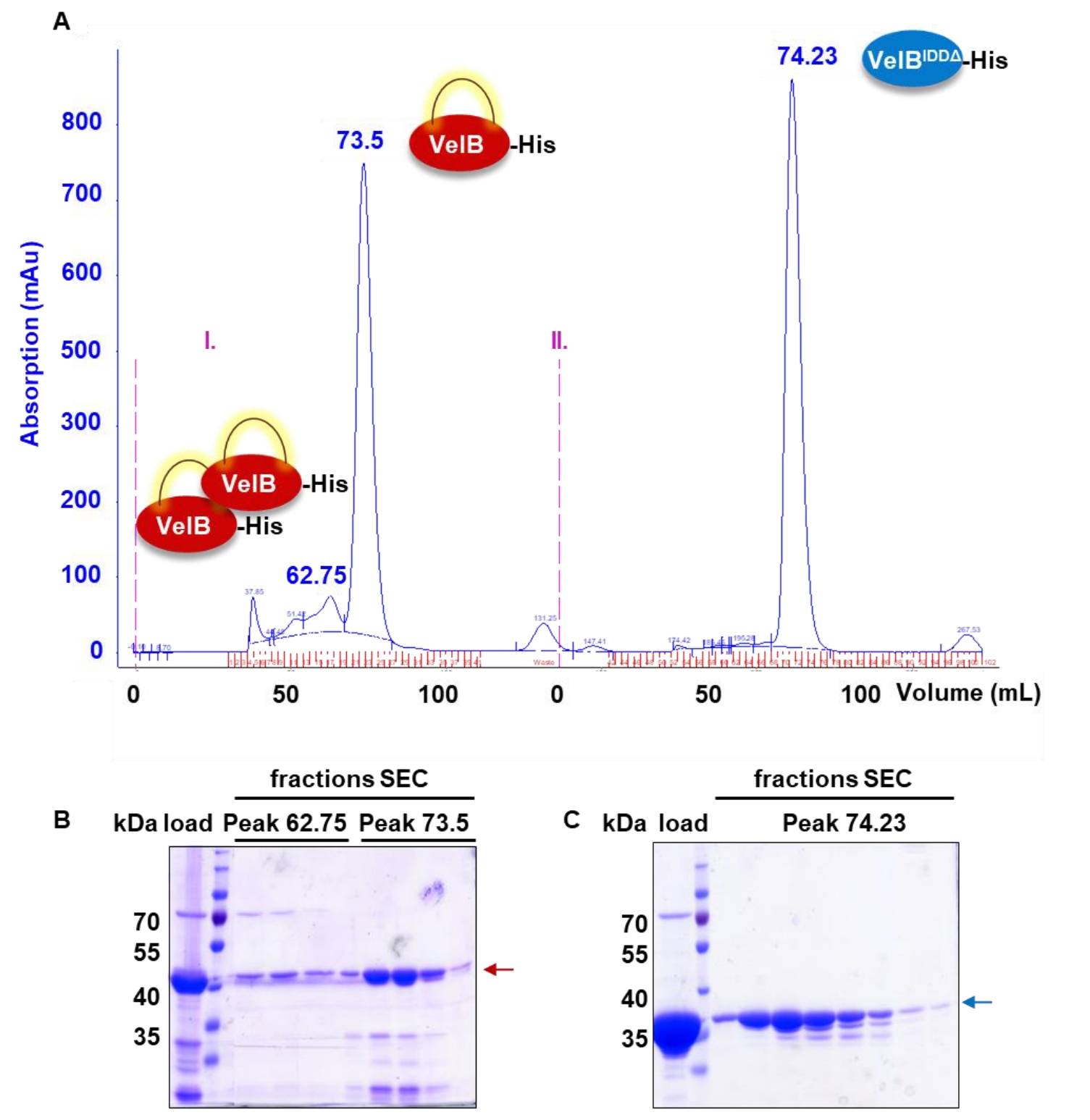

FIGURE 20: Homodimer formation of VelB is dependent on the IDD in vitro.

Size exclusion chromatography shows two runs of the full length VelB-His (I.) and the VelB ${ }^{I D D} \Delta_{-} H i s$ (II.) fusion protein. The full length VelB-His elutes as dimer and monomer. VelB ${ }^{\mathrm{IDD}} \Delta_{-}$His elutes only as a monomer. $\mathrm{B}$. and $\mathrm{C}$. Proteins of the eluates were visualized on a $10 \%$ SDS gel and stained with Coomassie (red arrow $=$ VelB-His, blue arrow $=\operatorname{VelB}^{\mathrm{IDD} \Delta_{-}}-\mathrm{His}$ ). 
Taken together, these data suggest that a VelB without the intrinsically disordered domain is exclusively able to interact with one velvet domain protein, namely $\mathrm{VeA}$, during vegetative growth. This indicates that in the absence of the VeIB IDD, the VelB-VeA heterodimer can be formed, which supports nuclear import. Specificity with other velvet domain proteins like a second VelB for the VelB-VelB homodimer or VosA for the VelB-VosA heterodimer requires the VeIB IDD and possibly additional interacting cellular control proteins.

\subsection{The IDD of VelB is required for efficient asexual spore formation in}

\section{A. nidulans}

Velvet domain proteins coordinate development and secondary metabolism in A. nidulans by forming dimers and the heterotrimeric velvet complex VelB-VeA-LaeA (Bayram et al., 2008). These complexes bind to and regulate the expression of certain target genes. Deletion of velvet protein encoding genes leads to imbalances in dimer formation, resulting in misregulation of developmental programs. The deletion of the velB gene prevents VelB-VeA heterodimer formation, leading to the complete loss of sexual fruiting body formation (Bayram et al., 2008). Furthermore, the lack of velB (velBA) reduces the viability of spores and conidiospore production is reduced and delayed (Park et al., 2012b). The VeIB IDD is required for selective heterodimer formation. It was analyzed whether this influences development in $A$. nidulans.

The growth and differentiation of the vel$B^{I D_{\Delta}}$ mutant was investigated and compared to wildtype and velB $\Delta$ strains during light conditions promoting asexual development resulting in the production of conidiospores (FIGURE 21, FIGURE 22). Formation of conidiospores with two different initial situations was investigated. The spores were inoculated as a single point in the middle of the plate (FIGURE 21) or distributed over the whole agar plate (FIGURE 22). Point inoculation results in the radial growth from a single spot. After three days of growth the $v e l B^{I D D_{\Delta}}$ colony is not as green as the wildtype colony, but after seven days no difference is visible between these strains anymore. That suggests that, the VeIB IDD is dispensable for an accurate early light response and conidiophore formation in young growing hyphae is just slightly changed in the velB $B^{I D D_{\Delta}}$ mutant. 


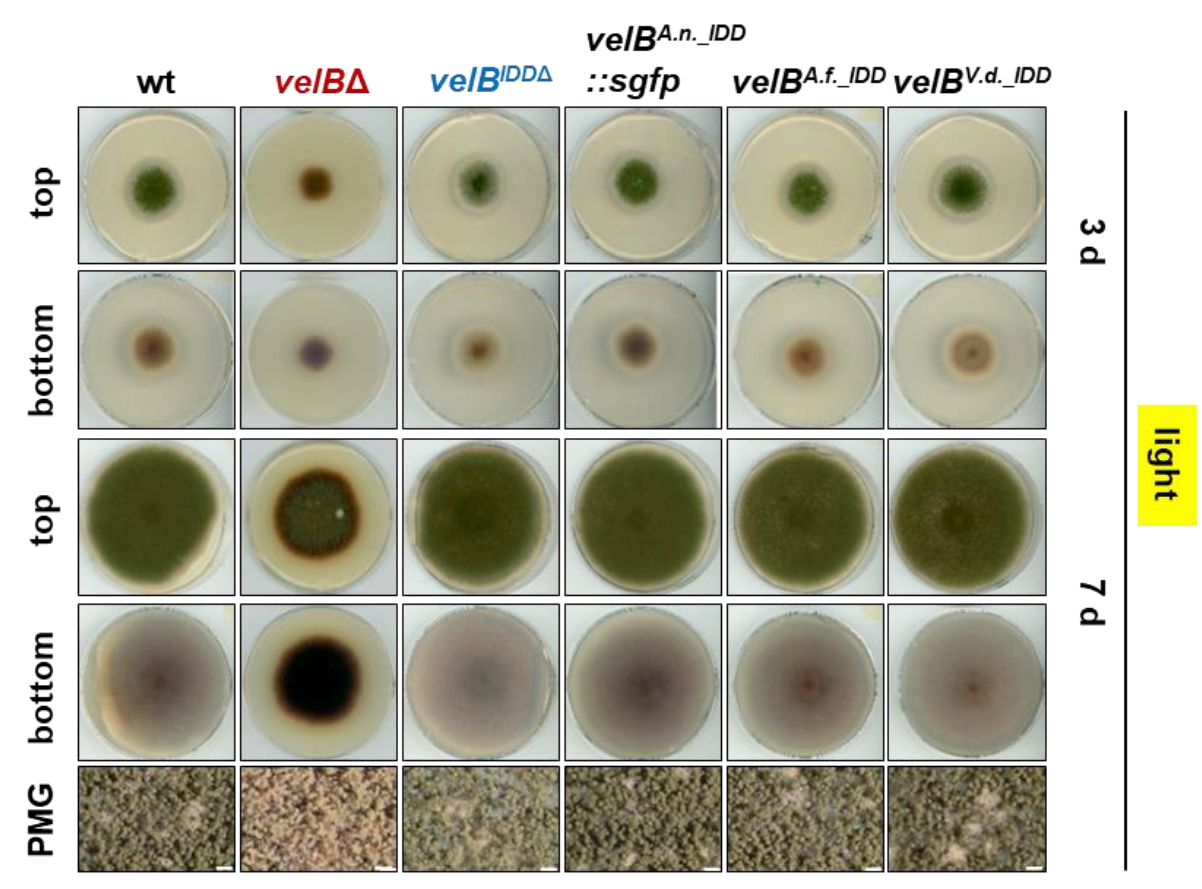

FIGURE 21: The VelB IDD is dispensable for conidiospore formation in hyphae growing from a single spot in $A$. nidulans.

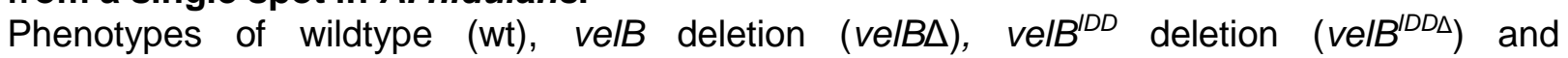
complementation strains with the IDD of $A$. nidulans, $A$. fumigatus or Verticillium dahliae into

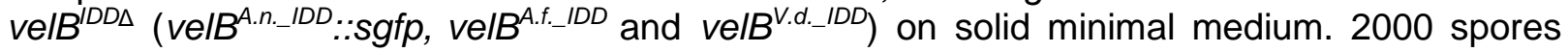
were spotted in the middle of the plate and grown in light for 3 or 7 days (d) at $37^{\circ} \mathrm{C}$. PMG $=$ photomicrograph, scale bar (white) $=100 \mu \mathrm{m}$.

In contrast, conidiospore formation is decreased in the velB $B^{I D D_{\Delta}}$ mutant, when the spores are distributed over the whole plate. The vel$B^{I D D_{\Delta}}$ mutant has a white fluffy appearance, due to the formation of increased amounts of aerial hyphae (FIGURE 22A, orange arrow). Conidiophores with conidiospores are formed at the edge of the agar plate (FIGURE 22A, black arrow). Quantification of plated conidiospores from FIGURE 22A during growth in light revealed reduced conidiospore formation of $12 \%$ after three and $14 \%$ after seven days relative to wildtype (FIGURE 22B). This indicates that a high spore density requires the IDD and therefore presumably VelB-VosA or VelB-VelB for accurate conidiation and conidiophore formation within the mycelium. In contrast, conidiophore formation from a single spot and light control requires the VelB-VeA heterodimer and not the VelB-VosA or VelB-VelB complex.

The velB deletion mutant secretes red-brown pigments into the agar, and the bottom of the plates appears much darker than the one from the wildtype. In contrast the agar of the $v e / B^{I D D_{\Delta}}$ mutant has a lighter color than the wildtype (FIGURE 21, FIGURE 22). This implies alteration in the secondary metabolism of $A$. nidulans. The intrinsically disordered domains of the ascomycetes $A$. fumigatus and Verticillium dahliae were chosen for complementation experiments. Pairwise sequence alignments employing EMBOSS Needle (Li et al., 2015; McWilliam et al., 2013; Rice et al., 2000) revealed a $60.8 \%$ sequence similarity of the VelB 
IDDs of $A$. nidulans and $A$. fumigatus, whereas $A$. nidulans and $V$. dahliae show just $27.7 \%$ sequence similarity. As it was shown in the alignment, these three species share the conserved Motif

A

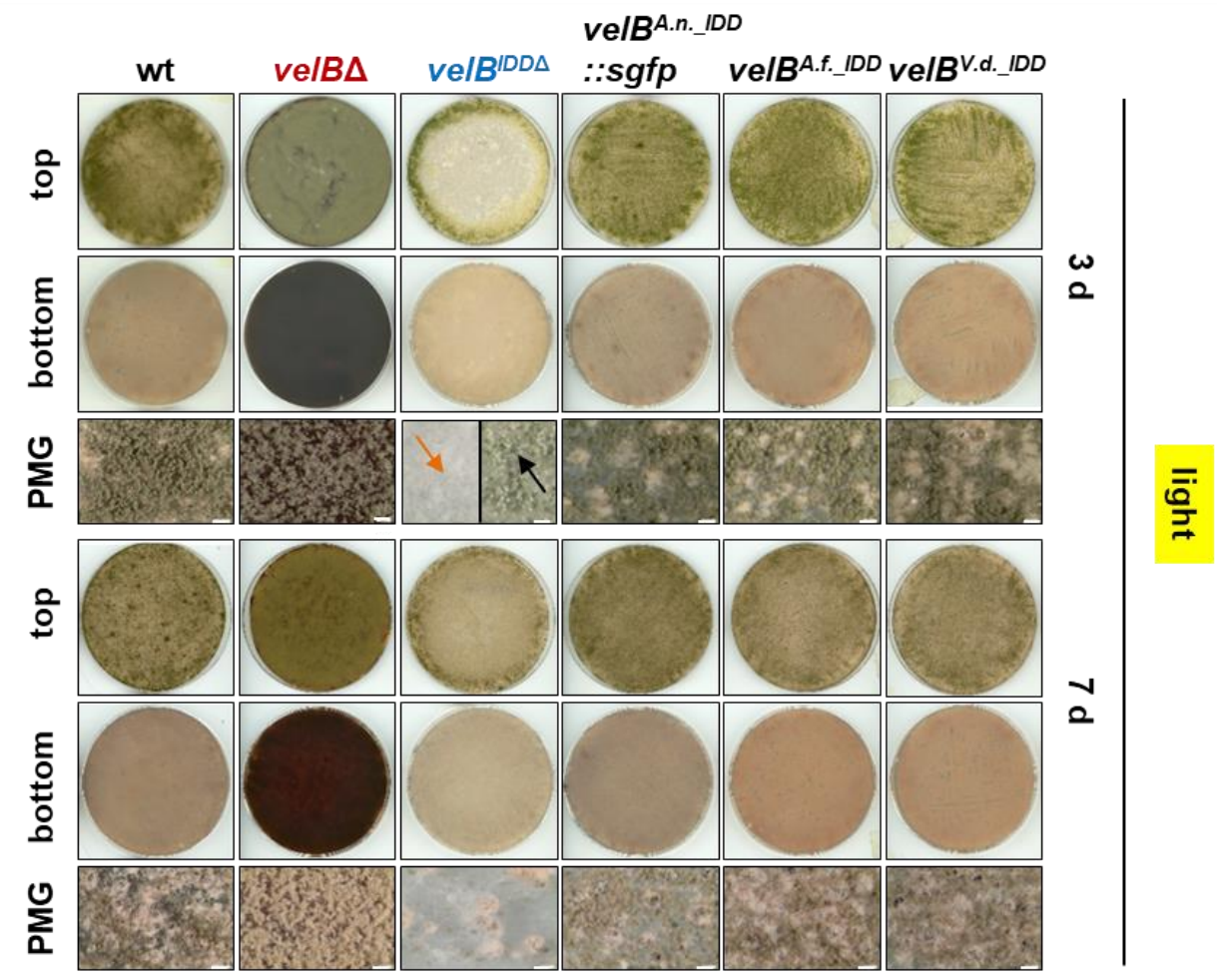

B

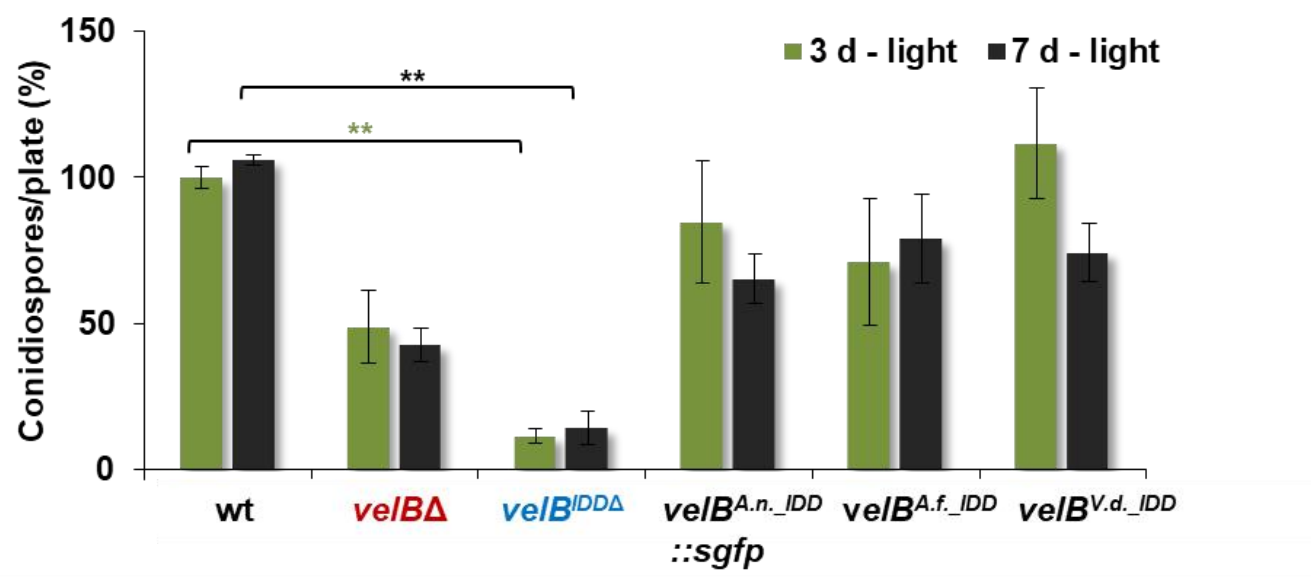

FIGURE 22: The IDD of VelB is required for efficient asexual spore formation within aging mycelium in $A$. nidulans.

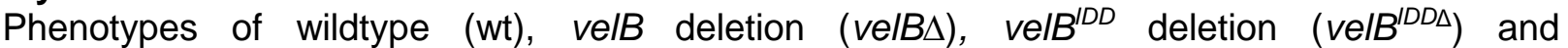
complementation with the IDD of $A$. nidulans, $A$. fumigatus or Verticillium dahliae into vel $B^{I D D \Delta}$

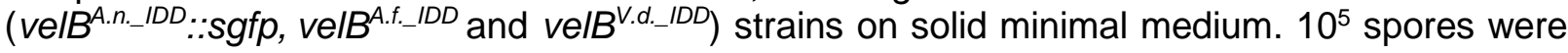
distributed over the plate and grown in light for 3 or 7 days (d) at $37^{\circ} \mathrm{C}$. $P M G=$ photomicrograph, scale bar (white) $=100 \mu \mathrm{m}$. B. Quantification of conidiospores from strains shown in $A$. The vel $B^{I D D \Delta}$ mutant produces after three days $12 \%$ conidiospores compared to wildtype. Error bars indicate standard error of the mean (SEM) of three biological replicates. $p$ value was calculated with standard deviation. ${ }^{* *}, p<0.01 ;{ }^{* * *}, p<0.005$. 
The velB $B^{I D D_{\Delta}}$ mutant phenotype could be rescued by the (in locus) introduction of the IDD of $A$. nidulans, $A$. fumigatus or Verticillium dahliae into the vel$B^{I D D_{\Delta}}$ strain, indicating functional conservation of the IDD of $A$. nidulans, $A$. fumigatus and $V$. dahliae.

$A$. nidulans mainly reproduces sexually through the formation of sexual ascospores-producing cleistothecia when grown in the dark and with limited oxygen supply. The velB deletion mutant is completely abolished to reproduce sexually, even in the dark (FIGURE 23). The velB ${ }^{I D D_{\Delta}}$ mutant was analyzed regarding sexual development and the capability to form mature fruiting bodies after seven days of growth in the dark. Therefore, spores of the wildtype, velB $\Delta$, $v e / B^{I D D_{\Delta}}$ and the complemented strains were spotted on minimal medium and incubated for three and seven days in the dark. Sexual development is not altered in the vel$B^{I D D_{\Delta}}$ mutant compared to wildtype and after seven days black mature cleistothecia, filled with ascospores, are formed (FIGURE 23, black arrows). Furthermore, the complementation with the IDDs of $A$. fumigatus and $V$. dahliae did not change the phenotype of $A$. nidulans compared to wildtype when grown in light or dark. This indicates that these IDDs do not disturb within the VelB velvet domain but fully complement the velB ${ }^{I D D_{\Delta}}$ mutant phenotype.

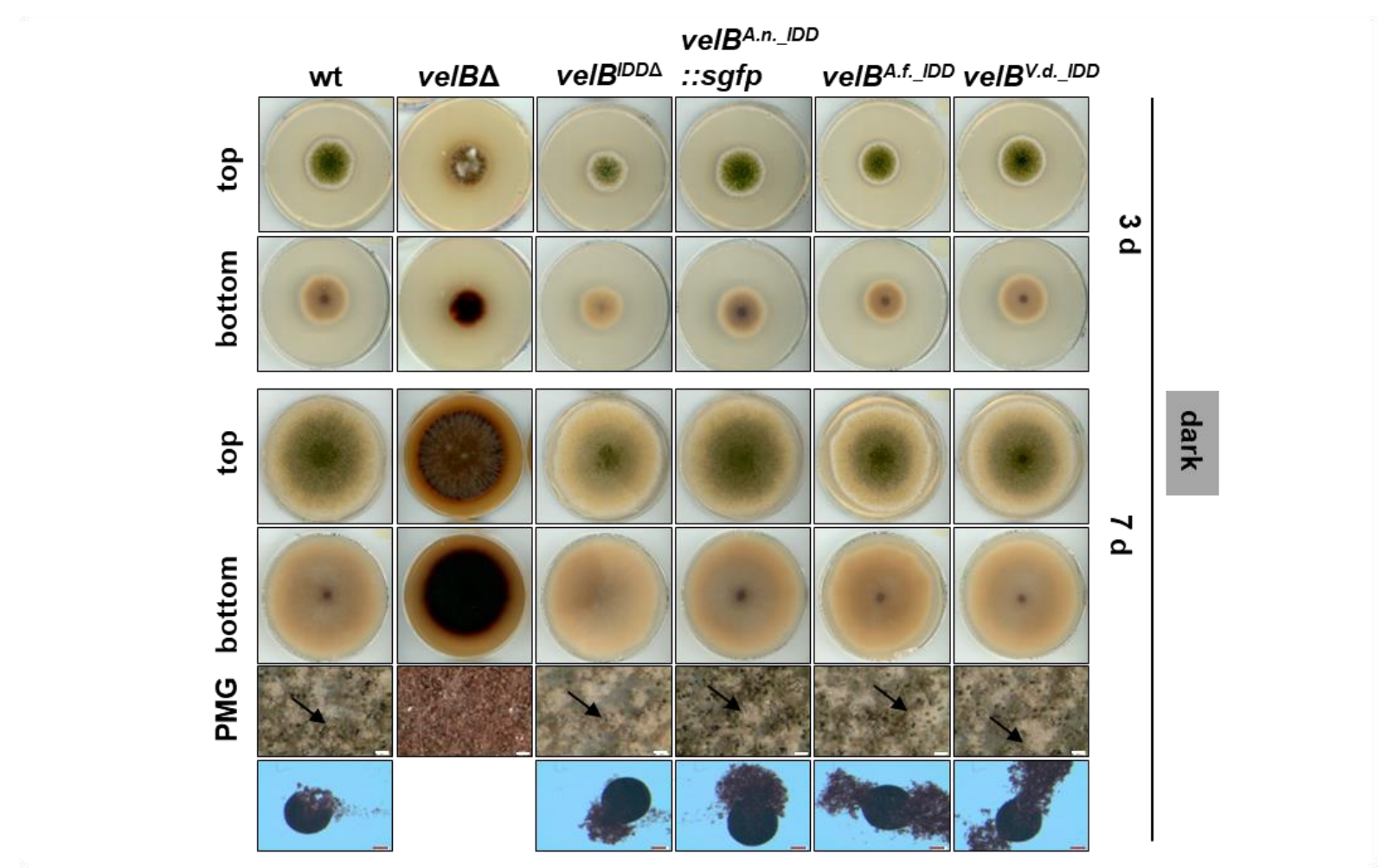

FIGURE 23: Cleistothecium formation and maturation is independent of the VelB IDD.

Phenotypes of wildtype (wt), velB deletion (velB $)$, velB $B^{I D D}$ deletion $\left(v e l B^{I D D \Delta}\right.$ ) and complementation with the IDD of $A$. nidulans, $A$. fumigatus or Verticillium dahliae into vel ${ }^{I D D \Delta}$

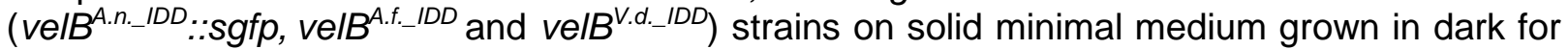
3 or 7 days $(\mathrm{d})$ at $37^{\circ} \mathrm{C}$. Black arrows indicate mature cleistothecia. $P M G=$ photomicrograph, scale bar $($ white $)=100 \mu \mathrm{m},($ red $)=50 \mu \mathrm{m}$. 
These findings indicate that the IDD is necessary for conidiation but dispensable for the formation of sexual fruiting bodies in $A$. nidulans. Taken together, these data support that the VelB-VeA heterodimer, which still can be formed without the VelB IDD, is necessary for sexual development. The VelB-VelB homodimer and the VelB-VosA heterodimer, which are not formed in the absence of the VelB IDD, are not needed for sexual development. Furthermore, the requisite VelB-VeA heterodimer ensures a fast light response, which results in the formation of conidiophores in hyphae growing from a single spot. In contrast to that, formation of the VelB-VeA heterodimer is not sufficient to form conidiophores from spores, which are surrounded by each other, due to a high spore density. The VelB-VosA and maybe also the VelB-VelB dimers are required in addition to the VeIB-VeA heterodimer to form conidiophores with air-borne conidiospores within the interconnected and branched mycelium at high spore densities.

\subsubsection{The survival of conidiospores is independent of the VelB IDD of} A. nidulans

The survival of conidiospores is decreased in the velB and $\operatorname{vos} A$ deletion strains and in both mutants the mRNA level of genes associated with the synthesis of trehalose is reduced (Ni and Yu, 2007; Park et al., 2012; Sarikaya-Bayram et al., 2010). This indicates that VelB and VosA are required for trehalose biosynthesis and the survival of spores. Lack of the VelB IDD abolishes heterodimer formation with VosA and therefore it was investigated whether this also influences conidiospore viability. The survival of conidiospores was examined in the $v e / B^{I D D_{\Delta}}$ mutant and compared to the spore viability of the wildtype, velB $\Delta$ and the $v e / B^{I D D_{\Delta}}$ strain complemented with velB::sgfp (velB $\left.B^{A . n .}{ }^{I D D}: .: s g f p\right)$. The same amount of spores was plated for all strains on solid minimal medium and after two, five and ten days spores were harvested and 200 spores plated again. After two days colony forming units were counted to draw a conclusion about the survival rate of conidia. Only $14 \%$ of the plated spores of the velB $\Delta$ strain survived and formed new colonies after ten days (FIGURE 24). In contrast, the survival rate of the $v e / B^{I D D_{\Delta}}$ showed similarities to the number of survived conidiospores of the wildtype. This indicates that the IDD is dispensable for conidiospore viability and that presumably not only the VelB-VosA heterodimer is required for the survival of conidiospores. 


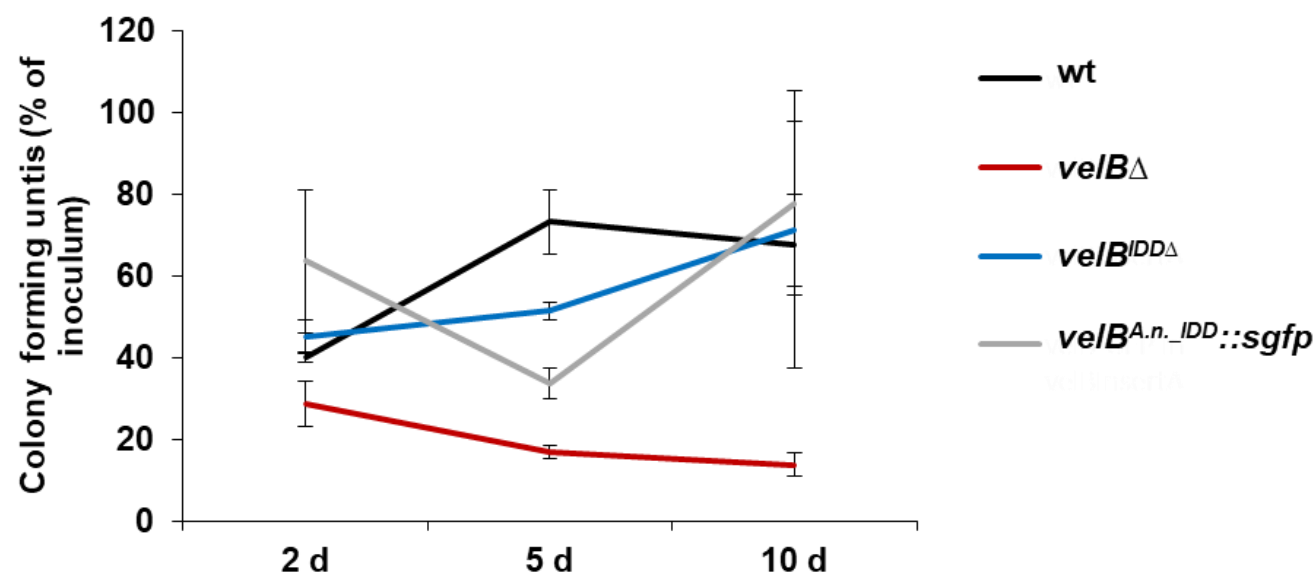

FIGURE 24: The VeIB IDD is dispensable for conidiospore viability.

Survival of conidiospores after 2, 5 or 10 days of wildtype (wt), velB deletion (velB $\Delta$ ), velB ${ }^{I D D}$ deletion $\left(v e / B^{I D D \Delta}\right)$ and vel$B^{I D D \Delta}$ complementation strain (velB $\left.B^{A . n ._{-} I D D_{-}}-G F P\right)$. For the velB deletion strain (velBA) just $14 \%$ of plated conidiospores survived after 10 days. Survival rate of wt, velB $B^{A . n .} I D D .:$ sgfp and velB ${ }^{I D D_{\Delta}}$ strains is between 50 and $100 \%$. Error bars indicate standard error of the mean (SEM) of three biological replicates.

\subsection{The VelB intrinsically disordered domain is required for the regulation of secondary metabolite production of $A$. nidulans}

\subsubsection{The accurate regulation of sterigmatocystin production requires the intrinsically disordered domain of VelB}

Secondary metabolism is interconnected with developmental programs in filamentous fungi and this interconnection is coordinated by velvet domain proteins. VelB-VeA forms the heterotrimeric velvet complex with the methyltransferase LaeA and regulates secondary metabolite genes at transcriptional or epigenetic level (Bayram et al., 2008; Sarikaya-Bayram et al., 2015; Strauss and Reyes-Dominguez, 2011). The production of the mycotoxin sterigmatocystin is under the control of the VelB-VeA-LaeA velvet complex with LaeA as the global regulator of secondary metabolism (Bayram et al., 2008). LaeA was also identified, but below threshold, in GFP pull downs of VelB with and without IDD. This indicates that the formation of the velvet complex is independent of the presence or absence of the intrinsically disordered domain of VelB. Deletion of $v e A$ or laeA abolishes sterigmatocystin production (Bayram et al., 2008; Kato et al., 2003). Lack of velB drastically reduces the production of sterigmatocystin (Bayram et al., 2008). Both veA $\Delta$ and velB $\Delta$ deletion mutants produce red brownish pigments released to the agar.

It was investigated whether the VelB-VosA or the VelB-VelB heterodimers, which are not formed in the absence of the VeIB IDD, are required for secondary metabolite production. Sterigmatocystin production of wildtype, velB deletion and $v e / B^{I D D_{\Delta}}$ mutant strains was compared and visualized by thin layer chromatography (TLC). 
After derivatization of the silica plates with $\mathrm{AlCl}_{3}$, detection at $366 \mathrm{~nm}$ revealed a drastically increased production of sterigmatocystin in the velB $B^{I D D_{\Delta}}$ mutant after three and seven days of growth in both, light and dark (FIGURE 25A). The production is increased to $500 \%$ in the velB $B^{I D D_{\Delta}}$ mutant, compared to wildtype (FIGURE 25B). This can be complemented by reintroducing the intrinsically disordered domain of $A$. nidulans and also by the IDD from $A$. fumigatus and $V$. dahliae. No sterigmatocystin could be detected in samples extracted from the velB $\Delta$ strain.

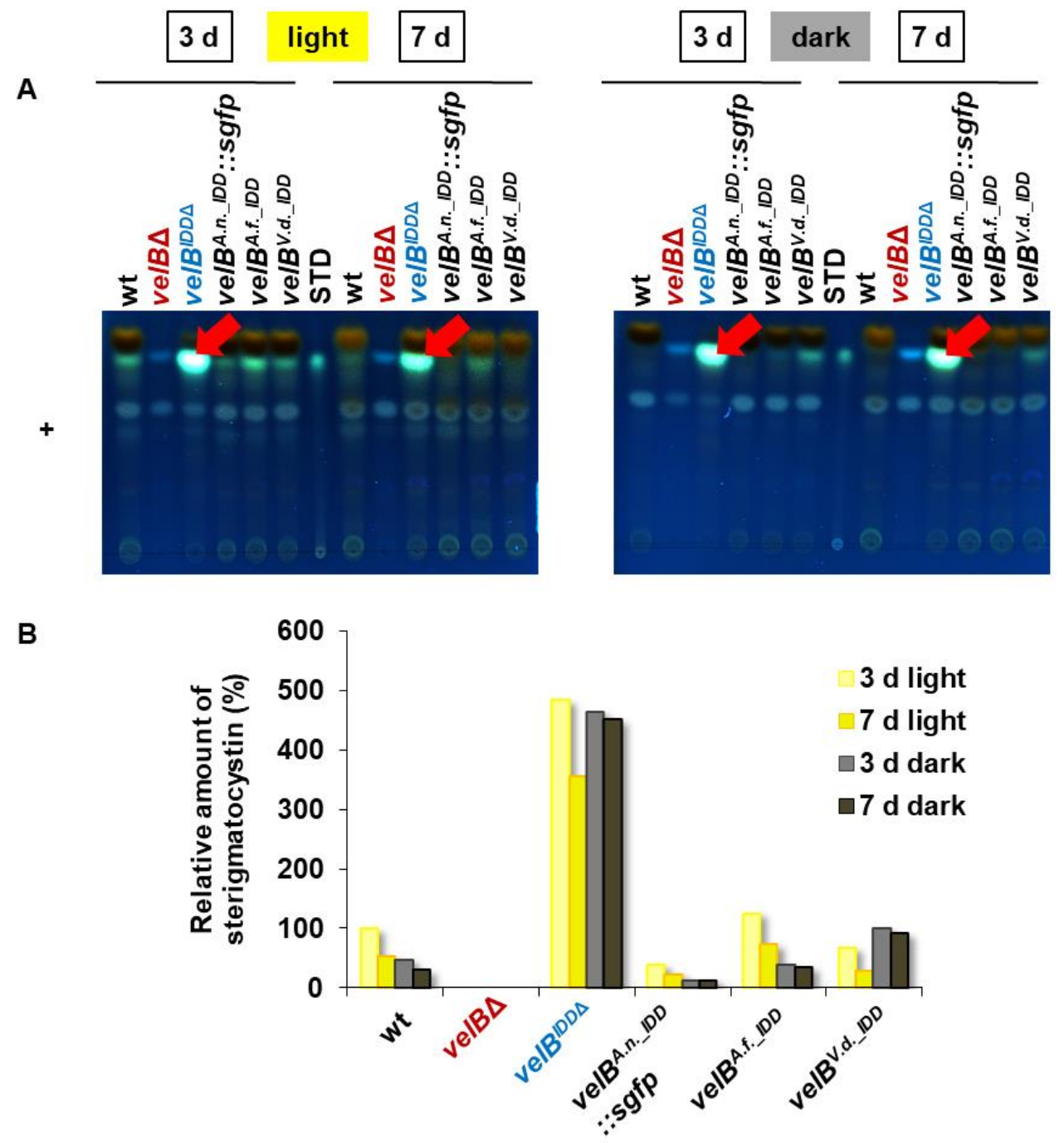

FIGURE 25: Accurate regulation of sterigmatocystin production requires the VelB IDD.

Sterigmatocystin production is increased in vel$B^{I D D} \Delta$ mutant (red arrow). Metabolite isolation of

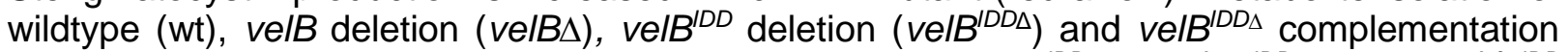

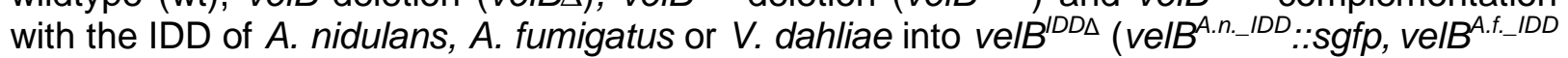

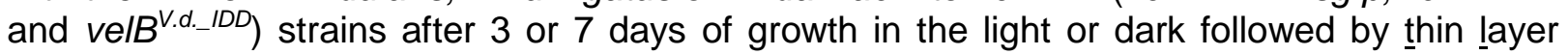
chromatography (TLC). The signals of the TLC plate were detected at $366 \mathrm{~nm}$ after (+) derivatization with $\mathrm{AICl}_{3}$. STD = Sterigmatocystin standard. B. Quantification of the sterigmatocystin signal relative to wt. 
The alteration of secondary metabolite production was further studied with high performance liquid chromatography (HPLC) and liquid chromatography coupled to mass spectrometry (LC-MS) experiments. A prominent peak (6) was detected in HPLC measurements of the velB $B^{I D D_{\Delta}}$ mutant during growth in light or dark (FIGURE 26).

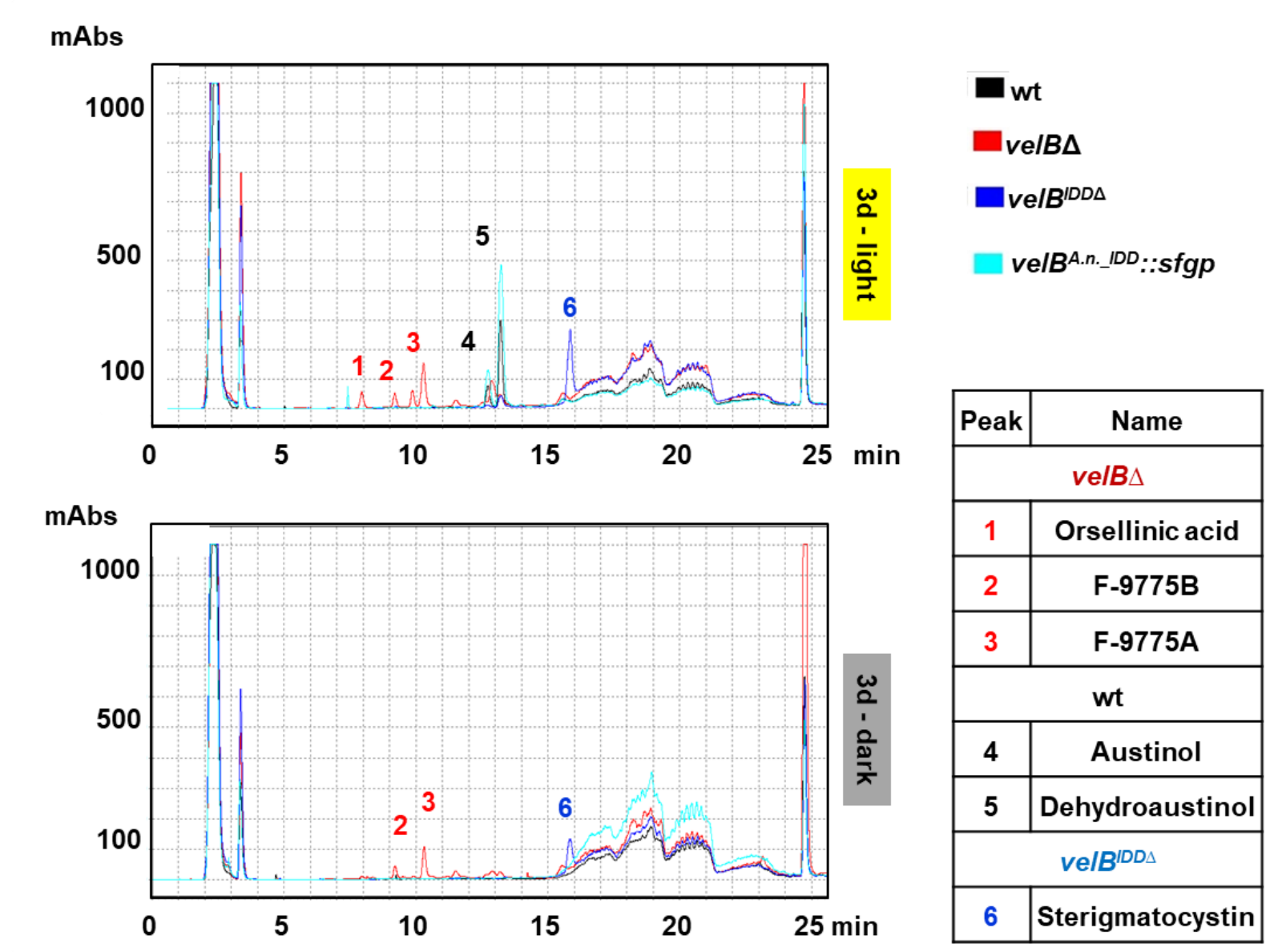

FIGURE 26: Secondary metabolite production is altered in the velB ${ }^{I D D \Delta}$ mutant.

High performance liquid chromatography was performed with metabolites extracted after 3 days of growth in the light or dark. Metabolites were isolated of wildtype (wt), velB deletion

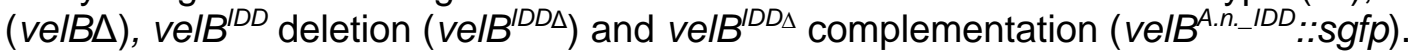

LC-MS analyses revealed a mass of $325.0714[\mathrm{M}+\mathrm{H}]^{+}$for this peak and due to the fragmentation of this mass (FIGURE 27B, TABLE 8), it was identified as sterigmatocystin (Szewczyk et al., 2008). This confirms the findings from the TLC. LC-MS analysis exhibited sterigmatocystin production also in the wildtype but with a 20 -fold lower intensity compared to the velB ${ }^{I D D_{\Delta}}$ mutant (FIGURE 27). Sterigmatocystin was not detected in the TLC experiments for the velB deletion strain (FIGURE 25) but LC-MS uncovered traces of sterigmatocystin with a tenfold decreased peak intensity compared to wildtype (FIGURE 27). Taken together, the VelB intrinsically disordered domain, which is required for the formation of the VelB-VosA and VelBVelB dimer but not for VelB-VeA heterodimer formation, is necessary for the accurate regulation of sterigmatocystin production. 


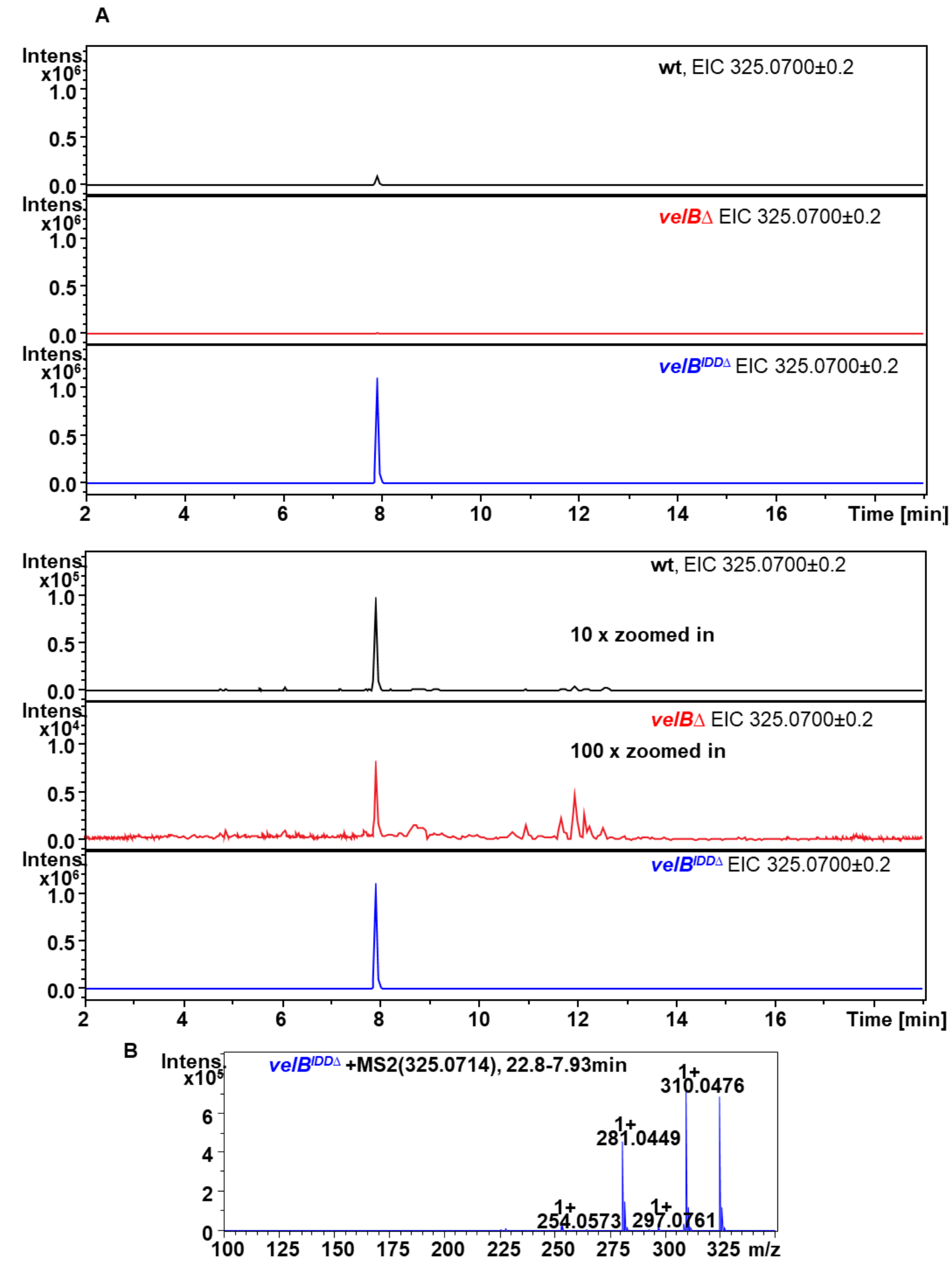

FIGURE 27: Sterigmatocystin production is decreased in the velB deletion mutant.

A. Liquid chromatography coupled to mass spectrometry revealed different sterigmatocystin amounts produced by wildtype (wt), velB deletion (velB $\Delta$ ) and the velB $B^{I D D_{\Delta}}$ mutant strain. $B . S^{2}$ spectrum of fragmentation of the sterigmatocystin peak with a mass of $325.0714[\mathrm{M}+\mathrm{H}]^{+}$. 


\subsubsection{The VelB-VosA heterodimer regulates austinol and dehydroaustinol biosynthesis}

VelB-VeA(-LaeA) is required for the activation of sterigmatocystin production, whereas the intrinsically disordered domain of VelB, and therefore VelB-VosA (and/or VelB-VelB), is necessary for accurate regulation of sterigmatocystin production. Sterigmatocystin is detectable under UV light of wavelength $366 \mathrm{~nm}$. UV light of wavelength $254 \mathrm{~nm}$ and white light were used for the visualization of other extracellular metabolites produced by the A. nidulans mutant strains. Detection of the silica plates at $254 \mathrm{~nm}$ and white light shows that production of other, so far unidentified, secondary metabolites is delayed (FIGURE 28, blue arrows). The white arrows indicate a secondary metabolite, which production is decreased in the velB ${ }^{I D D_{\Delta}}$ mutant in the light (FIGURE 28). HPLC and LC-MS analyses were conducted to get more insights into these alterations in secondary metabolite production.

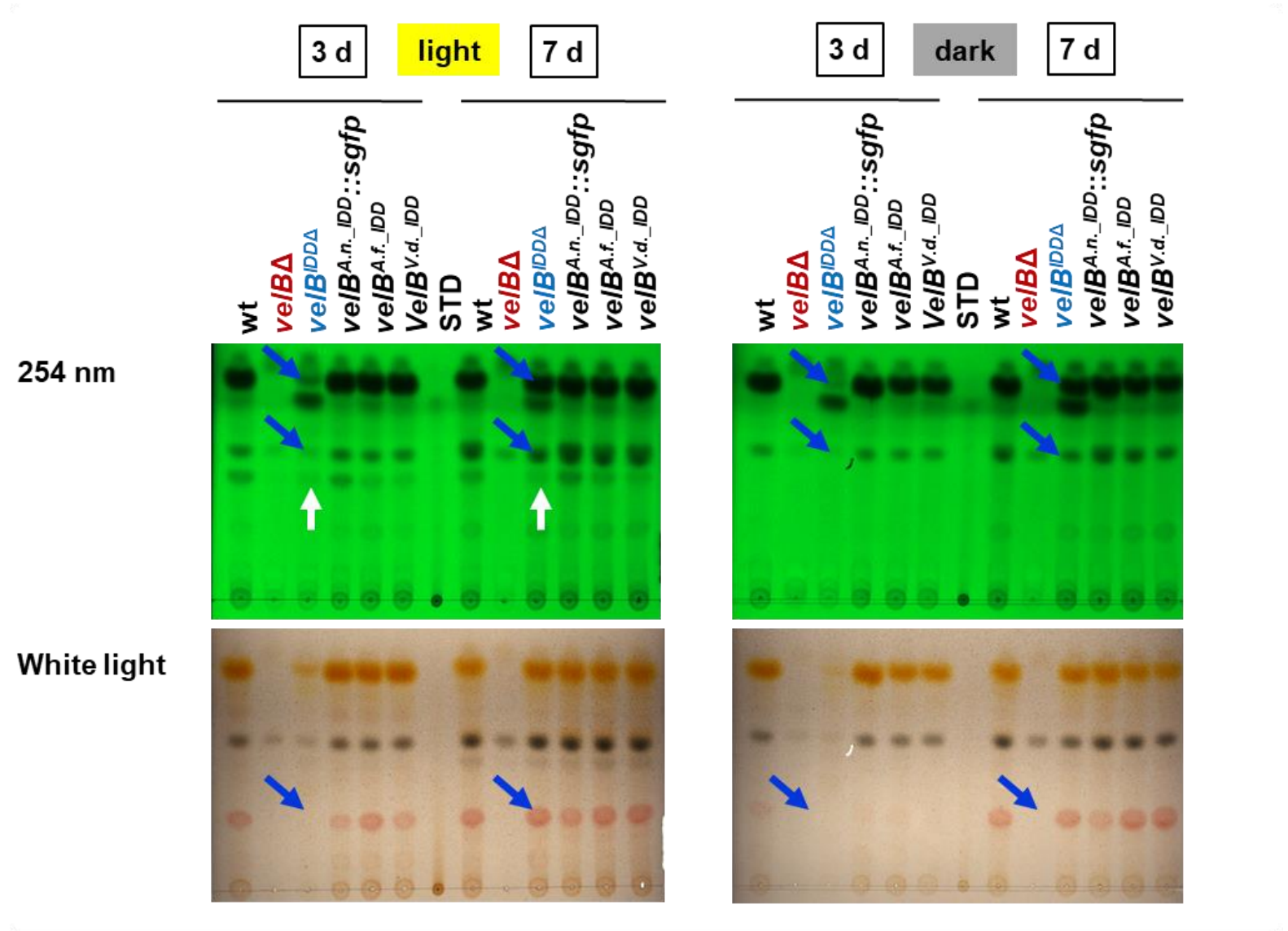

FIGURE 28: The VelB IDD promotes VelB-VosA heterodimer formation and regulates secondary metabolite production.

Thin layer chromatography of sterigmatocystin isolation detected at $254 \mathrm{~nm}$ and white light of

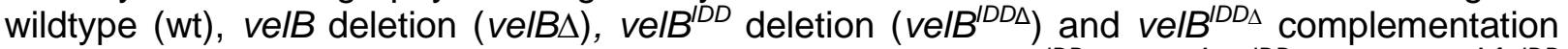

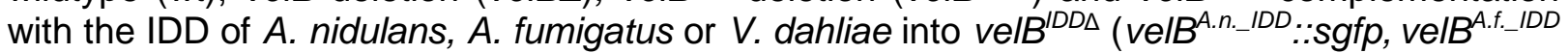

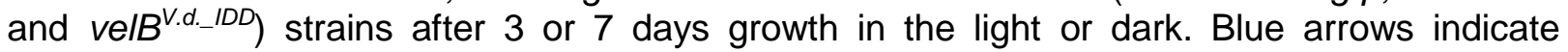
delayed production and white arrows decreased production of secondary metabolites in the velB ${ }^{I D D \Delta}$ mutant. 
Influence of changed velvet heterodimer formation on secondary metabolite production was examined in the wildtype, velB deletion, $v e / B^{I D D_{\Delta}}$ and the vel$/ B^{I D D_{\Delta}}$-complementation strain

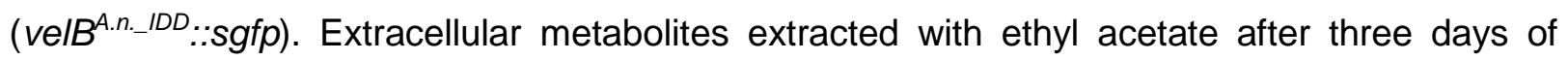
growth in light to induce asexual or darkness and limited oxygen supply to promote sexual development were compared. HPLC and LC-MS analyses revealed altered secondary metabolite production for the vel$B^{I D D_{\Delta}}$ strain, compared to wildtype.

Dehydroaustinol is one of the two compounds which were found to signal the induction of sporulation in A. nidulans (Gerke and Braus, 2014; Rodríguez-Urra et al., 2012). The wildtype and complementation strain both produce austinol (4) and dehydroaustinol (5). Production of these metabolites is drastically reduced in both, the velB deletion and the vel$B^{I D D_{\Delta}}$ mutant strain (FIGURE 26), which also produce less conidiospores (FIGURE 22). The velB deletion strain is completely abolished in the formation of heterodimers including VelB, whereas in the $v e / B^{I D D_{\Delta}}$ strain the interaction of VelB and VeA is still possible. Both strains are not capable of forming the VelB-VosA heterodimer. This suggests that the VelB-VosA heterodimer is a direct or indirect activator of austinol and dehydroaustinol production.

\subsubsection{The VelB-VeA heterodimer represses the production of orsellinic acid and F-9775A and $B$}

The velB deletion strain produces less austinol and dehydroaustinol and almost no sterigmatocystin compared to wildtype. This strain produces compounds, which were not identified for the wildtype or the velB ${ }^{I D D_{\Delta}}$ mutant (FIGURE 26).

It was shown that VeA acts as a repressor of the polyketide orsellinic acid gene cluster (Bok et al., 2013). The veA deletion mutant produces high amounts of orsellinic acid and its derivatives, the cathepsin $\mathrm{K}$ inhibitors $\mathrm{F}-9775 \mathrm{~A}$ and $\mathrm{B}$, which are also produced when A. nidulans is under stress conditions (Bok et al., 2013; Sanchez et al., 2010; Sarikaya-Bayram et al., 2015; Schroeckh et al., 2009). The veA $\Delta$ and the velB $\Delta$ mutants both produce a brown pigment, which is released into the agar. This suggests that they produce similar compounds, which are not present in the wildtype. Orsellinic acid and its derivatives F-9775A and B were identified in the velB deletion mutant, but nor in the vel $B^{I D D_{\Delta}}$ mutant neither in the wildtype, indicating that the VelB-VeA heterodimer represses the orsellinic gene cluster in wildtype situation (FIGURE 26, TABLE 8). 
TABLE 8: List of secondary metabolites identified via LC-MS analyses.

The table shows the secondary metabolites identified in the wildtype (wt), velB deletion (velBs), the $v e / B^{I D D}$ deletion mutant $\left(v e / B^{I D D_{\Delta}}\right)$ and the $v e / B^{I D D_{\Delta}}$ complementation strain (velB $\left.{ }^{A . n .} I D D .: s g f p\right)$. The peak numbers refer to the high performance liquid chromatogram from FIGURE 26. The mass-to-charge ratio $(\mathrm{m} / \mathrm{z})$ of the precursor ion, its fragmentation $\left(\mathrm{MS}^{2}\right)$ and the UV/Vis spectrum $\left(\lambda_{\max }\right)$ of each peak enable the identification (name) according to the literature (Bok et al., 2009; Frisvad and Thrane, 1987; Szewczyk et al., 2008).

\begin{tabular}{|c|c|c|c|c|c|c|}
\hline $\begin{array}{c}\text { Peak } \\
\text { number }\end{array}$ & Name & $\begin{array}{l}\text { Molecular } \\
\text { formula }\end{array}$ & $\mathrm{m} / \mathbf{z}$ & MS $^{2}$ & $\begin{array}{l}\lambda_{\max } \\
{[\mathrm{nm}]}\end{array}$ & References \\
\hline \multicolumn{7}{|c|}{ velB $\Delta$} \\
\hline 1 & Orsellinic acid & $\mathrm{C}_{8} \mathrm{H}_{8} \mathrm{O}_{4}$ & $\begin{array}{c}167.035 \\
{[\mathrm{M}-\mathrm{H}]^{-}}\end{array}$ & $\begin{array}{c}167.0349 \\
{[\mathrm{M}-\mathrm{H}]^{-}} \\
\\
147.0466 \\
\left.{ }^{-} \mathrm{M}-\mathrm{H}\right]^{-} \\
\\
123.0454 \\
{[\mathrm{M}-\mathrm{H}]^{-}} \\
\end{array}$ & $\begin{array}{l}215 \\
260 \\
300\end{array}$ & $\begin{array}{l}\text { (Frisvad and } \\
\text { Thrane, 1987) }\end{array}$ \\
\hline 2 & F-9775B & $\mathrm{C}_{21} \mathrm{H}_{16} \mathrm{O}_{8}$ & $\begin{array}{c}397.0925 \\
{[\mathrm{M}+\mathrm{H}]^{+}}\end{array}$ & $\begin{array}{c}323.0920 \\
{[\mathrm{M}+\mathrm{H}]^{+}}\end{array}$ & $\begin{array}{l}223 \\
305 \\
427\end{array}$ & $\begin{array}{c}\text { (Bok et al., } \\
\text { 2009) }\end{array}$ \\
\hline 4 & F-9775A & $\mathrm{C}_{21} \mathrm{H}_{16} \mathrm{O}_{8}$ & $\begin{array}{c}397.0923 \\
{[\mathrm{M}+\mathrm{H}]^{+}}\end{array}$ & $\begin{array}{c}287.0557 \\
{[\mathrm{M}+\mathrm{H}]^{+}} \\
151.0388 \\
{[\mathrm{M}+\mathrm{H}]^{+}}\end{array}$ & $\begin{array}{l}223 \\
305 \\
427\end{array}$ & $\begin{array}{l}\text { (Bok et al., } \\
\text { 2009) }\end{array}$ \\
\hline \multicolumn{7}{|c|}{ wildtype and velB ${ }^{A . n . I D D}:: s g f p$} \\
\hline 4 & Austinol & $\mathrm{C}_{25} \mathrm{H}_{29} \mathrm{O}_{8}$ & $\begin{array}{c}458.5009 \\
{[\mathrm{M}+\mathrm{H}]^{+}}\end{array}$ & l & $\begin{array}{l}210 \\
245\end{array}$ & $\begin{array}{c}\text { (Szewczyk et } \\
\text { al., 2008) }\end{array}$ \\
\hline 5 & Dehydroaustinol & $\mathrm{C}_{25} \mathrm{H}_{27} \mathrm{O}_{8}$ & $\begin{array}{c}456.4850 \\
{[\mathrm{M}+\mathrm{H}]^{+}}\end{array}$ & / & $\begin{array}{l}212 \\
240 \\
\text { (sh) }\end{array}$ & $\begin{array}{l}\text { (Szewczyk et } \\
\text { al., 2008) }\end{array}$ \\
\hline \multicolumn{7}{|c|}{ velB ${ }^{I D D \Delta}$} \\
\hline 6 & Sterigmatocystin & $\mathrm{C}_{18} \mathrm{H}_{11} \mathrm{O}_{6}$ & $\begin{array}{c}325.0714 \\
{[\mathrm{M}+\mathrm{H}]^{+}}\end{array}$ & $\begin{array}{c}310.0476 \\
{[\mathrm{M}+\mathrm{H}]^{+}} \\
297.0761 \\
{[\mathrm{M}+\mathrm{H}]^{+}} \\
281.0449 \\
{[\mathrm{M}+\mathrm{H}]^{+}} \\
254.0573 \\
{[\mathrm{M}+\mathrm{H}]^{+}}\end{array}$ & $\begin{array}{l}205 \\
245 \\
326\end{array}$ & $\begin{array}{l}\text { (Szewczyk et } \\
\text { al., 2008) }\end{array}$ \\
\hline
\end{tabular}


In summary, VelB can operate with the right interaction partner as positive or negative regulator for the production of certain secondary metabolites (TABLE 9). The VelB-VeA heterodimer activates together with LaeA as the heterotrimeric velvet complex sterigmatocystin production. The VelB-VeA heterodimer has also repressive functions. The VelB-VeA heterodimer is formed in wildtype situation and in the absence of the VeIB IDD and orsellinic acid and its derivatives F-97755A and B are not produced. Deletion of veA or velB results in the production of orsellinic acid and its derivatives. This indicates that the VelB-VeA heterodimer represses the biosynthesis of orsellinic acid and its derivatives, F-9775A and B. Austinol and dehydroaustinol are produced in the wildtype and complementation strain, whereas deletion of velB or only the IDD, which disables VelB-VosA heterodimer formation, results in decreased production of these secondary metabolites. This implies that VelB requires the interaction with VosA to function as activator for the production of austinol and dehydroaustinol.

TABLE 9: Specific dimer formation of velvet domain protein results in regulation of secondary metabolite gene clusters in $A$. nidulans.

The table presents possible dimer formation between VelB, VeA and VosA and secondary

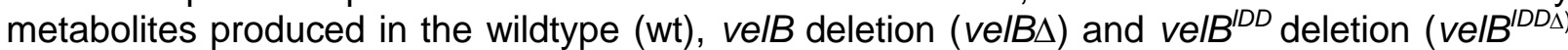
mutants. +: produced, +++: production increased, -: production decreased, /: not produced; $\checkmark=$ present,$x=$ absent.

\begin{tabular}{|c|c|c|c|c|c|c|}
\hline & $\begin{array}{l}\text { VelB- } \\
\text { VeA }\end{array}$ & $\begin{array}{l}\text { VelB- } \\
\text { VosA }\end{array}$ & $\begin{array}{l}\text { VelB- } \\
\text { VelB }\end{array}$ & Sterigmatocystin & $\begin{array}{l}\text { Orsellinic acid } \\
\text { \& F-9775A/B }\end{array}$ & $\begin{array}{l}\text { Austinol \& } \\
\text { dehydroaustinol }\end{array}$ \\
\hline wt & & $\nu$ & $\nu$ & + & l & + \\
\hline velB $\Delta$ & $x$ & $x$ & $x$ & - & + & - \\
\hline$v e / B^{I D D \Delta}$ & $\checkmark$ & $x$ & X & +++ & l & - \\
\hline
\end{tabular}




\subsection{The intrinsically disordered domain of VelB is a possible target for protein degradation}

\subsubsection{The VelB protein is destabilized by the IDD}

Deletion of the $v e l B^{I D D}$ influences heterodimer formation, decreases conidiospore formation and alters secondary metabolite production. VelB-VeA heterodimer formation is not abolished when the IDD is absent. It was investigated whether the VelB stability is altered in the absence of the IDD resulting in developmental and metabolic changes or if the stability of the VelB-VeA dimer itself is responsible. The expression level of velBwas compared to vel$B^{I D D_{\Delta}}$ expression in the respective strains to exclude a regulation at the transcriptional level. Strains were grown for 24 hours in submerged cultures, shifted to solid minimal medium plates and incubated under illumination, promoting asexual development. The relative normalized expression of $v e / B^{I D D_{\Delta}}$ compared to velB was not significantly altered from six to 18 hours of growth in the light (FIGURE 29).

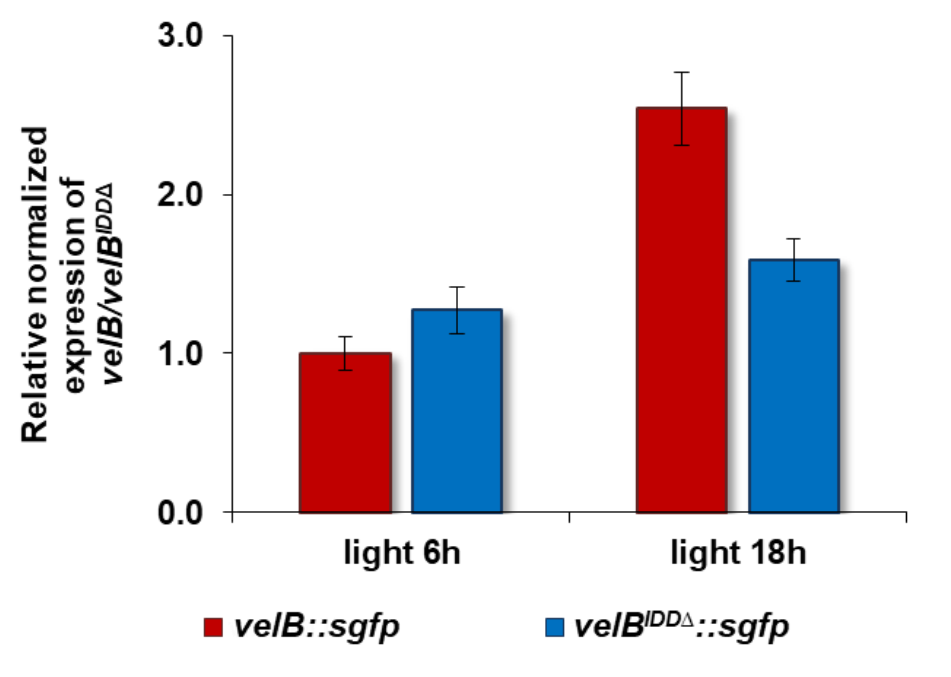

FIGURE 29: The relative expression of velB is not altered in a velB ${ }^{I D D} \Delta::$ sgfp expressing strain compared to a velB::sgfp expressing strain.

qRT-PCR analyses indicate that transcription of the truncated velB ${ }^{I D D_{\Delta}}::$ sgfp gene is not significantly regulated compared to the transcription of the full length velB::sgfp gene. Strains were grown in submerged cultures for 24 hours, shifted to solid minimal medium and incubated for 6 or 18 hours in the light at $37^{\circ} \mathrm{C}$. Data from two biological and three technical replicates are shown. Reference genes $15 S$ rRNA and h2A were used for normalization. 
The regulation of the abundance of a protein and the precise control of protein turnover is fundamental for cellular homeostasis. The half-life of proteins with intrinsically disordered domains is significantly shorter than of structured proteins without unordered segments (Latysheva et al., 2015; van der Lee et al., 2014). The protein stability of the full length VelB-GFP compared to the VelB-GFP lacking the IDD was analyzed.

In a first approach, the relative protein amount during different developmental stages was investigated. Whole cell protein extracts were analyzed by SDS PAGE followed by western hybridization. The VelB-GFP protein with a molecular mass of $67 \mathrm{kDa}$ and the VelB ${ }^{\mathrm{IDD}} \Delta_{-}$GFP protein with a molecular mass of $56 \mathrm{kDa}$ are detectable in samples from cultures grown vegetatively for 24 hours (FIGURE 30A, veg 24h). The immunoblot revealed that VelB-GFP was detectable after three and six hours in the light, promoting asexual development as well as in the dark, inducing sexual development (FIGURE 30A). The relative VelB-GFP protein amount decreases during continuing development. Stable VelB-GFP protein was barely observed after 12 hours during ongoing sexual or asexual development and several degradation products become visible. After 12 hours of growth either in the light or dark a signal corresponding to the size of free GFP ( $27 \mathrm{kDa}$ ) could be observed (FIGURE 30A, orange arrow). This indicates that the protein is unstable and degraded rapidly during ongoing fungal development. The $V{ } B^{I D D}{ }^{I}$-GFP fusion protein amount also decreases during development but in contrast to the full length protein, $30 \%$ of the relative protein amount is still detectable in samples grown for 48 hours in light or dark compared to protein amounts from vegetatively grown cultures (FIGURE 30B). Free GFP, indicating degradation of parts of the total cellular VelB ${ }^{\text {IDD }}{ }_{-}$GFP protein amount, becomes visible after 24 hours of growth in the light or after 18 hours of growth in the dark (FIGURE 30A). Quantification of the relative protein amount confirmed that lack of the VeIB IDD results in increased protein abundance during developmental stages, compared to VelB-GFP harboring the intrinsically disordered domain (FIGURE 30B). This indicates that absence of the VeIB IDD alters the protein stability in $A$. nidulans. 


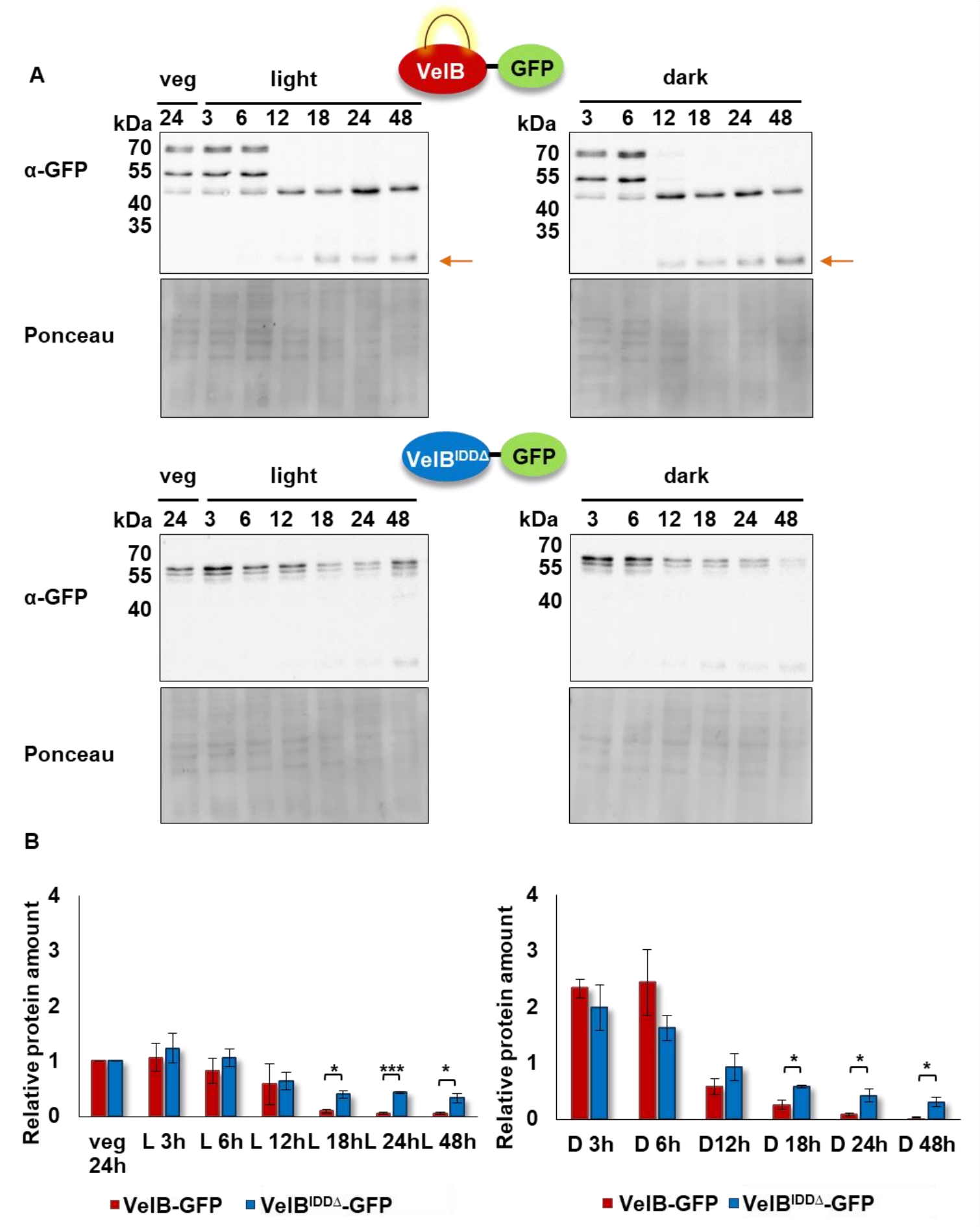

FIGURE 30: The IDD destabilizes VelB-GFP during fungal development.

The same amount of spores was inoculated in submerged cultures. For asexual/sexual development, the vegetative grown mycelia were shifted after $24 \mathrm{~h}$ to solid minimal medium and grown for indicated time spans (in hours) in light or dark at $37^{\circ} \mathrm{C}$. VelB-GFP is degraded over time and degradation products become visible, whereas stable VelB ${ }^{\operatorname{IDD} \Delta}$-GFP could still be detected after $48 \mathrm{~h}$ in light and dark. Orange arrow = free GFP. B. Quantification of VelB-GFP and $\mathrm{VelB}^{\mathrm{IDD}} \Delta_{-}$GFP relative to the protein amount of vegetative grown cultures normalized against Ponceau. $L=$ light, $D=$ dark. Error bars indicate standard error of the mean (SEM) of three biological replicates. $p$-value was calculated with standard deviation. ${ }^{* *}, p<0.01 ;{ }^{* \star *}, p<$ 0.005 
Further, cycloheximide assays were conducted to exclude translational effects upon protein degradation. Cycloheximide is a translational inhibitor, interfering with the translocation step in mRNA translation, which results in a block of translational elongation (Buchanan et al., 2016). Mycelia were treated with cycloheximide after 24 hours of vegetative growth to inhibit protein synthesis. The decrease in relative protein amounts over time was investigated (FIGURE 31). Differences of relative protein amounts of $25 \%$ between VelB with or without the intrinsically disordered domain were observed after one hour. The relative amount of VelB-GFP decreased to $50 \%$ after five hours. In contrast, $80 \%$ of the VelB ${ }^{\mathrm{IDD}}{ }_{\text {-GFP }}$ fusion protein compared to untreated mycelium was detected.

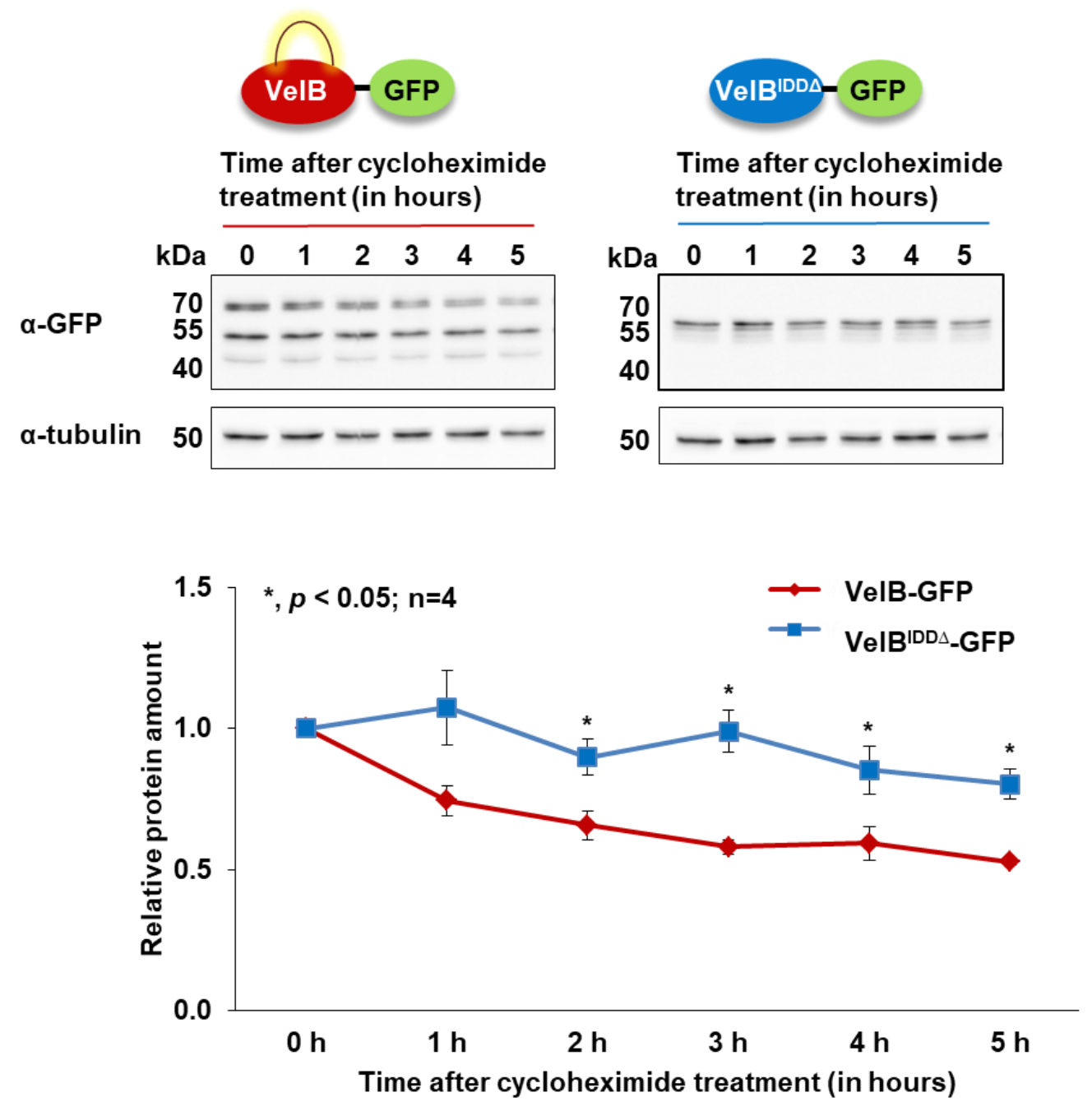

FIGURE 31: The IDD destabilizes VelB-GFP.

Translational inhibition with cycloheximide reveals that lack of the IDD of VelB increases its protein stability. The same amount of spores was inoculated in submerged cultures. $2.5 \mathrm{mg}$ cycloheximide was added after 24 hours (h) of growth at $37^{\circ} \mathrm{C}$ and samples were taken hourly. Error bars indicate standard error of the mean (SEM) of four biological replicates normalized against the tubulin signal. $p$-value was calculated with standard deviation. ${ }^{*}, p<0.05$. 
These data demonstrate that the stability of a VelB with or without the intrinsically disordered domain is different. Conclusively, VelB-GFP is destabilized by the intrinsically disordered domain and the absence of the IDD increases the stability of the VelB protein. It is likely that, under the right conditions, the cell controls the protein turnover of VelB by using the IDD as putative target for protein degradation.

\subsubsection{Fbx18 reduces cellular VelB protein levels comprising the intrinsically disordered domain}

The VelB IDD destabilizes the protein and in absence of the IDD protein degradation occurred not as fast as for the full length VelB protein. Key of the half-life control of proteins is the degradation mechanism determined by the ubiquitin-dependent proteasomal pathway. E3 ubiquitin ligases recruit substrates for degradation by the proteasome (Ciechanover, 1998; Jöhnk et al., 2016). F-box proteins determine the substrate specificity within E3-ligases (von Zeska Kress et al., 2012).

74 fbox encoding genes were identified in the genome of $A$. nidulans, which harbor the Fbox domain, consisting of around 45 amino acids (Ö. Bayram, p.c.). The deletion library of 72 fbox encoding genes was available. The respective fbox gene was exchanged through homologous recombination against the nutritional pyrG marker gene, which encodes an orotidine-5'phosphate decarboxylase. The background strain harbors a mutation in this gene (pyr89), rendering the fungus auxotroph for uridine and uracil. Successful transformation was monitored by the complementation of the auxotrophy that is caused by the pyrG89 mutation (Ö. Bayram, p.c.). It was investigated whether the VelB protein level is reduced by F-box proteins in $A$. nidulans and the relative protein amount of VelB was examined in 72 fbox deletion strains during vegetative growth (FIGURE 32) (fbox25 is essential for fungal growth and the fbox50 deletion mutant could not be resurrected from the strain collection). 


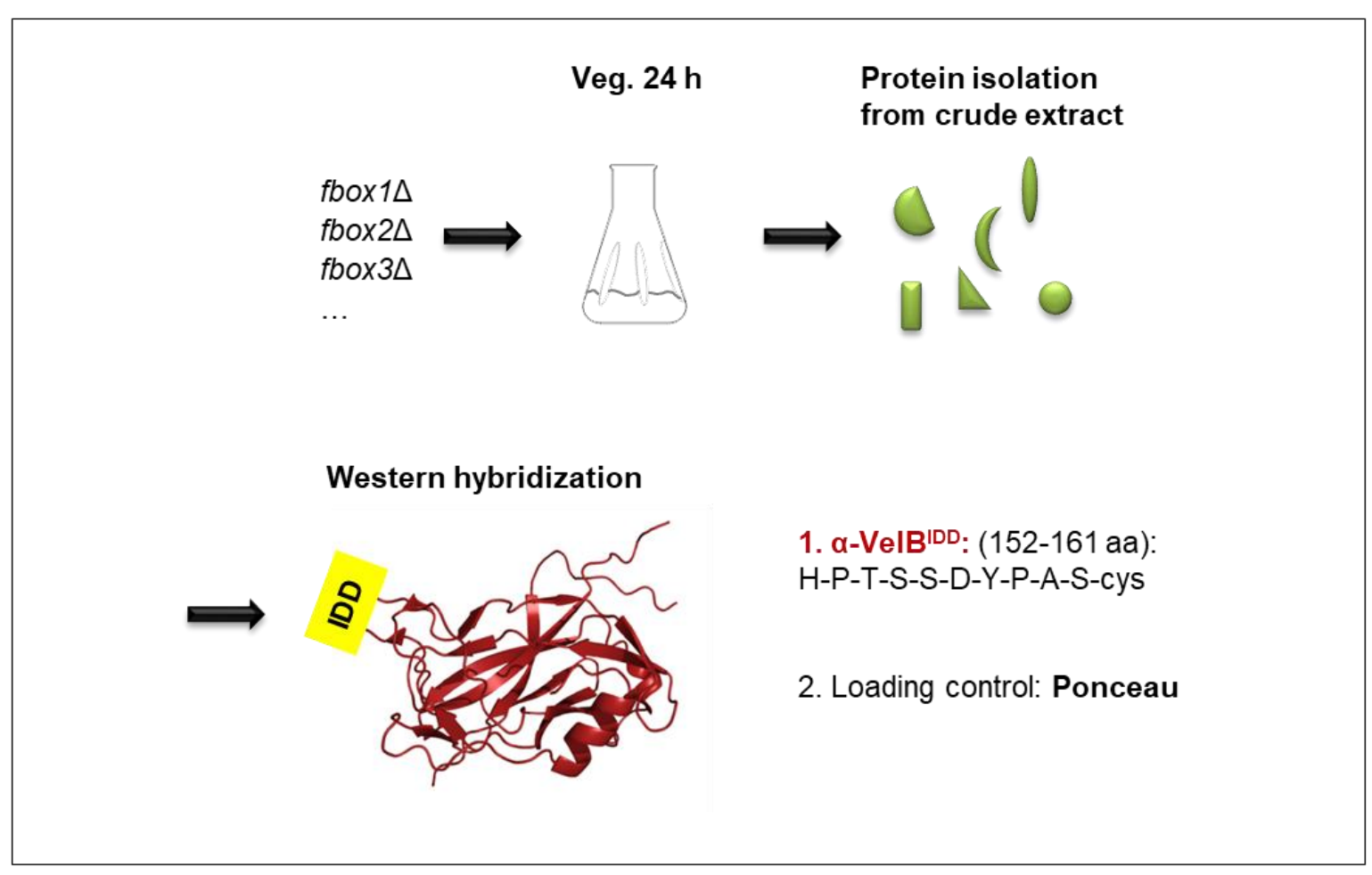

FIGURE 32: Investigation of the relative amount of VelB in fbox deletion mutants in A. nidulans.

This workflow depicts the procedure which was conducted to pre-screen the fbox deletion mutants for the relative VelB protein amount. Strains were grown vegetatively (veg.) in submerged cultures for 24 hours $(h)$ followed by protein isolation from crude extracts. Application of the monoclonal antibody against a sequence of the IDD of VelB revealed relative protein amounts and staining with Ponceau served as loading control.

The relative protein amount was detected with a monoclonal antibody against a sequence of the intrinsically disordered domain of VelB ( $\left.\alpha-\left.V_{e}\right|^{I D D}\right)$ and staining of the membrane with Ponceau served as loading control (FIGURE 33). Promising candidates identified in this pre-screen - highlighted in red (decreased protein amount of VelB) and green (increased VelB protein amount) - were further investigated (FIGURE 34). 


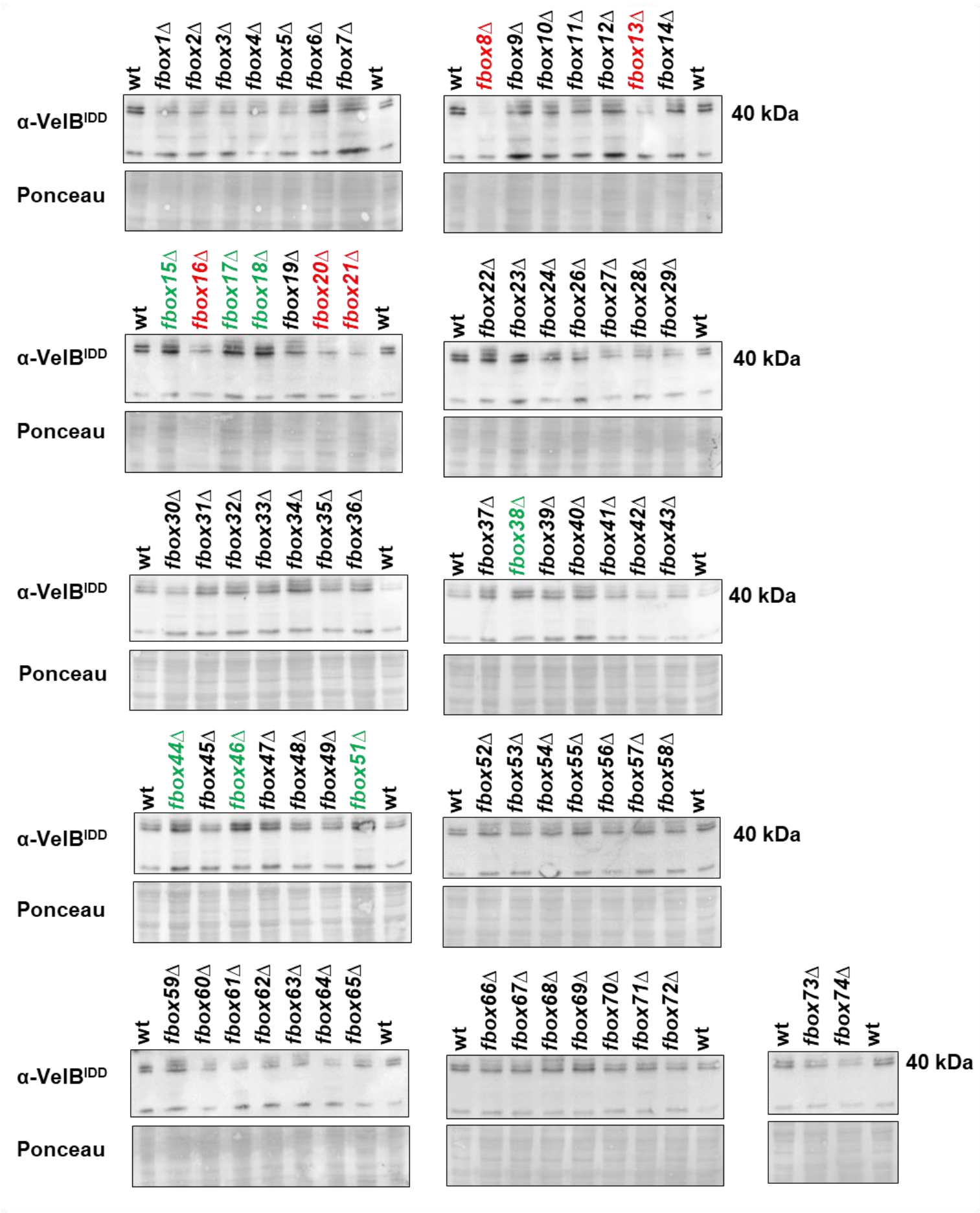

FIGURE 33: Examination of the relative amount of VelB in 72 fbox deletion strains of A. nidulans.

Relative protein amount was visualized with an antibody against a sequence of the VeIB IDD $\left(\alpha-V e B^{I D D}\right)$ and Ponceau staining was used as loading control. Crude extracts were prepared from 24 hours vegetatively grown cultures. fbox deletion strains were screened for alteration of VelB protein amount. Color codes refer to promising candidates with increased (green) or decreased (red) VelB protein amount. These candidates were chosen for further investigations. 
The mutants highlighted in green or red in FIGURE 33 where further investigated and the relative VelB protein amount was quantified. The signal detected for the $\alpha$-actin antibody served as loading control. The examination of these candidates revealed that the VelB protein level was significantly increased about two-fold in the fbox18 deletion mutant (FIGURE 34). The relative amount of VelB was not significantly altered in the fbox8, fbox13, fbox16, fbox17, fbox20, fbox21, fbox38, fbox44, fbox46, and fbox51 deletion mutants (FIGURE 34). Here, the fbox18 deletion mutant was further investigated regarding the relative amount of VelB.

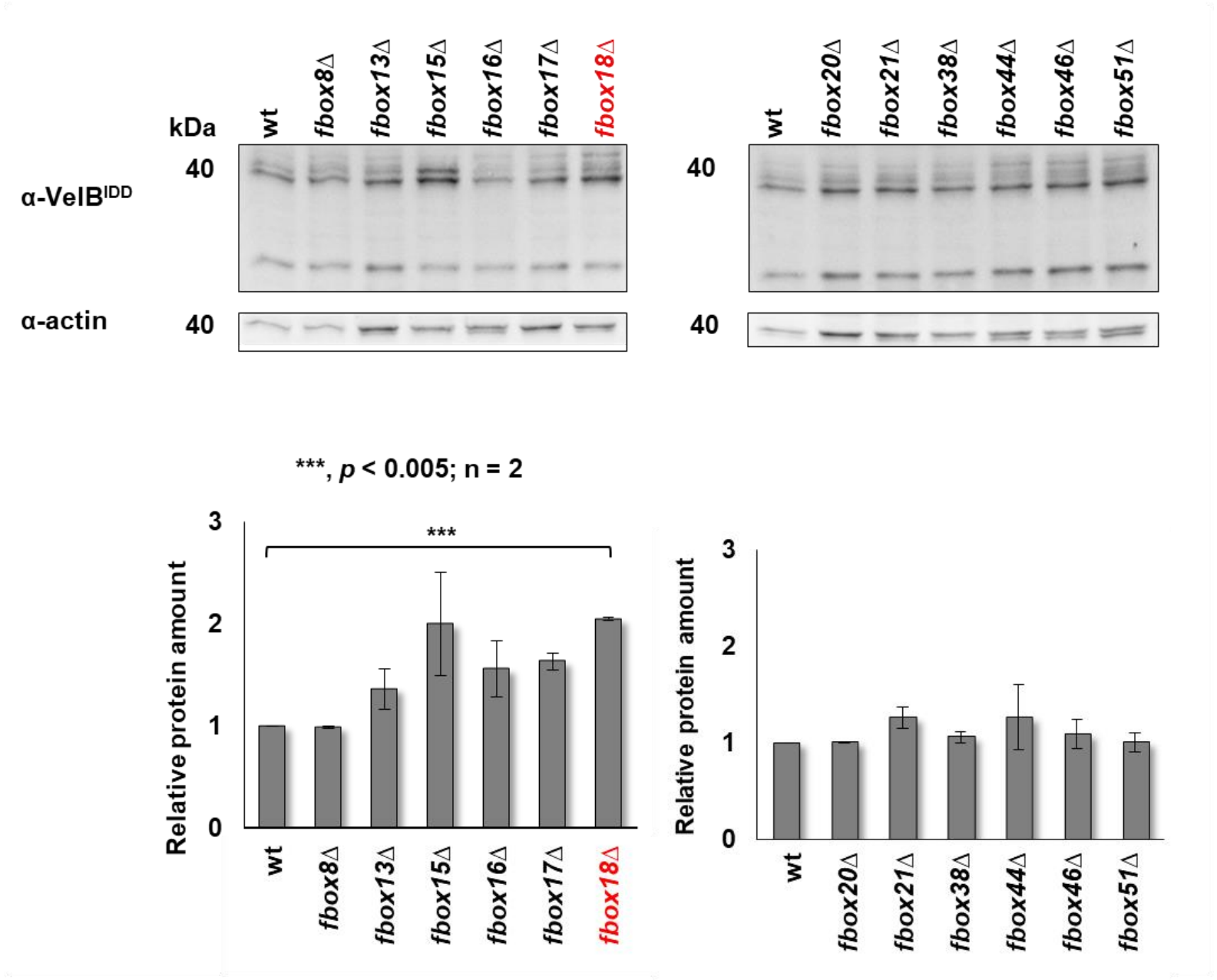

FIGURE 34: VelB protein level is significantly increased in the fbox18 $\Delta$ mutant.

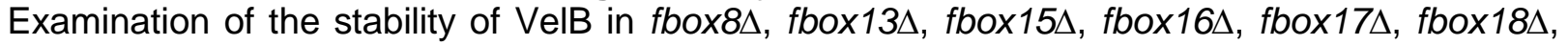

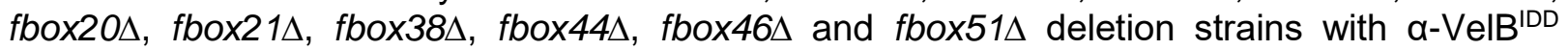
antibody and quantification of relative protein amount of VelB. Protein amount was normalized to the actin signal. Error bars indicate standard error of the mean (SEM) of two biological replicates. $p$-value was calculated with standard deviation. ${ }^{* * *}, p<0.005$. 
The protein stability of VelB-GFP in the fbox 18 deletion mutant was compared to the stability in the wildtype background. Cycloheximide assays were utilized to exclude translational effects on cellular protein levels. The experiment revealed that VelB-GFP is more stable in the absence of fbox 18 compared to wildtype situation (FIGURE 35). This was observed over a time course of five hours. The relative VelB-GFP amount remains stable (around 90\%) in the fbox 18 deletion mutant during the investigated time span, while it decreased to $75 \%$ in wildtype after one hour and continued to decrease to $50 \%$ in wildtype after five hours.
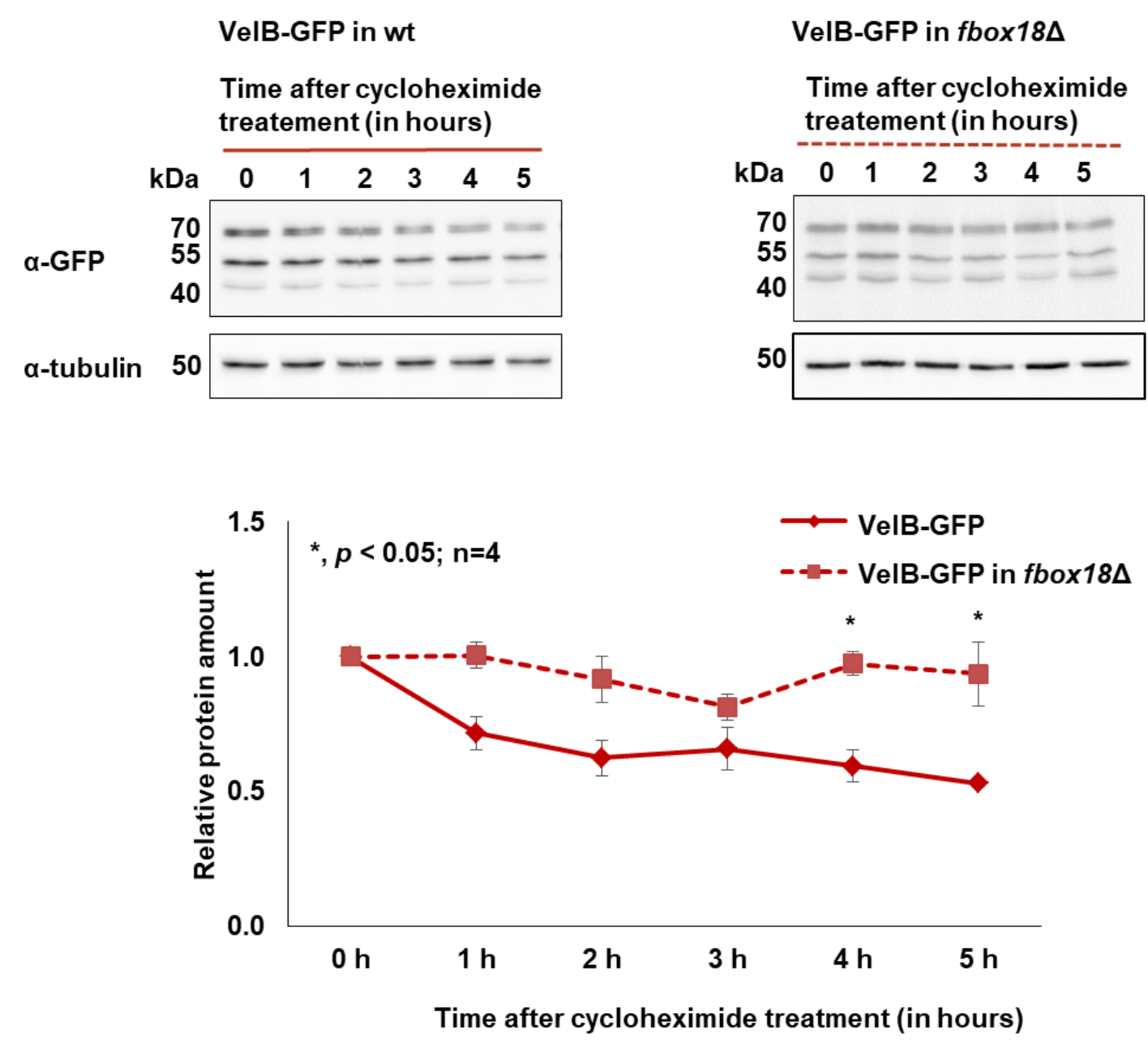

FIGURE 35: The stability of VelB is dependent on fbox18 in A. nidulans.

Translational inhibition with cycloheximide reveals that VelB stability is increased in the fbox 18 deletion mutant (fbox18s) compared to wildtype (wt). The same amount of spores was inoculated in submerged cultures. $2.5 \mathrm{mg}$ cycloheximide was added after 24 hours (h) of growth at $37^{\circ} \mathrm{C}$ and for 5 hours (h) samples were taken hourly. Error bars indicate standard error of the mean (SEM) of four biological replicates normalized against the tubulin signal. $p$-value was calculated with standard deviation. ${ }^{*}, p<0.05$. 
Deletion of $f b \times 18$ increases the relative protein amount of VelB but phenotypical analysis revealed no significant differences of the $f b \times 18 \Delta$ mutant strain compared to wildtype at first glance (FIGURE 36). This might indicate that increased amounts of full length VelB do not significantly affect asexual development in $A$. nidulans or that other F-box proteins serve as substrate-adaptor for VelB under these conditions.

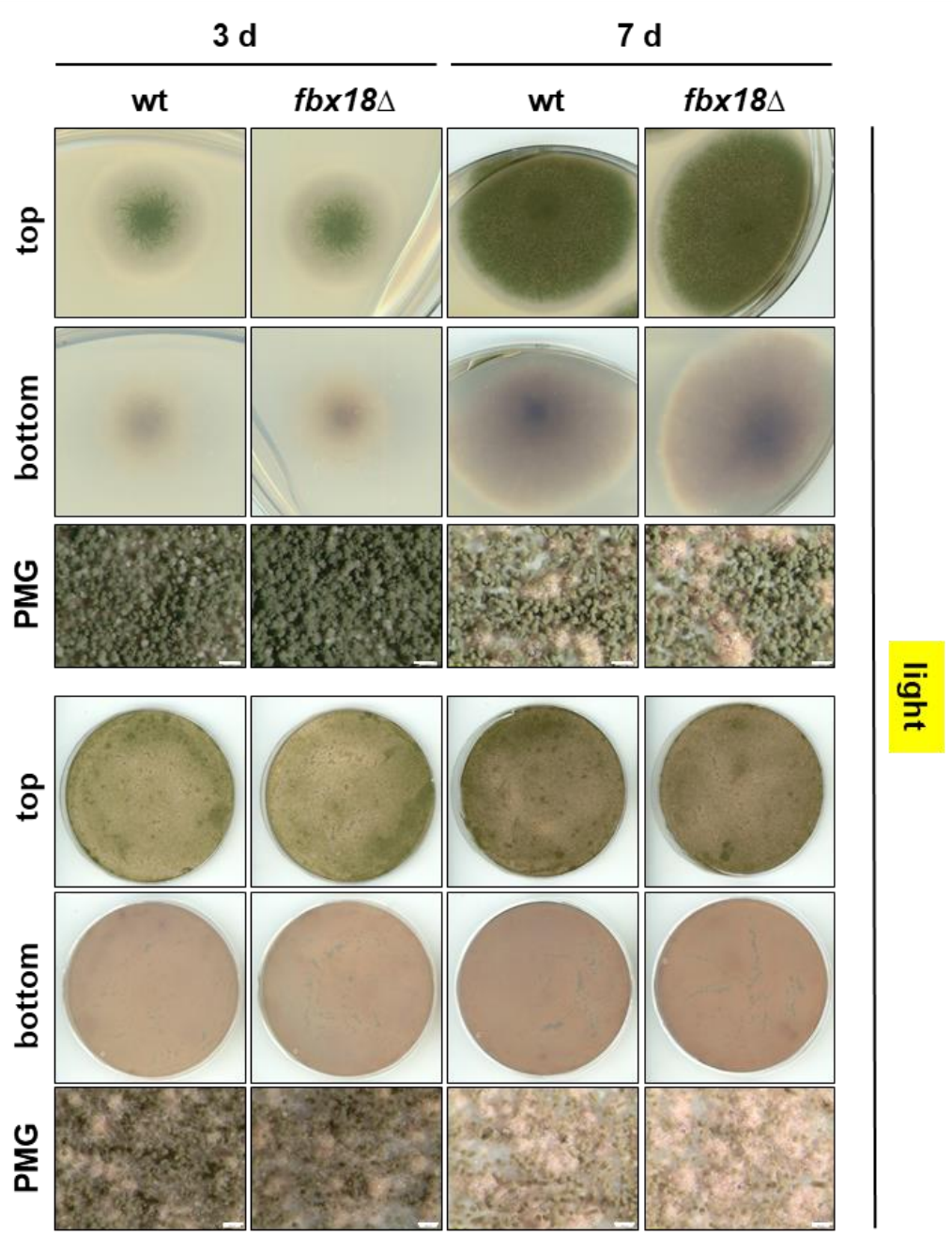

FIGURE 36: Phenotypical analysis of $f b \times 18 \Delta$ compared to wildtype.

Phenotypes of wildtype (wt) and fbx18 deletion ( $f b x 18 \Delta)$ strains on solid minimal medium. Either 2000 spores were point inoculated or $10^{5}$ spores were distributed over the whole plate and incubated for 3 or 7 days (d) at $37^{\circ} \mathrm{C}$ in the light. Scale bar $=100 \mu \mathrm{m}$, PMG $=$ photomicrograph.

Taken together, these data suggest that the VeIB IDD might be a substrate for the cellular protein degradation machinery, indicating Fbox18 as required factor, which reduces cellular VelB protein levels during vegetative growth conditions. Other F-box proteins might be responsible during other developmental programs to promote VelB degradation. 
3.6 In silico analyses of the A. nidulans VelB intrinsically disordered domain

The VeIB IDD destabilized the protein and might be a target for substrate-adaptors, such as Fbx18, which might label VelB for protein degradation. LC-MS/MS analysis of the VelB-GFP fusion protein did not detect peptides of the VeIB IDD and putative PTMs of the VelB intrinsically disordered domain could not be identified. Trypsin cleaves polypeptides principally at the C-terminal site of lysine or arginine. The VeIB IDD does not contain any of these residues and is therefore not cleaved into (for the LC-MS/MS) detectable peptide. Therefore, the sequence was examined in more detail in silico.

\subsubsection{The VelB intrinsically disordered domain of $A$. nidulans contains putative Short Linear Motifs}

The presence of Short Linear peptide Motifs (SLiMs), usually situated in intrinsically disordered regions, might be a potential regulator for VeIB IDD function, regarding dimerization or protein stability. They enable a heterogeneity in the protein interaction surface or possess residues for posttranslational modifications, which alter the chemical nature of the polypeptide and lead to an alteration of interaction partners (Babu et al., 2012; Buljan et al., 2013; Davey et al., 2012; Gsponer and Babu, 2009; Mao et al., 2013). SLiMs are very short and consist of only three to ten amino acids, whereas just two to four residues are strictly required for interaction with the motif-recognizing protein (Gouw et al., 2017; Weatheritt et al., 2012). Therefore, the binding of the interaction partner to these short motifs occurs with low affinity, which renders them difficult to be detected experimentally (Weatheritt et al., 2012).

In the present study, the Eukaryotic Linear Motif (ELM) (Dinkel et al., 2016) search was employed to investigate possible SLiMs in the VelB intrinsically disordered domain of A. nidulans. Several motifs were predicted for the complete sequence of VelB but SLiMs are typically only functional in intrinsically disordered regions and likely not inside structured domains (Gouw et al., 2017). The 14 linear motifs identified in the analysis within the 99 amino acid VeIB IDD sequence are listed in TABLE 10. 
TABLE 10: Short linear motifs of the VelB intrinsically domain of $\boldsymbol{A}$. nidulans.

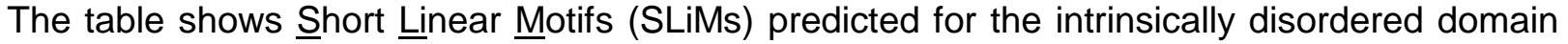
of VelB employing the bioinformatics resource ELM (Dinkel et al., 2016). A low score indicates a higher probability for the motif to be true and probability scores lower than 0.01 are highlighted in red.

\begin{tabular}{|c|c|c|c|c|}
\hline $\begin{array}{l}\text { ELM } \\
\text { name }\end{array}$ & Sequence & ELM Description & Pattern & Score \\
\hline $\begin{array}{l}\text { DEG } \\
\text { SPOP } \\
\text { SBC_1 }\end{array}$ & AMSSS & $\begin{array}{l}\text { S/T rich motif known as the SPOP-binding } \\
\text { consensus (SBC) of the MATH-BTB protein } \\
\text { is present in substrates that undergo } \\
\text { SPOP/Cul3-dependent ubiquitination. }\end{array}$ & $\begin{array}{l}{[\mathrm{AVP}] .[\mathrm{ST}][\mathrm{ST}][} \\
\mathrm{ST}]\end{array}$ & 0.000938 \\
\hline $\begin{array}{l}\text { DOC_- } \\
\text { CKS1_1 }\end{array}$ & PVTPGP & $\begin{array}{l}\text { Phospho-dependent motif that mediates } \\
\text { docking of CDK substrates and regulators } \\
\text { to cyclin-CDK-bound Cks1 }\end{array}$ & $\begin{array}{l}\text { [MPVLIFWYQ]. } \\
\text { (T)P.. }\end{array}$ & 0.001991 \\
\hline $\begin{array}{l}\text { DOC } \\
\text { USP7_-_ } \\
\text { MATH_1 }\end{array}$ & $\begin{array}{l}\text { AMSSS } \\
\text { PTSSD }\end{array}$ & $\begin{array}{l}\text { The USP7 MATH domain binding motif } \\
\text { variant; USP7 is responsible for the } \\
\text { deubiquitination of many cellular proteins }\end{array}$ & $\begin{array}{l}{[P A]\left[^{\wedge} P\right]\left[^{\wedge} F Y W I\right.} \\
L] S\left[{ }^{\wedge} P\right]\end{array}$ & 0.01239 \\
\hline $\begin{array}{l}\text { DOC_ } \\
\text { WW_- } \\
\text { Pin1_4 }\end{array}$ & $\begin{array}{l}\text { SATSPS } \\
\text { QPVTPG }\end{array}$ & $\begin{array}{l}\text { The Class IV WW domain interaction motif } \\
\text { is recognized primarily by the Pin } 1 \\
\text { phosphorylation-dependent prolyl } \\
\text { isomerase. }\end{array}$ & $\ldots([S T]) P$ & 0.01543 \\
\hline $\begin{array}{l}\text { LIG_ } \\
\text { FHA_2 }\end{array}$ & HPTSSDY & Phospho-threonine motif binding & $. .(T) . .[D E]$. & 0.008286 \\
\hline $\begin{array}{l}\text { LIG } \\
\text { WD40_- } \\
\text { WDR4_- } \\
\text { VDV_2 }\end{array}$ & $\begin{array}{l}\text { SATSPSI } \\
\text { TSPSI } \\
\text { SPSI }\end{array}$ & $\begin{array}{l}\text { Fungi-specific variant of the WDR5-binding } \\
\text { motif; which mediates assembly of histone } \\
\text { modification complexes. }\end{array}$ & $\begin{array}{l}\text { [EDSTY].\{0,4\}[ } \\
\text { VIPLA][TSDEK } \\
\text { R][ILVA] }\end{array}$ & 0.04678 \\
\hline $\begin{array}{l}\text { MOD_} \\
\text { CK1_1 }\end{array}$ & $\begin{array}{l}\text { SATSPSI } \\
\text { SISTAMS } \\
\text { SYQTNPY }\end{array}$ & CK1 phosphorylation site & S..([ST])... & 0.01704 \\
\hline $\begin{array}{l}\text { MOD__ } \\
\text { GSK3_1 }\end{array}$ & $\begin{array}{l}\text { SATSPSIS } \\
\text { PSISTAMS } \\
\text { SISTAMSS }\end{array}$ & GSK3 phosphorylation recognition site & $\ldots([S T]) \ldots[S T]$ & 0.02679 \\
\hline $\begin{array}{l}\text { MOD__ } \\
\text { NEK2_1 }\end{array}$ & MSSSYP & NEK2 phosphorylation motif & $\begin{array}{l}{[F L M]\left[\left[^{\wedge} P\right]\left[^{\wedge} P\right]([\right.} \\
S T])\left[{ }^{\wedge} D E P\right]\left[^{\wedge} D\right. \\
E]\end{array}$ & 0.009798 \\
\hline $\begin{array}{l}\text { MOD_- } \\
\text { PIKK_1 }\end{array}$ & $\begin{array}{l}\text { GGSTQLQ } \\
\text { SATSPSI }\end{array}$ & $\begin{array}{l}\text { (ST)Q motif which is phosphorylated by } \\
\text { PIKK family members }\end{array}$ & $\ldots([S T]) Q .$. & 0.00923 \\
\hline $\begin{array}{l}\text { MOD_- } \\
\text { ProD } \\
\text { Kin_1 }\end{array}$ & QPVTPGP & $\begin{array}{l}\text { Proline-directed kinase (e.g. MAPK) } \\
\text { phosphorylation site }\end{array}$ & $\ldots([S T]) P .$. & 0.01543 \\
\hline $\begin{array}{l}\text { TRḠ } \\
\text { ENDO } \\
\text { CYTIC_2 }\end{array}$ & $\begin{array}{l}\text { YAGV } \\
\text { YQPM } \\
\text { YGGM }\end{array}$ & $\begin{array}{l}\text { Tyrosine-based sorting signal responsible } \\
\text { for the interaction with mu subunit of AP } \\
\text { (Adaptor Protein) complex }\end{array}$ & Y..[LMVIF] & 0.002587 \\
\hline
\end{tabular}

\begin{tabular}{|c|c|c|c|c|}
\hline $\begin{array}{l}\text { LIG } \\
\text { PAM12_1 }\end{array}$ & $\begin{array}{l}\text { AYPNPQAQY } \\
\text { YQPM }\end{array}$ & $\begin{array}{l}\text { Peptide ligand motif that directly binds to } \\
\text { the MLLE/PABC domain found in poly }(A) \text { - } \\
\text { binding proteins and HYD E3 ubiquitin } \\
\text { ligases }\end{array}$ & $\begin{array}{l}. .[\mathrm{LFP}][\mathrm{NS}][\mathrm{PIV} \\
\text { TAFL].A..(([FY] } \\
\text {.[PYLF])|(W..)). }\end{array}$ & 0.00001 \\
\hline $\begin{array}{l}\mathrm{LIG}_{-} \\
\mathrm{SH}^{3}{ }_{1}\end{array}$ & YPPPPHP & Motif recognized by class I SH3 domains & {$[R K Y] . . P . . P$} & 0.00124 \\
\hline
\end{tabular}


In general, the ELM prediction tool attempts to make as many predictions as possible (Gouw et al., 2017). The results have to be evaluated carefully, as false positive predictions are likely. Each motif pattern is given a probability score, which reflects how probable it is, that the predicted linear motif is a random amino acid sequence rather than a well-defined pattern. $A$ low score indicates a higher probability for the motif to be a true SLiM (Gouw et al., 2017). A cut-off of 0.01 for the probability score was chosen and motif predictions below this threshold are considered to be more likely (TABLE 10, red scores). The results of the ELM prediction are discussed in detail in CHAPTER 4.3.2.

\subsubsection{The VelB intrinsically disordered domain might contain targets for phosphorylation}

The occurrence of posttranslational modifications in intrinsically disordered regions is another way how the functional state, in which a protein can exist in the cell, can be altered. PTMs can determine the variability of intrinsically disordered protein segments, which can influence protein function in different ways; for example as PTM controllable switches, by determining the polyvalent interactions with specific binding partners or the integration of PTM-regulated intra-and intermolecular interactions (Uversky, 2015). Moreover, the occurrence of multiple PTMs can change the net charge which thereby results in the modification of the electrostatic interactions of intrinsically disordered regions (Uversky, 2015).

The VelB intrinsically disordered region of $A$. nidulans contains several tyrosine and serine residues, which might serve as possible phosphorylation targets (FIGURE 37, highlighted in red).

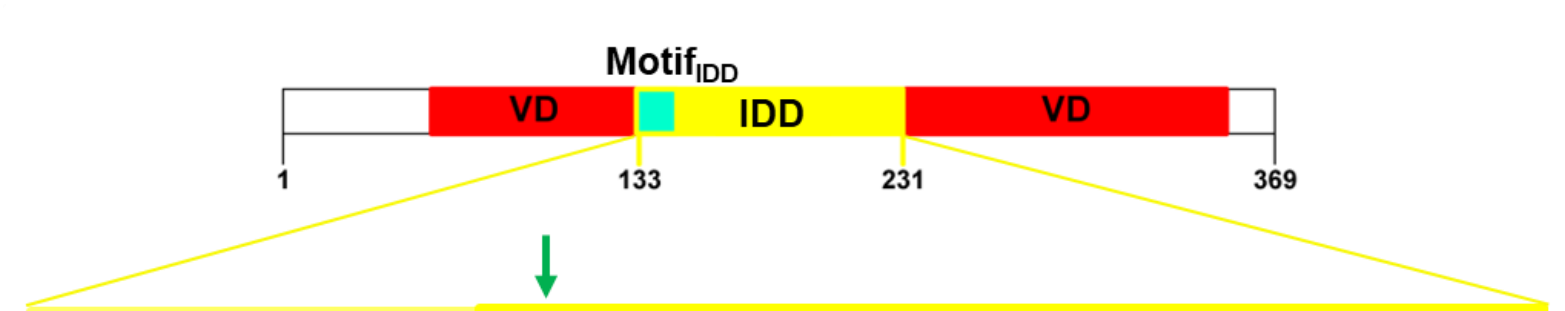

SATSPSISTAMSSSYPPPPHPTSSDYPASYQTNPYGQPVGQPVGQPVGYAG VGNYYGGSTQLQYQNAYPNPQAQYYQPMYGGMAQPQMPAAQPVTPGPG f

FIGURE 37: The VeIB IDD of $A$. nidulans is enriched in proline, serine and tyrosine residues.

Amino acid sequence of the VelB intrinsically disordered domain comprises 99 residues including an 11 amino acid motif (Motif ${ }_{I D D}$, turquoise) conserved among ascomycetes. Proline $(\mathrm{P})$ residues are highlighted in green, serine $(\mathrm{S})$ and tyrosine $(\mathrm{Y})$ residues are highlighted in red. Amino acid residues are given in one letter code. The green arrow indicates a proline-rich sequence within the VelB IDD. 
Furthermore, the VelB IDD of $A$. nidulans is enriched in proline residues (FIGURE 37, highlighted in green), which can be an important feature in molecular recognition, such as intracellular signaling (Kay et al., 2000). Proline residues can serve as binding site for other domains, such as WW domains, which are composed of 40 amino acids and harbor two tryptophan residues (WW), which are spaced around 20 residues apart from each other (Macias et al., 2002). The WW domain recognizes proline-rich sequences and folds in the absence of interaction partners as a stable, triple stranded $\beta$-sheet or forms disulfide bridges (Macias et al., 1996, 2002). The $\underline{\operatorname{Src}}$ homology $\underline{3}$ (SH3) domain also binds to proline-rich ligands, playing a role in many cellular processes, such as regulating protein interactions or changing protein subcellular localization necessary for signaling pathways (Macias et al., 1996; Teyra et al., 2017).

The two bioinformatics tools NetPhos and Scansite are used for proteome-wide identification of phosphorylation sites. It is suggested, that intrinsic disorder in and around potential target sites for phosphorylation is an essential common feature for eukaryotic serine, threonine and tyrosine phosphorylation sites (lakoucheva et al., 2004). Based on this observation, the DISorder-enhanced PHOSphorylation predictor (DISPHOS), which has an enhanced accuracy compared to NetPhos and Scansite, was constructed (lakoucheva et al., 2004). The DISPHOS predictor was employed for the VeIB IDD amino acid sequence and only residues with a score $>0.5$ (which ranges from 0 to 1 ) are considered to be phosphorylated (FIGURE 38). The residues, which are predicted to be phosphorylated, are located in the intrinsically disordered regions of VelB. The velvet domain comprises amino acid 56 to 351. Probably phosphorylated serine resides 18 and 20 (S18, S20) and serine residues 354 and 359 (S354, S359) are part of the presumably intrinsically disordered $\mathrm{N}$ - or C-terminus, respectively (FIGURE 49, DISOPRED prediction of VelB). Serine residues S145, S146, S155 and tyrosine residue Y162 are part of the internal intrinsically disordered domain interrupting the VelB velvet domain (FIGURE 38). These putative phosphosites could be examined in the future research to get information whether they influence the function and structure of the VelB protein.

In summary, the employment of the DISPHOS predictor revealed four putative phosphorylation sites within the intrinsically disordered domain of VelB. ELM search revealed possible linear motifs in the IDD, which might act as functional sites and can be required for protein interaction or posttranslational modifications like phosphorylation or ubiquitination. 


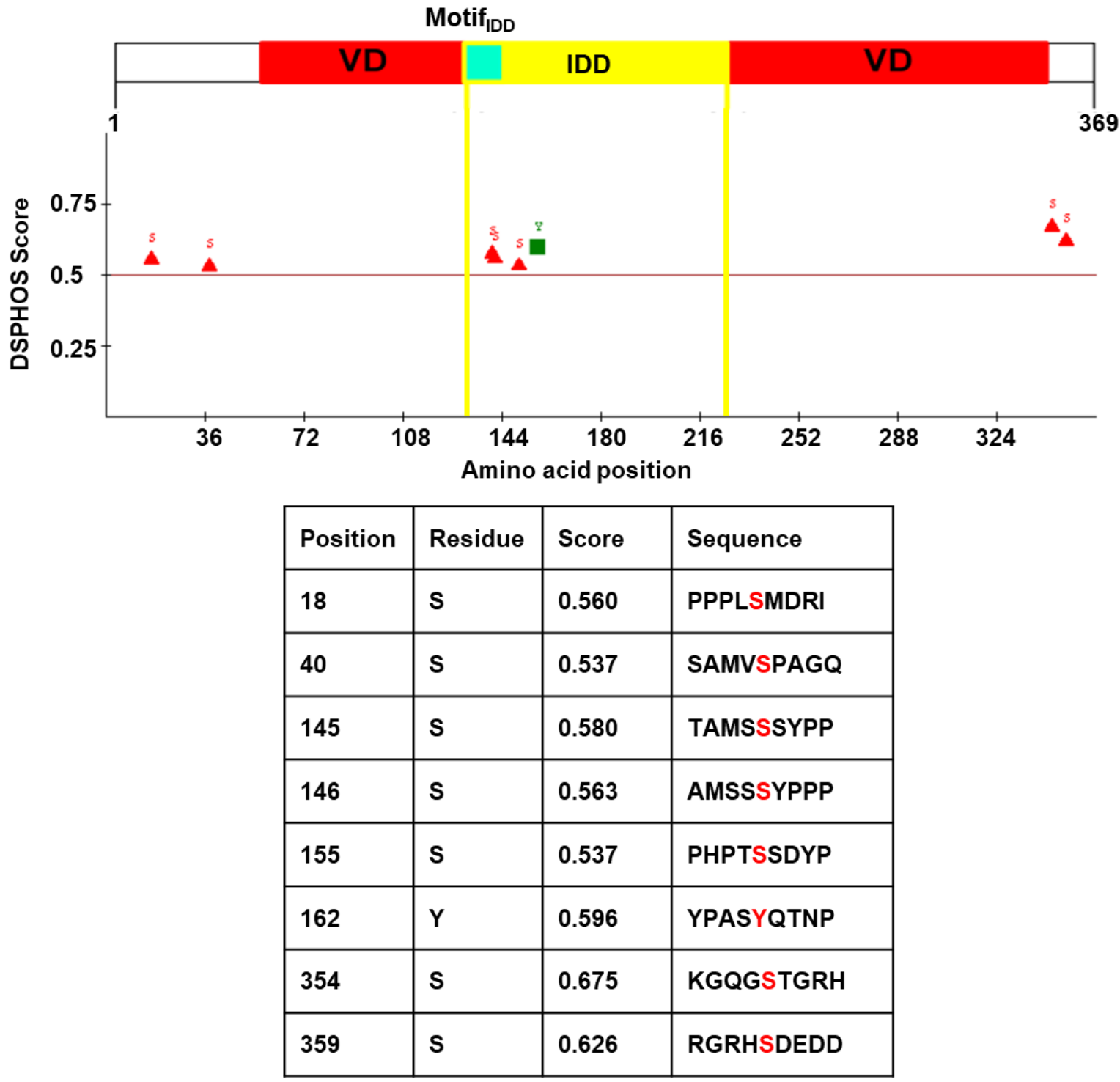

FIGURE 38: Predictions of phosphorylated amino acids of $\boldsymbol{A}$. nidulans VelB.

Employment of the DISPHOS predictor (lakoucheva et al., 2004) revealed eight putative phosphosites within intrinsically disordered segments of VelB. The internal VelB IDD contains four predicted phosphorylated residues. The TABLE depicts the position, residue, DISPHOS score and the surrounding sequence (with the phosphorylated residue highlighted in red). Amino acids with a score $>0.5$ are considered to be phosphorylated. Amino acid residues are given in one letter code. Red triangles indicate putative phosphorylated serine residues (S) and the green square a putative phosphorylated tyrosine residue $(Y)$. 


\subsection{VelC is an auxiliary regulator of development in $A$. nidulans}

\subsubsection{VelC interacts with VosA in A. nidulans during early sexual development}

VelC is beside VeA, VelB and VosA the fourth and last studied member of the velvet domain protein family in A. nidulans (Gerke and Braus, 2014). VelC was described as additional regulator for both asexual and sexual development as deletion of the velC gene leads to increased conidiospore and decreased sexual fruiting body formation (Park et al., 2014). Transcription of velC is detectable in the early stage of sexual development (Park et al., 2014). It was shown that VelC interacts with VosA in vitro and an interaction in early sexual development was suggested, which increases the formation of the VelB-VeA hetero-complex and thereby triggers sexual development (Park et al., 2014). In this study, the velC $\Delta$ phenotype could be complemented by reintroducing the velC::sgfp fusion product (FIGURE 39). This indicates that the C-terminal VelC-GFP fusion protein is functional in A. nidulans.
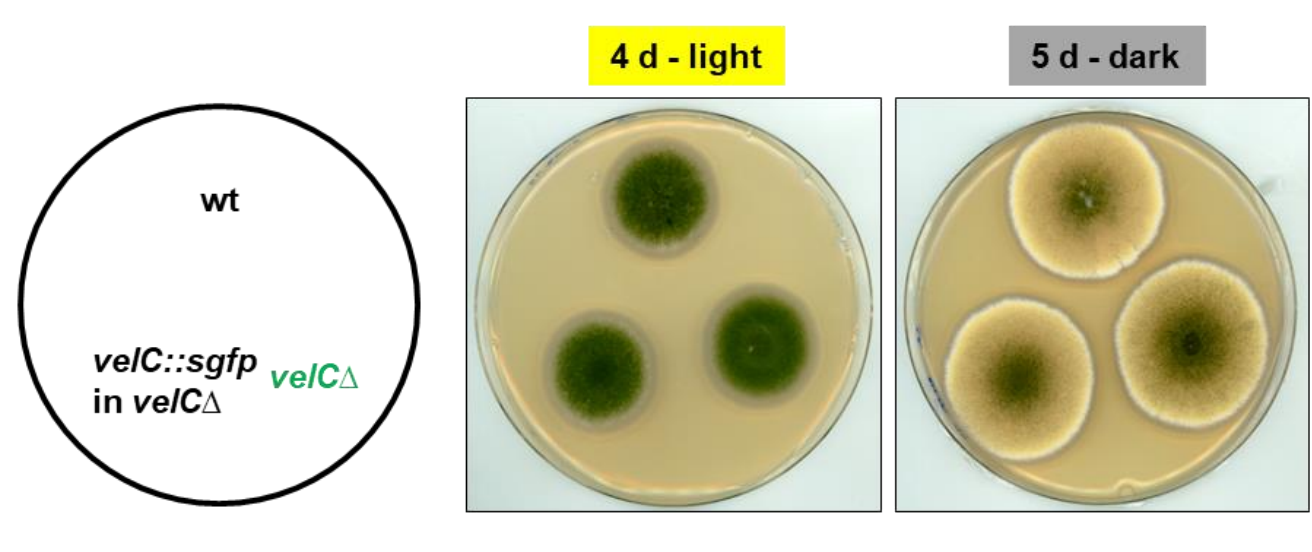

FIGURE 39: The VelC-GFP fusion protein is functional in A. nidulans.

Phenotypes of wildtype (wt), velC deletion (velCs) and complementation (velC::sgfp in velC $\Delta$ ) strain on solid minimal medium grown for 4 days in light and for 5 days in dark.

GFP pull down experiments were conducted in the present study to investigate VelC and putative interaction partners regarding development and secondary metabolism from colonies grown under sexual development promoting conditions. It was examined whether the proposed interaction between VelC and VosA observed during in vitro approaches (Park et al., 2014) also occurs in proteins directly isolated from $A$. nidulans. The strains were grown vegetatively, shifted to solid minimal medium and grown for 48 hours in the dark with limited oxygen supply. The signal for the VelC-GFP fusion protein $(\sim 84 \mathrm{kDa})$ was detected in western hybridization experiments with samples from GFP pull downs of two biological replicates (FIGURE 40). In both cases, a band for the free GFP was detected and especially for the second replicate the signal for VelC-GFP is weaker and several degradation products are visible (FIGURE 40). The VelC protein is unstable and contains a PEST domain at the C-terminus. This region is 
enriched with proline $(P)$, glutamic acid $(E)$, serine $(S)$ and threonine $(T)$ residues and it is hypothesized that the PEST domain is a signal peptide for protein degradation (Gerke and Braus, 2014; Rogers et al., 1986; Spencer et al., 2004).

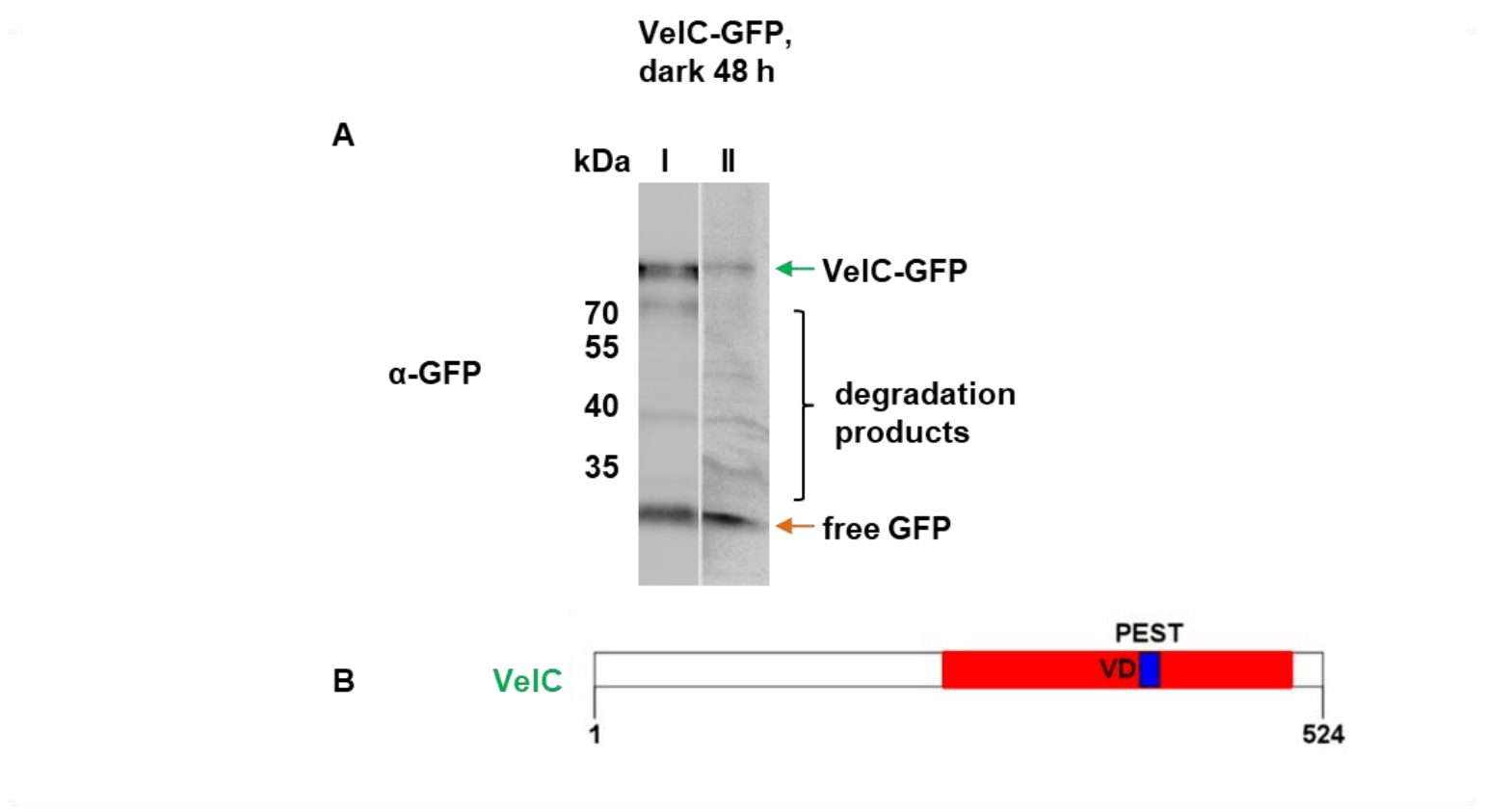

FIGURE 40: VeIC-GFP is unstable and contains a PEST domain.

Western hybridization after GFP pull downs of two biological replicates (I and II) of VelC-GFP fusion protein in $A$. nidulans. Application of the $\alpha$-GFP antibody revealed a signal for VelC-GFP at around $85 \mathrm{kDa}$ (green arrow) and a signal for free GFP (orange arrow). $B$. VelC contains a PEST domain a region enriched with proline $(P)$, glutamic acid $(E)$, serine $(S)$ and threonine $(T)$ residues in the velvet domain (VD), which is typical for unstable proteins.

The instability of VelC-GFP was also reflected in the GFP pull down experiments. VelC as the bait protein was identified in high amounts in the first replicate and several putative interaction partners, not present in the control, were pulled down. In contrast to that, VelC was barely detected for the second replicate (with one unique peptide) and peptides of the GFP tag were present in high amounts, which results in the pull down of several unspecific proteins also identified in the control strain. Due to this, putative interaction partners of the first replicate were filtered and sorted regarding their putative function or localization. VosA was identified as interaction partner of VelC in A. nidulans, which confirms the findings from the yeast-two hybrid assays and in vitro GST pull down experiments from Park and co-workers (Park et al., 2014). Furthermore, proteins from the categories primary metabolism, secondary metabolism, transcription, RNA maturation and processing, translation, protein folding, protein degradation, membrane/cell wall, oxidative stress response, cell compartments and proteins of unknown function were identified. VosA was the only protein pulled down with both, VelB-GFP during vegetative growth and with VelC-GFP during sexual development. The other 28 proteins listed 
in TABLE 11 were only identified in the GFP pull down of the VelC-GFP fusion protein in the dark promoting sexual development.

TABLE 11: Putative interaction partners of $A$. nidulans VelC during early sexual development.

Proteins were identified in one biological replicate with MS/MS counts $\geq 4$, unique peptides $\geq 3$ and LFQ intensity $\geq 20$ but not in the control strain. Sys. Name = systematic name, Std. name = standard name, unchar. = uncharacterized. Descriptions from AspGD are given (Cerqueira et al., 2014).

\begin{tabular}{|c|c|c|}
\hline $\begin{array}{l}\text { Sys. } \\
\text { Name }\end{array}$ & $\begin{array}{l}\text { Std. } \\
\text { name }\end{array}$ & Description \\
\hline AN2059 & VelC & Bait protein \\
\hline \multicolumn{3}{|c|}{ Primary metabolism } \\
\hline AN10399 & unchar. & Putative ortholog of S. cerevisiae AYR1, predicted oxidoreductase \\
\hline AN2343 & unchar. & Putative ortholog of $S$. cerevisiae HBN1, putative nitroreductase \\
\hline AN1534 & unchar. & $\begin{array}{l}\text { Putative ortholog of } A \text {. fumigatus Apt4, putative F1F0-ATPase } \\
\text { complex subunit with a predicted role in energy metabolism; }\end{array}$ \\
\hline AN5586 & unchar. & $\begin{array}{l}\text { Putative ortholog of } A \text {. fumigatus Srb1, putative mannose-1- } \\
\text { phosphate guanylyltransferase with a predicted role in } \\
\text { mannose/mannitol, fructose, and sorbose/sorbitol metabolism }\end{array}$ \\
\hline AN8488 & unchar. & $\begin{array}{l}\text { Putative ortholog of } S \text {. cerevisiae YKT6, SNAP receptor activity, } \\
\text { palmitoyltransferase activity }\end{array}$ \\
\hline AN3829 & unchar. & $\begin{array}{l}\text { Putative ortholog of } A \text {. fumigatus Uga2; putative succinate- } \\
\text { semialdehyde dehydrogenase }\end{array}$ \\
\hline AN2314 & unchar. & $\begin{array}{l}\text { Putative ortholog of } A \text {. niger GbeA, putative 1,4-alpha-glucan } \\
\text { branching enzyme with a predicted role in starch metabolism }\end{array}$ \\
\hline \multicolumn{3}{|c|}{ Secondary metabolism } \\
\hline AN7914 & unchar. & $\begin{array}{l}\text { Putative alcohol dehydrogenase; member of the F-9775 secondary } \\
\text { metabolite gene cluster }\end{array}$ \\
\hline AN7895 & CipB & $\begin{array}{l}\text { Putative oxidoreductase; contains } \mathrm{Zn} \text {-dependent alcohol } \\
\text { dehydrogenase domain }\end{array}$ \\
\hline \multicolumn{3}{|c|}{ Transcription } \\
\hline AN1959 & VosA & $\begin{array}{l}\text { Nuclear protein involved in spore formation and trehalose } \\
\text { accumulation }\end{array}$ \\
\hline
\end{tabular}




\begin{tabular}{|c|c|c|}
\hline \multicolumn{3}{|c|}{ RNA maturation and processing } \\
\hline AN2068 & unchar. & Putative ortholog of $S$. pombe Vgl1, RNA binding effector protein \\
\hline \multicolumn{3}{|c|}{ Translation } \\
\hline AN0314 & unchar. & $\begin{array}{l}\text { Putative ortholog of } S \text {. cerevisiae DPS1, putative aspartyl-tRNA } \\
\text { synthetase with a predicted role in tRNA aminoacylation }\end{array}$ \\
\hline \multicolumn{3}{|c|}{ Protein folding } \\
\hline AN3134 & unchar. & $\begin{array}{l}\text { Putative ortholog of } S \text {. pombe Cct3, role in cellular response to drug } \\
\text { and chaperonin-containing T-complex }\end{array}$ \\
\hline \multicolumn{3}{|c|}{ Protein degradation } \\
\hline AN8054 & Pre6 & Predicted alpha 4 subunit of the $20 \mathrm{~S}$ core proteasome \\
\hline AN2213 & unchar. & Putative ortholog of $A$. flavus proteasome regulatory subunit Rpt2 \\
\hline AN2572 & unchar. & Putative ortholog of $A$. fumigatus DppV, putative dipeptidyl-peptidase \\
\hline \multicolumn{3}{|c|}{ Membrane/cell wall } \\
\hline AN7657 & GelA & $\begin{array}{l}\text { Putative 1,3-beta-transglycosidase with a predicted role in glucan } \\
\text { processing, }\end{array}$ \\
\hline AN3163 & StoA & $\begin{array}{l}\text { Putative stomatin ortholog, predicted to have scaffolding functions in } \\
\text { maintenance of lipid microdomains in membranes, StoA }\end{array}$ \\
\hline \multicolumn{3}{|c|}{ Oxidative stress response } \\
\hline AN10220 & Ccp1 & Putative cytochrome c peroxidase \\
\hline \multicolumn{3}{|c|}{ Cell compartments } \\
\hline AN6980 & Nic96 & Component of the nuclear pore complex \\
\hline AN8874 & unchar. & Putative ortholog of S. cerevisiae Dynamin-related GTPase DNM1, \\
\hline AN6341 & unchar. & $\begin{array}{l}\text { Putative ortholog of } N \text {. crassa coronin } \mathrm{Crn} 1 \text {, predicted role in actin } \\
\text { patch assembly }\end{array}$ \\
\hline \multicolumn{3}{|c|}{ Unknown function } \\
\hline AN7836 & unchar. & Protein of unknown function \\
\hline
\end{tabular}




\begin{tabular}{|l|l|l|}
\hline AN7710 & unchar. & Protein of unknown function \\
\hline AN2954 & unchar. & Protein of unknown function \\
\hline AN1378 & unchar. & Protein of unknown function \\
\hline AN6378 & unchar. & Protein of unknown function \\
\hline AN4127 & unchar. & Protein of unknown function \\
\hline AN2993 & unchar. & Protein of unknown function \\
\hline
\end{tabular}

\subsubsection{Conidiospore formation of $A$. nidulans is delayed in the velB $B^{I D D} / v e / C \Delta$ double mutant}

The formation of different homo- and heterodimers of the velvet domain proteins in $A$. nidulans controls the regulation of development and secondary metabolism, which is influenced by the presence or absence of the intrinsically disordered domain of VelB. VosA interacts with itself and requires an intact VeIB including the IDD for heterodimer formation. Furthermore, VosA interacts with VelC in early sexual development. The phenotype of velvet single deletion strains and $v e / B^{I D D_{\Delta}} /$ velvets double mutants was examined to investigate the outcome of possible and abolished velvet dimer formation regarding the development of $A$. nidulans (FIGURE 41).

The $v e A \Delta$, velB $\Delta$ and $v e l B^{I D D_{\Delta}} / v e A \Delta$ deletion mutants are not capable to form the VelB-VeA heterodimer, as either the $v e A$ or velB gene product is absent. This results in the inability of the fungus to reproduce sexually. Absence of the velB gene product or only of vel$B^{I D D}$ results in the deficiency to form VelB-VosA and VelB-VelB dimers. In contrast to the velB deletion mutant, the velB $B^{I D D \Delta}$ mutant is still able to form VelB-VeA heterodimer. Conidiospore production within high spore densities is drastically decreased in the $v e / B^{I D D_{\Delta}}$ mutant and many aerial hyphae are produced instead. 


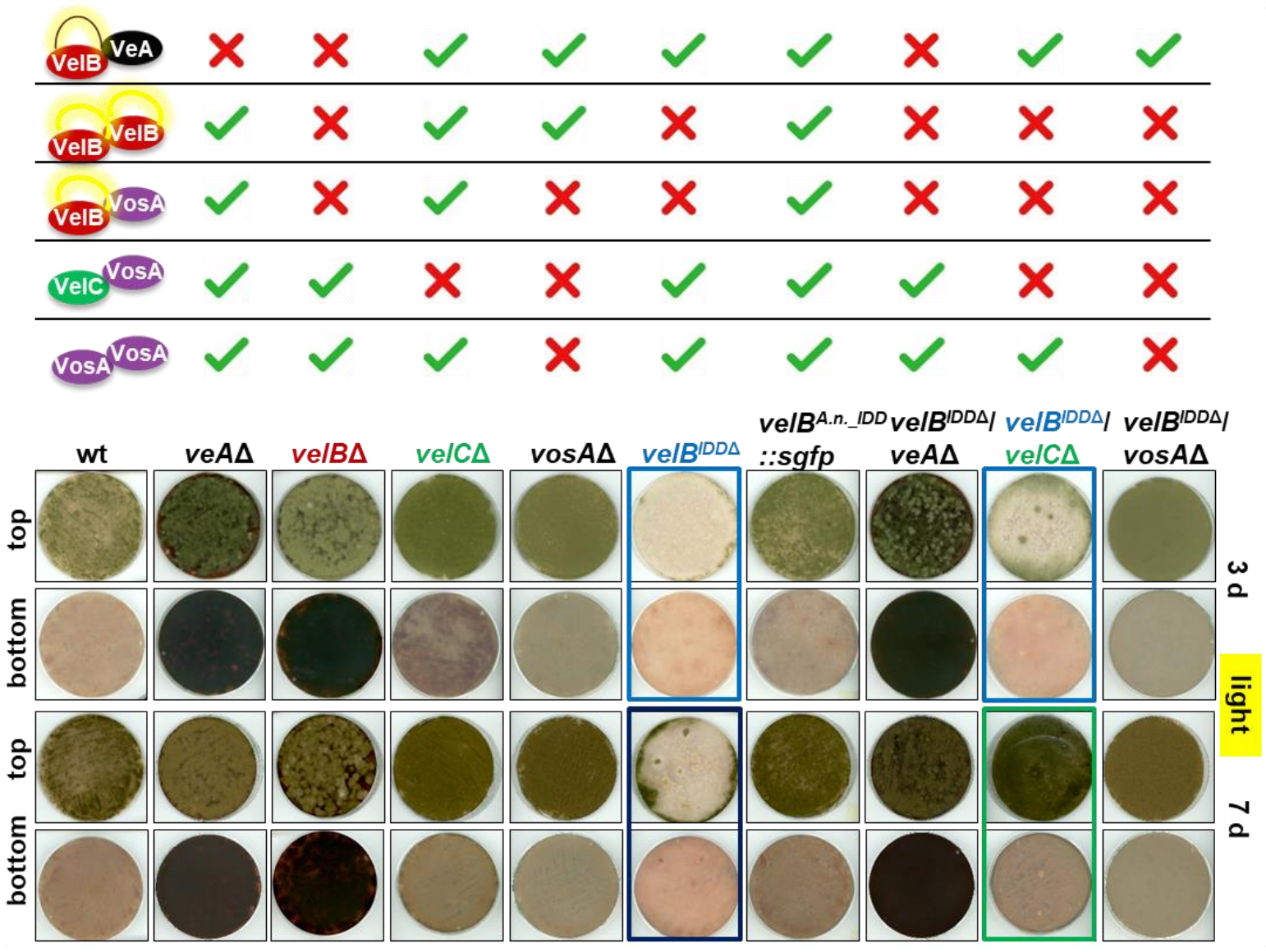

FIGURE 41: Conidiospore formation is delayed in the velB $B^{I D D} / v e / C \Delta$ mutant.

Phenotypes of velvet single deletion and vel$/ B^{I D D} /$ velvet double deletion mutants on solid minimal medium. Strains were grown in the light for 3 and 7 days (d) at $37^{\circ} \mathrm{C}$. The possible velvet dimer formation in the different deletion backgrounds is demonstrated. Many aerial hyphae are built in the vel$B^{I D D \Delta}$ and vel$B^{I D D_{\Delta}} / \mathrm{ve} / C \Delta$ mutant after $3 \mathrm{~d}$ of growth (light blue boxes). Conidiospore production remains reduced in the vel$B^{I D D_{\Delta}}$ mutant after 7 days (dark blue box) and is delayed in the velB ${ }^{I D D_{\Delta}} /$ velC $\Delta$ mutant (green box). $\checkmark=$ present, $\mathbf{X}=$ absent.

Comparison between the velB deletion and the $v e / B^{I D D_{\Delta}}$ deletion mutant reveals that in addition to the VelB-VeA complex, the VelB-VosA and maybe also VelB-VelB dimers are required to form conidiospore-bearing conidiophores within the interconnected and branched mycelium when starting with an inoculum of high spore density. The $v e / B^{I D D_{\Delta}} / v e A \Delta$ and vel $B^{I D D_{\Delta}} / v o s A \Delta$ strains show similar phenotypes to the respective single velvet deletion mutants, veA $\triangle$ and $\operatorname{vos} A \Delta$. VelB-VeA but not the VosA-VosA dimer formation is possible in the velB $B^{I D D_{\Delta}} / \operatorname{vos} A \Delta$ mutant and the phenotype resembles the vosA single deletion mutant. In contrast to that, the $v e / B^{I D D_{\Delta}}$ phenotype predominates in a velB ${ }^{I D D_{\Delta}} / v e / C \Delta$ double mutant strain after three days of growth in light (light blue boxes, FIGURE 41). Contrary to the velB ${ }^{I D D_{\Delta}}$ single deletion mutant (dark blue box), this phenotype, however, is rescued over time in the double mutant (green box, FIGURE 41). VelB-VeA and VosA-VosA dimer formation does not require VelC or the IDD of VelB. Absence of the velC gene product prevents the formation of the VelC-VosA heterodimer and the concurrent lack of the VelB IDD abolishes VelB-VosA and presumably VelB-VelB dimer formation. This suggests that the VosA-VosA dimer represses conidiation at 
early stages of development and the VelC-VosA dimer fulfills this task later during development. The velB ${ }^{I D D_{\Delta}} / \operatorname{vos} A \Delta$ double mutant looks like the $\operatorname{vos} A$ single deletion strain. Neither VosA-VosA nor the VelC-VosA dimer can be formed in this strain as $\operatorname{vos} A$ is absent and asexual development cannot be repressed by these dimers.

Taken together, $A$. nidulans requires the synchronized and accurate formation of the velvet homo- and heterodimers to allow the precise control of development. VelB together with VeA is needed for the induction of sexual development as well as for the early light response. VosAVosA and presumably VelC-VosA inhibit asexual development. The formation of a VelC-VelC homodimer has not yet been described and cannot be excluded. VelB-VosA and supposedly VelB-VelB dimers are required to form conidiophores within the interconnected and branched mycelium at high spore densities.

\subsubsection{Secondary metabolite production is altered in the vel $B^{I D D} / v e / C \Delta$ double mutant}

Deletion of the $v e l B^{I D D}$ in combination with the deletion of $v e / C$ results in delayed conidiospore formation. The decreased production of conidiospores and increased formation of aerial hyphae in a branched mycelium from plated spores with a high concentration, which resembles the $v e / B^{I D D_{\Delta}}$ mutant phenotype, is rescued over time and conidiation is restored. The production of secondary metabolites is tightly interconnected with the developmental programs in $A$. nidulans (Calvo et al., 2002).

Metabolites were extracted from respective strains followed by TLC and detection of the silica plate at $254 \mathrm{~nm}$ and $366 \mathrm{~nm}$ (FIGURE 42, FIGURE 43) (before and after derivatization with $\mathrm{AlCl}_{3}$ ). Investigation of the silica plate at $254 \mathrm{~nm}$ revealed that the production of a so far unidentified secondary metabolite is abolished in the velB ${ }^{I D D \Delta}$ and velB $B^{I D D \Delta} / v e / C \Delta$ mutant strains compared to wildtype after three days of growth in light (white arrows, FIGURE 42). This secondary metabolite remains absent in the vel$B^{I D D \Delta}$ mutant (blue arrow) but is produced in the vel $B^{I D D} /$ velC $\Delta$ mutant strain (yellow arrow) after seven days. This delay in the production of this compound is accompanied by the delay in conidiospore production (FIGURE 41), which indicates a potential role of this secondary metabolite in asexual development. Furthermore the $v e A$ and $v e / B$ deletions strains do not produce this secondary metabolite. It is possible that VelC-VosA acts - under the right conditions - as repressor of the production of this compound. The velB $B^{I D D} /$ velC $\Delta$ mutant strain is restored in the production of this compound after seven days of growth in the light, which might be because its production cannot be repressed by the VelC-VosA heterodimer. 


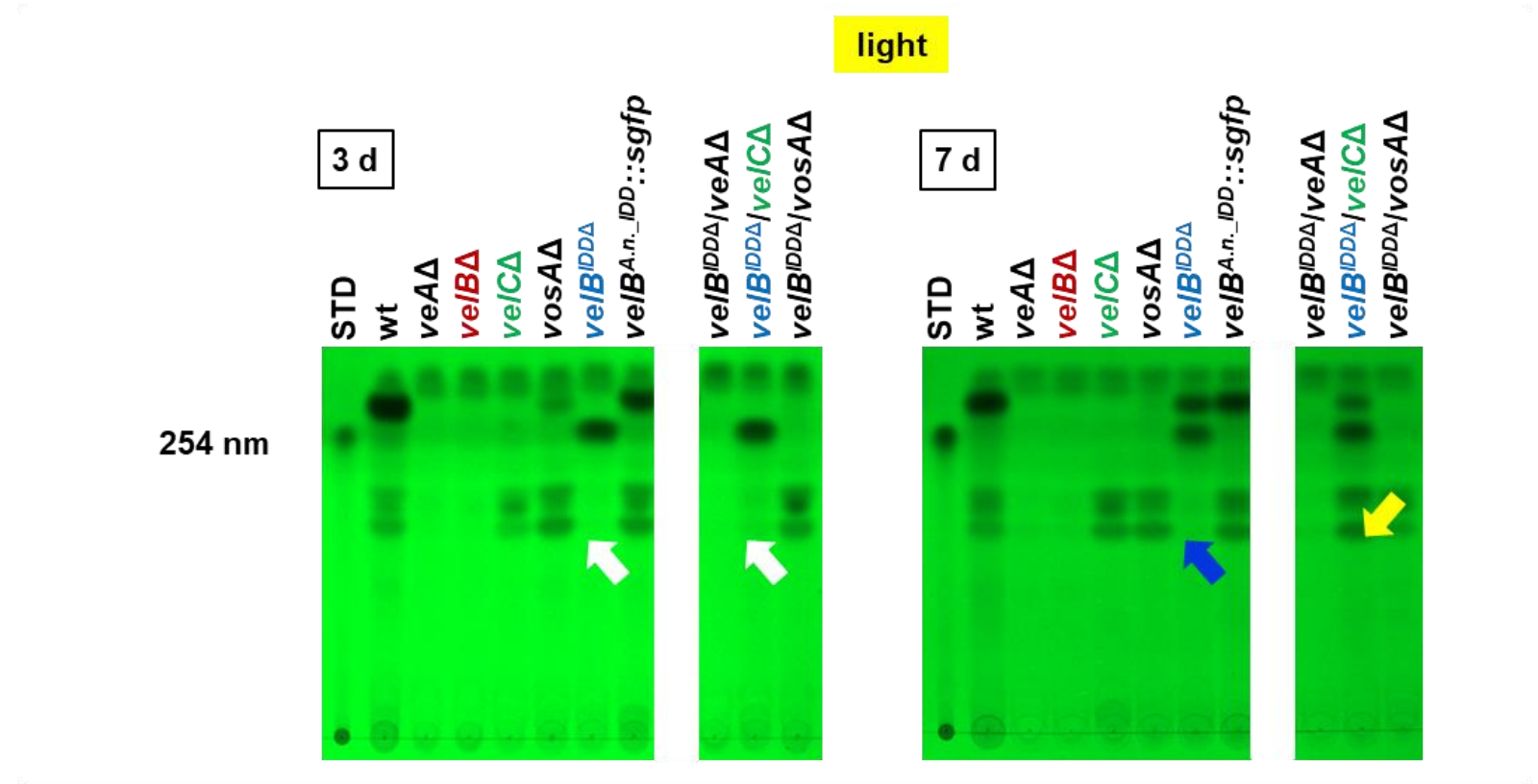

FIGURE 42: The velB ${ }^{I D R} \Delta / v e / C \Delta$ double mutant is delayed in secondary metabolite production.

The signals on the silica plate were detected at $254 \mathrm{~nm}$. The lowest band at $254 \mathrm{~nm}$ (Retention factor $\mathrm{Rf}=0.42$ ) is not present for the vel$/ B^{I D D \Delta}$ mutant and vel$B^{I D D \Delta} /$ velC $\Delta$ double mutant after 3 days of growth (white arrows). This metabolite is visible for the velB $B^{I D D} / v e / C \Delta$ double mutant after 7 days (yellow arrow) but remains undetectable for the velB ${ }^{I D D \Delta}$ single mutant (blue arrow).

The phenotype the velB $B^{I D D} \Delta / v e / C \Delta$ double mutant resembles the velB $B^{I D D \Delta}$ phenotype after three days of growth in light. Deletion of the $v e / B^{I D D}$ results in an increased production of sterigmatocystin compared to wildtype. It was examined whether sterigmatocystin production is also increased in the velB ${ }^{I D D \Delta} / v e I C \Delta$ double mutant. Detection of the TLC silica plate after derivatization revealed that its production is drastically increased not only in the vel$B^{I D D_{\Delta}}$ single but also in the velB ${ }^{I D D} /$ velCA double mutant strain (red arrows, FIGURE 43A). Quantification of the sterigmatocystin signal showed that the production is increased about $500 \%$ compared to wildtype (FIGURE 43B). 
A

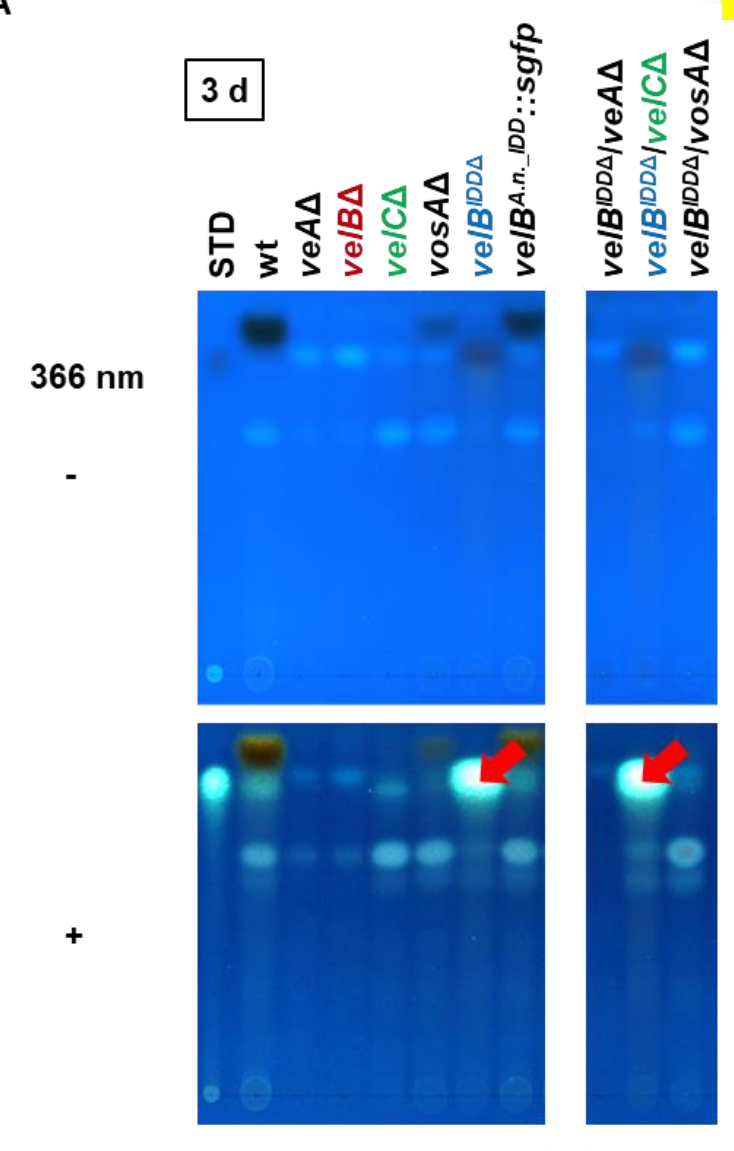

light
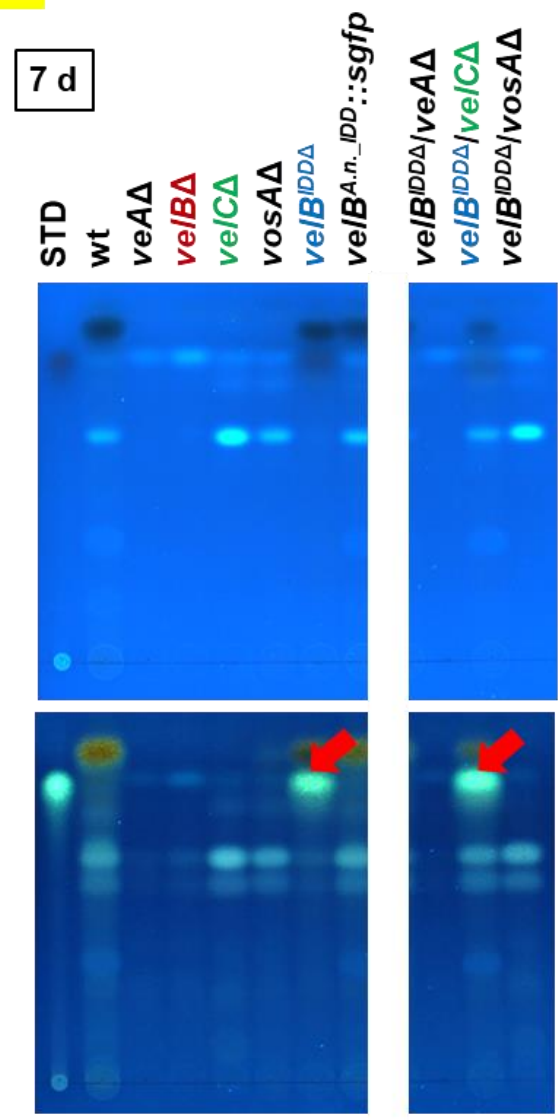

$\square 3 \mathrm{~d}$ - light $\quad \square 7 \mathrm{~d}$ - light

B

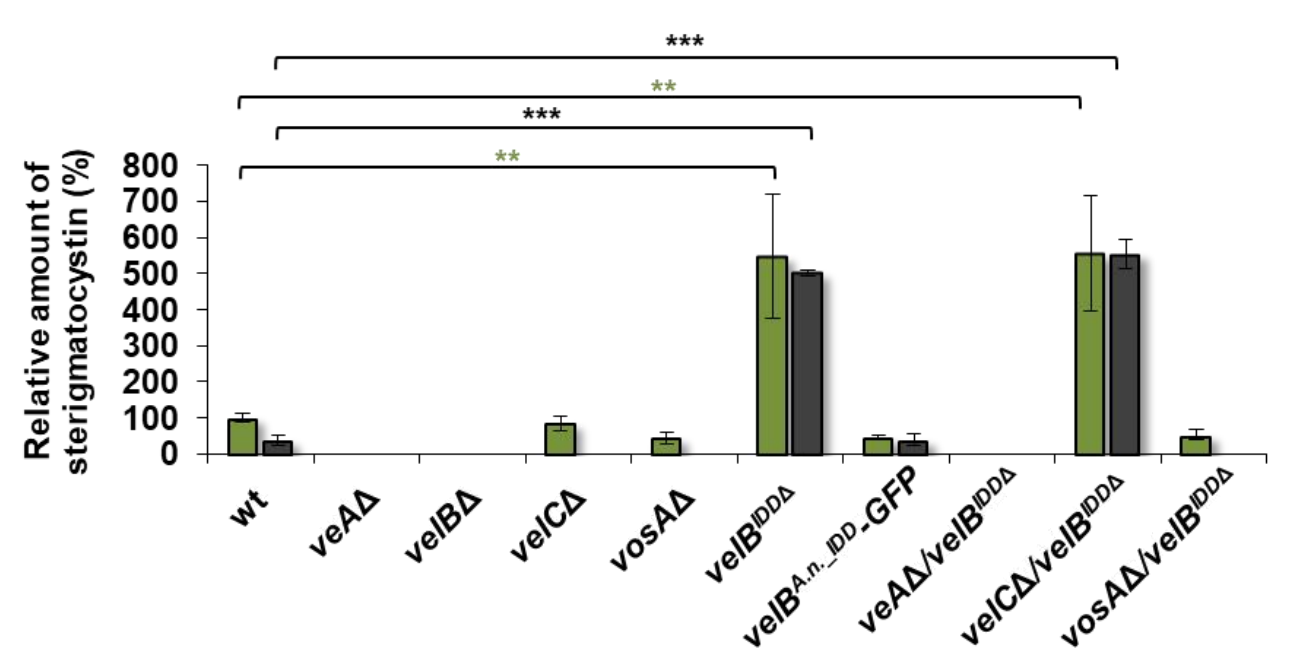

FIGURE 43: Sterigmatocystin production is increased in the vel $B^{I D D \Delta} / v e / C \Delta$ double mutant.

The signals of the same TLC plate from FIGURE 42 were detected at $366 \mathrm{~nm}$ before (-) and after (+) derivatization with $\mathrm{AlCl}_{3}$. Like the velB ${ }^{I D D \Delta}$ mutant also the velB $B^{I D D \Delta} / v e l C \Delta$ double mutant shows an increased production of sterigmatocystin (red arrow). STD = sterigmatocystin standard. Quantification of sterigmatocystin after 3 and 7 days of growth in the light relative to wt. ${ }^{* *}, p<0.01 ;{ }^{* * *}, p<0.005$. Error bars indicate standard error of the mean (SEM) of two biological replicates. $p$-value was calculated with standard deviation. 
This indicates that sterigmatocystin production is indirectly repressed by the VelB-VosA heterodimer. Without this interaction VelB presumably stays in the velvet complex and sterigmatocystin production is increased like in $v e / B^{I D D \Delta}$ and $v e / B^{I D D \Delta} / v e / C \Delta$ mutant strains. In contrast, the velB $B^{I D D_{\Delta}} / \operatorname{vos} A \Delta$ double deletion mutant does not show an increased amount of sterigmatocystin. This implies that either the VosA-VosA homodimer has activation functions or the VelC-VosA heterodimer repression functions regarding sterigmatocystin biosynthesis.

\subsubsection{The VelC-VosA heterodimer is a repressor of asexual development}

VelC interacts with VosA in $A$. nidulans. Deletion of the velC gene results in increased asexual and decreased sexual development (Park et al., 2014), whereas in combination with an absent VeIB IDD conidiation is decreased in early stages of development and restored over time. The influence of deletion of the velC gene combined with the deletion of the complete velB gene was investigated.

Like the velB deletion mutant, the velB $\Delta / v^{2} / C \Delta$ double deletion mutant is unable to form the VelB-VeA heterodimer and is therefore abolished in the formation of sexual fruiting bodies in contrast to wildtype and the velC single deletion mutant (white arrows, FIGURE 44). The vel $B^{I D D_{\Delta}} /$ velC $\Delta$ double mutant resembles the velB $B^{I D D_{\Delta}}$ when inoculated as a single spot. The colonies look less green and the agar is less pigmented compared to the wildtype. In contrast, the colony of the double mutant looks greener and is surrounded by a yellow ring in the agar in both dark and light, additional to the brown-red pigments, which are also released by the velB single deletion mutant (FIGURE 44). This indicates that secondary metabolites, which are not produced in the wildtype cells, are built in the velB $\Delta / v e l C \Delta$ double mutant. Phenotypic analyses already suggested an increased conidiation in the velB $\Delta / v e l C \Delta$ double mutant (FIGURE 44). Therefore, conidiospore production was quantified and the conidiospore viability was investigated (FIGURE 45). 

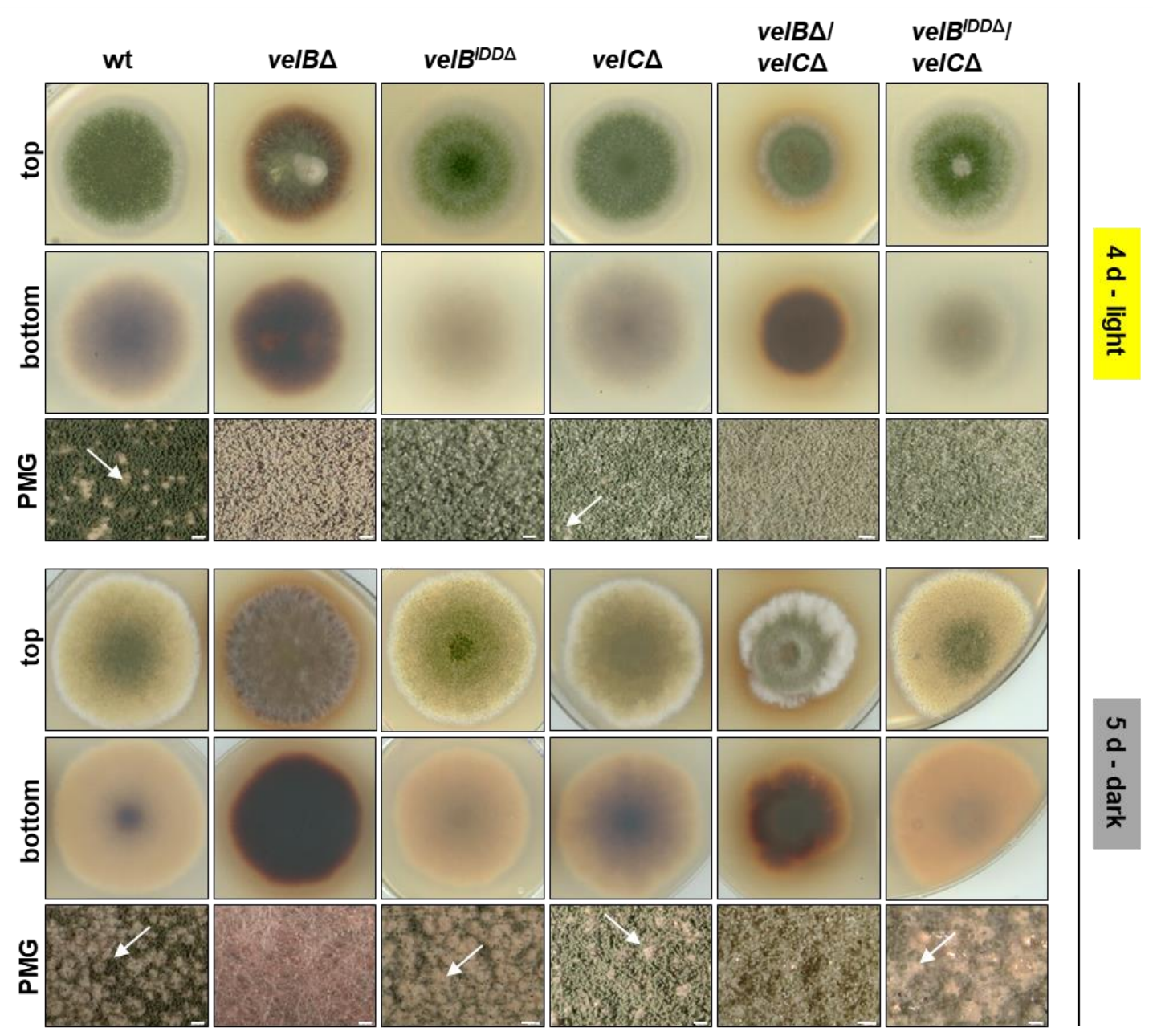

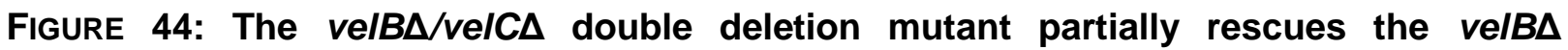
phenotype.

Phenotypes of wildtype (wt), velB deletion (velB $\Delta$ ), velC deletion (velC $\Delta$ ) and the velB/velC double deletion (velB $\Delta /$ velC $\Delta$ ) strains after 4 days of growth in light and 5 days of growth in dark. $\mathrm{PMG}=$ Photomicrograph, Scale bar $=200 \mu \mathrm{m}$, white arrows indicate cleistothecia.

The $v e l B / \Delta v e l C \Delta$ double deletion mutant produces increased amounts of conidiospores relative to wildtype. After five days of growth in the light, promoting asexual development, conidiospore production is increased to $300 \%$ in the velC $\Delta$ mutant and significantly increased to $400 \%$ in the velB $/$ velC $\Delta$ double deletion mutant, compared to wildtype (FIGURE 45A). Conidiospore production is rescued in the velB deletion mutant when the velC gene is deleted as well. In addition, influence on the survival of conidia was examined. Around $50 \%$ of the spores inoculated for the wildtype and velC deletion mutant formed colonies after 10 days. In contrast, only $14 \%$ of spores of the velB single and $20 \%$ of spores of the velB $\Delta / v e / C \Delta$ double deletion mutant survived and formed new colonies.

In summary, deletion of the velC gene in the velB $\Delta$ background rescues the decreased conidia production, but not the deficiency in the viability of conidiospores. This supports that the VelCVosA heterodimer, which formation is disabled upon velC deletion, acts as additional repressor of conidiospore formation. 
A

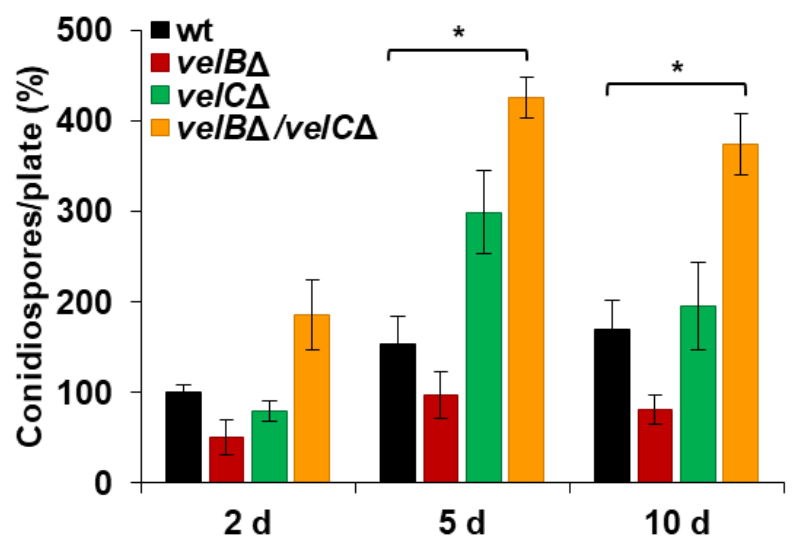

B

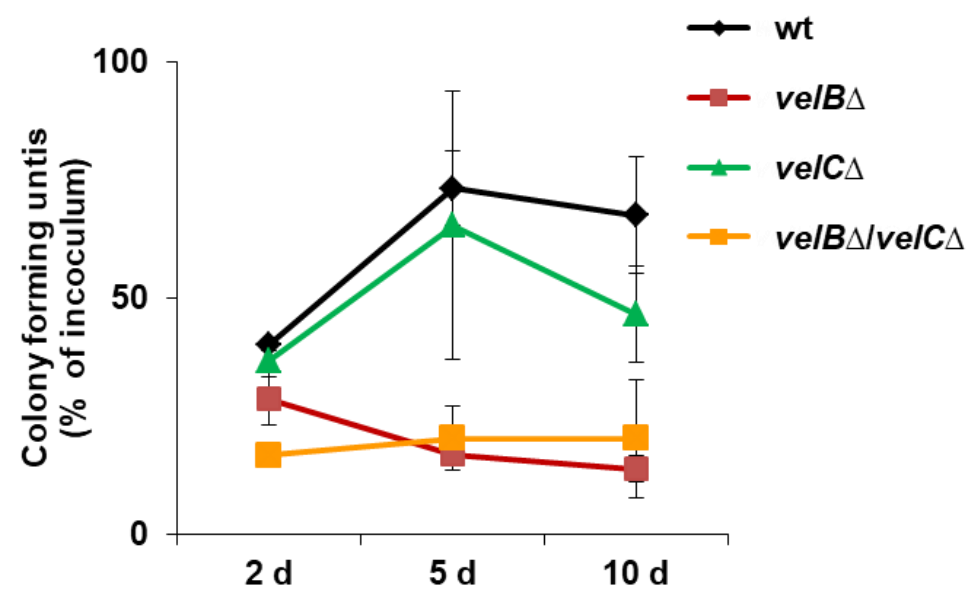

FIGURE 45: The velB/velC double deletion mutant produces more conidiospores than the wildtype.

Quantification of conidiospores after 2, 5 and 10 days (d) of growth in light at $37^{\circ} \mathrm{C}$. Error bars indicate standard error of the mean (SEM) of three biological replicates. P-value was calculated with standard deviation. ${ }^{*}, \mathrm{p}<0.05$. B. Spore viability is decreased in the $v e l B /$ velC double deletion mutant. After collection of the spores after 2, 5 or 10 days, 200 spores were plated and the surviving colonies were counted after 2 days.

Velvet domain proteins are required for the orchestration of development and secondary metabolism and deletions of the corresponding genes have severe influences. In the present study, it was investigated whether the presence of all four velvet genes is essential in A. nidulans and a velvet quadruple deletion mutant (velC $\Delta / \operatorname{vos} A \Delta /$ velB $\Delta / v e A \Delta)$ was constructed. This mutant is viable and abolished in the production of sexual fruiting bodies and drastically decreased in conidiospore formation (FIGURE 46). Furthermore, the colony is smaller and the agar pigmented in a dark red. This shows that all four velvet domain proteins are required for an accurate control of development and secondary metabolism but are not essential in A. nidulans. 


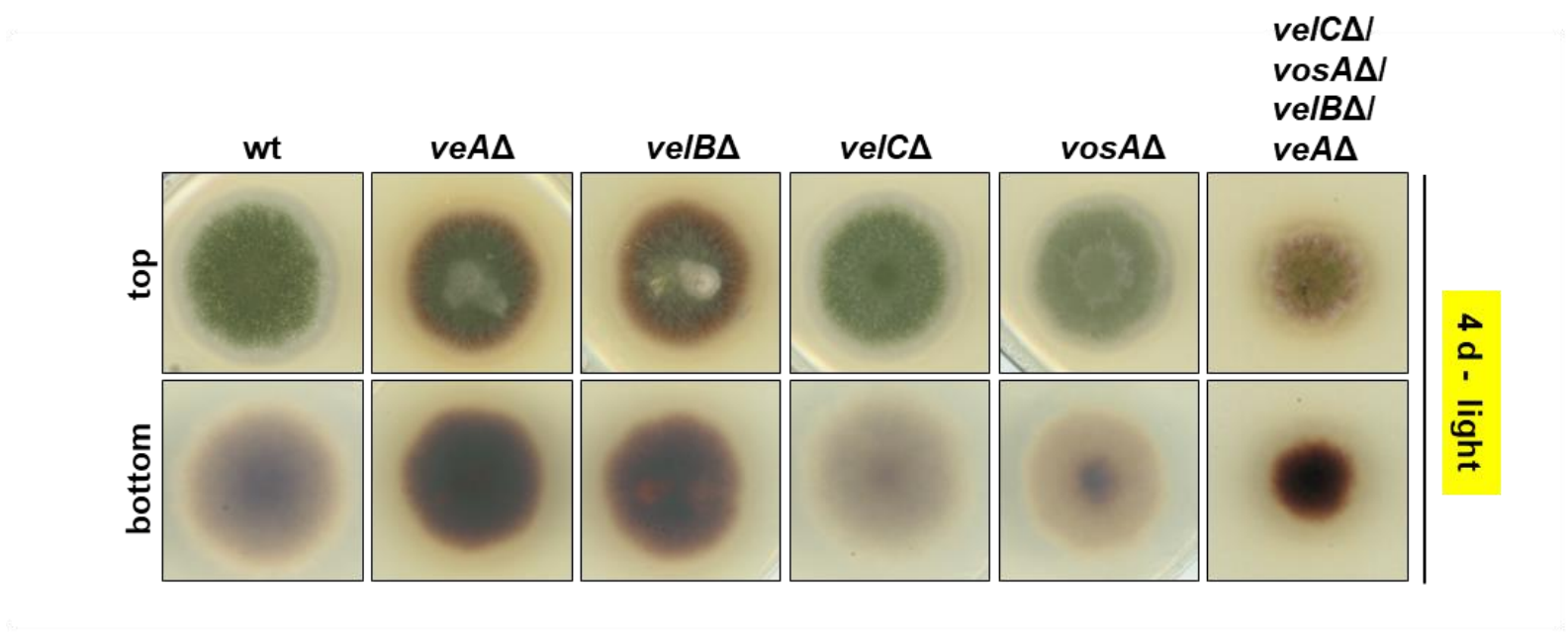

FIGURE 46: $A$. nidulans is viable without the four velvet encoding genes.

Phenotypes of wildtype (wt), velvet single deletion (veA $\Delta$, velB $\Delta$, velC $\Delta$, vos $A \Delta$ ) strains and velvet quadruple deletion mutant (velC $/$ vos $A \Delta /$ velB $\Delta / v e A \Delta)$ grown for 4 days in light at $37^{\circ} \mathrm{C}$.

In conclusion, the presented study revealed that selective dimer formation of the velvet domain proteins is required for the coordination of development and secondary metabolism in A. nidulans. VelB is the only protein of the four velvet domain regulators with an internal intrinsically disordered domain, which interrupts the velvet domain. VelB as a hub in protein interaction networks forms dimeric complexes with $\mathrm{VeA}$, VosA and presumably with itself. The VelB IDD is dispensable for VelB-VeA heterodimer formation but required for the interaction with VosA. Homodimer formation is also abolished in vitro in the absence of the VelB IDD. Furthermore, the VeIB IDD is a potential target for degradation, as its presence destabilizes the VelB protein, which is also negatively influenced by Fbx18.

Besides its interaction with VelB during vegetative growth, VosA also interacts with VelC during early sexual development in $A$. nidulans. The VelC-VosA heterodimer presumably acts as additional repressor of asexual development. Taken together, a balanced velvet domain protein dimer and complex formation, which is spatially and timely regulated, is required for appropriate conidiospore formation and regulated secondary metabolite production. 


\section{Discussion}

\subsection{Insertion of an intrinsically disordered domain into the VelB velvet domain results in a novel layer of control of the dimerization potential of a fungal transcription factor}

Environmental changes trigger the decision for the appropriate developmental program and production of secondary metabolites in $A$. nidulans. The presence or absence of light and the availability of oxygen represent two main stimuli, which promote, especially under laboratory conditions, either asexual or sexual development in this fungal model organism. Genetic networks represent the assortment of specific molecular regulators and include the control by DNA binding proteins, which can act as homo- or heterodimers. The velvet domain represents a conserved fungal DNA binding and dimerization domain of approximately 150 amino acids, which is reminiscent to the Rel homology domain of NF-KB in the mammalian immune and infection response (Ahmed et al., 2013). Aspergilli possess four members of velvet family proteins (VeA, VelB, VelC and VosA), which form homo- or heterodimers and are required to control development coordinated with the appropriate interconnected secondary metabolism and spore viability (Gerke and Braus, 2014).

VeA interacts with several epigenetic methyltransferases and enhances the transport of VelB as a VeA-VelB heterodimer into the nucleus (Bayram et al., 2008; Sarikaya-Bayram et al., 2015). VelB is also part of the VelB-VosA complex promoting spore viability. In contrast to the velvet domain of other velvet proteins, the $A$. nidulans VelB velvet domain is interrupted by an intrinsically disordered domain of 99 amino acids, which is conserved among Aspergilli (FIGURE 4). The amount of velvet domain proteins and the ratio of the possible complexes are controlled by protein synthesis and degradation. The regulation of cellular amounts of VelB by destabilizing the protein is one mechanism to control its abundance and distribution to different complexes within the fungal cell. Furthermore, the VelB intrinsically disordered domain could serve as an additional control mechanism to support selective heterodimer formation in A. nidulans.

Hub proteins in protein interaction networks often contain segments of intrinsic disorder. These enable them to form complexes with different partners, what gives them the possibility to coordinate and regulate several cellular activities (Hu et al., 2017). VelB interacts with VeA, VosA and forms homodimers with itself and is a regulator of sexual and asexual development and interconnected secondary metabolism (Bayram et al., 2008; Park et al., 2012b). Interaction partners of VelB with and without IDD were compared and GFP pull down experiments of vegetatively grown mycelium were conducted. VeA was identified as interaction partner for the full length VeIB as well as for VelB without the IDD (TABLE 7). This indicates that 
the VelB IDD is dispensable for VelB-VeA heterodimer formation. The formation of the VelBVeA-LaeA complex also still seems to be possible, as LaeA was also identified (below threshold) in both GFP pull downs. VeA localization is dependent on light where it is distributed in the cytoplasm and in the nucleus, but in the dark VeA subcellular localization is shifted towards the nucleus (Stinnett et al., 2007). The localization of VelB is independent of light but the nucleic VelB-GFP signal is weaker (but not completely abolished) in a veA deletion background grown in the dark, compared to wildtype (Bayram et al., 2008). This indicates that VeA enhances the transport of VelB into the nucleus but it is also possible that other proteins shuttle VelB into the nucleus as well. VeA-GFP is also localized in nuclei in vegetative hyphae in submerged cultures in the light (C. Meister, p.c.), which suggests that VeA might support nuclear entrance of VelB under these conditions as well. Taken together, the subcellular localization of VelB as well as complex formation with VeA is independent of the VelB IDD in vegetative hyphae (FIGURE 15).

It was shown that VelB interacts with VosA in the nucleus (Sarikaya-Bayram et al., 2010). The current examinations present that the absence of the VeIB IDD abolishes interaction with VosA, which was only pulled down with VelB-GFP but not with VelB ${ }^{\operatorname{IDD} \Delta_{-} G F P}$ in immunoprecipitation experiments. Co-immunoprecipitation investigations supported that the VeIB IDD is required for a stable VelB-VosA heterodimer formation (FIGURE 17). The crystal structure of the VelB-Vos $A_{1-190}$ heterodimer lacks the VelB IDD, which was presumably cleaved off due to proteolytic removal (Ahmed et al., 2013). A truncated version of VosA, encompassing the velvet domain from residue 1 to 190 (Vos $\left.A_{1-190}\right)$, and the full length VelB protein were used for dimer formation and crystallization (Ahmed et al., 2013). The present study reveals that the VosA velvet domain is sufficient to form a heterodimer with VelB independently of the IDD in vitro (FIGURE 18). Previous studies from Bayram and co-workers also showed in Yeast-two-hybrid $(\mathrm{Y} 2 \mathrm{H})$ assays, that the $\mathrm{N}$-terminus of VosA (residue 1-239), which harbors the velvet domain, is sufficient to bind to VelB, whereas the VosA C-terminus (211-430) fails to interact with VelB in vitro (Bayram et al., 2008). Truncated protein versions of VelB, containing only the $\mathrm{N}$ - or the C-terminus of the protein, were also tested regarding the interaction with the full length VosA protein and both VelB versions interact with VosA (Bayram et al., 2008). VelB-N, spanning residue 1 to 231, and VelB-C, encompassing residue 142 to 369 , both still contain (most of) the intrinsically disordered domain, which is located from amino acid 133 to 231 . Anyhow it is not clear whether the folding of truncated velvet domain protein versions agrees in vivo with the observations made in vitro. Altogether, the results indicate that the intrinsically disordered domain of VelB is required for the stable complex formation with VosA in $A$. nidulans but dispensable for interacting with the velvet domain in the truncated $\operatorname{Vos}_{1-190}$ in vitro. This indicates that an additional interacting protein(s) might bind to the VelB IDD or that the VeIB IDD might be modified by posttranslational modification in vivo. 
Split-YFP experiments suggest VelB homodimer formation in A. nidulans (Sarikaya-Bayram et al., 2010), which was not indicated in the $\mathrm{Y} 2 \mathrm{H}$ experiments in vitro (Bayram et al., 2008). In this work it was confirmed with in vitro experiments that the VeIB IDD is required for homodimer formation (FIGURE 20). VelB-His and VelB ${ }^{I D D} \Delta_{-}$His fusion proteins recombinantly expressed in E. coli were purified by affinity chromatography and further analyzed regarding dimerization by size exclusion chromatography. The majority of the VeIB-His protein elutes as a monomer. In contrast, just a small UV maximum peak was detected for the dimeric VelB-His. This indicates that only a small subpopulation of VelB forms the homodimer in vitro and was therefore probably not detected in the $\mathrm{Y} 2 \mathrm{H}$ experiments in vitro. velB is expressed under the inducible nitrate promoter during split-YFP, which might have increased the chance to visualize the VelB homodimer. VelB homodimers might be present in higher or lower subpopulations or the homodimer could also be a resting complex of VelB to prevent the interaction with other proteins in vivo in $A$. nidulans. Size exclusion chromatography revealed that the VelB ${ }^{\operatorname{DDD} \Delta_{-}}-\mathrm{His}$ fusion protein only elutes as a monomer but no dimeric protein was detected. This suggests that the VeIB IDD is, besides complex formation with VosA in A. nidulans, also required for homodimer formation in vitro.

\subsubsection{A molecular mechanism might enable different VelB complex formations in the nucleus}

The balanced homo- and heterodimer formation between VelB with itself, VosA and VeA requires the VeIB IDD and the equilibrium is moved towards VelB-VeA dimer formation in its absence. This suggests that the VeIB IDD is involved in a molecular mechanism, which enables the transfer of VelB from the VelB-VeA heterodimer to form the VelB-VosA heterodimer and VelB homodimer in the nucleus in wildtype conditions. One possible explanation approach could be the masking of the VeIB IDD in the nucleus to prevent competition of VosA for dimerization with VelB (FIGURE 47). This masking could also lead to a conformational change of the VeIB IDD, which disables VosA to bind.

Different mechanisms regarding conformational alterations of the heterogeneous spatiotemporal structure of proteins, which are intrinsically disordered or contain disordered regions, are known. These unstructured parts of a protein can be described as a modular collection of foldons (independent foldable parts of a protein): inducible foldons, semi-foldons, non-foldons and unfoldons (Uversky, 2013, 2015). Alterations of the conformational state of these foldons can occur upon various factors, such as interacting with another protein, posttranslational modification or changing of other intra-or extracellular factors.

Unfoldons are characterized as structured regions with a dormant disorder within a protein. They have to be transiently awakened to undergo an order-to-disorder transition to make the protein functional. Different factors, such as $\mathrm{pH}$, temperature, redox potential, light, mechanical 
force, interaction with ligands or PTMs can result in unfolding a structure of a folded protein (Jakob et al., 2014; Uversky, 2013). One example is the acidification-induced activation of the bacterial chaperone HdeA. The dimeric HdeA is folded and inactive at a neutral $\mathrm{pH}$, but the protein partially unfolds at acidic $\mathrm{pH}$ and dissociates into chaperone-active monomers (Hong et al., 2005). Disordered regions within proteins, which do not fold at all, are defined as nonfoldons, as binding of disordered protein molecules is not automatically followed by disorderto-order transition (Sigalov et al., 2004). For example, the interaction between glycine-rich intrinsically disordered domains of human Sp1 and TAF4 transcription factors is not accompanied by significant conformational changes (Hibino et al., 2016). Also the homodimer formation of Sp1 does not result in a disorder-to-order transition of the ID domain (Hiramatsu et al., 2012). Partially folded segments of proteins with transient residual structure, which are always in a semi-folded state, are categorized as semi-foldons (Sigalov et al., 2004; Uversky, 2013). Inducible foldons within disordered domains can change the conformational state of the protein by folding at interaction with specific binding partners. The tumor suppressor protein and transcription factor p53 contains for example an N-terminal intrinsically disordered region, which folds upon binding to the TAZ2 domain of the co-activator p300 (Wells et al., 2008). Another example is the intrinsically disordered interaction domain of the transcription factor STAT2, which undergoes a disorder-to-order transition upon binding to the TAZ1 domain of the CREB-binding protein CBP (Dyson and Wright, 2016).

In the present study, the influence of the $A$. nidulans VeIB IDD regarding selective dimer formation with velvet domain proteins was investigated and following molecular mechanism is proposed (FIGURE 47). The interaction of VelB with VeA is independent of the IDD and the VelB-VeA heterodimer is considered as "default setting" for VelB dimers in the fungal cell. VelB IDD-dependent activators or inhibitors might be required to trigger or prevent the formation of the different complexes in the cytoplasm or the nucleus, respectively (FIGURE 47). SarikayaBayram and co-workers showed in split-YFP experiments that VelB-VosA interaction is restricted to the nucleus (Sarikaya-Bayram et al., 2010). Therefore, it could be concluded here, that formation of the VelB-VosA heterodimer is inhibited in the cytoplasm in a VelB IDDdependent manner, for example by another interacting protein or modification of the VeIB IDD by a posttranslational modification (FIGURE 47). This in turn enables the nuclear entry of VelB, supported by VeA in a complex with the a-importin KapA (Bayram et al., 2008). Split-YFP experiments also indicate the occurrence of cytoplasmic as well as nuclear subpopulations of the VelB-VelB homodimer (Sarikaya-Bayram et al., 2010). 


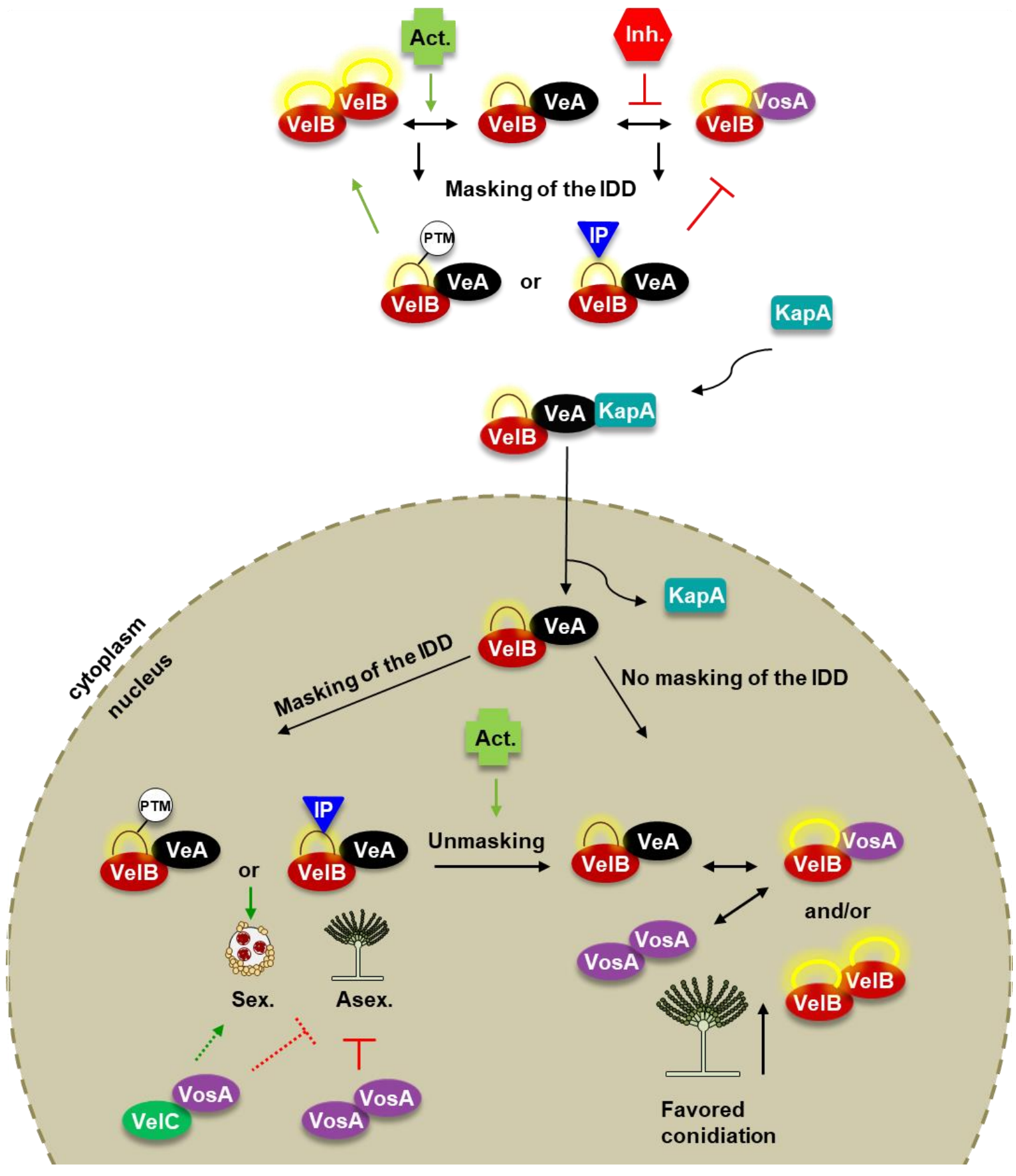

FIGURE 47: Selective heterodimer formation is supported by the VelB IDD in A. nidulans. VeIB IDD-dependent activators (Act.) or inhibitors (Inh.) might be required to induce or block the formation of the different complexes in the cytoplasm or the nucleus. VeA supports nuclear entry of VelB in a complex with the a-importin KapA. The VelB IDD could be masked by another interacting protein (IP) or by a posttranslational modification (PTM) to remain as the VelB-VeA heterodimer and prevent complex formation with VosA and/or VelB itself. Enhanced VelB-VeA formation, which induces sexual development, can lead to increased VosA-VosA homodimer and maybe VelC-VosA heterodimer formation, for their part repressing asexual development and VelC-VosA presumably also supporting sexual fruiting body formation. Accessible VelB IDD or unmasking of the masked VeIB IDD enables VosA to compete for complex formation with VelB. The dimer formation ratio is shifted towards VelB-VosA and VelB-VelB and less VosA-VosA and presumably VelC-VosA dimers are formed, what in turn favors conidiospore formation. Asex. = Asexual development, Sex. = Sexual development. 
The present study shows that the homodimer formation requires the VeIB IDD in vitro. This indicates the presence of an IDD-dependent activator, which allows the formation of the VelB-VelB homodimer in the cytoplasm or in the nucleus. The VelB-VelB homodimer in the cytoplasm might serve as resting complex to hinder nuclear entry of VelB together with VeA.

Velvet domain proteins are fungal specific transcription factors and the formation of different hetero- and homodimers are required for the control of appropriate target genes in the nucleus. After nuclear entrance of VelB, especially competition of VeA and VosA for VelB seems to be required for the cellular response to diverse external and internal stimuli. The masking of the VeIB IDD by another interacting protein or by a posttranslational modification might be a possible mechanism to keep VelB in a complex with VeA, which is required for the initiation of sexual fruiting body formation (FIGURE 47). This could be enabled by a conformational change of the VeIB IDD upon masking, which in turn strengthens the interaction with VeA. This could on the other hand enhance VosA-VosA and presumably VelC-VosA complex formation, as VosA does not compete for interaction with VelB when the IDD is masked. VosA-VosA (and presumably VelC-VosA) represses asexual development and VelC-VosA might support sexual development (Ahmed et al., 2013; Ni and Yu, 2007; Park et al., 2014).

However, under certain circumstances VosA competes for dimerization with VelB and thereby alters the ratio of velvet domain protein dimers (FIGURE 47). This would require an accessible VeIB IDD or unmasking of a masked or modified VeIB IDD. The ratio of velvet domain protein dimers would be shifted towards VelB-VosA and VelB-VelB, which in turn would result in less VosA-VosA and presumably VelC-VosA dimers, thereby favoring conidiospore formation.

\subsection{The amino acid sequence of the VelB intrinsically disordered domain evolved rapidly in filamentous fungi}

Amino acid sequences of structured domains can change during evolution but often the structure remains and conclusions about the function of proteins can be drawn. In contrast, regions, which are intrinsically disordered (ID), evolve rapidly. This makes it increasingly difficult to identify homologous regions and to transfer functional information between homologs and predict functions of intrinsically disordered domains (Brown et al., 2009). The velvet domain is a good example for these evolutionary relationships. At first, no sequence similarity to any known protein domain could be identified for the velvet domain. This changed when the structural information of the velvet domain was available. Crystal structure analysis of the VelB-VosA heterodimer and employment of the DALI program revealed that the Rel homology domain of the mammalian transcription factor NF-kB has the most similar protein structure to the velvet domain of VosA (FIGURE 5) with an amino acid sequence identity of $13.7 \%$ (Ahmed et al., 2013). This shows that the amino acid sequences of theses domains 
altered significantly during evolution but the structural similarity remained. The similarity between the Rel homology domain and the velvet domain could be due to independent convergent evolution but it is also possible that these proteins originate from a common ancestor (Ahmed et al., 2013; Gerke and Braus, 2014). The velvet domain is highly conserved in the fungal kingdom and VelB is the only protein of the velvet family, which domain is interrupted (Ahmed et al., 2013; Bayram and Braus, 2012). The amino acid sequence of the VeIB IDD is conserved among Aspergillus species and an 11 amino acid serine-rich region at the N-terminus is shared in ascomycetes like Neurospora crassa or Verticillium dahliae (FIGURE 13). The presence of the VeIB IDD is maintained in other filamentous fungi as well, like in the basidiomycetes Coprinopsis cinerea, Laccaria bicolor or Cryptococcus neoformans, but the amino acid sequence differs considerably (FIGURE 14). In summary, the velvet domain of VelB exhibits an IDD, which presence is conserved among filamentous fungi, but the amino acid sequence differs greatly between different genera. This shows that the structural evolution of unstructured domains is much faster than of structured domains.

The velvet domain and the Rel homology domain might have evolved from a common origin. A possible linker between animals and fungi is the unicellular organism Capsaspora owczarzaki, which contains a velvet domain protein as well as a Rel homology domain protein (Ahmed et al., 2013; Sebé-Pedrós et al., 2011). This organism belongs phylogenetically to the Filasterea clade and is, together with the Choanoflagellata, Ichithyospreans and Corallochytreans, one of the closest relatives to animals (FIGURE 48).

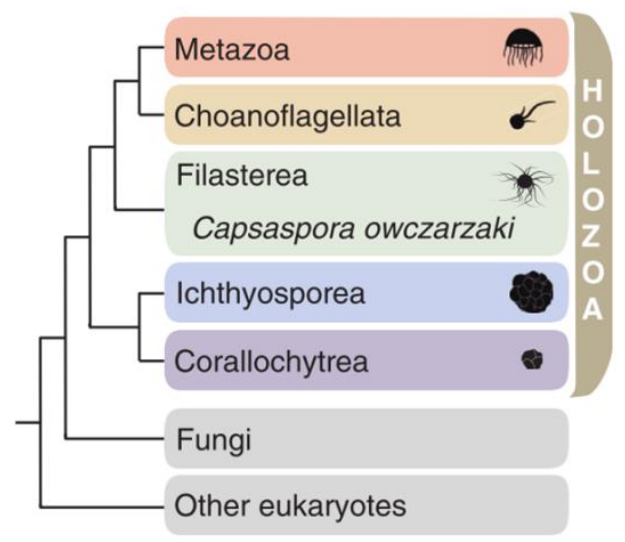

FIGURE 48: Phylogenetic position of Capsaspora owczarzaki.

The schematic phylogenetic tree depicts the position of $C$. owczarzaki as a member of the Filasterea clade, which is one of the closest relatives to animals (modified from Ferrer-Bonet and Ruiz-Trillo, 2017). 
In silico analyses were conducted to examine whether the Rel homology domain of one of the human NF-kB protein family members (RelA/p65, RelB, c-Rel, NF-kB1/p50 and NF-kB2/p52) or the velvet or Rel homology domain of one of the mentioned proteins of $C$. owczarzaki is also interrupted by a domain of intrinsically disorder. Therefore, disorder predictions were carried out employing the DISOPRED program (Ward et al., 2004a). The diagram (FIGURE 49) depicts the intrinsic disorder prediction where the $x$-axis describes the amino acid positions within the protein sequence and the $y$-axis represents the confidence score. This score, which cut-off was set to 0.5 , presents the probability of an amino acid to be disordered within the analyzed protein sequence. The higher the score, the more likely is the disorder. A. nidulans VelB harbors the velvet domain from amino acid residue 53 to 351 . The intrinsic disorder prediction shows, that this domain is interrupted by the intrinsically disordered domain (FIGURE 49, blue arrow). Furthermore the $\mathrm{N}$ - and $\mathrm{C}$-terminus of the VelB protein, outside of the velvet domain, are predicted to be intrinsically disordered.

The predicted velvet domain protein of $C$. owczarzaki spans from amino acid 220 to 407 (according to ScanProsite (de Castro et al., 2006), CDD (Marchler-Bauer et al., 2017) and InterproScan domain predictions (Jones et al., 2014)) and is projected to be a complete structured domain (FIGURE 49, red arrow). The prediction for the RHD protein of $C$. owczarzaki was ambiguous, as different prediction tools vary in the outcome of the projected domains. The $\mathrm{RHD}$ presumably spans from amino acid residues 249 to 515 , which is predicted to be a structured domain harboring few disordered amino acid chains (FIGURE 49, green arrow).

The RHD domains of the five NF-kB protein family members are predicted not to be interrupted but to represent fully structured domains (FIGURE 49, black arrows). This indicates that the $\mathrm{RHD}$ and the velvet domain can share a common ancestor but the VelB intrinsically disordered domain evolved separately and is fungi-specific. 


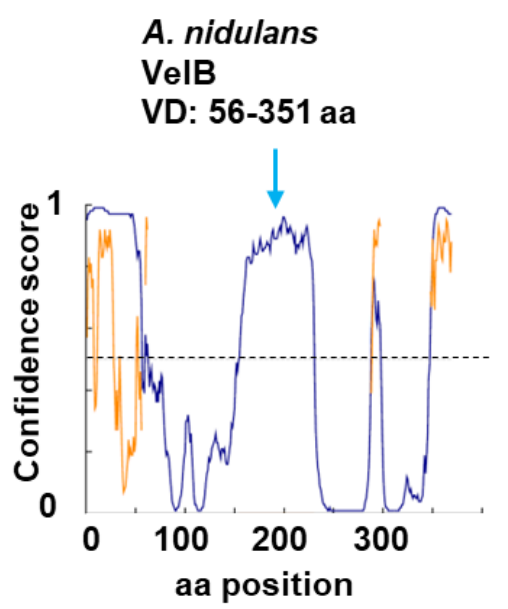

H. sapiens

RelA/p65

RHD: 19-306 aa

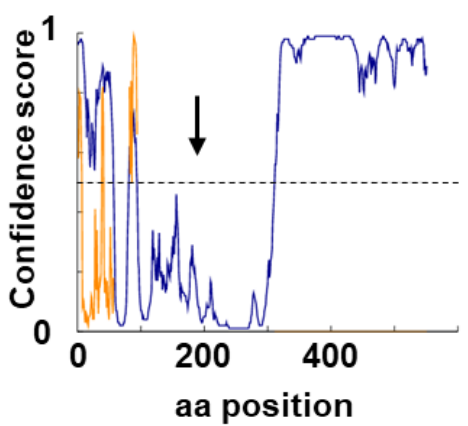

H. sapiens

NF-kB1/p50

RHD: 42-367 aa

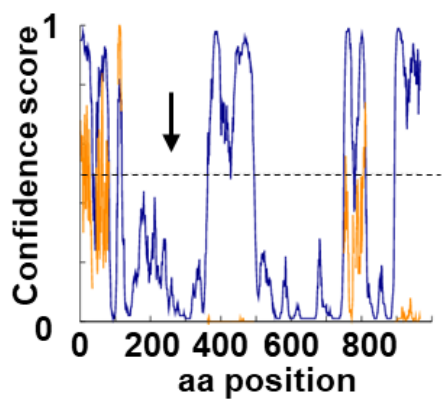

C. owczarzaki

Velvet domain protein

VD: 220-407 aa

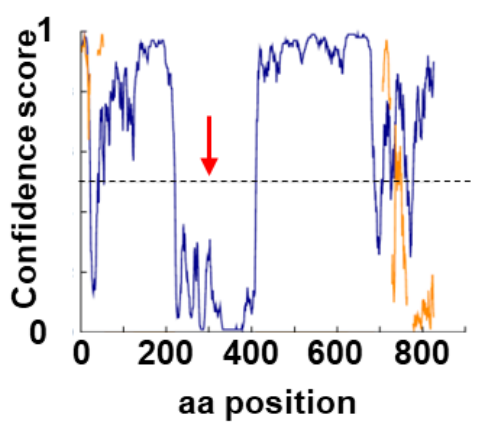

H. sapiens

RelB

RHD: $125-440$ aa

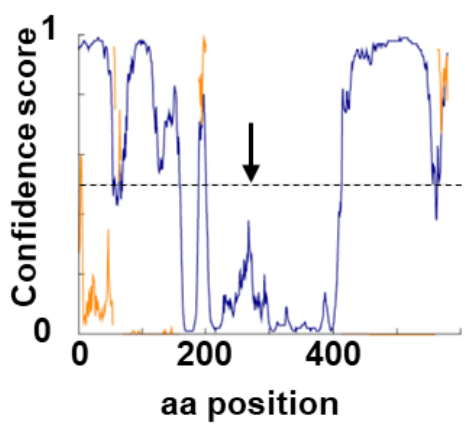

H. sapiens

NF-kB2/p52

RHD: $38-343$ aa

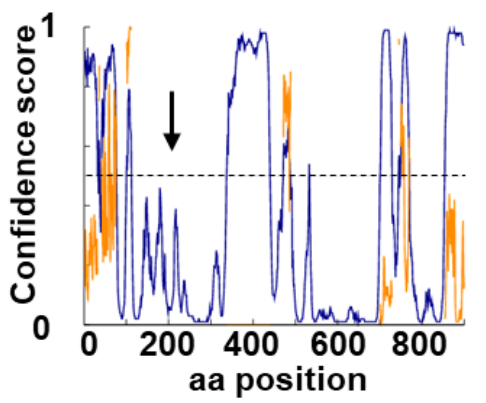

C. owczarzaki

Rel domain protein

RHD: 249-515 aa

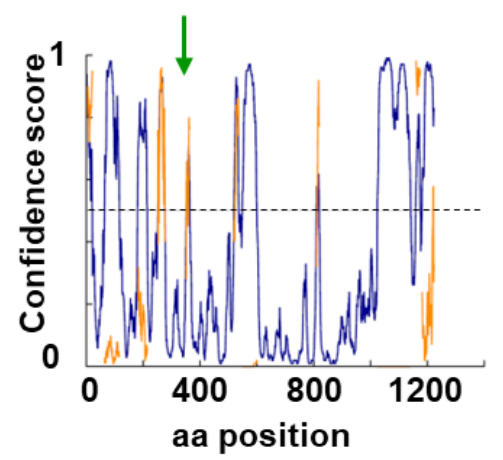

H. sapiens

c-Rel

RHD: 8-294 aa

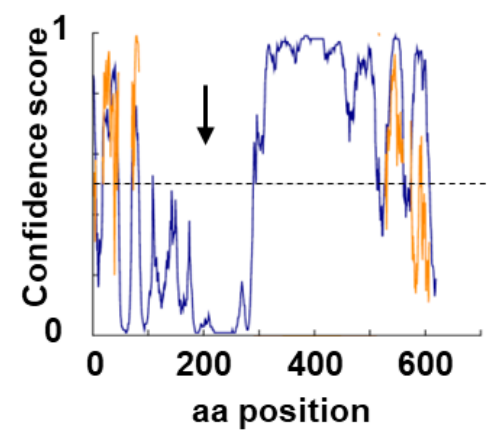

disordered state -

protein binding

FIGURE 49: Intrinsic disorder prediction in comparison to A. nidulans VelB.

Employment of the DISOPRED program (Ward et al., 2004a) revealed structure predictions of C. owczarzaki velvet domain (VD, red arrow = structured) and Rel homology domain (RHD, green arrow $=$ structured) proteins and members of the human RHD containing NF-kB protein family compared to $A$. nidulans VelB. The $A$. nidulans VelB VD is interrupted by an intrinsically disordered domain (blue arrow) but the domains of the other proteins are completely structured (black arrows). The dotted line represents the cut-off of 0.5 of the confidence score. 


\subsection{Disordered domains play central roles as hubs in protein interaction networks}

Proteins comprise two fundamental classes of functional building blocks, structured domains and intrinsically disordered regions (Lee et al., 2015). Hybrid proteins, which contain ordered and unordered protein regions, are characterized by their conformational flexibility and structural plasticity. They play a role in interaction networks as hub proteins, which bind to a variety of interaction partners (Patil et al., 2010). Initially, ID regions were considered to be passive linkers, which only connect the structured domains (Lee et al., 2015). Nowadays, it is well established that they actively contribute to the diverse functions mediated by proteins and in silico studies indicate that more than half of all known eukaryotic proteins contain long regions of disorder (Lee et al., 2015; Uversky, 2015). It is critical to differentiate between long continuous intrinsically disordered regions and short disordered sequences, as these are less likely to be functionally relevant (Ward et al., 2004b). The flexibility of proteins with disordered regions allows the interaction with diverse partners, which expands for example their potential repertoire to respond to environmental changes and signals (Dyson, 2016). The functions of ID regions within several proteins have been identified experimentally and include the recognition of DNA and proteins, the regulation of transcription and translation and targeted protein degradation (Lobley et al., 2007). The human tumor protein p53 is for example a prominent hub protein, which interacts with a variety of other proteins and is a key player in a broad signaling network, including the expression of specific genes involved in processes such as cell cycle progression, apoptosis induction, DNA repair and the response to cellular stress (Oldfield et al., 2008). p53 is referred to as the "guardian of the genome" as it regulates the expression of genes, which govern the major defenses against tumor growth (Kang and Rosenwaks, 2018).

The 99 residues of the VelB IDD might regulate the function of VelB and its binding to different interaction partners in different ways: (1) the conformational state of the protein can be changed, (2) posttranslational modifications (PTMs) can alter and extend the chemical

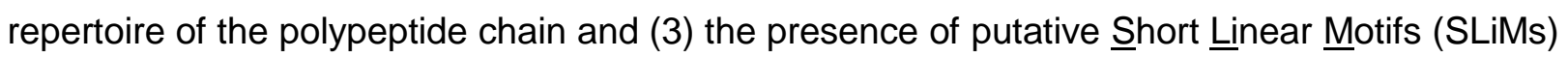
can be involved in recognition and targeting activities with other proteins (Babu et al., 2012; Buljan et al., 2013; Gsponer and Babu, 2009; Mao et al., 2013). This will be discussed in the following chapters.

\subsubsection{VelB as hub in interaction networks}

32 putative interaction partners were exclusively identified in the GFP pull downs for VelB ${ }^{I D D} \Delta_{-}$GFP (TABLE 7). These proteins were grouped according to their putative function or subcellular localization in following categories: translation, primary metabolism, RNA 
maturation and processing, membrane/cell wall, signaling, cell compartments, DNA binding and unknown function. 14 of these proteins are predicted to have a nuclear localization signal with high probability (Kosugi et al., 2009). VelB is a hybrid protein, which consists of the structured velvet domain, which is interrupted by a long intrinsically disordered domain of 99 amino acids. This ensures VelB certain conformational flexibility and structural plasticity, which might assign VelB the role as a hub protein in interaction networks. VelB might exist in a conformational state, which "mimics" the absence of the intrinsically disordered domain. A change in the conformational state of VelB might be triggered by specific environmental circumstances, such as illumination, nutrient supply, $\mathrm{pH}$, temperature or other stressors. This might enable one of the proteins from the GFP pull down to bind to VelB (outside the IDD). This protein could also enhance the nuclear entry of VelB and influence selective heterodimer formation. Possible interaction partners of this suggested "specific conformational state" are discussed below.

The list of putative interaction partners contains several attractive candidates. For example, two NACHT domain containing proteins were identified (AN7222, AN5168), one with an additional Ankyrin repeat domain (AN5168). NACHT domains are named after proteins containing them (NAIP (NLP family apoptosis inhibitor protein), $\underline{\text { CIITA (} 2 T A ~ o r ~ M H C ~ c l a s s ~} \underline{I}$ transcription activator), HET-E (incompatibility locus protein from Podospora anserina) and IEP1 (TP1 or telomerase-associated protein 1)). They are one kind of domains of NOD (nucleotide oligomerization domain)-like receptor (NLR) nucleotide-binding domains of plants and animals (Uehling et al., 2017). NLRs are intracellular receptors, which contribute to the recognition of certain environmental signals and initiate the appropriate response in plants and animals. In contrast, not much is known how fungi interact with their environment, including interactions with viruses, bacteria, protists, amoeba, plants and animals (Uehling et al., 2017). A repertoire of up to $200 \mathrm{NLRs}$ is encoded in the available fungal genomes, which is highly variable and shows intraspecific diversity (Dyrka et al., 2014). Beside the NACHT domain as nucleotide-binding domain, NLRs comprise variable protein-protein interaction domains. The NACHT domain protein identified here contains an ankyrin repeat domain (AN5168) or a tetratricopeptide-like helical domain (TPR) (AN7222) as protein-protein interaction domains. It is suggested that fungal NLRs operate in multimeric complexes and recruit downstream activators of corresponding pathways (Uehling et al., 2017). The elucidation of fungal communication based on NLRs would be a possibility how fungi are able to identify and respond to their biotic environment. VelB could be a downstream factor of one of these $\mathrm{NACHT}$ domain proteins under certain conditions, initiating the accurate response on the transcriptional level. This could require the masking or cleaving of the VeIB IDD to achieve the "correct" conformational state. 
Two putative chromatin remodeling factors (CRF), which are orthologous to the predicted CRFs of $N$. crassa (orthologs of CRFs of $S$. pombe), are possible interaction partners of VelB and as a complex they might influence gene expression on a transcriptional level. CRFs are usually found in large complexes being involved in chromatin remodeling, DNA repair and activation of transcription (Borkovich et al., 2004). AN2278 is the putative ortholog of the SNF2-family ATP dependent chromatin-remodeling factor Cfr3-1 of $N$. crassa (Snf21 in S. pombe) and AN4187 is the putative ortholog of the TBP associated factor Cfr8-1, (Mot1 in S. pombe) (Borkovich et al., 2004). The Snf21-like Cfr3-1 protein is a highly conserved DNAdependent bromodomain ATPase, which is required for chromatin remodeling in the Swi/Snf complex (Mao et al., 2006; Martens and Winston, 2003). The Swi/Snf complex has been shown to be required for hyphal development in Candida albicans (Mao et al., 2006), which makes it also an attractive interaction partner for VelB, which is a regulator of development as well. Mot1-like proteins can be involved in either gene activation or repression presumably by chromatin remodeling during transcription pre-initiation (Borkovich et al., 2004).

The psi-factor producing oxygenase PpoA was also identified in GFP pull downs of $V_{\text {elB }}{ }^{\mathrm{IDD}} \Delta_{-}$GFP. The expression of certain ppo genes determines the ratio and timing of asexual and sexual development (Tsitsigiannis et al., 2005). VeA regulates the ppoA expression, whereas $\mathrm{PpoA}$ and $\mathrm{VeA}$ both negatively regulate the expression of the asexual master regulator encoding gene brlA (Kato et al., 2003; Tsitsigiannis et al., 2004). PpoA in turn is required for the biosynthesis of the psi-factors $\mathrm{psiBa}$ and $\mathrm{psiC \alpha}$, which enhance sexual development in A. nidulans. It is possible that PpoA forms a complex with VeA and VelB (in the "specific conformational state"), which supports sexual and represses asexual development.

Different proteins involved in signaling pathways, such as the MAP kinase kinase MkkB, the putative phosphotransfer protein PhkA and the predicted p-21 activated kinase family protein Cla4 were identified as well. Also putative guanine exchange factors (AN2130 and AN0463) and a putative GTPase-activating protein (AN7576) were found, which might play a role in regulating the activity of small guanine nucleotide-binding $(G)$ proteins by perceiving extracellular signals and thereby controlling cellular events in time and space (Bos et al., 2007). VelB might be involved in these signaling pathways, influencing the developmental programs and interconnected secondary metabolism. Taken together, the GFP pull down experiments identified interesting candidates, which could involve appropriately folded or modified VelB.

\subsubsection{The regulation of the conformational state of intrinsically disordered proteins}

Transcription factors are rich in intrinsically disordered regions in comparison with other proteins and flank the DNA-binding domain $\mathrm{N}$ - or C-terminally. These regions are besides their 
role in the interaction with other proteins, suggested to have a indirect functional role in the binding of DNA and can also directly interact with DNA (Guo et al., 2012; Staby et al., 2017). The velvet domain proteins are fungal-specific transcription factors and the VelB IDD could be involved in the binding of DNA (Ahmed et al., 2013). Structural analysis revealed that the orientation of VelB and VosA to each other in the VelB-VosA heterodimer is different than the orientation of VosA to itself in the VosA-VosA homodimer (Ahmed et al., 2013). The relative orientation of the individual monomers differs significantly in these complexes. The interaction of both VosA subunits to specific target genes simultaneously requires a kink in the DNA in the VosA heterodimer, whereas for the VelB-VosA heterodimer less DNA bending is needed to get a tight VelB-VosA-DNA complex formation (Ahmed et al., 2013). It is suggested, that the VelBVosA heterodimer binds to different promoter regions compared to the VosA homodimer and regulates transcription of specific target genes (Ahmed et al., 2013). However, it must be considered that the recombinant VosA protein used for crystallization comprises only the $\mathrm{N}$-terminal part of VosA (harboring the velvet domain) from amino acid residue 1 to 190 . The C-terminal part is therefore not present in the crystal structure, which could affect dimer formation of these proteins in vivo. The VelB IDD was presumably cut out during protease treatment for crystallization and is not present in the crystal structure (FIGURE 6). However, different mechanisms of the VeIB IDD being involved in modulating the structure of VelB for tight DNA-binding or a direct interaction between the VeIB IDD and DNA are conceivable as given in following examples.

Disordered regions can adjust various essential features in protein-DNA affinity and for example disorder-to-order transitions can enhance the specificity of DNA binding (Vuzman and Levy, 2012). It was already shown in 1990 that the disordered basic regions of several bZIP. DNA-binding domains form $\alpha$-helices upon binding to DNA (Patel et al., 1990; Staby et al., 2017). Intrinsically disordered regions of transcription factors can be required for both, nonspecific and specific DNA-binding (Fuxreiter et al., 2011). Internal IDs support the appropriate orientation of the DNA reading heads by protein-protein interactions once the cognate sequence is detected and flexible loops or ID sequences can also directly promote the specificity of protein-DNA interactions by direct interaction with the DNA (Garviea and Wolberger, 2001). The POU domain of the Brn-5- transcription factor protein is for example separated by an ID linker into two DNA-binding subdomains, POU $\mathrm{HD}_{\mathrm{D}}$ and $\mathrm{POS}_{\mathrm{s}}$. The crystal structure of Brn-5 bound to the $C R H$ gene promoter revealed that this ID linker inserts into a narrow region of the minor groove of the $C R H$ binding site (Pereira and Kim, 2009; Rohs et al., 2010). All in all, the VelB IDD can have a potential role regarding the specificity or affinity of DNA-binding, thereby modulating the expression of certain target genes. 


\subsubsection{Putative short linear motifs and phosphorylation sites of the A. nidulans VeIB IDD}

The presence of short linear motifs (SLiMs) is another possibility how the VelB IDD confers to the function of VelB regarding the interaction with other proteins or protein stability. Employment of the ELM search revealed 14 putative SLiMs in the VeIB IDD (TABLE 10). The conserved MotifIDD contains one of the predicted SLiM sequences "AMSSS" (FIGURE 37, in turquoise box), which might be a target for ubiquitination by Cullin3 E3-RING ligases (ELM name: DEG_SPOP_SBC_1) and for deubiquitination by ubiquitin hydrolases (DOC_USP7_MATH_1). Furthermore, the VeIB IDD harbors several putative phosphorylation motifs, like the CK1, the NEK2 or the GSK3 phosphorylation recognition site for respective kinases, which partially agree with the DISOPHOS predictions (FIGURE 38). The potential phosphorylated residues S145 or S146 are part of the "SISTAMSS" sequence (highlighted in red) and are therefore potential targets for the phosphorylation by NEK2 or GSK3 kinases. Besides the identification of linear motifs with a potential role in posttranslational modification (PTM), motifs involved in protein-protein interaction were identified. The LIG_SH3_1 motif pattern was detected for the "YPPPPHP" sequence (FIGURE 37, green arrow), which is enriched in proline residues and a possible target for SH3 domain proteins (CHAPTER 3.5.2).

Although a cut-off of 0.01 was chosen for the probability score, one has to consider that there are false negative motif patterns with a high probability score, which still can be existing motifs. One example is the DOC_WW_Pin1_4 motif, which is a recognition site for WW domain containing proteins. This motif has a pattern probability of 0.01543 because the "...[ST]P." pattern is likely to occur in any protein just by random chance. However, there are almost 100 instances listed in the ELM database for the DOC_WW_Pin1_4 motif, which are experimentally confirmed. Another example for a possible false negative prediction (ELM score 0.04678) is the "SATSPSI" sequence (LIG_WD40_WDR4_VDV_2 pattern), which is a variant of the fungi-specific WR5-binding domain involved in histone modification complex assembly. This motif plays a key role in H3K4 methylation and H4K16 methylation. However, it might play a role in the context of the epigenetic control of target genes mediated by velvet methyltransferase complexes. The velvet protein $\mathrm{VeA}$ is for example known to bind to several methyltransferases involved in histone modification (Sarikaya-Bayram et al., 2014). VelB-VeA is associated with the LaeA methyltransferase in the VelB-VeA-LaeA complex and VeA is the scaffolding protein in this complex. Although deletion of laeA in several Aspergillus species demonstrated that LaeA is responsible for regulation of approximately $50 \%$ of secondary metabolite cluster genes, it could not be shown that LaeA is directly linked to histone modification resulting in epigenetic control (Sarikaya-Bayram et al., 2015). However, the possibility of this fungi-specific WR5-binding domain to be a part of the VeIB IDD could render the protein as scaffold protein for the assembly of the respective core histone methylation and 
acetylation complex. It is possible that VelB is also directly part of histone modification complexes and thereby contributes to the control of developmental programs and secondary metabolism.

The VeIB IDD is enriched in serine and tyrosine residues (FIGURE 37), which might be targets for phosphorylation and in silico analyses revealed potential phosphosites in the VelB IDD. This might be similar to the hub protein p53, which contains multiple different posttranslational modifications, such as phosphorylation, acetylation, sumoylation and ubiquitination (Oldfield et al., 2008). Phosphorylation of p53 occurs for example by activated cell cycle check point kinases Chk1 and Chk2. Chk1 phosphorylates several sites within the intrinsically disordered C-terminus of $\mathrm{p} 53$, whereas the $\mathrm{N}$-terminal disordered region is primarily phosphorylated by Chk2 (Shieh et al., 2000). P53 is stabilized by Chk2-induced phosphorylation at Ser20 and supports the accumulation of activated p53 in the nucleus, which binds to several genes, encoding proteins from apoptotic pathways (Bálint and Vousden, 2001; Chen et al., 2005). In conclusion, the VeIB IDD is predicted to possess several SLiMs and potential phoshosites, which might for example make the VeIB IDD a target for degradation.

\subsection{The VelB protein stability is affected by the intrinsically disordered domain}

The precise control of protein biosynthesis and turnover is an essential mechanism to sustain the cellular homeostasis. Protein half-lives impact various cellular processes, such as DNA repair, apoptosis, cell survival, cell differentiation, development and immunity (van der Lee et al., 2014). Alterations in protein half-lives can result in abnormal development and diseases (Ciechanover, 2012). The control of the relative amount of transcription factors is essential to ensure the regulation of specific target genes in response to the changing environment. Transcription factors mostly bind DNA as dimers and abundance of dimers might be assumed as a function of monomer synthesis and degradation (Potoyan et al., 2017; Tsui et al., 2015). The control of VelB degradation is one mechanism for the restriction of cellular VelB and its distribution towards complex formation with VeA to induce sexual development or with VosA or itself to presumably promote asexual development or support spore survival, respectively. VelB possesses an internal intrinsically disordered domain and it has been shown for yeast, mouse and human proteins, that long $\mathrm{N}$-terminal ( $\geq 30$ residues) or internal ( $\geq 40$ residues) intrinsically disordered regions contribute to short protein half-live in vivo (van der Lee et al., 2014). Therefore, the relative protein amount during different developmental stages and the protein stability in cycloheximide assays of the full length VelB-GFP compared to the VelB-GFP lacking the IDD (VelB $\left.{ }^{\mathrm{IDD}} \Delta_{-} \mathrm{GFP}\right)$ was investigated in $A$. nidulans. Whereas the relative amount of VelB-GFP decreases during ongoing development and is barely detectable 
after 12 hours, the VelB ${ }^{\mathrm{IDD}}{ }_{\text {-GFP }}$ fusion protein can still be observed after 48 hours of sexual or asexual development (FIGURE 30). The inhibition of translation with cycloheximide revealed that VelB-GFP is destabilized by the VeIB IDD and in its absence the stability of the VelB protein is increased significantly (FIGURE 31). This suggests that the cell controls the relative amount of VelB by using the IDD as putative target for proteasomal degradation.

The half-life of proteins with intrinsically disordered regions can be influenced either by the presence of short peptide motifs serving as destruction signal or by direct initiation of degradation by the proteasome. ELM search revealed that the VelB IDD contains an $\mathrm{N}$-terminal S/T rich motif ("AMSSS"), which is known as the SPOP-binding consensus (SBC) of the MATH-BTB protein and is usually present in substrates that undergo SPOP/Cul3dependent ubiquitination. The MATH-BTB protein, called SPOP, is part of a multiprotein E3 ligase, in which the MATH domain serves as substrate receptor. Furthermore, the VelB IDD contains potential phosphosites, which might be recognized by F-box proteins and target VelB to the SCF complex for ubiquitination and degradation by the $26 \mathrm{~S}$ proteasome (CHAPTER 4.4.1).

The direct initiation of proteasomal degradation through unstructured regions is another possible mechanism (van der Lee et al., 2014). The proteasome is assembled in a way that the catalytic residues are buried deep within the proteasome core particle, which can be only reached by a long narrow channel (da Fonseca et al., 2012). An internal intrinsically disordered domain with a length of at least 40 residues can reach this site and initiate the degradation from the middle of the protein (van der Lee et al., 2014). Furthermore, it is proposed that degradation rates are connected to the composition of amino acids in the unstructured regions to initiate degradation: the higher the variation of the amino acid sequence, the better the recognition by the $26 \mathrm{~S}$ proteasome (Fishbain et al., 2015). This might be a possible mechanism in VelB half-life control through the IDD. The length of 99 residues with a biased amino acid sequence fulfills the requirements for initiation of proteasomal degradation, which results in a decreased half-life of the VelB protein.

\subsubsection{Fbx18 is involved in the regulation of VelB protein levels}

Protein degradation is controlled on different levels. The autophagy-lysosome system and the ubiquitin proteasome pathway are the two major processes for protein breakdown (Kwon and Ciechanover, 2017). Cullin-RING ligases are the largest known class of ubiquitin ligases (CRL) (Petroski and Deshaies, 2005). The activity of the CRL is mainly controlled by the COP9 signalosome (CSN) (Schwechheimer et al., 2001; Suzuki et al., 2002). The CSN removes covalently attached Nedd8 from CRLs by a deneddylation process and thereby inactivates the E3 ligase. Subsequent binding of CandA disassembles the substrate-adaptor-complex from the cullin core allowing new cycles of substrate binding, which ensures the modularity of CRLs 
(Helmstaedt et al., 2011; Schwechheimer et al., 2001). Thereby, ubiquitin labelling of a plethora of proteins is granted, resulting in their degradation by the $26 \mathrm{~S}$ proteasome. This shows that a tight control of protein degradation is fundamental for the maintenance of the cellular protein equilibrium. The substrate-specificity is determined by the F-box proteins within the SCF complex, one of the best characterized CRLs (Skaar et al., 2013). The substrate recruitment by the F-box proteins represents the ultimate regulation of the activity of particular SCF complexes (FIGURE 50). The rapid and specific attachment of F-box proteins to their targets in response to specific internal or external environmental stimuli, such as stress or starvation, is required to recruit them to the SCF complex to label them for degradation by the 26S proteasome. The interface between F-box proteins and the substrate and the F-box proteins themselves are tightly regulated by diverse mechanisms, such as by restricting the degron access, covalent and non-covalent modification of degrons, regulation of F-box localization and stability (FIGURE 50) (Skaar et al., 2013).

The best characterized F-box proteins bind to short defined and mostly phosphorylated degradation motifs, so called (phospho-) degrons. The use of accessory proteins as cofactors is another mechanism for substrate recruitment. The access to the degron can also be restricted physically and only after a conformational change, achieved for example by phosphorylation, the F-box protein is able to bind. Other covalent modifications despite phosphorylation, non-covalent modifications or dephosphorylation can render the substrates accessible for F-box proteins.

The present study shows that VeIB is destabilized by the IDD. It was further examined whether the cellular amount of VelB is influenced by the absence of F-box proteins in A. nidulans. The availability of a monoclonal antibody against ten residues in the VeIB IDD $\left(\alpha-\right.$ VeIB $^{I D D}=$ aa 152 161) enabled the screening of the relative amount of VelB in 72 fbox deletion mutants of A. nidulans during vegetative growth by western hybridization experiments. Fbox18 was chosen after this pre-screen as candidate with the strongest impact on VelB stability. 


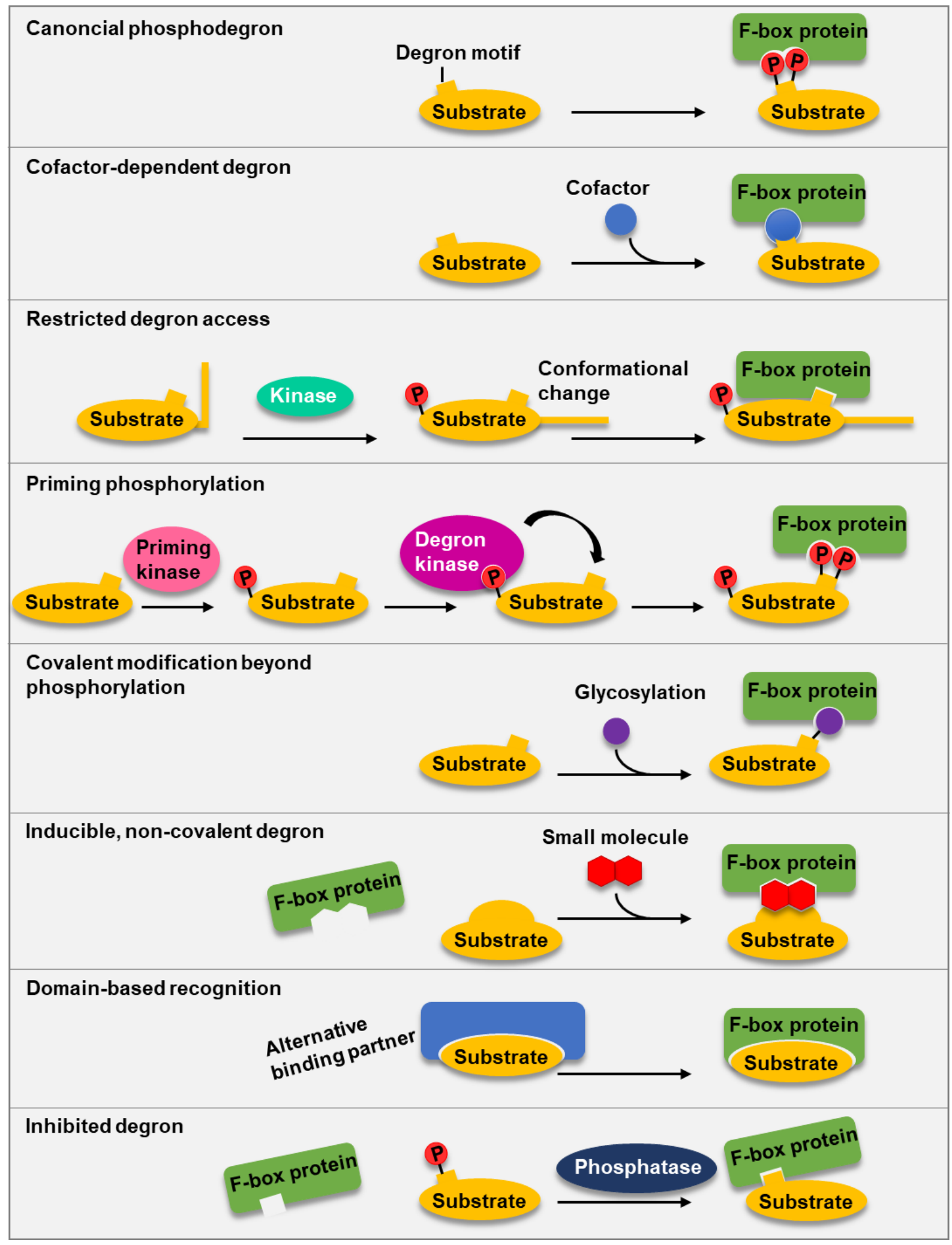

FIGURE 50: Substrate recruitment mechanisms of F-box proteins.

Substrates are recognized by F-box proteins in multiple ways. The regulation of substrate degradation is tightly controlled, for example by combining the different recognition mechanisms. Eight different ways for the substrate recruitment are depicted (adapted from Skaar et al., 2013). 
Relative amount of VelB-GFP decreased to $50 \%$ in the wildtype background when translationa was inhibited with cycloheximide, whereas the protein remains stable in the $f b \times 18$ deletion background. This indicates that Fbx18 either might be the substrate receptor for VelB within the SCF complex, which specifically binds the VeIB IDD or parts of its sequence or label VelB for degradation by the $26 \mathrm{~S}$ proteasome or Fbox18 indirectly reduces the cellular VelB protein levels in A. nidulans. Fbox18 could recognize the VeIB IDD through different mechanisms as depicted in FIGURE 50. The covalent attachment of a cofactor or a posttranslational modification could render the VelB IDD to be directly detected by Fbox18. Another mechanism might be that the IDD has to be modified to enable a conformational change of the VelB protein, which in turn makes it discernible for Fbox18. Furthermore, masking of VelB at the intrinsically disordered domain by an alternative binding partner could prevent substrate recognition by Fbox18. Unmasking of the IDD under specific conditions would make VelB accessible to be targeted by Fbox18 to the SCF complex to label it for proteasomal degradation. However, an indirect control of VelB stability by Fbox18 cannot be excluded.

Velvet proteins have been shown before to be substrates for F-box proteins. FWD1, besides targeting FRQ1 for proteasomal degradation, is also required for the degradation of the $\mathrm{VeA}$ ortholog VE-1 in $N$. crassa, as the absence of FWD-1 leads to an accumulation of VE-1 when translation is inhibited (L.M. Corrachano, p.c. $29^{\text {th }}$ Fungal Genetics Conference). FWD1 of $N$. crassa is the ortholog of Fbox23 in A. nidulans. Deletion of fbx23 in A. nidulans results in constitutive sexual development (von Zeska Kress et al., 2012). The same phenotype was observed in an $A$. nidulans veA overexpression mutant (Bayram et al., 2008). This suggests that Fbox23 of $A$. nidulans might be the substrate receptor for VeA to target it for degradation. The absence of $f b x 23$ presumably results in increased cellular VeA amounts, increasing sexual development.

VelB is a multifunctional developmental regulator and it was shown that its overexpression increases the amount of conidiospores about two-fold (Park et al., 2012b). Therefore, asexual development of the fbox $18 \Delta$ mutant strain was compared to the wildtype. Strains were either point inoculated or the spores distributed over the whole plate and incubated for three and seven days in light, promoting asexual development. The phenotypes do not differ significantly at first glance (FIGURE 36). The $f b \times 18$ deletion mutant is increased in cellular amounts of VelB during vegetative growth. It is possible that during asexual growth conditions other F-box protein/s serve as substrate adaptor for VelB. On the other hand, increased amounts of full length VelB do not have strong effects on $A$. nidulans growth. Quantification of conidiospores and investigation of cellular amounts of VelB in the fbx18D background during asexual development could provide more insight. 


\subsection{The VelB IDD is required for accurate conidiation and secondary metabolite production in A. nidulans}

The formation of specific velvet domain protein dimers is required for the regulation of appropriate developmental programs and interconnected secondary metabolism in response to environmental changes, such as illumination or the availability of oxygen. In the absence of the VelB IDD the control of an accurate ratio of certain dimers is disturbed, as complex formation with VosA or VelB homodimer formation is abolished. Therefore the development and the biosynthesis of secondary metabolites, such as sterigmatocystin, of a vel$B^{I D D}$ deletion strain ( $v e l B^{I D D \Delta}$ ) were compared to the wildtype and the deletion strain completely missing the gene encoding for VelB (velBA). Due to the lack of VelB, the VelB-VeA heterodimer cannot be formed in the velB deletion strain and even in the dark under limiting oxygen supply, promoting sexual development, the formation of sexual fruiting bodies is abolished. The velB ${ }^{I D D_{\Delta}}$ mutant strain is capable of dimerizing with VeA. Sexual development is not impaired and black mature cleistothecia are formed after seven days like in the wildtype strain (FIGURE 23). Although VelB-VosA heterodimer formation is abolished in the vel$B^{I D D_{\Delta}}$ mutant strains, the spore viability seems not to be decreased (FIGURE 24). This indicates that when this dimer cannot be formed, another velvet domain protein dimer, such as VelB-VeA could take over and activate the genes required for the maintenance of viable conidiospores. Examination of spore viability in the $v e / B^{I D D_{\Delta}} / v e A \Delta$ deletion strain, which neither is able to form the VelB-VosA nor the VelB-VeA heterodimer, could provide more insights in future investigations.

\subsubsection{Dehydroaustinol production requires the VelB-VosA heterodimer}

Illuminating conditions and oxygen supply favor asexual development. Conidiophore formation is altered in the $v e l B^{I D D}$ deletion mutant in dependency of the way with which spores were inoculated on the agar plate. Spores were inoculated as a single spot, resulting in radial growing colony from the middle of the plate, or a high spore concentration was used as starting inoculum and spread over the whole plate. The strain lacking the VeIB IDD produces air-borne conidiospores like the wildtype, when grown from a single spot (FIGURE 21). The growth is a little delayed after three days but after seven days it totally resembles the wildtype. This suggests that the IDD and thereby the VelB-VosA heterodimer and presumably the VelB-VelB homodimer, are dispensable for the early light response resulting in conidiation at low spore densities. The inoculum with high spore densities reduced the amount of conidiospores in the vel $B^{I D D}$ deletion mutant (FIGURE 22). Many aerial hyphae are formed instead and conidiospore formation is visible only at the edges of the agar plate. This indicates that the fungus is impaired in conidiospore formation at high spore concentrations. 
This observation correlates with decreased austinol and dehydroaustinol production in the absence of the VeIB IDD when spores were distributed over the whole plate (FIGURE 26). Dehydroaustinol is one compound, which was shown to be involved in signaling the induction of sporulation (Rodríguez-Urra et al., 2012). U-pstream developmental activators (UDA) are responsible for the initiation of conidiation at early stages. Deletion of any of the UDA genes like fluG or one of the flb factors results in the accumulation of aerial hyphae with almost abolished conidiospore production (Oiartzabal-Arano et al., 2016), comparable to the phenotype of the vel $B^{I D D}$ deletion mutant. Therefore, the UDA mutants are denominated as fluffy mutants. This phenotype could be rescued by the addition of dehydroaustinol and diorcinol (Rodríguez-Urra et al., 2012). Theoretically, deletion of any gene encoding for an enzyme participating in the biosynthesis of dehydroaustinol should result in non-sporulating fluffy mutants. A genome wide analysis of polyketide synthases (PKS) revealed that deletion of the aus $A$ gene, encoding the first enzyme in the dehydroaustinol biosynthetic pathway, results in a mutant producing sporulating colonies (Nielsen et al., 2011; Rodríguez-Urra et al., 2012). In addition to that, diorcinol is produced in some sporulation defective mutants, such as the csnE deletion mutant, which even overproduces this compound (Butnick et al., 1984; Nahlik et al., 2010). This leads to the conclusion that the FluG signal involves a group of metabolites including dehydroaustinol and diorcinol, possibly accompanied by other so far unidentified compounds (Rodríguez-Urra et al., 2012).

Like the $v e l B^{I D D_{\Delta}}$ mutant strain, the velB deletion mutant produces less austinol and dehydroaustinol, as well. Furthermore, conidiospore formation is, but not as much as in the $v e / B^{I D D}$ deletion strain, reduced when $v e / B$ is deleted. This indicates that reduced dehydroaustinol biosynthesis leads to these reductions in conidiospore formation. Both strains produce less austinol and dehydroaustinol and are not capable of the formation of the VelBVosA heterodimer, which therefore seems to be required for the activation of the biosynthesis of these compounds.

\subsubsection{High spore densities require the VelB IDD for conidiospore formation in}

\section{A. nidulans}

The velB and the vel$B^{I D D}$ deletion mutant produce less conidiospores and less dehydroaustinol. In contrast to the velB deletion strain, absence of the VeIB IDD additionally results in the production of many aerial hyphae at high spore densities.

The density-dependent mechanism of quorum sensing (QS) enables microbes to monitor and respond to their population density. QS is a cell-to-cell signaling system and is best described for bacteria, which respond at a certain concentration to chemical hormone-like organic compounds (Reading and Sperandio, 2006). This results in the alteration of gene expression of an entire population, thereby inducing for example biofilm formation and the production of 
virulence factors (Reading and Sperandio, 2006). Quorum sensing is not restricted to prokaryotic species and this system is also well established in fungi (Albuquerque and Casadevall, 2012). The discovery of farnesol in the human pathogen C. albicans was the first identification and isolation of a fungal QS molecule, followed by the identification of tyrosol, phenylethanol and tryptophol (Chen et al., 2004; Hornby et al., 2001). The cellular density determines yeast-to-hypha transition and farnesol is one compound which is responsible to maintain the yeast form at high cell densities. At low cell densities filamentous growth is favored (Albuquerque and Casadevall, 2012; Wongsuk et al., 2016). It has been shown that high cell densities increase conidiation in A. flavus (Brown et al., 2008). A QS mechanism was suggested, which includes a factor(s) for conidiation activation in high-density cultures, whereas a factor(s), produced in low-density conditions, stimulates sclerotial formation and/or inhibits conidiospore formation (Brown et al., 2008). The examination of oxylipin-deficient lipoxygenase and dioxygenase mutants revealed that lipoxygenase-derived oxylipin molecules play a role in the quorum-like morphology shift in $A$. flavus (Brown et al., 2008). The psi-factor producing oxygenase PpoA was identified as putative interaction partner in GFP pull downs with the truncated VelB ${ }^{\mathrm{IDD} \Delta_{-}}$GFP fusion protein (CHAPTER 4.1.2). PpoA is required for the biosynthesis of the psi-factors $p s i B \alpha$ and $p s i C \alpha$, which enhance sexual development in A. nidulans (Tsitsigiannis et al., 2004). A potential involvement of these compounds in a QSderived mechanism cannot be excluded. Under specific conditions certainly folded VelB could interact with PpoA and influence the $A$. nidulans growth at high spore densities. The velB ${ }^{I D D \Delta}$ mutant strain produces significantly less conidiospores and many aerial hyphae at high spore concentrations. For A. flavus it was described, that high spore density activates conidiation. A possible explanation could be that the vel$B^{I D D_{\Delta}}$ mutant strain is presumably not capable to sense the QS molecules and therefore does not react with appropriate conidiospore production.

vel$/ B^{I D D_{\Delta}} /$ velvets double deletion mutants were analyzed to uncover, which velvet dimer proteins might be involved in this putative quorum sensing mechanism (FIGURE 41). The velB $B^{I D D_{\Delta}} / v e A \Delta$ and $v e / B^{I D D_{\Delta}} /$ vosA $\Delta$ mutant strains show similar phenotypes to the respective single velvet deletion strains during asexual development promoting conditions. In contrast, the vel$B^{I D D_{\Delta}} /$ velC $\Delta$ double deletion mutant resembles the vel$B^{I D D}$ single deletion mutant after three days of growth as it also forms many aerial hyphae instead of sporulating conidiophores. Contrary to the vel$B^{I D D_{\Delta}}$ mutant, this phenotype is rescued in the velB $B^{I D D_{\Delta}} / v e / C \Delta$ double deletion mutant after seven days of growth in the light and conidiospores are produced on the whole plate and not only in the edges. When in the absence of the VelB IDD VelB-VosA heterodimer and VelB-VelB homodimer formation are abolished, the ratio of possible velvet domain protein dimers might be shifted to the direction of VelB-VeA, VosA-VosA and VelC-VosA. The $v e l B^{I D D_{\Delta}} / v o s A \Delta$ double mutant resembles the phenotype of the $\operatorname{vos} A$ single deletion mutant, 
which thereby excludes the VelB-VeA heterodimer to be responsible for reduced conidiation at high spore concentrations.

So far, VelC-VosA interaction was only shown in vitro by Park and co-workers. In this study, VelC-GFP pull downs confirmed VelC-VosA heterodimer formation during early sexual development in $A$. nidulans (TABLE 11). Distribution of VosA to interact with itself or with VelC might be assured by several factors. First, VelC-VelC homodimer formation cannot be excluded. Second, velC is mostly expressed during early sexual development and not as abundant as VosA, which is also expressed during asexual development (Park et al., 2014). Third, the instability of the VeIC protein (FIGURE 40) guarantees controlled abundance of VelC and therefore a regulation of VelC-VosA heterodimer formation, which is supported by the presence of the PEST domain. Furthermore, VeIC was found to interact with proteins involved in protein degradation (TABLE 11), such as the putative subunit of the $20 \mathrm{~S}$ core proteasome, the putative ortholog of the A. flavus regulatory subunit Rpt2 (AN2213) and a putative ortholog of the A. fumigatus DppV, a dipeptidyl-peptidase.

Taken together, it is conceivable that absence of the VeIB IDD and thereby abolished VelB-VosA heterodimer and presumably VelB-VelB homodimer formation, impairs a quorum sensing mechanism, which results in decreased conidiospore formation. On the other hand, absence of the VeIB IDD moves the dimer formation ratio towards VelB-VeA, VosA-VosA and VelC-VosA. Examination of the double deletion mutants implies, that the VelC-VosA and VosA-VosA dimers might be involved during different developmental time points in the quorum-like mechanism, decreasing the conidiospore production at high spore densities. Furthermore, the formation of a VelC-VelC homodimer cannot be excluded, which also might contribute to the regulation of conidiospore formation.

\subsubsection{The VelB IDD is required for the accurate regulation of sterigmatocystin production}

VeA forms together with VelB and the methyltransferase LaeA the heterotrimeric velvet complex, representing a key element of the coordinated regulation of secondary metabolism and development (CHAPTER 1.5) (Bayram et al., 2008; Bok and Keller, 2004; Schumacher et al., 2015). Deletion of laeA results in silenced secondary metabolism and its overexpression increases secondary metabolite production. LaeA is a master regulator of secondary metabolism, which is conserved in the fungal kingdom, whereas its role in secondary metabolite regulation is specific in different species (Amaike and Keller, 2009; Bok et al., 2006; Bok and Keller, 2004). LaeA and members of the velvet protein family contribute to the virulence of several pathogenic fungi, presumably by activating mycotoxin production (Estiarte et al., 2016; Kumar et al., 2016; López-Díaz et al., 2017). 
The influence of abolished VelB-VosA and therefore enhanced VelB-VeA heterodimer formation (and velvet complex formation) on sterigmatocystin production was investigated in the velB $B^{I D D_{\Delta}}$ mutant. VelB-VosA indirectly represses the production of sterigmatocystin in the wildtype, as VosA can replace $V e A$ in the VelB-VeA heterodimer after its shuttle into the nucleus. This results in the moderate biosynthesis of sterigmatocystin during wildtype conditions. In the absence of the VeIB IDD the formation of VelB-VeA (and presumably also the VelB-VeA-LaeA complex) is enhanced as VelB-VosA heterodimer formation is abolished. Without the different velvet complex formations in the $v e / B^{I D D_{\Delta}}$ mutant, elevated levels of sterigmatocystin were observed, as its biosynthesis is increased.

Traces of sterigmatocystin are produced in the absence of velB (FIGURE 27), suggesting a residual activity of VeA-LaeA (Bayram et al., 2008). Metabolite extraction from the velB $B^{I D D_{\Delta}} /$ velvets double deletion mutants revealed that the velB ${ }^{I D D_{\Delta}} / v e / C \Delta$ but not the velB $B^{I D D_{\Delta}} / v e A \Delta$ and $v e / B^{I D D_{\Delta}} / \operatorname{vos} A \Delta$ mutant strain produces an increased amount of sterigmatocystin. The $v e l B^{I D D} / v e A \Delta$ does not form the VelB-VeA heterodimer and the VelB-VeA-LaeA velvet complex anymore, which explains abolished sterigmatocystin production. In contrast to the vel$B^{I D D_{\Delta}} /$ vos $A \Delta$ mutant, the vel $B^{I D D_{\Delta}} / v e l C \Delta$ double deletion mutant is still capable of the formation of the VosA-VosA homodimer. In yeast-two-hybrid assays it was shown by Bayram and co-workers that VosA interacts with LaeA in vitro (Bayram et al., 2008). A VosA-LaeA (or VosA-VosA-LaeA) complex also might induce sterigmatocystin production, which is a possible explanation for the overproduction in the vel $B^{I D D_{\Delta}}$ single and $v e / B^{I D D_{\Delta}} / v e / C \Delta$ double deletion mutant and the traces of sterigmatocystin in the velB deletion strain. Altogether, appropriate sterigmatocystin production requires a balanced ratio of velvet domain proteins in $A$. nidulans.

\subsubsection{Velvet domain protein dimers abolish the production of certain secondary metabolites}

Genes encoding secondary metabolite biosynthetic proteins are mostly organized in clusters. These secondary metabolite gene clusters are often silenced under laboratory conditions in wildtype strains and can be activated during fungal development in response to environmental signals or when other organisms are present (Bayram et al., 2016; Gerke et al., 2012). Secondary metabolites include mycotoxins, which serve as defense mechanism against fungivores and competitors (Kempken, 2011). Recently it was shown, that the VelB-VeA-LaeA velvet complex of $A$. nidulans, which induces sterigmatocystin production, is required to compete against $D$. melanogaster (Regulin and Kempken, 2018).

Different approaches, like specific culture conditions (Sanchez et al., 2010) or the deletion of $v e A$ (Bok et al., 2013) lead to the activation of the orsellinic/F-9775A and B cluster, which is silenced under normal conditions. The velB deletion mutant produces, like the veA deletion 
mutant, a brown pigment, which is released into the agar. Metabolite extraction uncovered, that the velBA mutant strain also produces orsellinic acid and F-9775A and B (FIGURE 26). This indicates that the VelB-VeA represses the production of these metabolites in the wildtype background.

Metabolite extraction and examination of thin layer chromatography silica plates at $254 \mathrm{~nm}$ and white light of $v e l B^{I D D}$ and velvet single and double deletion mutants revealed more differences in the secondary metabolite pattern. The vel$/ B^{I D D_{\Delta}}$ mutant is abolished or delayed in the production of certain uncharacterized secondary metabolites (FIGURE 28). Furthermore, the delay of conidiospore formation in the $v e / B^{I D D_{\Delta}} / v e l C \Delta$ mutant strain correlates with the production of a specific secondary metabolite. This secondary metabolite is absent in the $v e l B^{I D D}$ single deletion mutant after three and seven days of growth in the light, but delayed in the velB ${ }^{I D D_{\Delta}} /$ velC $\Delta$ mutant strain, as it is produced after seven days (FIGURE 42). In the wildtype strain this compound could be already detected after three days of growth. This implies that the VosA-VosA homodimer and/or VelC-VosA heterodimer are involved in the regulation of this compound at different developmental stages.

Examination of a velB $\Delta / v e l C \Delta$ double deletion mutant supports that the velvet domain proteins are required for the orchestration of development and secondary metabolism. This strain produces even more conidiospores than the wildtype strain and is still, due to abolished VelB-VeA heterodimer formation, not able to produce sexual fruiting bodies. The VelC-VosA heterodimer is presumably required for the repression of asexual development. In the velB $\Delta /$ velC $\Delta$ mutant strain, this dimer cannot be formed anymore, what results in the increased

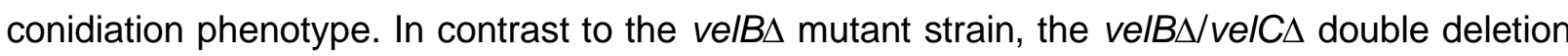
mutant does not only release brown pigments into the agar but the colony is also surrounded by a yellow pigmented ring (FIGURE 44). This leads to the suggestion that one or more secondary metabolite gene clusters, which are silent in the wildtype situation by simultaneous repression by VelB-VeA and VelC-VosA, are expressed in the velB $/$ velC $\Delta$ double mutant when these dimers cannot be formed. VelC is suggested to be expressed and involved at early stages of sexual development (after 24 and 48 hours of growth promoting sexual development, respectively) (Park et al., 2014). The presence of a PEST domain in connection with the results from western hybridization experiments suggests that the VelC protein is instable (FIGURE 40). The selective biosynthesis of VelC and its instability might be a putative mechanism how VelC-VosA heterodimer formation and its regulatory functions are controlled.

The deletion of all four velvet genes results in decreased conidiospore formation and abolished sexual development. Furthermore, the agar is colored in a dark red (FIGURE 46). Taken together, the accurate control of development and secondary metabolite production requires the velvet domain proteins, but they are not essential for $A$. nidulans. 


\subsection{Conclusion and outlook}

Velvet domain proteins are well described to control the regulation of development and interconnected secondary metabolism in filamentous fungi (Bayram and Braus, 2012; Gerke and Braus, 2014). The temporal and spatial distribution of specific velvet domain protein dimers results in adequate reactions in response to the changing environment. The interruption of the velvet domain is conserved in VelB orthologs but amino acid sequences of the IDDs vary considerably, which might be due to the rapid evolution of disordered regions in contrast to structured domains. This study shows that the VeIB IDD might enable a molecular mechanism responsible for the distribution of cellular VelB to form different complexes in A. nidulans.

The VeIB IDD displays a possible target for proteasomal degradation, as it destabilizes the VelB protein (FIGURE 30, FIGURE 31). F-box18 decreases the relative amount of VelB (FIGURE 35) and might be its substrate receptor within the SCF complex to label it for degradation by the $26 \mathrm{~S}$ proteasome. Furthermore, a comparison of VelB with or without IDD revealed a novel control function for velvet domain protein dimer formation. The interaction partners of VelB with IDD differ from the interaction partners of VelB without IDD. The VelB IDD is required for the stable VelB-VosA heterodimer formation in $A$. nidulans and for VelB-VelB homodimer formation in vitro. In contrast, VelB-VeA heterodimer and velvet complex VelB-VeA-LaeA formation are independent of the VelB IDD. This suggests that the IDD supports selective heterodimer formation and provides a specific surface for altered protein-protein interactions, which changes the potential of velvet domains to bind DNA.

In summary, absence of the VeIB IDD shifts the dimer formation towards VelB-VeA and as VelB ${ }^{\mathrm{IDD} \Delta}$ does not interact with VosA, VosA-VosA and presumably VelC-VosA dimer formation might be increased. The altered ratio of velvet domain protein dimer formation is reflected in decreased conidiospore formation at high spore concentrations and altered secondary metabolite production. The biosynthesis of sterigmatocystin is increased and austinol and dehydroaustinol production is decreased in the vel$B^{I D D_{\Delta}}$ mutant strain. A summarizing model in FIGURE 51 depicts the influence of selective heterodimer formation of VelB with the other velvet regulators in $A$. nidulans. 


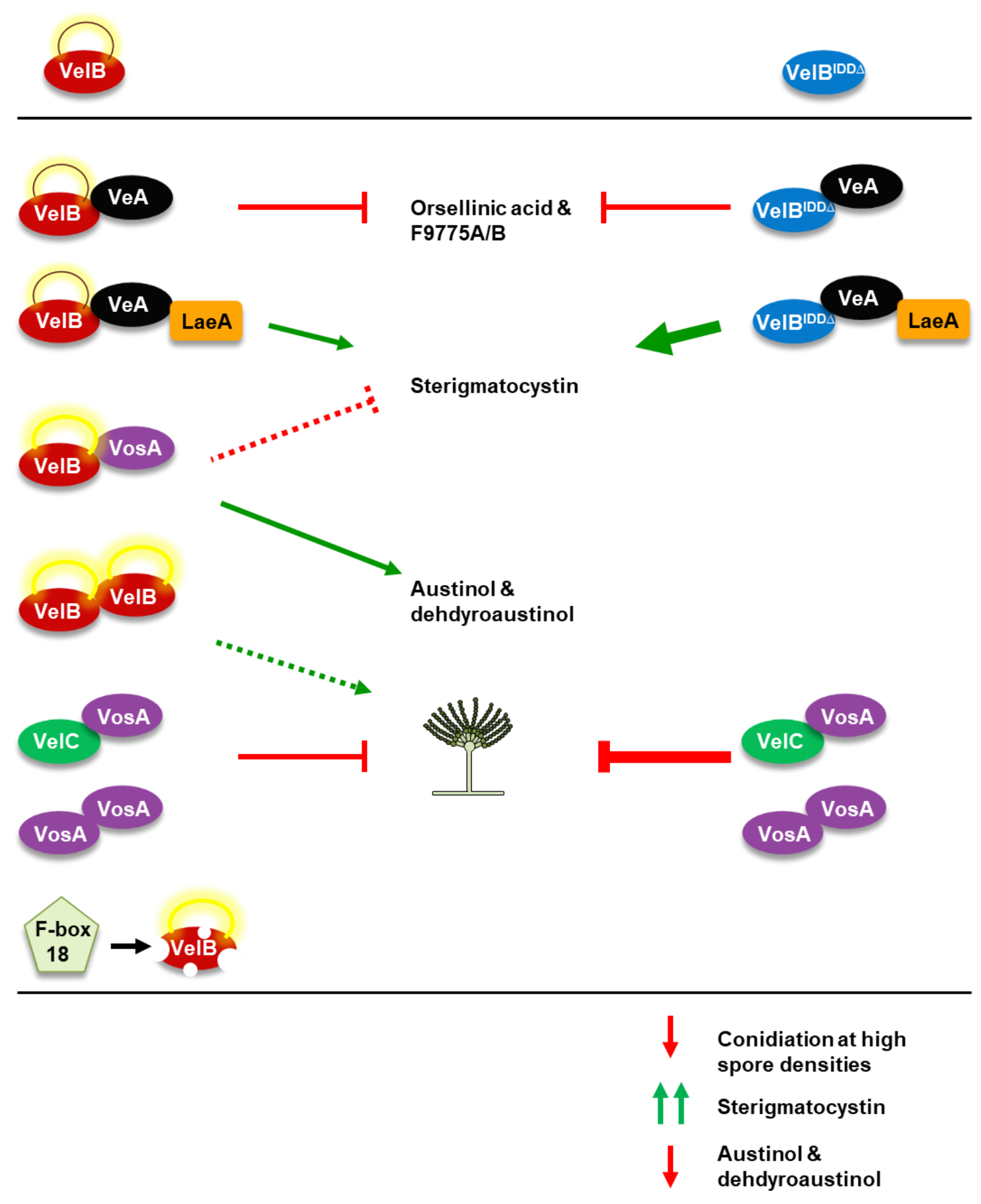

FIGURE 51: Comprehensive model of the consequences of VelB selective dimer formation in A. nidulans.

The model depicts the dimer formation of VelB with other velvet factors in the absence or presence of the VeIB IDD. Green arrows indicate positive and red lines negative influences on secondary metabolite production. The VeIB IDD destabilizes the protein and F-box18 reduces the VelB protein level. Lack of the VeIB IDD results in decreased conidiation (red arrow), increased sterigmatocystin production (two green arrows), and decreased austinol and dehydroaustinol biosynthesis (red arrow). 
The velvet domain shows structural similarities with the Rel homology domain of the mammalian NF-kB transcription factor family (Ahmed et al., 2013). The NF-kB protein family members, like the velvet domain proteins, form specific homo- and heterodimers for the precise regulation of many target genes in response to environmental stimuli. The mechanism, which is needed for the regulation of the abundance and ratio of velvet domain protein homoand heterodimers, was not unraveled so far. The VeIB IDD harbors putative short linear motifs and putative phosphosites. Investigation of strains, which contain mutations of specific amino acid residues of these SLiMs or of putative phosphosites, could be a future perspective to unravel the molecular mechanism, which supports the selective dimer formation of VelB. Furthermore, examination of the structure of the VeIB IDD by nuclear magnetic resonance spectroscopy could provide more information about its function.

The VeIB IDD is required for conidiospore formation at high spore densities. A better understanding of an underlying putative quorum sensing-derived mechanism and presumptive involved metabolites, such as oxylipins, could broaden our knowledge about communication mechanisms of $A$. nidulans. This acquired knowledge could be transferred to pathogenic fungi to investigate possibilities to prevent infections and their deleterious effects on humankind. Absence of the VelB IDD moves the velvet domain protein ratio towards complex formation with VeA. This results in a drastically increased biosynthesis of the carcinogenic mycotoxin sterigmatocystin. It is important to understand how this mechanism works to prevent VelB-VeA dimer formation and subsequent sterigmatocystin production. An extended knowledge of appropriate approaches against the contamination of food and deleterious infections on humankind by fungi is another required target of future studies.

Taken together, this study provides insights into a novel control mechanism for selective dimer formation in connection with the execution of appropriate developmental programs and the biosynthesis of certain secondary metabolites. 


\section{Literature}

Adams, T.H., Boylan, M.T. and Timberlake, W.E. (1988). brlA is necessary and sufficient to direct conidiophore development in Aspergillus nidulans. Cell $\underline{54}, 353-362$.

Adams, T.H., Deising, H. and Timberlake, W.E. (1990). brlA requires both zinc fingers to induce development. Mol. Cell. Biol. 10, 1815-1817.

Adams, T.H., Wieser, J.K. and Yu, J.H. (1998). Asexual sporulation in Aspergillus nidulans. Microbiol. Mol. Biol. Rev. $\underline{62}, 35-54$.

Ahmed, Y.L., Gerke, J., Park, H.S., Bayram, Ö., Neumann, P., Ni, M., Dickmanns, A., Kim, S.C., Yu, J.H., Braus, G.H. and Ficner, R. (2013). The velvet family of fungal regulators contains a DNA-binding domain structurally similar to NF-kB. PLoS Biol. 11 , e1001750. doi:10.1371/journal.pbio.1001750.

Alberti, F., Foster, G.D. and Bailey, A.M. (2017). Natural products from filamentous fungi and production by heterologous expression. Appl. Microbiol. Biotechnol. 101, 493-500.

Albuquerque, P. and Casadevall, A. (2012). Quorum sensing in fungi - a review. Med. Mycol. 50, 337-345.

Altschul, S.F., Gish, W., Miller, W., Myers, E.W. and Lipman, D.J. (1990). Basic local alignment search tool. J. Mol. Biol. 215, 403-410.

Amaike, S. and Keller, N.P. (2009). Distinct roles for VeA and LaeA in development and pathogenesis of Aspergillus flavus. Eukaryot. Cell $\underline{8}, 1051-1060$.

Ameyar, M., Wisniewska, M. and Weitzman, J.B. (2003). A role for AP-1 in apoptosis: The case for and against. Biochimie $\underline{85}, 747-752$.

Andrianopoulos, A. and Timberlake, W.E. (1994). The Aspergillus nidulans abaA gene encodes a transcriptional activator that acts as a genetic switch to control development. EMBO J. 14, 2503-2515.

Ashida, H., Kim, M. and Sasakawa, C. (2014). Exploitation of the host ubiquitin system by human bacterial pathogens. Nat. Rev. Microbiol. 12, 399-413.

Axelrod, D., Gealt, M. and Pastushok, M. (1973). Gene control of developmental competence in Aspergillus nidulans. Dev. Biol. 34, 9-15.

Babu, M.M., Kriwacki, R.W. and Pappu, R.V. (2012). Versatility from protein disorder. Science 337, 1460-1461.

Babu, M.M., Luscombe, N.M., Aravind, L., Gerstein, M. and Teichmann, S.A. (2004). Structure and evolution of transcriptional regulatory networks. Curr. Opin. Struct. Biol. 14, 283291.

Bai, C., Sen, P., Hofmann, K., Ma, L., Goebl, M., Harper, J.W. and Elledge, S.J. (1996). SKP1 connects cell cycle regulators to the ubiquitin proteolysis machinery through a novel motif, the F-box. Cell $\underline{86}$, 263-274. 
Bálint, É. and Vousden, K.H., (2001). Activation and activities of the p53 tumour suppressor protein. Br. J. Cancer $\underline{85}$, 1813-1823.

Bayram, Ö. and Braus, G.H. (2012). Coordination of secondary metabolism and development in fungi: The velvet family of regulatory proteins. FEMS Microbiol. Rev. 36, 1-24.

Bayram, Ö., Feussner, K., Dumkow, M., Herrfurth, C., Feussner, I. and Braus, G.H. (2016). Changes of global gene expression and secondary metabolite accumulation during lightdependent Aspergillus nidulans development. Fungal Genet. Biol. 87, 30-53.

Bayram, Ö., Krappmann, S., Ni, M., Bok, J.W., Helmstaedt, K., Valerius, O., Braus-Stromeyer, S., Kwon, N.J., Keller, N.P., Yu, J.H. and Braus, G.H. (2008). VelB/VeA/LaeA complex coordinates light signal with fungal development and secondary metabolism. Science 320, $1504-1506$.

Bayram, Ö., Sarikaya-Bayram, Ö., Ahmed, Y.L., Maruyama, J.I., Valerius, O., Rizzoli, S.O., Ficner, R., Irniger, S. and Braus, G.H. (2012). The Aspergillus nidulans MAPK module AnSte11-Ste50-Ste7-Fus3 controls development and secondary metabolism. PLoS Genet. $\underline{8}$, e1002816. doi:10.1371/journal.pgen.1002816.

Beckmann, E.A., Köhler, A.M., Meister, C., Christmann, M., Draht, O.W., Rakebrandt, N., Valerius, O. and Braus, G.H. (2015). Integration of the catalytic subunit activates deneddylase activity in vivo as final step in fungal COP9 signalosome assembly. Mol. Microbiol. 97, 110-124.

Bellay, J., Han, S., Michaut, M., Kim, T.H., Costanzo, M., Andrews, B.J., Boone, C., Bader, G.D., Myers, C.L. and Kim, P.M. (2011). Bringing order to protein disorder through comparative genomics and genetic interactions. Genome Biol. 12, R14. doi: 10.1186/gb2011-12-2-r14.

Bergkessel, M. and Guthrie, C. (2013). Colony PCR. Methods Enzymol. 2ㅗ, 299-309.

Bhattacharyya, S., Yu, H., Mim, C. and Matouschek, A. (2014). Regulated protein turnover: Snapshots of the proteasome in action. Nat. Rev. Mol. Cell Biol. 15, 122-133.

Blumenstein, A., Vienken, K., Tasler, R., Purschwitz, J., Veith, D., Frankenberg-Dinkel, N. and Fischer, R. (2005). The Aspergillus nidulans phytochrome FphA represses sexual development in red light. Curr. Biol. 15, 1833-1838.

Bok, J.W., Chiang, Y.M., Szewczyk, E., Reyes-Domingez, Y., Davidson, A.D., Sanchez, J.F., Lo, H.C., Watanabe, K., Strauss, J., Oakley, B.R., Wang, C.C.C. and Keller, N.P. (2009). Chromatin-level regulation of biosynthetic gene clusters. Nat. Chem. Biol. $\underline{5}$, 462-464.

Bok, J.W. and Keller, N.P. (2004). LaeA, a regulator of secondary metabolism in Aspergillus spp. Eukaryot. Cell $\underline{3}, 527-535$.

Bok, J.W., Noordermeer, D., Kale, S.P. and Keller, N.P. (2006). Secondary metabolic gene cluster silencing in Aspergillus nidulans. Mol. Microbiol. 61, 1636-1645.

Bok, J.W., Soukup, A.A., Chadwick, E., Chiang, Y., Wang, C.C.C. and Keller, N.P. (2013). VeA 
and MvIA repression of the cryptic orsellinic acid gene cluster in Aspergillus nidulans

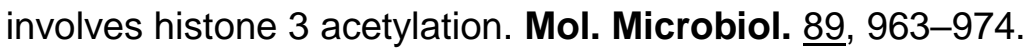

Borkovich, K.A., Alex, L.A., Yarden, O., Freitag, M., Turner, G.E., Read, N.D., Seiler, S., Bell-

Pedersen, D., Paietta, J., Plesofsky, N., Plamann, M., Goodrich-Tanrikulu, M., Schulte, U., Mannhaupt, G., Nargang, F.E., Radford, A., Selitrennikoff, C., Galagan, J.E., Dunlap, J.C., Loros, J.J., Catcheside, D., Inoue, H., Aramayo, R., Polymenis, M., Selker, E.U., Sachs, M.S., Marzluf, G. a, Paulsen, I., Davis, R., Ebbole, D.J., Zelter, A., Kalkman, E.R., O’Rourke, R., Bowring, F., Yeadon, J., Ishii, C., Suzuki, K., Sakai, W. and Pratt, R. (2004). Lessons from the genome analysis of Neurospora crassa. Microbiol. Mol. Biol. Rev. $\underline{68}, 1-108$.

Bos, J., Rehmann, H. and Wittinghofer, A. (2007). GEFs and GAPs: Critical elements in the control of small G proteins. Cell $\underline{129}$, 865-877.

Bosu, D.R. and Kipreos, E.T. (2008). Cullin-RING ubiquitin ligases: Global regulation and activation cycles. Cell Div. $\underline{3}$, doi: 10.1186/1747-1028-3-7.

Boylan, M.T., Mirabito, P.M., Willett, C.E., Zimmerman, C.R. and Timberlake, W.E. (1987). Isolation and physical characterization of three essential conidiation genes from Aspergillus nidulans. Mol. Cell. Biol. 푸, 3113-3118.

Brachmann, A. and Weinzierl, G. (2001). Identification of genes in the bW/bE regulatory cascade in Ustilago maydis. Mol. Microbiol. 42, 1047-1063.

Braga, G.U.L., Rangel, D.E.N., Fernandes, É.K.K., Flint, S.D. and Roberts, D.W. (2015). Molecular and physiological effects of environmental UV radiation on fungal conidia. Curr. Genet. $\underline{61}$, 405-425.

Brakhage, A.A. (2013). Regulation of fungal secondary metabolism. Nat. Rev. Microbiol. 11 , 21-32.

Brakhage, A.A., Browne, P. and Turner, G. (1992). Regulation of Aspergillus nidulans penicillin biosynthesis and penicillin biosynthesis genes acvA and ipnA by glucose. J. Bacteriol. 174, 3789-3799.

Braus, G.H., Krappmann, S. and Eckert, S.E. (2002). Sexual development in ascomycetes: Fruit body formation of Aspergillus nidulans. In: Osiewacz, H.D. (Ed.), Molecular Biology of Fungal Development. CRC Press, New York, pp. 215-244.

Brooke, A.G., (1994). Industrial fermentation and Aspergillus citric acid. In: Powell, K.A., Renwick, A.P. Eberdy, J.F. (Eds.), The Genus Aspergillus: From Taxonomy and Genetics to Industrial Application (F.E.M.S. Symposium Series). Springer US, Boston, MA, pp. 129-134.

Brown, C.J., Johnson, A.K., Dunker, A.K. and Daughdrill, G.W. (2009). Evolution and disorder. Curr. Opin. Struct. Biol. 21, 441-446.

Brown, S.H., Zarnowski, R., Sharpee, W.C. and Keller, N.P. (2008). Morphological transitions 
governed by density dependence and lipoxygenase activity in Aspergillus flavus. Appl.

Environ. Microbiol. 74, 5674-5685.

Buchanan, B.W., Lloyd, M.E., Engle, S.M. and Rubenstein, E.M. (2016). Cycloheximide chase analysis of protein degradation in Saccharomyces cerevisiae. J. Vis. Exp. 110, e53975.

Buljan, M., Chalancon, G., Dunker, A.K., Bateman, A., Balaji, S., Fuxreiter, M. and Babu, M.M. (2013). Alternative splicing of intrinsically disordered regions and rewiring of protein interactions. Curr. Opin. Struct. Biol. 23, 443-450.

Busch, S. and Braus, G.H. (2007). How to build a fungal fruit body: From uniform cells to specialized tissue. Mol. Microbiol. $\underline{64}$, 873-876.

Butnick, N.Z., Yager, L.N., Hermann, T.E., Kurtz, M.B. and Champe, S.P. (1984). Mutants of Aspergillus nidulans blocked at an early stage of sporulation secrete an unusual metabolite. J. Bacteriol. $\underline{160}$, 533-540.

Caballero-Lima, D., Kaneva, I.N., Watton, S.P., Sudbery, P.E. and Craven, C.J. (2013). The spatial distribution of the exocyst and actin cortical patches is sufficient to organize hyphal tip growth. Eukaryot. Cell 12, 998-1008.

Calvo, A.M., Lohmar, J.M., Ibarra, B. and Satterlee, T. (2016). Velvet regulation of fungal development. In: Wendland, J. (Ed.), The Mycota I: Growth, Differentiation and Sexuality. Springer International Publishing Switzerland, Cham, pp. 475-497.

Calvo, A.M., Wilson, R.A., Bok, J.W. and Keller, N.P. (2002). Relationship between secondary metabolism and fungal development. Microbiol. Biotechnol. Rev. $\underline{66}, 447-459$.

Canosa, I., Rojo, F. and Alonso, J.C., (1996). Site-specific recombination by the $\beta$ protein from the streptococcal plasmid pSM19035: Minimal recombination sequences and crossing over site. Nucleic Acids Res. 24, 2712-2717.

Cerqueira, G.C., Arnaud, M.B., Inglis, D.O., Skrzypek, M.S., Binkley, G., Simison, M., Miyasato, S.R., Binkley, J., Orvis, J., Shah, P., Wymore, F., Sherlock, G. and Wortman, J.R. (2014). The Aspergillus Genome Database: Multispecies curation and incorporation of RNA-Seq data to improve structural gene annotations. Nucleic Acids Res. $\underline{42}, 705-$ 710.

Chen, A.J., Frisvad, J.C., Sun, B.D., Varga, J., Kocsubé, S., Dijksterhuis, J., Kim, D.H., Hong, S.B., Houbraken, J. and Samson, R.A. (2016). Aspergillus section Nidulantes (formerly Emericella): Polyphasic taxonomy, chemistry and biology. Stud. Mycol. $\underline{84}, 1-118$.

Chen, H., Fujita, M., Feng, Q., Clardy, J. and Fink, G.R. (2004). Tyrosol is a quorum-sensing molecule in Candida albicans. Proc. Natl. Acad. Sci. 101, 5048-5052.

Chen, J., Chen, K., Yuan, S., Peng, X., Fang, J., Wang, F., Cui, H., Chen, Z., Yuan, J. and Geng, Y. (2016). Effects of aflatoxin B1 on oxidative stress markers and apoptosis of spleens in broilers. Toxicol. Ind. Heal. 32, 278-284.

Chen, J.W., Romero, P., Uversky, V.N. and Dunker, A.K. (2006). Conservation of intrinsic 
disorder in protein domains and families: I. A database of conserved predicted disordered regions. J. Proteome Res. $\underline{5}$, 879-887.

Chen, L., Gilkes, D.M., Pan, Y., Lane, W.S. and Chen, J. (2005). ATM and Chk2-dependent phosphorylation of MDMX contribute to p53 activation after DNA damage. EMBO J. $\underline{24}$, 3411-3422.

Christianson, J.C. and Ye, Y. (2014). Cleaning up in the endoplasmic reticulum: Ubiquitin in charge. Nat. Struc. Mol. Biol. 21, 325-335.

Christmann, M., Schmaler, T., Gordon, C., Huang, X., Bayram, Ö., Schinke, J., Stumpf, S., Dubiel, W. and Braus, G.H. (2013). Control of multicellular development by the physically interacting deneddylases DEN1/DenA and COP9 signalosome. PLoS Genet. $\underline{9}$, 22-24.

Ciechanover, A. (1998). The ubiquitin-proteasome pathway: On protein death and cell life.

EMBO J. 17, 7151-7160.

Ciechanover, A. (2012). Intracellular protein degradation: From a vague idea through the lysosome and the ubiquitin-proteosome system and onto human diseases and drug targeting. Rambam Maimonides Med. J. $\underline{3}$, e0001. doi: 10.5041/RMMJ.10068.

Cox, J. and Mann, M. (2008). MaxQuant enables high peptide identification rates, individualized p.p.b. - range mass accuracies and proteome-wide protein quantification.

Nature Biotechnol. 26, 1367-1372.

da Fonseca, P.C.A., He, J. and Morris, E.P. (2012). Molecular model of the human 26S proteasome. Mol. Cell $\underline{46}, 54-66$.

Davey, N.E., Roey, K. Van, Weatheritt, R.J., Toedt, G., Uyar, B., Altenberg, B., Budd, A., Diella, F. and Gibson, T.J. (2012). Attributes of short linear motifs. Mol. Biosyst. $\underline{8}, 268$ 281.

de Castro, E., Sigrist, C.J.A., Gattiker, A., Bulliard, V., Langendijk-Genevaux, P.S., Gasteiger, E., Bairoch, A. and Hulo, N. (2006). ScanProsite: Detection of PROSITE signature matches and ProRule-associated functional and structural residues in proteins. Nucleic Acids Res. 34, 362-365.

de Vries, R.P., Riley, R., Wiebenga, A., Aguilar-Osorio, G., Amillis, S., Uchima, C.A., Anderluh, G., Asadollahi, M., Askin, M., Barry, K., Battaglia, E., Bayram, Ö., Benocci, T., BrausStromeyer, S.A., Caldana, C., Cánovas, D., Cerqueira, G.C., Chen, F., Chen, W., Choi, C., Clum, A., dos Santos, R.A.C., de Damásio, A.R.L., Diallinas, G., Emri, T., Fekete, E., Flipphi, M., Freyberg, S., Gallo, A., Gournas, C., Habgood, R., Hainaut, M., Harispe, M.L., Henrissat, B., Hildén, K.S., Hope, R., Hossain, A., Karabika, E., Karaffa, L., Karányi, Z., Kraševec, N., Kuo, A., Kusch, H., LaButti, K., Lagendijk, E.L., Lapidus, A., Levasseur, A., Lindquist, E., Lipzen, A., Logrieco, A.F., MacCabe, A., Mäkelä, M.R., Malavazi, I., Melin, P., Meyer, V., Mielnichuk, N., Miskei, M., Molnár, Á.P., Mulé, G., Ngan, C.Y., Orejas, M., Orosz, E., Ouedraogo, J.P., Overkamp, K.M., Park, H.S., Perrone, G., Piumi, F., Punt, 
P.J., Ram, A.F.J., Ramón, A., Rauscher, S., Record, E., Riaño-Pachón, D.M., Robert, V., Röhrig, J., Ruller, R., Salamov, A., Salih, N.S., Samson, R.A., Sándor, E., Sanguinetti, M., Schütze, T., Sepčić, K., Shelest, E., Sherlock, G., Sophianopoulou, V., Squina, F.M., Sun, H., Susca, A., Todd, R.B., Tsang, A., Unkles, S.E., van de Wiele, N., van Rossen-Uffink, D., Oliveira, J.V. de C., Vesth, T.C., Visser, J., Yu, J.H., Zhou, M., Andersen, M.R., Archer, D.B., Baker, S.E., Benoit, I., Brakhage, A.A., Braus, G.H., Fischer, R., Frisvad, J.C., Goldman, G.H., Houbraken, J., Oakley, B., Pócsi, I., Scazzocchio, C., Seiboth, B., VanKuyk, P.A., Wortman, J., Dyer, P.S. and Grigoriev, I. V. (2017). Comparative genomics reveals high biological diversity and specific adaptations in the industrially and medically important fungal genus Aspergillus. Genome Biol. 트, doi: 10.1186/s13059017-1151-0.

Deshaies, R.J. and Joazeiro, C.A.P. (2009). RING domain E3 ubiquitin ligases. Annu. Rev. Biochem. 78, 399-434.

Despot, D.J., Kocsubé, S., Bencsik, O., Kecskeméti, A., Szekeres, A., Vágvölgyi, C., Varga, J. and Klarić, M.Š., (2016). Species diversity and cytotoxic potency of airborne sterigmatocystin-producing Aspergilli from the section Versicolores. Sci. Total Environ. 562, 296-304.

Dias, L.M., dos Santos, B.V., Albuquerque, C.J.B., Baeta, B.E.L., Pasquini, D. and Baffi, M.A. (2017). Biomass sorghum as a novel substrate in solid state fermentation for the production of hemicellulases and cellulases by Aspergillus niger and A. fumigatus. $\mathbf{J}$. Appl. Microbiol. 10.1111/ja, doi: 10.1111/jam.13672.

Dinkel, H., Roey, K., Michael, S., Kumar, M., Uyar, B., Altenberg, B., Milchevskaya, V., Schneider, M., Kühn, H., Behrendt, A., Dahl, S.L., Damerell, V., Diebel, S., Kalman, S., Klein, S., Knudsen, A.C., Mäder, C., Merrill, S., Staudt, A., Thiel, V., Welti, L., Davey, N.E., Diella, F. and Gibson, T.J. (2016). ELM 2016 - Data update and new functionality of the eukaryotic linear motif resource. Nucleic Acids Res. 44, D294-D300.

Dodds, D.R. and Gross, R.A. (2007). Chemicals from biomass. Science 318, 1250-1251.

Draht, O.W., Busch, S., Hofmann, K., Braus-Stromeyer, S., Helmstaedt, K., Goldmann, G.H. and Braus, G.H. (2007). Amino acid supply of Aspergillus. In: Goldmann, G.H., Osmani, S.O. (Eds.), The Aspergilli: Genomics, Medical Aspects, Biotechnology and Research Methods. CRC Press Taylor \& Francis Group, Boca Raton, FL, pp. 143-175.

Duda, D.M., Borg, L.A., Scott, D.C., Hunt, H.W., Hammel, M. and Schulman, B.A. (2008). Structural insights into NEDD8 activation of Cullin-RING ligases: Conformational control of conjugation. Cell 134, 995-1006.

Dunker, A.K., Babu, M.M., Barbar, E., Blackledge, M., Bondos, S.E., Dosztányi, Z., Dyson, H.J., Forman-Kay, J., Fuxreiter, M., Gsponer, J., Han, K.H., Jones, D.T., Longhi, S., Metallo, S.J., Nishikawa, K., Nussinov, R., Obradovic, Z., Pappu, R.V., Rost, B., Selenko, 
P., Subramaniam, V., Sussman, J.L., Tompa, P. and Uversky, V.N. (2013). What's in a name? Why these proteins are intrinsically disordered. Intrin. Dis. Pr. 1 , e24157. doi: 10.4161/idp.24157.

Dunker, A.K., Brown, C.J., Lawson, J.D., lakoucheva, L.M. and Obradovic, Z. (2002). Intrinsic disorder and protein function. Biochemistry $\underline{41}$, 6573-6582.

Dunker, A.K., Cortese, M.S., Romero, P., lakoucheva, L.M. and Uversky, V.N. (2005). Flexible nets: The roles of intrinsic disorder in protein interaction networks. FEBS J. 272, 5129 5148.

Dyer, P.S. and O'Gorman, C.M. (2011). Sexual development and cryptic sexuality in fungi: Insights from Aspergillus species. FEMS Microbiol. Rev. 트, 165-192.

Dyrka, W., Lamacchia, M., Durrens, P., Kobe, B., Daskalov, A., Paoletti, M., Sherman, D.J. and Saupe, S.J. (2014). Diversity and variability of NOD-like receptors in fungi. Genome Biol. Evol. $\underline{6}$, 3137-3158.

Dyson, H.J. (2016). Making sense of intrinsically disordered proteins. Biophys. J. $\underline{110}, 1013$ 1016.

Dyson, H.J. and Wright, P.E. (2016). Role of intrinsic protein disorder in the function and interactions of the transcriptional coactivators CREB-binding Protein (CBP) and p300. J. Biol. Chem. 291, 6714-6722.

Enkhbayar, P., Kamiya, M., Osaki, M., Matsumoto, T. and Matsushima, N. (2004). Structural principles of leucine-rich repeat (LRR) proteins. Proteins Struct. Funct. Genet. $\underline{54}, 394$ 403.

Estiarte, N., Lawrence, C.B., Sanchis, V., Ramos, A.J. and Crespo-Sempere, A. (2016). LaeA and $\mathrm{VeA}$ are involved in growth morphology, asexual development, and mycotoxin production in Alternaria alternata. Int. J. Food Microbiol. 238, 153-164.

Fajardo-Somera, R.A., Jöhnk, B., Bayram, Ö., Valerius, O., Braus, G.H. and Riquelme, M. (2015). Dissecting the function of the different chitin synthases in vegetative growth and sexual development in Neurospora crassa. Fungal Genet. Biol. $\underline{75}$, 30-45.

Ferrer-Bonet, M. and Ruiz-Trillo, I. (2017). Capsaspora owczarzaki. Curr. Biol. 27, R829R830.

Fischer, R. (2002). Conidiation in Aspergillus nidulans. In: Osiewacz, H.D. (Ed.), Molecular Biology of Fungal Development. Dekker, New York, pp. 59-86.

Fischer, R., Zekert, N. and Takeshita, N., (2008). Polarized growth in fungi - interplay between the cytoskeleton, positional markers and membrane domains. Mol. Microbiol. $\underline{68}, 813$ 826.

Fishbain, S., Inobe, T., Israeli, E., Chavali, S., Yu, H., Kago, G., Babu, M.M. and Matouschek, A. (2015). Sequence composition of disordered regions fine-tunes protein half-life. Nat. Struct. Mol. Biol. 22, 214-221. 
Frisvad, J.C. and Thrane, U.L.F. (1987). Standardized high-performance liquid chromatography of 182 mycotoxins and other fungal metabolites based on alkylphenone retention indices and UV-VIS spectra (diode array detection). J. Chromatogr. 404, 195214.

Fuxreiter, M., Simon, I. and Bondos, S. (2011). Dynamic protein-DNA recognition: Beyond what can be seen. Trends Biochem. Sci. 36, 415-423.

Galagan, J.E., Calvo, S.E., Cuomo, C., Ma, L., Wortman, J.R., Batzoglou, S., Spevak, C.C., Clutterbuck, J., Kapitonov, V., Jurka, J., Scazzocchio, C., Farman, M., Butler, J., Purcell, S., Harris, S., Braus, G.H., Draht, O., Busch, S., Enfert, C.D., Bouchier, C., Goldman, G.H., Denning, D.W., Caddick, M., Hynes, M., Paoletti, M., Fischer, R., Miller, B., Dyer, P., Sachs, M.S., Osmani, S.A. and Birren, B.W., (2005). Sequencing of Aspergillus nidulans and comparative analysis with $A$. fumigatus and $A$. oryzae. Nature $\underline{438}, 1105$ 1115.

Gao, J.X., Yu, C.I., Wang, M., Sun, J.N., Li, Y.Q. and Chen, J. (2017). Involvement of a velvet protein CIVeIB in the regulation of vegetative differentiation, oxidative stress response, secondary metabolism, and virulence in Curvularia lunata. Sci. Rep. $\underline{7}$, doi: $10.1038 / \mathrm{srep} 46054$.

Garviea, C.W. and Wolberger, C. (2001). Recognition of specific DNA sequences. Mol. Cell $\underline{8}$ 937-946.

Garzia, A., Etxebeste, O., Herrero-García, E., Ugalde, U. and Espeso, E.A. (2010). The concerted action of bZip and cMyb transcription factors FlbB and FlbD induces brlA expression and asexual development in Aspergillus nidulans. Mol. Microbiol. $\underline{75}, 1314$ 1324.

Garzia, A., Etxebeste, O., Rodríguez-Romero, J., Fischer, R., Espeso, E.A. and Ugalde, U. (2013). Transcriptional changes in the transition from vegetative cells to asexual development in the model fungus Aspergillus nidulans. Eukaryot. Cell 12, 311-321.

Gerke, J., Bayram, Ö., Feussner, K., Landesfeind, M., Shelest, E., Feussner, I. and Braus, G.H. (2012). Breaking the silence: Protein stabilization uncovers silenced biosynthetic gene clusters in the fungus Aspergillus nidulans. Appl. Environ. Microbiol. $\underline{78}, 8234$ 8244.

Gerke, J. and Braus, G.H. (2014). Manipulation of fungal development as source of novel secondary metabolites for biotechnology. Appl. Microbiol. Biotechnol. $\underline{98}, 8443-8455$.

Glickman, M.H. and Ciechanover, A. (2002). The ubiquitin-proteasome proteolytic pathway:

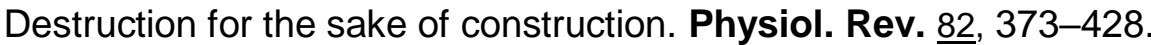

Gouw, M., Sámano-Sánchez, H., Van Roey, K., Diella, F., Gibson, T.J. and Dinkel, H. (2017). Exploring short linear motifs using the ELM database and tools. Curr. Protoc. Bioinforma. $\underline{58}$, 8.22.1-8.22.35, doi: 10.1002/cpbi.26. 
Gsponer, J. and Babu, M.M. (2009). The rules of disorder or why disorder rules. Prog. Biophys. Mol. Biol. 99, 94-103.

Guo, X., Bulyk, M.L. and Hartemink, A.J. (2012). Intrinsic disorder within and flanking the DNAbinding domains of human transcription factors. Pac. Symp. Biocomput. 104-115.

Hampel, M., Jakobi, M., Schmitz, L., Meyer, U., Finkernagel, F., Doehlemann, G. and Heimel, K. (2016). Unfolded protein response (UPR) regulator Cib1 controls expression of genes encoding secreted virulence factors in Ustilago maydis. PLoS One 11, e0153861. doi: 10.1371/journal.pone.0153861.

Han, K.H. (2009). Molecular genetics of Emericella nidulans sexual development. Mycobiology $\underline{37}, 171-182$.

Hanahan, D. (1985). Techniques for transformation of E. coli. In: Glover, D.M. (Ed.), DNA cloning: a practical approach Vol. 1. IRL Press, Oxford, pp. 109-135.

Hanahan, D., Jessee, J. and Bloom, F.R. (1991). Plasmid transformation of Escherichia coli and other bacteria. Methods Enzymol. 204, 63-113.

Harris, S.D. (2009). The Spitzenkörper: A signalling hub for the control of fungal development? Mol. Microbiol. $\underline{73}, 733-736$.

Harrison, S.C., (1991). A structural taxonomy of DNA-binding domains. Nature $\underline{353}, 715-719$.

Hartmann, T., Dümig, M., Jaber, B.M., Szewczyk, E., Olbermann, P., Morschhäuser, J. and Krappmann, S. (2010). Validation of a self-excising marker in the human pathogen Aspergillus fumigatus by employing the $\beta$-rec/six site-specific recombination system. Appl. Environ. Microbiol. 트, 6313-6317.

Haynes, C., Oldfield, C.J., Ji, F., Klitgord, N., Cusick, M.E., Radivojac, P., Uversky, V.N., Vidal, M. and lakoucheva, L.M. (2006). Intrinsic disorder is a common feature of hub proteins from four eukaryotic interactomes. PLoS Comput. Biol. 2 10.1371/journal.pcbi.0020100.

He, C. and Klionsky, D.J. (2009). Regulation mechanisms and signalling pathways of autophagy. Annu. Rev. Genet. $\underline{43}, 67-93$.

He, Q., Cheng, P., Yang, Y., He, Q., Yu, H. and Liu, Y. (2003). FWD1-mediated degradation of FREQUENCY in Neurospora establishes a conserved mechanism for circadian clock regulation. EMBO J. 22, $4421-4430$.

Heimel, K. (2014). Unfolded protein response in filamentous fungi - implications in biotechnology. Appl. Microbiol. Biotechnol. $\underline{99}$, 121-132.

Heimel, K., Scherer, M., Schuler, D. and Kamper, J. (2010). The Ustilago maydis Clp1 protein orchestrates pheromone and $b$-dependent signaling pathways to coordinate the cell cycle and pathogenic development. Plant Cell 22, 2908-2922.

Heinemeyer, T., Chen, X., Karas, H., Kel, A.E., Kel, O. V., Liebich, I., Meinhardt, T., Reuter, I., Schacherer, F. and Wingender, E. (1999). Expanding the TRANSFAC database towards 
an expert system of regulatory molecular mechanisms. Nucleic Acids Res. 27, 318-322. Helmstaedt, K., Schwier, E.U., Christmann, M., Nahlik, K., Westermann, M., Harting, R., Grond, S., Busch, S. and Braus, G.H. (2011). Recruitment of the inhibitor Cand1 to the cullin substrate adaptor site mediates interaction to the neddylation site. Mol. Biol. Cell 22, 153-164.

Hermann, T.E., Kurtz, M.B. and Champe, S.P. (1983). Laccase localized in Hulle cells and cleistothecial primordia of Aspergillus nidulans. J. Bacteriol. 154, 955-964.

Herrero-Garcia, E., Perez-de-Nanclares-Arregi, E., Cortese, M.S., Markina-Iñarrairaegui, A., Oiartzabal-Arano, E., Etxebeste, O., Ugalde, U. and Espeso, E.A. (2015). Tip-to-nucleus migration dynamics of the asexual development regulator FlbB in vegetative cells. Mol. Microbiol. 98, 607-624.

Hersko, A. and Ciechanover, A. (1998). The ubiquitin system. Annu. Rev. Biochem. $\underline{67}$, 425479.

Hess, J. (2004). AP-1 subunits: quarrel and harmony among siblings. J. Cell Sci. 117, 59655973.

Hibino, E., Inoue, R., Sugiyama, M., Kuwahara, J., Matsuzaki, K. and Hoshino, M. (2016) Interaction between intrinsically disordered regions in transcription factors Sp1 and TAF4. Protein Sci. 25, 2006-2017.

Hiramatsu, N., Hibino, E., Matsuzaki, K., Kuwahara, J. and Hoshino, M. (2012). Interaction between isolated transcriptional activation domains of Sp1 revealed by heteronuclear magnetic resonance. Protein Sci. 21, 1481-1488.

Hirsch, C., Gauss, R., Horn, S.C., Neuber, O. and Sommer, T. (2009). The ubiquitylation machinery of the endoplasmic reticulum. Nature $458,453-460$.

Ho, M.S., Tsai, P.I. and Chien, C.T. (2006). F-box proteins: The key to protein degradation. J. Biomed. Sci. 13, 181-191.

Hoffmann, B., Wanke, C., LaPaglia, S.K. and Braus, G.H. (2000). c-Jun and RACK1 homologues regulate a control point for sexual development in Aspergillus nidulans. Mol. Microbiol. 37, 28-41.

Hofmann, M.A. and Brian, D.A. (1991). Sequencing PCR DNA amplified directly from a bacterial colony. Biotechniques 11, 30-31.

Hong, W., Jiao, W., Hu, J., Zhang, J., Liu, C., Fu, X., Shen, D., Xia, B. and Chang, Z. (2005). Periplasmic protein HdeA exhibits chaperone-like activity exclusively within stomach $\mathrm{pH}$ range by transforming into disordered conformation. J. Biol. Chem. 280, 27029-27034. Hornby, J.M., Jensen, E.C., Lisec, A.D., Tasto, J., Jahnke, B., Shoemaker, R., Nickerson, K.W., Tasto, J.J. and Dussault, P. (2001). Quorum sensing in the dimorphic fungus Candida albicans is mediated by farnesol. Appl. Environ. Microbiol. 67, 2982-2992.

Hu, G., Wu, Z., Uversky, V. and Kurgan, L. (2017). Functional analysis of human hub proteins 
and their interactors involved in the intrinsic disorder-enriched interactions. Int. J. Mol. Sci. 18, pii: E2761. doi: 10.3390/ijms18122761.

Hua, Z. and Vierstra, R.D. (2011). The Cullin-RING ubiquitin-protein ligases. Annu. Rev. Plant Biol. $\underline{62}$, 299-334.

lakoucheva, L.M., Radivojac, P., Brown, C.J., O’Connor, T.R., Sikes, J.G., Obradovic, Z. and Dunker, A.K. (2004). The importance of intrinsic disorder for protein phosphorylation. Nucleic Acids Res. 32, 1037-1049.

Ibarra, B.A., Lohmar, J.M., Satterlee, T., McDonald, T., Cary, J.W. and Calvo, A.M. (2017). The 14-3-3 homolog, ArtA, regulates development and secondary metabolism in the opportunistic plant pathogen Aspergillus flavus. Appl. Environ. Microbiol. pii: AEM.02241-17. doi: 10.1128/AEM.02241-17.

Inoue, H., Nojima, H. and Okayama, H. (1990). High efficiency transformation of Escherichia coli with plasmids. Gene $\underline{96}, 23-28$.

Irniger, S. and Braus, G.H. (2003). Controlling transcription by destruction: the regulation of yeast Gcn4p stability. Curr. Genet. $\underline{44}, 8-18$.

Itoh, E., Shigemoto, R., Oinuma, K.I., Shimizu, M., Masuo, S. and Takaya, N. (2017). Sirtuin A regulates secondary metabolite production by Aspergillus nidulans. J. Gen. Appl. Microbiol. 235, 228-235.

Jakob, U., Kriwacki, R. and Uversky, V.N. (2014). Conditionally and transiently disordered proteins: Awakening cryptic disorder to regulate protein function. Chem. Rec. 114, 67796805.

Jin, J., Cardozo, T., Lovering, R.C., Elledge, S.J., Pagano, M. and Harper, J.W. (2004). Systematic analysis and nomenclature of mammalian F-box proteins. Genes Dev. $\underline{18}$, 2573-2580

John, M., Leppik, R., Busch, S.J., Granger-Schnarr, M. and Schnarr, M. (1996). DNA binding of Jun and Fos bZip domains: Homodimers and heterodimers induce a DNA conformational change in solution. Nucleic Acids Res. 24, 4487-4494.

Jöhnk, B., Bayram, Ö., Abelmann, A., Heinekamp, T., Mattern, D.J., Brakhage, A.A., Jacobsen, I.D., Valerius, O. and Braus, G.H. (2016). SCF ubiquitin ligase F-box protein Fbx15 controls nuclear co-repressor localization, stress response and virulence of the human pathogen Aspergillus fumigatus. PLoS Pathog. 12, e1005899. doi: 10.1371/journal.ppat.1005899.

Jones, P., Binns, D., Chang, H.Y., Fraser, M., Li, W., McAnulla, C., McWilliam, H., Maslen, J., Mitchell, A., Nuka, G., Pesseat, S., Quinn, A.F., Sangrador-Vegas, A., Scheremetjew, M., Yong, S.Y., Lopez, R. and Hunter, S. (2014). InterProScan 5: Genome-scale protein function classification. Bioinformatics 30, 1236-1240.

Jonkers, W. and Rep, M. (2009). Lessons from fungal F-Box proteins. Eukaryot. Cell $\underline{8}$, 677- 
695.

Käfer, E. (1965). Origins of translocations in Aspergillus nidulans. Genetics $\underline{52}$, 217-232.

Kämper, J., Reichmann, M., Romeis, T., Bölker, M. and Kahmann, R. (1995). Multiallelic recognition: Nonself-dependent dimerization of the bE and bW homedomain proteins in Ustilago maydis. Cell 81, 73-83.

Kang, D., Gho, Y.S., Suh, M. and Kang, C. (2002). Highly sensitive and fast protein detection with Coomassie Brilliant Blue in sodium dodecyl sulfate-polyacrylamide gel electrophoresis. Bull. Korean Chem. Soc. 23, 1511-1512.

Kang, H.J. and Rosenwaks, Z., (2018). p53 and reproduction. Fertil. Steril. 109, 39-43.

Kato, N., Brooks, W. and Calvo, A.M. (2003). The expression of sterigmatocystin and penicillin genes in Aspergillus nidulans is controlled by $v e A$, a gene required for sexual development. Eukaryot. Cell $\underline{2}, 1178-1186$.

Kay, B.K., Williamson, M.P. and Sudol, M. (2000). The importance of being proline: The interaction of proline-rich motifs in signaling proteins with their cognate domains. FASEB J. $14,231-241$.

Kempken, F. (2011). Fungal defences against animal antagonists - lectins \& more. Mol. Ecol. 20, 2876-2877.

Kibbe, W.A. (2007). OligoCalc: An online oligonucleotide properties calculator. Nucleic Acids Res. $\underline{35}, 43-46$.

Kim, H.S., Han, K.Y., Kim, K.J., Han, D.M., Jahng, K.Y. and Chae, K.S. (2002). The veA gene activates sexual development in Aspergillus nidulans. Fungal Genet. Biol. 37, 72-80.

Kirkin, V., McEwan, D.G., Novak, I. and Dikic, I. (2009). A role for ubiquitin in selective autophagy. Mol. Cell 34, 259-269.

Kleiger, G. and Mayor, T. (2015). Perilous journey: A tour of the ubiquitin-proteasome system. Trends Cell Biol. 24, 352-359.

Kleinknecht, A., Popova, B., Lázaro, D.F., Pinho, R., Valerius, O., Outeiro, T.F. and Braus, G.H. (2016). C-terminal tyrosine residue modifications modulate the protective phosphorylation of serine 129 of $\alpha$-synuclein in a yeast model of Parkinson's disease. PLoS Genet. 12, e1006098. doi: 10.1371/journal.pgen.1006098.

Kobe, B. and Deisenhofer, J. (1994). The leucine-rich repeat: A versatile binding motif. Trends Biochem. Sci. 19, 415-421.

Kolog Gulko, M., Heinrich, G., Gross, C., Popova, B., Valerius, O., Neumann, P., Ficner, R. and Braus, G.H. (2018). Sem1 links proteasome stability and specificity to multicellular development. PLoS Genet. accepted.

Komander, D. and Rape, M. (2012). The ubiquitin code. Annu. Rev. Biochem. 81, 203-229.

Koressaar, T. and Remm, M., (2007). Enhancements and modifications of primer design program Primer3. Bioinformatics $\underline{23}, 1289-1291$. 
Kosugi, S., Hasebe, M., Tomita, M. and Yanagawa, H. (2009). Systematic identification of cell cycle-dependent yeast nucleocytoplasmic shuttling proteins by prediction of composite motifs. Proc. Natl. Acad. Sci. 106, 10171-10176.

Kragelund, B.B., Schenstrøm, S.M., Rebula, C.A., Panse, V.G. and Hartmann-Petersen, R. (2016). DSS1/Sem1, a multifunctional and intrinsically disordered protein. Trends Biochem. Sci. 41, 446-459.

Krappmann, S., Bignell, E.M., Reichard, U., Rogers, T., Haynes, K. and Braus, G.H. (2004).

The Aspergillus fumigatus transcriptional activator $\mathrm{CpcA}$ contributes significantly to the virulence of this fungal pathogen. Mol. Microbiol. $\underline{52}, 785-799$.

Krappmann, S., Jung, N., Medic, B., Busch, S., Prade, R.A. and Braus, G.H. (2006a). The Aspergillus nidulans F-box protein GrrA links SCF activity to meiosis. Mol. Microbiol. $\underline{61}$, 76-88.

Krappmann, S., Sasse, C. and Braus, G.H. (2006b). Gene targeting in Aspergillus fumigatus by homologous recombination is facilitated in a nonhomologous end-joining-deficient genetic background. Eukaryot. Cell $\underline{5}, 212-215$.

Kumar, D., Barad, S., Chen, Y., Luo, X., Tannous, J., Dubey, A., Glam Matana, N., Tian, S., Li, B., Keller, N.P. and Prusky, D. (2016). LaeA regulation of secondary metabolism modulates virulence in Penicillium expansum and is mediated by sucrose. Mol. Plant Pathol. 18, 1150-1163.

Kurtz, M.B. and Champe, S.P. (1981). Dominant spore color mutants of Aspergillus nidulans defective in germination and sexual development. J. Bacteriol. 148, 629-638.

Kwon, Y.T. and Ciechanover, A. (2017). The ubiquitin code in the ubiquitin-proteasome system and autophagy. Trends Biochem. Sci. 42, 873-886.

Laemmli, U.K. (1970). Cleavage of structural proteins during the assembly of the head of bacteriophage T4. Nature 227, 680-685.

Latysheva, N.S., Flock, T., Weatheritt, R.J., Chavali, S. and Babu, M.M. (2015). How do disordered regions achieve comparable functions to structured domains? Protein Sci. 24 909-922.

Lee, B.N. and Adams, T.H. (1994). The Aspergillus nidulans fluG gene is required for production of an extracellular developmental signal and is related to prokaryotic glutamine synthetase I. Genes Dev. 무, 641-651.

Lee, M.K., Kwon, N.J., Choi, J.M., Lee, I.S., Jung, S. and Yu, J.H. (2014). NsdD is a key repressor of asexual development in aspergillus nidulans. Genetics 197, 159-173.

Lee, M.K., Kwon, N.J., Lee, I.S., Jung, S., Kim, S.C. and Yu, J.H. (2016). Negative regulation and developmental competence in Aspergillus. Sci. Rep. $\underline{6}$, doi:10.1038/srep28874.

Lee, P.Y., Costumbrado, J., Hsu, C.Y. and Kim, Y.H. (2012). Agarose gel electrophoresis for the separation of DNA fragments. J. Vis. Exp. $\underline{62}$, pii: 3923. doi: 10.3791/3923. 
Lee, S.B. and Taylor, J.W. (1990). Isolation of DNA from fungal mycelia and single spores. In: Innis, N., Gelfand, D., Sninsky, J., White, T. (Eds.), PCR Protocols: A Guide to Methods and Applications. Academic Press, New York, pp. 282-287.

Lee, R. Van Der, Buljan, M., Lang, B., Weatheritt, R.J., Daughdrill, G.W., Dunker, A.K., Fuxreiter, M., Gough, J., Gsponer, J., Jones, D.T., Kim, P.M., Kriwacki, R.W., Old, C.J., Pappu, R. V, Tompa, P., Uversky, V.N., Wright, P.E. and Babu, M.M. (2015). Classification of intrinsically disordered regions and proteins. Chem. Rev. $\underline{114}$, 65896631.

Li, W., Cowley, A., Uludag, M., Gur, T., McWilliam, H., Squizzato, S., Park, Y.M., Buso, N. and Lopez, R. (2015). The EMBL-EBI bioinformatics web and programmatic tools framework. Nucleic Acids Res. 43, W580-W584.

Liu, J., Perumal, N.B., Oldfield, C.J., Su, E.W., Uversky, V.N. and Dunker, A.K. (2006). Intrinsic disorder in transcription factors. Biochemistry $\underline{45}$, 6873-6888.

Lo, H., Entwistle, R., Guo, C., Ahuja, M., Szewczyk, E., Hung, J., Chiang, Y., Oakley, B.R. and Wang, C.C.C. (2012). Two separate gene clusters encode the biosynthetic pathway for the meroterpenoids austinol and dehydroaustinol in Aspergillus nidulans. J. Am. Chem. Soc. $134,4709-4720$.

Lobley, A., Swindells, M.B., Orengo, C.A. and Jones, D.T. (2007). Inferring function using patterns of native disorder in proteins. PLoS Comput. Biol. $\underline{3}, 1567-1579$.

López-Díaz, C., Rahjoo, V., Sulyok, M., Ghionna, V., Martín-Vicente, A., Capilla, J., Di Pietro, A. and López-Berges, M.S. (2017). Fusaric acid contributes to virulence of Fusarium oxysporum on plant and mammalian hosts. Mol. Plant Pathol. 19, 440-453.

Mabey Gilsenan, J., Cooley, J. and Bowyer, P. (2012). CADRE: The Central Aspergillus Data REpository 2012. Nucleic Acids Res. 40, 660-666.

Macheleidt, J., Mattern, D.J., Fischer, J., Netzker, T., Weber, J., Schroeckh, V., Valiante, V. and Brakhage, A.A. (2016). Regulation and role of fungal secondary metabolites. Annu. Rev. Genet. 50, 371-392.

Macias, M.J., Hyvönen, M., Baraldi, E., Schultz, J., Sudol, M., Saraste, M. and Oschkinat, H. (1996). Structure of the WW domain of a kinase-associated protein complexed with a proline-rich peptide. Nature $\underline{382}, 646-649$.

Macias, M.J., Wiesner, S. and Sudol, M. (2002). WW and SH3 domains, two different scaffolds to recognize proline-rich ligands. FEBS Lett. $\underline{513}$, 30-37.

Mao, A.H., Lyle, N. and Pappu, R.V. (2013). Describing sequence-ensemble relationships for intrinsically disordered proteins. Biochem. J. $\underline{449}$, 307-318.

Mao, X., Cao, F., Nie, X., Liu, H. and Chen, J., (2006). The Swi/Snf chromatin remodeling complex is essential for hyphal development in Candida albicans. FEBS Lett. $\underline{580}$, 26152622. 
Marchler-Bauer, A., Bo, Y., Han, L., He, J., Lanczycki, C.J., Lu, S., Chitsaz, F., Derbyshire, M.K., Geer, R.C., Gonzales, N.R., Gwadz, M., Hurwitz, D.I., Lu, F., Marchler, G.H., Song, J.S., Thanki, N., Wang, Z., Yamashita, R.A., Zhang, D., Zheng, C., Geer, L.Y. and Bryant, S.H. (2017). CDD/SPARCLE: Functional classification of proteins via subfamily domain architectures. Nucleic Acids Res. 45, D200-D203.

Martens, J.A. and Winston, F. (2003). Recent advances in understanding chromatin remodeling by Swi/Snf complexes. Curr. Opin. Genet. Dev. 13, 136-142.

McCluskey, K., Wiest, A. and Plamann, M. (2010). The fungal genetics stock center: A repository for 50 years of fungal genetics research. J. Biosci. 35, 119-126.

McWilliam, H., Li, W., Uludag, M., Squizzato, S., Park, Y.M., Buso, N., Cowley, A.P. and Lopez, R. (2013). Analysis tool web services from the EMBL-EBI. Nucleic Acids Res. 41 W597-W600.

Meister, C., Kolog Gulko, M., Köhler, A.M. and Braus, G.H. (2016). The devil is in the details: Comparison between COP9 signalosome (CSN) and the LID of the $26 \mathrm{~S}$ proteasome. Curr. Genet. 62, 129-136.

Mims, C.W., Richardson, E.A. and Timberlake, W.E. (1988). Ultrastructural analysis of conidiophore development in the fungus Aspergillus nidulans. Protoplasma $\underline{144}, 132$ 141.

Mitchell, S., Vargas, J. and Hoffmann, A. (2016). Signaling via the NFkB system. WIREs Syst. Biol. Med. $\underline{8}, 227-241$.

Mooney, J.L., Hassett, D.E. and Yager, L.N. (1990). Genetic analysis of suppressors of the veA1 mutation in Aspergillus nidulans. Genetics $\underline{126}$, 869-874.

Mooney, J.L. and Yager, L.N. (1990). Light is required for conidiation in Aspergillus nidulans. Genes Dev. 4, 1473-1482.

Mughal, M.J., Peng, X., Zhou, Y. and Fang, J. (2016). Aflatoxin B 1 invokes apoptosis via death receptor pathway in hepatocytes. Oncotarget $\underline{8}, 8239-8249$.

Müller, C.W., Rey, F.A., Sodeoka, M., Verdine, G.L. and Harrison, S.C. (1995). Structure of the NF-kB p50 homodimer bound to DNA. Nature $\underline{373}, 311-317$.

Nahlik, K., Dumkow, M., Bayram, Ö., Helmstaedt, K., Busch, S., Valerius, O., Gerke, J., Hoppert, M., Schwier, E., Opitz, L., Westermann, M., Grond, S., Feussner, K., Goebel, C., Kaever, A., Meinicke, P., Feussner, I. and Braus, G.H. (2010). The COP9 signalosome mediates transcriptional and metabolic response to hormones, oxidative stress protection and cell wall rearrangement during fungal development. Mol. Microbiol. $\underline{78}$, 964-979.

Nam, T., Han, J.H., Devkota, S. and Lee, and H.W. (2017). Emerging paradigm of crosstalk between autophagy and the ubiquitin-proteasome system. Mol. Cells 40, 897-905.

Nayak, T., Szewczyk, E., Oakley, C.E., Osmani, A., Ukil, L., Murray, S.L., Hynes, M.J., Osmani, S.A. and Oakley, B.R. (2005). A versatile and efficient gene-targeting system for 
Aspergillus nidulans. Genetics $\underline{172}, 1557-1566$.

Neer, E.J., Schmidt, C.J., Nambudripad, R. and Smith, T.F. (1994). The ancient regulatoryprotein family of WD-repeat proteins. Nature $\underline{371}, 297-300$.

$\mathrm{Ni}, \mathrm{M}$. and Yu, J.H. (2007). A novel regulator couples sporogenesis and trehalose biogenesis in Aspergillus nidulans. PLoS One 2, e970. doi:10.1371/journal.pone.0000970.

Nielsen, M.L., Nielsen, J.B., Rank, C., Klejnstrup, M.L., Holm, D.K., Brogaard, K.H., Hansen, B.G., Frisvad, J.C., Larsen, T.O. and Mortensen U.H. (2011). A genome-wide polyketide synthase deletion library uncovers novel genetic links to polyketides and meroterpenoids in Aspergillus nidulans. FEMS Microbiol. Lett. $\underline{321}$, 157-166.

O'Dea, E. and Hoffmann, A. (2010). The regulatory logic of the NF-kB signaling system. Cold Spring Harb. Perspect. Med. 2, a000216. doi: 10.1101/cshperspect.a000216.

Oakley, C.E., Ahuja, M., Sun, W.W., Entwistle, R., Akashi, T., Yaegashi, J., Guo, C.J., Cerqueira, G.C., Russo Wortman, J., Wang, C.C.C., Chiang, Y.M. and Oakley, B.R. (2017). Discovery of McrA, a master regulator of Aspergillus secondary metabolism. Mol. Microbiol. 103, 347-365.

Oeckeninghaus, A., Hayden, M.S. and Ghosh, S. (2011). Crosstalk in NF-kB signaling pathways. Nat. Immunol. 12, 695-708.

Oiartzabal-Arano, E., Perez-de-Nanclares-Arregi, E., Espeso, E.A. and Etxebeste, O. (2016). Apical control of conidiation in Aspergillus nidulans. Curr. Genet. $\underline{62}, 371-377$.

Oldfield, C.J., Meng, J., Yang, J.Y., Qu, M.Q., Uversky, V.N. and Dunker, A.K. (2008). Flexible nets: Disorder and induced fit in the associations of p53 and 14-3-3 with their partners. BMC Genomics $\underline{9}$, doi: 10.1186/1471-2164-9-S1-S1.

Olma, M.H. and Dikic, I. (2013). Cullins getting undressed by the protein exchange factor Cand1. Cell 153, 14-16.

Onodera, J. and Ohsumi, Y. (2005). Autophagy is required for maintenance of amino acid levels and protein synthesis under nitrogen starvation. J. Biol. Chem. 280, 31582-31586.

Ota, M., Gonja, H., Koike, R. and Fukuchi, S. (2016). Multiple-localization and hub proteins. PLoS One 11, e0156455. doi: 10.1371/journal.pone.0156455.

Palmer, J.M., Theisen, J.M., Duran, R.M., Grayburn, W.S., Calvo, A.M. and Keller, N.P. (2013). Secondary metabolism and development is mediated by LImF control of VeA subcellular localization in Aspergillus nidulans. PLoS Genet. $\underline{9}$, e1003193. doi: 10.1371/journal.pgen.1003193.

Paluh, J.L., Orbach, M.J., Legerton, T.L. and Yanofsky, C. (1988). The cross-pathway control gene of Neurospora crassa, cpc-1, encodes a protein similar to GCN4 of yeast and the DNA-binding domain of the oncogene v-jun-encoded protein. Proc. Natl. Acad. Sci. USA 85, 3728-3732.

Paluh, J.L. and Yanofsky, C. (1991). Characterization of Neurospora CPC1, a bZIP DNA- 
binding protein that does not require aligned heptad leucines for dimerization. Mol. Cell. Biol. 11, 935-44.

Park, H.S., Bayram, Ö., Braus, G.H., Kim, S.C. and Yu, J.H. (2012a). Characterization of the velvet regulators in Aspergillus fumigatus. Mol. Microbiol. 86, 937-953.

Park, H.S., Lee, M.K., Kim, S.C. and Yu, J.H. (2017). The role of VosA/VelB-activated developmental gene vadA in Aspergillus nidulans PLoS One 12, e0177099. doi: 10.1371/journal.pone.0177099.

Park, H.S., Man Yu, Y., Lee, M.K., Jae Maeng, P., Chang Kim, S. and Yu, J.H. (2015). Velvetmediated repression of $\beta$-glucan synthesis in Aspergillus nidulans spores. Sci. Rep. $\underline{5}$, 10199. doi: 10.1038/srep10199.

Park, H.S., Nam, T.Y., Han, K.H., Kim, S.C. and Yu, J.H. (2014). VelC positively controls sexual development in Aspergillus nidulans. PLoS One $\underline{9}$, e89883. doi:10.1371/journal.pone.0089883.

Park, H.S., Ni, M., Jeong, K.C., Kim, Y.H. and Yu, J.H. (2012b). The role, interaction and regulation of the velvet regulator VeIB in Aspergillus nidulans. PLoS One U, e45935. doi: 10.1371/journal.pone.0045935.

Park, H.S. and Yu, J.H. (2012). Genetic control of asexual sporulation in filamentous fungi. Curr. Opin. Microbiol. 15, 669-677.

Patel, L., Abate, C. and Curran, T. (1990). Altered protein conformation on DNA binding by Fos and Jun. Nature 347, 572-575.

Patil, A., Kinoshita, K. and Nakamura, H. (2010). Hub promiscuity in protein-protein interaction networks. Int. J. Mol. Sci. 11, 1930-1943.

Pereira, J.H. and Kim, S.H. (2009). Structure of human Brn-5 transcription factor in complex with CRH gene promoter. J. Struct. Biol. 167, 159-165.

Peters, J.M., Franke, W.W. and Kleinschmidt, J.A. (1994). Distinct $19 S$ and 20 S subcomplexes of the $26 \mathrm{~S}$ proteasome and their distribution in the nucleus and the cytoplasm. J. Biol. Chem. 269, 7709-7718.

Petroski, M.D. and Deshaies, R.J. (2005). Function and regulation of cullin-RING ubiquitin ligases. Nat. Rev. Mol. Cell Biol. $\underline{6}$, 9-20.

Pöggeler, S., Nowrousian, M. and Kück, U. (2006). Fruiting-body development in ascomycetes. In: Kues, U., Fischer, R. (Eds.), The Mycota I: Growth, Differentiation and Sexuality. Springer-Verlag, Heideberg, pp. 325-355.

Potoyan, D.A., Bueno, C., Zheng, W., Komives, E.A. and Wolynes, P.G. (2017). Resolving the NFKB hetero-dimer binding paradox: Strain and frustration guide the binding of dimeric transcription factors. J. Am. Chem. Soc. 139, 18558-18566.

Punt, P.J. and van den Hondel, C.A. (1992). Transformation of filamentous fungi based on hygromycin b and phleomycin resistance markers. Methods Enzymol. 216, 447-457. 
Purschwitz, J., Müller, S., Kastner, C., Schöser, M., Haas, H., Espeso, E.A., Atoui, A., Calvo, A.M. and Fischer, R. (2008). Functional and physical interaction of blue- and red-light sensors in Aspergillus nidulans. Curr. Biol. 18, 255-259.

Rappsilber, J., Ishihama, Y. and Mann, M. (2003). Stop and go extraction tips for matrixassisted laser desorption/ionization, nanoelectrospray, and LC/MS sample pretreatment in proteomics. Anal. Chem. $\underline{75}$, 663-670.

Rappsilber, J., Mann, M. and Ishihama, Y. (2007). Protocol for micro-purification, enrichment, pre-fractionation and storage of peptides for proteomics using StageTips. Nat. Protoc. $\underline{2}$, 1896-1906.

Rauscher, S., Pacher, S., Hedtke, M., Kniemeyer, O. and Fischer, R. (2016). A phosphorylation code of the Aspergillus nidulans global regulator VelvetA (VeA) determines specific functions. Mol. Microbiol. 99, 909-924.

Reading, N.C. and Sperandio, V. (2006). Quorum sensing: The many languages of bacteria.

FEMS Microbiol. Lett. 254, 1-11.

Regulin, A. and Kempken, F., (2018). Fungal genotype determines survival of Drosophila melanogaster when competing with Aspergillus nidulans. PLoS One 13, e0190543. doi: 10.1371/journal.pone.0190543.

Rice, P., Longden, I. and Bleasby, A. (2000). EMBOSS: The European Molecular Biology Open Software Suite. Trends Genet. 16, 276-277.

Riquelme, M. (2013). Tip growth in filamentous fungi: A road trip to the apex. Annu. Rev. Microbiol. 67, 587-609.

Rodríguez-Urra, A.B., Jiménez, C., Nieto, M.I., Rodríguez, J., Hayashi, H. and Ugalde, U. (2012). Signaling the induction of sporulation involves the interaction of two secondary metabolites in Aspergillus nidulans. ACS Chem. Biol. $\underline{7}$, 599-606.

Rogers, S., Wells, R. and Rechsteiner, M. (1986). Amino acid sequences common to rapidly degraded proteins: the PEST hypothesis. Science 234, 364-368.

Rohs, R., Jin, X., West, S.M., Joshi, R., Honig, B. and Mann, R.S. (2010). Origins of specificity in protein-DNA recognition. Annu. Rev. Biochem. $\underline{79}$, 233-269.

Rojo, F. and Alonso, J.C. (1994). A novel site-specific recombinase encoded by the Streptococcus pyogenes plasmid pSM19035. J. Mol. Biol. 238, 159-172.

Rojo, F., Weise, F. and Alonso, J.C. (1993). Purification of the beta product encoded by the Streptococcus pyogenes plasmid pSM19035. A putative DNA recombinase required to resolve plasmid oligomers. FEBS Lett. $\underline{328}$, 169-173.

Romero-Calvo, I., Ocón, B., Martínez-Moya, P., Suárez, M.D., Zarzuelo, A., MartínezAugustin, O. and de Medina, F.S. (2010). Reversible Ponceau staining as a loading control alternative to actin in western blots. Anal. Biochem. 401, 318-320.

Romero, P., Obradovic, Z., Li, X., Garner, E.C., Brown, C.J. and Dunker, A.K. (2001). 
Sequence complexity of disordered protein. Proteins Struct. Funct. Genet. $42,38-48$. Ruger-Herreros, C., Rodríguez-Romero, J., Fernández-Barranco, R., Olmedo, M., Fischer, R.,

Corrochano, L.M. and Canovas, D. (2011). Regulation of conidiation by light in Aspergillus nidulans. Genetics $\underline{188}$, 809-822.

Saiki, R., Gelfand, D., Stoffel, S., Scharf, S., Higuchi, R., Horn, G., Mullis, K. and Erlich, H. (1988). Primer-directed enzymatic amplification of DNA with a thermostable DNA polymerase. Science 239 , 487-491.

Sanchez, J.F., Chiang, Y.M., Szewczyk, E., Davidson, A.D., Ahuja, M., Elizabeth Oakley, C., Woo Bok, J., Keller, N.P., Oakley, B.R. and Wang, C.C.C. (2010). Molecular genetic analysis of the orsellinic acid/F-9775 gene cluster of Aspergillus nidulans. Mol. BioSyst. 6, 587-593.

Sarikas, A., Hartmann, T. and Pan, Z.Q. (2011). The cullin protein family. Genome Biol. $\underline{12}$, 220. doi: 10.1186/gb-2011-12-4-220.

Sarikaya-Bayram, Ö., Bayram, Ö., Feussner, K., Kim, J.H., Kim, H.S., Kaever, A., Feussner, I., Chae, K.S., Han, D.M., Han, K.H. and Braus, G.H. (2014). Membrane-bound methyltransferase complex VapA-VipC-VapB guides epigenetic control of fungal development. Dev. Cell 29, 406-420.

Sarikaya-Bayram, Ö., Bayram, Ö., Valerius, O., Park, H.S., Irniger, S., Gerke, J., Ni, M., Han, K.H., Yu, J.H. and Braus, G.H. (2010). LaeA control of velvet family regulatory proteins for light-dependent development and fungal cell-type specificity. PLoS Genet. $\underline{6}$, e1001226. doi:10.1371/journal.pgen.1001226.

Sarikaya-Bayram, Ö., Palmer, J.M., Keller, N., Braus, G.H. and Bayram, Ö. (2015). One Juliet and four Romeos: VeA and its methyltransferases. Front. Microbiol. $\underline{6}$, doi: 10.3389/fmicb.2015.00001

Scherer, M. and Fischer, R. (1998). Purification and characterization of laccase II of Aspergillus nidulans. Arch. Microbiol. 170, 78-84.

Schindelin, J., Arganda-Carreras, I., Frise, E., Kaynig, V., Longair, M., Pietzsch, T., Preibisch, S., Rueden, C., Saalfeld, S., Schmid, B., Tinevez, J.Y., White, D.J., Hartenstein, V., Eliceiri, K., Tomancak, P. and Cardona, A. (2012). Fiji: An open-source platform for biological-image analysis. Nat. Methods $\underline{9}, 676-682$.

Schinke, J., Kolog Gulko, M., Christmann, M., Valerius, O., Stumpf, S.K., Stirz, M. and Braus, G.H. (2016). The DenA/DEN1 interacting phosphatase DipA controls septa positioning and phosphorylation-dependent stability of cytoplasmatic DenA/DEN1 during fungal development. PLoS Genet. 12, e1005949. doi: 10.1371/journal.pgen.1005949.

Schmidt, M.W., McQuary, P.R., Wee, S., Hofmann, K. and Wolf, D.A. (2009). F-Box-directed CRL complex assembly and regulation by the CSN and CAND1. Mol. Cell $\underline{49}, 1841-$ 1850. 
Schreiber, A. and Peter, M. (2014). Substrate recognition in selective autophagy and the ubiquitin-proteasome system. Biochim. Biophys. Acta 1843, 163-181.

Schroeckh, V., Scherlach, K., Nutzmann, H.W., Shelest, E., Schmidt-Heck, W., Schuemann, J., Martin, K., Hertweck, C. and Brakhage, A.A. (2009). Intimate bacterial-fungal interaction triggers biosynthesis of archetypal polyketides in Aspergillus nidulans. Proc. Natl. Acad. Sci. 106, 14558-14563.

Schultzhaus, Z., Yan, H. and Shaw, B.D. (2015). Aspergillus nidulans flippase DnfA is cargo of the endocytic collar and plays complementary roles in growth and phosphatidylserine asymmetry with another flippase, DnfB. Mol. Microbiol. $\underline{97}$, 18-32.

Schultzhaus, Z., Zheng, W., Wang, Z., Mouriño-Pérez, R. and Shaw, B. (2017). Phospholipid flippases DnfA and DnfB exhibit differential dynamics within the $A$. nidulans Spitzenkörper. Fungal Genet. Biol. $\underline{99}$, 26-28.

Schumacher, J., Simon, A., Cohrs, K.C., Traeger, S., Porquier, A., Dalmais, B., Viaud, M. and Tudzynski, B. (2015). The VELVET complex in the gray mold fungus Botrytis cinerea: Impact of BcLAE1 on differentiation, secondary metabolism, and virulence. Mol. PlantMicrobe Interact. 28, 659-674.

Schwechheimer, C., Serino, G., Callis, J., Crosby, W.L., Lyapina, S., Deshaies, R.J., Gray, W.M., Estelle, M. and Deng, X.W. (2001). Interactions of the COP9 signalosome with the E3 ubiquitin ligase SCFTIR1 in mediating auxin response. Science 292, 1379-1382.

Sebé-Pedrós, A., De Mendoza, A., Lang, B.F., Degnan, B.M. and Ruiz-Trillo, I. (2011). Unexpected repertoire of metazoan transcription factors in the unicellular holozoan Capsaspora owczarzaki. Mol. Biol. Evol. 28, 1241-1254.

Seo, J.A., Guan, Y. and Yu, J.H. (2003). Suppressor mutations bypass the requirement of fluG for asexual sporulation and sterigmatocystin production in Aspergillus nidulans. Genetics $165,1083-1093$.

Seo, J.A., Guan, Y. and Yu, J.H. (2006). FluG-dependent asexual development in Aspergillus nidulans occurs via derepression. Genetics 172, 1535-1544.

Sewall, T.C., Mims, C.W. and Timberlake, W.E., (1990). Conidium differentiation in Aspergillus nidulans wild-type and wet-white (wetA) mutant strains. Dev. Biol. $\underline{138}$, 499-508.

Shevchenko, A., Jensen, O.N., Podtelejnikov, A.V., Sagliocco, F., Wilm, M., Vorm, O., Mortensen, P., Shevchenko, A., Boucherie, H. and Mann, M. (1996). Linking genome and proteome by mass spectrometry: Large-scale identification of yeast proteins from two dimensional gels. Biochemistry $\underline{93}, 14440-14445$.

Shieh, S.Y., Ahn, J., Tamai, K., Taya, Y. and Prives, C. (2000). The human homologs of checkpoint kinases Chk1 and Cds1 (Chk2) phosphorylate, p53 at multiple DNA damageinducible sites. Genes Dev. 14, 289-300.

Shin, K.S., Kim, Y.H. and Yu, J.H. (2015). Proteomic analyses reveal the key roles of BrlA and 
AbaA in biogenesis of gliotoxin in Aspergillus fumigatus. Biochem. Biophys. Res. Commun. $\underline{463}, 428-433$.

Shlezinger, N., Irmer, H., Dhingra, S., Beattie, S.R., Cramer, R.A., Braus, G.H., Sharon, A. and Hohl, T.M., (2017). Sterilizing immunity in the lung relies on targeting fungal apoptosis-like programmed cell death. Science 1041, 1037-1041.

Sievers, F., Wilm, A., Dineen, D., Gibson, T.J., Karplus, K., Li, W., Lopez, R., McWilliam, H., Remmert, M., Söding, J., Thompson, J.D. and Higgins, D.G. (2011). Fast, scalable generation of high-quality protein multiple sequence alignments using Clustal Omega. Mol. Syst. Biol. ㄱ, doi: 10.1038/msb.2011.75.

Sigalov, A.B. (2016). Structural biology of intrinsically disordered proteins: Revisiting unsolved mysteries. Biochimie $\underline{125}, 112-118$.

Sigalov, A.B., Zhuravleva, A.V. and Orekhov, V.Y. (2004). Binding of intrinsically disordered proteins is not necessarily accompanied by a structural transition to a folded form. Biochimie 89, 419-421.

Skaar, J.R., Florens, L., Tsutsumi, T., Arai, T., Tron, A., Swanson, S.K., Washburn, M.P. and DeCaprio, J.A., (2007). PARC and CUL7 form atypical cullin RING ligase complexes. Cancer Res. 67, 2006-2014.

Skaar, J.R., Pagan, J.K. and Pagano, M. (2013). Mechanisms and function of subtrate recruitment by F-box proteins. Nat. Rev. Mol. Cell Biol. 14, 369-381.

Slesiona, S., Gressler, M., Mihlan, M., Zaehle, C., Schaller, M., Barz, D., Hube, B., Jacobsen, I.D. and Brock, M. (2012). Persistence versus escape: Aspergillus terreus and Aspergillus fumigatus employ different strategies during interactions with macrophages. PLoS One $\underline{7}$, e31223. doi: 10.1371/journal.pone.0031223.

Spencer, M.L., Theodosiou, M. and Noonan, D.J. (2004). NPDC-1, a novel regulator of neuronal proliferation, is degraded by the ubiquitin/proteasome system through a PEST degradation motif. J. Biol. Chem. 279, 37069-37078.

Staby, L., O'Shea, C., Willemoës, M., Theisen, F., Kragelund, B.B. and Skriver, K. (2017). Eukaryotic transcription factors: Paradigms of protein intrinsic disorder. Biochem. J. $\underline{474}$ 2509-2532.

Stefanowicz, K., Lannoo, N. and van Damme, E.J.M. (2015). Plant F-box proteins - judges between life and death. Crit. Rev. Plant Sci. 34, 523-552.

Stinnett, S.M., Espeso, E.A., Cobeño, L., Araújo-Bazán, L. and Calvo, A.M. (2007). Aspergillus nidulans VeA subcellular localization is dependent on the importin a carrier and on light. Mol. Microbiol. $\underline{63}, 242-255$.

Strauss, J. and Reyes-Dominguez, Y. (2011). Regulation of secondary metabolism by chromatin structure and epigenetic codes. Fungal Genet. Biol. 48, 62-69.

Suck, R.W.L. and Krupinska, K. (1996). Repeated probing of western blots obtained from 
Coomassie brilliant blue-stained or unstained polyacrylamide gels. Biotechniques $\underline{21}$, 418-422.

Suzuki, G., Yanagawa, Y., Kwok, S.F., Matsui, M. and Deng, X.W. (2002). Arabidopsis COP10 is a ubiquitin-conjugating enzyme variant that acts together with COP1 and the COP9 signalosome in repressing photomorphogenesis. Genes Dev. 16, 554-559.

Szewczyk, E., Chiang, Y.M.M., Oakley, C.E., Davidson, A.D., Wang, C.C.C. and Oakley, B.R. (2008). Identification and characterization of the asperthecin gene cluster of Aspergillus nidulans. Appl. Environ. Microbiol. 74, 7607-7612.

Szewczyk, E., Oakley, B.R., Nayak, T., Oakley, C.E., Edgerton, H., Xiong, Y., Taheri-Talesh, N. and Osmani, S.A. (2006). Fusion PCR and gene targeting in Aspergillus nidulans. Nat. Protoc. 1, 3111-3120.

Szilágyi, M., Kwon, N.J., Bakti, F., M-Hamvas, M., Jámbrik, K., Park, H., Pócsi, I., Yu, J.H. and Emri, T. (2011). Extracellular proteinase formation in carbon starving Aspergillus nidulans cultures - physiological function and regulation. J. Basic Microbiol. 51, 625-634.

Takeshita, N., Manck, R., Grün, N., de Vega, S.H. and Fischer, R. (2014). Interdependence of the actin and the microtubule cytoskeleton during fungal growth. Curr. Opin. Microbiol. $\underline{20}, 34-41$.

Tao, Z., Fusco, A., Huang, D. Bin, Gupta, K., Young Kim, D., Ware, C.F., Van Duyne, G.D. and Ghosh, G. (2014). p100/lkBס sequesters and inhibits NF-kB through kappaBsome formation. Proc. Natl. Acad. Sci. 111, 15946-15951.

Teyra, J., Huang, H., Jain, S., Guan, X., Dong, A., Liu, Y., Tempel, W., Min, J., Tong, Y., Kim, P.M., Bader, G.D. and Sidhu, S.S. (2017). Comprehensive analysis of the human SH3 domain family reveals a wide variety of non-canonical specificities. Structure $\underline{25}, 1598$ 1610.

Timpner, C., Braus-Stromeyer, S.A., Tran, V.T. and Braus, G.H. (2013). The Cpc1 regulator of the cross-pathway control of amino acid biosynthesis is required for pathogenicity of the vascular pathogen Verticillium longisporum. Mol. Plant-Microbe Interact. 26, 13121324.

Towbin, H., Staehelin, T. and Gordon, J. (1979). Electrophoretic transfer of proteins from polyacrylamide gels to nitrocellulose sheets: Procedure and some applications. Proc. Natl. Acad. Sci. $\underline{76}$, 4350-4354.

Tsitsigiannis, D.I., Kowieski, T.M., Zarnowski, R. and Keller, N.P. (2005). Three putative oxylipin biosynthetic genes integrate sexual and asexual development in Aspergillus nidulans. Microbiology 151, 1809-1821.

Tsitsigiannis, D.I., Zarnowski, R. and Keller, N.P. (2004). The lipid body protein, PpoA, coordinates sexual and asexual sporulation in Aspergillus nidulans. J. Biol. Chem. 279, 11344-11353. 
Tsui, R., Kearns, J.D., Lynch, C., Vu, D., Ngo, K., Basak, S., Ghosh, G. and Hoffmann, A. (2015). IKB $\beta$ enhances the generation of the low-affinity NFKB/RelA homodimer. Nat. Commun. $\underline{6}$, doi:10.1038/ncomms8068.

Tyanova, S., Temu, T., Sinitcyn, P., Carlson, A., Hein, M., Geiger, T., Mann, M. and Cox, J. (2016). The Perseues computational platform for comprehensive analysis of (prote)omics data. Nature Meth. $\underline{13}$, 731-740.

Uehling, J., Deveau, A. and Paoletti, M. (2017). Do fungi have an innate immune response? An NLR-based comparison to plant and animal immune systems. PLoS Pathog. 13, e1006578. doi: 10.1371/journal.ppat.1006578.

Ugalde, U. and Rodriguez-Urra, A.B. (2016). Autoregulatory signals in mycelial fungi. In: Wendland, J. (Ed.), The Mycota I: Growth, Differentiation and Sexuality. Springer International Publishing Switzerland 2016, Cham, pp. 203-213.

Untergasser, A., Cutcutache, I., Koressaar, T., Ye, J., Faircloth, B.C., Remm, M. and Rozen, S.G. (2012). Primer3-new capabilities and interfaces. Nucleic Acids Res. $\underline{40}$, e115. doi:10.1093/nar/gks596.

Uversky, V.N., (2003). A protein-chameleon: Conformational plasticity of a-synuclein, a disordered protein involved in neurodegenerative disorders. J. Biomol. Struct. Dyn. 21, 211-234.

Uversky, V.N. (2013). Unusual biophysics of intrinsically disordered proteins. Biochim. Biophys. Acta 1834, 932-951.

Uversky, V.N. (2015). Functional roles of transiently and intrinsically disordered regions within proteins. FEBS J. 282, 1182-1189.

Uversky, V.N., Gillespie, J.R. and Fink, A.L. (2000). Why are "natively unfolded" proteins unstructured under physiologic conditions? Proteins Struct. Funct. Genet. 41, 415-427. van der Lee, R., Lang, B., Kruse, K., Gsponer, J., de Groot, N.S., Huynen, M.A., Matouschek, A., Fuxreiter, M. and Babu, M.M. (2014). Intrinsically disordered segments affect protein half-life in the cell and during evolution. Cell Rep. $\underline{8}, 1832-1844$.

von Zeska Kress, M.R., Harting, R., Bayram, Ö., Christmann, M., Irmer, H., Valerius, O., Schinke, J., Goldman, G.H. and Braus, G.H. (2012). The COP9 signalosome counteracts the accumulation of cullin SCF ubiquitin E3 RING ligases during fungal development. Mol. Microbiol. $\underline{\text { 83, }}$ 1162-1177.

Vuzman, D. and Levy, Y. (2012). Intrinsically disordered regions as affinity tuners in proteinDNA interactions. Mol. BioSyst. $\underline{8}, 47-57$.

Wagner, E.F. (2001). AP-1 - Introductory remarks. Oncogene 20, 2334-2335.

Wang, B., Lv, Y., Li, X., Lin, Y., Deng, H. and Pan, L. (2017). Profiling of secondary metabolite gene clusters regulated by LaeA in Aspergillus niger FGSC A1279 based on genome sequencing and transcriptome analysis. Res. Microbiol. pii: S0923-2508(17)30168-7. 
doi: 10.1016/j.resmic.

Wang, W., Wu, D., Pan, H. and Turgeon, B.G. (2014). Vel2 and Vos1 hold essential roles in ascospore and asexual spore development of the heterothallic maize pathogen Cochliobolus heterostrophus. Fungal Genet. Biol. 0ㅡ, 113-124.

Wanke, C., Eckert, S., Albrecht, G., van Hartingsveldt, W., Punt, P.J., van den Hondel, C.A. and Braus, G.H. (1997). The Aspergillus niger GCN4 homologue, $c p c A$, is transcriptionally regulated and encodes an unusual leucine zipper. Mol. Microbiol. 23 , 23-33.

Ward, J.J., McGuffin, L.J., Bryson, K., Buxton, B.F. and Jones, D.T. (2004a). The DISOPRED server for the prediction of protein disorder. Bioinformatics 20, 2138-2139.

Ward, J.J., Sodhi, J.S., McGuffin, L.J., Buxton, B.F. and Jones, D.T. (2004b). Prediction and functional analysis of native disorder in proteins from the three kingdoms of life. J. Mol. Biol. $\underline{337}, 635-645$.

Weatheritt, R.J., Luck, K., Petsalaki, E., Davey, N.E. and Gibson, T.J. (2012). The identification of short linear motif-mediated interfaces within the human interactome. Bioinformatics 28, 976-982.

Webster, R.H. and Sil, A. (2008). Conserved factors Ryp2 and Ryp3 control cell morphology and infectious spore formation in the fungal pathogen Histoplasma capsulatum. Proc. Natl. Acad. Sci. 105, 14573-14578.

Wells, M., Tidow, H., Rutherford, T.J., Markwick, P., Jensen, M.R., Mylonas, E., Svergun, D.I., Blackledge, M. and Fersht, A.R. (2008). Structure of tumor suppressor p53 and its intrinsically disordered N-terminal transactivation domain. Proc. Natl. Acad. Sci. $\underline{105}$ 5762-5767.

Wiemann, P., Brown, D.W., Kleigrewe, K., Bok, J.W., Keller, N.P., Humpf, H. and Tudzynski, B. (2010). FfVel1 and FfLae1, components of a velvet-like complex in Fusarium fujikuroi, affect differentiation, secondary metabolism and virulence. Mol. Microbiol. 77, 972-994.

Wieser, J., Lee, B.N., Fondon, J.W. and Adams, T.H. (1994). Genetic requirements for initiating asexual development in Aspergillus nidulans. Curr. Genet. 27, 62-69.

Wingender, E. (1997). Classification scheme of eukaryotic transcription factors. Mol. Biol. $\underline{31}$ 584-600.

Wongsuk, T., Pumeesat, P. and Luplertlop, N. (2016). Fungal quorum sensing molecules: Role in fungal morphogenesis and pathogenicity. J. Basic Microbiol. 56, 440-447.

Wright, P.E. and Dyson, H.J. (2015). Intrinsically disordered proteins in cellular signalling and regulation. Nat. Rev. Mol. Cell Biol. 16, 18-29.

Wu, Y., Xu, L., Yin, Z., Dai, Q., Gao, X., Feng, H., Voegele, R.T. and Huang, L. (2017). Two members of the velvet family, $V m V e A$ and $V m V e l B$, affect conidiation, virulence and pectinase expression in Valsa mali. Mol. Plant. Pathol. doi: 10.1111/mpp.12645. 
Yadav, S., Kalra, N., Ganju, L. and Singh, M. (2017). Activator protein-1 (AP-1): A bridge between life and death in lung epithelial (A549) cells under hypoxia. Mol. Cell. Biochem. $\underline{436}, 99-110$.

Yager, L.N., (1992). Early developmental events during asexual and sexual sporulation in Aspergillus nidulans. Biotechnology 23, 19-41.

Yin, W.B., Reinke, A.W., Szilagyi, M., Emri, T., Chiang, Y.M., Keating, A.E., Pocsi, I., Wang, C.C.C. and Keller, N.P. (2013). bZIP transcription factors affecting secondary metabolism, sexual development and stress responses in Aspergillus nidulans. Microbiology 159, 77-88.

Yu, J., Cleveland, T.E., Nierman, W.C. and Bennett, J.W. (2005). Aspergillus flavus genomics: Gateway to human and animal health, food safety, and crop resistance to diseases. Rev. Iberoam. Micol. 22, 194-202.

Yu, J.H. (2010). Regulation of Development in Aspergillus nidulans and Aspergillus fumigatus. Mycobiology $\underline{38}, 229-237$.

Yu, J.H. and Keller, N. (2005). Regulation of secondary metabolism in filamentous fungi. Annu. Rev. Phytopathol. $\underline{43}$, 437-458.

Yu, J.H., Kwon, N.J., Shin, K.S., Gao, N. and Ni, M. (2010). Regulation of Aspergillus conidiation. In: Borkovich, K.A., Ebbole, D.J. (Eds.), Cellular and Molecular Biology of Filamentous Fungi. ASM Press, Washington, pp. 559-576.

Zaffagnini, G. and Martens, S. (2016). Mechanisms of selective autophagy. J. Mol. Biol. $\underline{428}$, 1714-1724. 


\section{List of abbreviations}

\begin{tabular}{|c|c|}
\hline$\%$ & Percent \\
\hline${ }^{\circ} \mathrm{C}$ & Degree Celsius \\
\hline$\Delta$ & Deletion \\
\hline$\mu \mathrm{g}$ & Microgram \\
\hline$\mu \mathrm{L}$ & Microliter \\
\hline$\mu \mathrm{m}$ & Micrometer \\
\hline aa & Amino acids \\
\hline ADP & Adenosine diphosphate \\
\hline AspGD & Aspergillus genome database \\
\hline ATP & Adenosine triphosphate \\
\hline BLAST & Basic local alignment search tool \\
\hline bp & Base pairs \\
\hline bZIP & Leucine zipper domain \\
\hline $\mathrm{C} 2 \mathrm{H} 2$ & $\mathrm{Cys}_{2} \mathrm{His}_{2}$ zinc cluster fungal type DNA binding domain \\
\hline CADRE & Central Aspergillus repository \\
\hline cDNA & Complementary DNA \\
\hline CDP & Central developmental pathway \\
\hline Co-IP & Co-immunoprecipitation \\
\hline COP9 & Constitutive photomorphogenic 9 \\
\hline CRL & Cullin-RING ligase \\
\hline CSN & COP9 signalosome \\
\hline C-terminus & Carboxy terminus \\
\hline$d$ & $\operatorname{Day}(\mathrm{s})$ \\
\hline DAPI & 4',6-diamidino-2-phenylindole \\
\hline DBD & DNA binding domain \\
\hline DIC & Differential interference contrast \\
\hline DNA & Deoxyribonucleic acid \\
\hline DTT & Dithiothreitol \\
\hline
\end{tabular}

EDTA Ethylenediaminetetraacetic acid

e.g. Exempli gratia $=$ for example

ER Endoplasmatic reticulum

ERAD Endoplasmatic reticulum associated degradation

g Gram

GFP Green fluorescent protein

GTP Guanosin triphosphate 


\begin{tabular}{|c|c|}
\hline h & Hour(s) \\
\hline $\mathrm{H} 2 \mathrm{~A}$ & Histone \\
\hline $\mathrm{dH}_{2} \mathrm{O}$ & Deionized $\mathrm{H}_{2} \mathrm{O}$ \\
\hline HPLC & High performance liquid chromatography \\
\hline IDD & Intrinsically disordered domain \\
\hline $\mathrm{kb}$ & Kilo bases \\
\hline kDa & Kilo Dalton \\
\hline $\mathrm{L}$ & Liter \\
\hline LB & Lysogen broth \\
\hline LC & Liquid chromatography \\
\hline M & Molar (mol/L) \\
\hline mAbs & Milli absorbance units \\
\hline MAP & Mitogen-activated (kinase) \\
\hline $\mathrm{mg}$ & Milligram \\
\hline $\min$ & Minute(s) \\
\hline $\mathrm{mL}$ & Milliliter \\
\hline MM & Minimal medium \\
\hline $\mathrm{mM}$ & Millimolar \\
\hline mRNA & Messenger RNA \\
\hline MS/MS & Tandem mass spectrometry \\
\hline natR & Nourseothricin resistance marker \\
\hline natRM & Nourseothricin recyclable resistance marker \\
\hline NES & Nuclear export signal \\
\hline NLR & NOD-like receptor \\
\hline NLS & Nuclear localization signal \\
\hline $\mathrm{N}$-terminal & Amino-terminal \\
\hline $\mathrm{o} / \mathrm{n}$ & Over night \\
\hline ORF & Open reading frame \\
\hline$P$ & Promoter \\
\hline p.c. & Personal communication \\
\hline PCR & Polymerase chain reaction \\
\hline phleoR & Phleomycin resistance marker \\
\hline phleoRM & Phleomycin recyclable resistance marker \\
\hline PKS & Polyketide synthase \\
\hline Psi-factor & Precocious sexual inducer-factor \\
\hline PTM & Posttranslational modification \\
\hline qRT-PCR & Quantitative real-time PCR \\
\hline
\end{tabular}




$\begin{array}{ll}\text { R } & \text { Resistance } \\ \text { RHD } & \text { Rel homology domain } \\ \text { RING } & \text { Really interesting new gene } \\ \text { RNA } & \text { Ribonucleic acid } \\ \text { ROS } & \text { Reactive oxygen species } \\ \text { rRNA } & \text { Ribosomal RNA } \\ \text { rpm } & \text { Rounds per minute } \\ \text { rt } & \text { Room temperature } \\ \text { SCF complex } & \text { SkpA-Cullin-F-box complex } \\ \text { SEM } & \text { Standard error of the mean } \\ \text { SLiM } & \text { Short Linear (peptide) Motif } \\ \text { SDS } & \text { Sodium dodecyl sulfate } \\ \text { spp. } & \text { Species } \\ \text { t } & \text { Terminator } \\ \text { TAD } & \text { Transcription activation domain } \\ \text { TF } & \text { Transcription factor } \\ \text { TLC } & \text { Thin layer chromatography } \\ \text { tRNA } & \text { Transfer RNA } \\ \text { UDA } & \text { Upstream developmental activator } \\ \text { UPS } & \text { Ubiquitin proteasome system } \\ \text { v/v } & \text { Volume per volume } \\ \text { w/v } & \text { Weight per volume } \\ \text { wt } & \text { Wildtype } \\ & \end{array}$




\section{Table of figures}

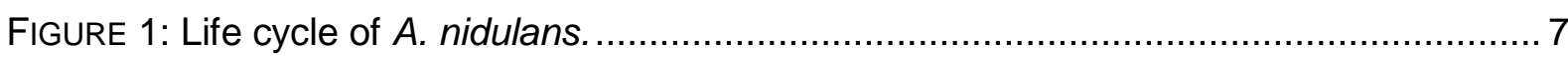

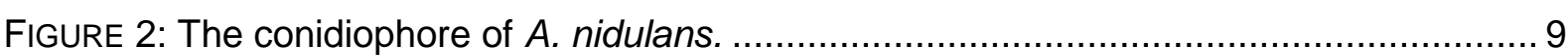

FIGURE 3: The regulatory network of $A$. nidulans velvet proteins. .................................... 16

FIGURE 4: Domain architecture of $A$. nidulans velvet proteins. ...................................... 19

FIGURE 5: The VosA ${ }_{1-190}$ velvet domain crystal structure is similar to the NF-kB Rel homology domain structure.

FIGURE 6: The VelB intrinsically disordered domain is absent in the crystal structure of the VelB-VosA ${ }_{1-190}$ heterodimer of $A$. nidulans.

FIGURE 7: Most of eukaryotic proteins contain structured domains und intrinsically disordered regions 23

FIGURE 8: Protein degradation is achieved by different mechanisms in eukaryotes. 24

FIGURE 9: The ubiquitin proteasome pathway. 26

FIGURE 10: The SkpA-CullinA-F-box complex of $A$. nidulans. 27

FIGURE 11: The F-box domain contains a highly conserved proline and leucine residue. 29

FIGURE 12: Schematic illustration of integration and recycling of a recyclable marker cassette.

FIGURE 13: Ascomycetes share a conserved motif in the VelB intrinsically disordered domain. 64

FIGURE 14: The amino acid sequence of the $A$. nidulans VelB IDD differs from the IDD of representatives of the Basidiomycota.

FIGURE 15: The VelB subcellular localization is independent of the IDD in A. nidulans. 67

FIGURE 16: The VeIB IDD alters selective heterodimer formation.

FIGURE 17: The VelB IDD is required for co-immunoprecipitation of the VelB-VosA heterodimer. 73

FIGURE 18: VelB binds to the velvet domain of VosA in vitro independently of the IDD....... 75

FIGURE 19: Affinity chromatography of VelB-His and VelB ${ }^{I D D} \Delta_{-H}$ His fusion proteins................ 76

FIGURE 20: Homodimer formation of VelB is dependent on the IDD in vitro.

FIGURE 21: The VeIB IDD is dispensable for conidiospore formation in hyphae growing from a single spot in $A$. nidulans.

FIGURE 22: The IDD of VelB is required for efficient asexual spore formation within aging mycelium in $A$. nidulans.

FIGURE 23: Cleistothecium formation and maturation is independent of the VelB IDD......... 81

FIGURE 24: The VelB IDD is dispensable for conidiospore viability .................................... 83

FIGURE 25: Accurate regulation of sterigmatocystin production requires the VelB IDD........ 84

FIGURE 26: Secondary metabolite production is altered in the vel$B^{I D D_{\Delta}}$ mutant. .................. 85 
FIGURE 27: Sterigmatocystin production is decreased in the velB deletion mutant. 86

FIGURE 28: The VelB IDD promotes VelB-VosA heterodimer formation and regulates secondary metabolite production.

FIGURE 29: The relative expression of velB is not altered in a velB ${ }^{I D D_{\Delta}}:$. sgfp expressing strain compared to a velB::sgfp expressing strain. 91

FIGURE 30.: The IDD destabilizes VelB-GFP during fungal development. .93

FIGURE 31: The IDD destabilizes VelB-GFP 94

FIGURE 32: Investigation of the relative amount of VelB in fbox deletion mutants in A. nidulans.

FIGURE 33: Examination of the relative amount of VelB in 72 fbox deletion strains of

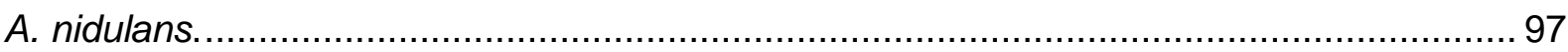

FIGURE 34: VelB protein level is significantly increased in the fbox $18 \Delta$ mutant..................... 98

FIGURE 35: The stability of VelB is dependent on fbox18 in A. nidulans. ............................. 99

FIGURE 36: Phenotypical analysis of $f b x 18 \Delta$ compared to wildtype. 100

FIGURE 37: The VeIB IDD of $A$. nidulans is enriched in proline, serine and tyrosine residues. 103

FIGURE 38: Predictions of phosphorylated amino acids of $A$. nidulans VelB. ..................... 105

FIGURE 39: The VelC-GFP fusion protein is functional in $A$. nidulans............................... 106

FIGURE 40: VelC-GFP is unstable and contains a PEST domain. ................................ 107

FIGURE 41: Conidiospore formation is delayed in the velB ${ }^{I D D_{\Delta}} / v^{2} / C \Delta$ mutant. .................... 111

FIGURE 42: The velB ${ }^{I D R_{\Delta}} / v e l C \Delta$ double mutant is delayed in secondary metabolite production. 113

FIGURE 43: Sterigmatocystin production is increased in the $v e l B^{I D D} /$ velC $\Delta$ double mutant. 114 FIGURE 44: The velB $\Delta /$ velC $\Delta$ double deletion mutant partially rescues the velB $\Delta$ phenotype.

FIGURE 45: The velB/velC double deletion mutant produces more conidiospores than the wildtype. 117

FIGURE 46: $A$. nidulans is viable without the four velvet encoding genes. 118

FIGURE 47: Selective heterodimer formation is supported by the VelB IDD in A. nidulans. . 123

FIGURE 48: Phylogenetic position of Capsaspora owczarzaki. 125

FIGURE 49: Intrinsic disorder prediction in comparison to $A$. nidulans VelB. 127

FIGURE 50: Substrate recruitment mechanisms of F-box proteins. 136

FIGURE 51: Comprehensive model of the consequences of VelB selective dimer formation in $A$. nidulans. 


\section{List of tables}

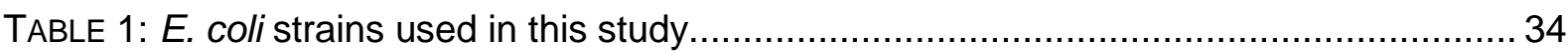

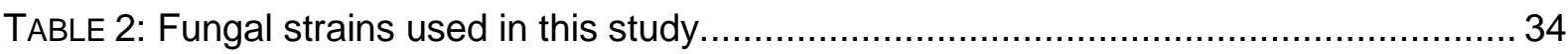

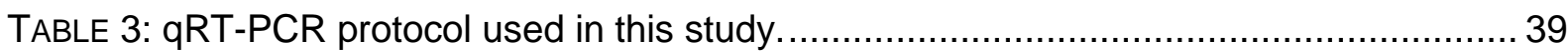

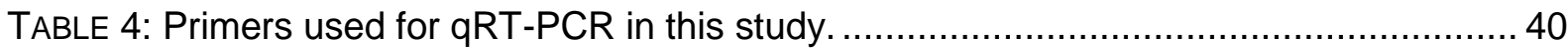

TABLE 5: Plasmids constructed and used in this study. ................................................. 42

TABLE 6: Oligonucleotides used for amplifications, plasmid constructions and sequencing. . 42

TABLE 7: Putative interaction partners identified in the GFP pull down of VelB-GFP and

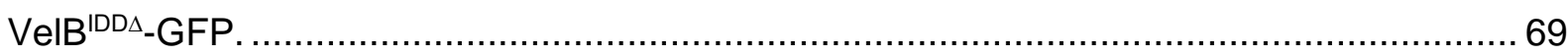

TABLE 8: List of secondary metabolites identified via LC-MS analyses. ............................. 89

TABLE 9: Specific dimer formation of velvet domain protein results in regulation of secondary metabolite gene clusters in $A$. nidulans.................................................................. 90

TABLE 10: Short linear motifs of the VelB intrinsically domain of $A$. nidulans. ..................... 102

TABLE 11: Putative interaction partners of $A$. nidulans VelC during early sexual development. 


\section{Acknowledgements}

First and foremost, I would like to express my sincere gratitude to Prof. Dr. Gerhard H. Braus for his constant support and outstanding supervision during my time as a $\mathrm{PhD}$ student in his department. I am deeply grateful for his encouragement, excellent mentoring and advisement, including many fruitful and inspiring discussions. I also appreciate the frequent chances to attend at national and international conferences.

I also want to thank Prof. Dr. Ralf Ficner for being member of my thesis committee. I had the chance to do a lab rotation during my Master studies in his department and I am very thankful for his expertise and helpful discussions also during my time as a PhD student.

I am profoundly thankful to Prof. Dr. Stefanie Pöggeler as a member of my thesis committee for constructive discussions, ideas and suggestions for my project during my doctoral studies.

Furthermore, I thank Prof. Dr. Heike Krebber, Prof. Dr. Rolf Daniel and Jun. Prof. Dr. Kai Heimel for being members of my examination board.

I am grateful to conduct my PhD studies as a member of the doctoral program "Microbiology and Biochemistry" of the Göttingen Graduate School for Neuroscience, Biophysics and Molecular Biosciences (GGNB). Being member of the GGNB was a great opportunity for scientific learning and exchanging with other researchers. I especially enjoyed the excellent soft skill courses, which were offered from the GGNB and were very helpful for me. I am also very thankful to GGNB for financial support in means of providing travel grants.

A big word of thanks goes to Dr. Jennifer Gerke and Dr. Christoph Sasse for their magnificent supervision, their constant help and encouragement during my research. Their practical tips and suggestions during our regular meetings are deeply appreciated. I also want to thank them for proof reading of my thesis.

Special thanks go to Dr. Jennifer Gerke for the HPLC measurements for my work and for the great time we had at Asilomar and for her constant encouragement.

I want to thank Dr. Oliver Valerius and Dr. Kerstin Schmitt for performing the LC-MS/MS measurements and for the helpful discussions regarding the results. I also thank them for reading and correcting the LC-MS/MS part of the material and methods section in this work! Moreover, I want to thank Dr. Achim Dickmanns for fruitful discussions and suggestions and Dr. Piotr Neumann for this help regarding the superposition of crystal structures for my thesis. 
Furthermore, I want to thank Prof. Dr. Helge B. Bode from the Goethe-University Frankfurt for the possibility to identify the secondary metabolites found in my samples. I am thankful to Dr. Antje K. Heinrich and for doing the LC-MS measurements and for helping me with the evaluation of the data and with writing the section in the material and methods part.

I also want to thank Dr. Rebekka Harting for valuable discussions during my studies and for proof reading of my thesis. Additionally, I want to thank Annalena Höfer for providing gDNA of V. dahliae.

I am thankful to Dr. Blagovesta Popova for her help regarding quantification of my data and working with the StrepTactin beads.

Moreover, I would like to thank Heidi Northemann, Nicole Scheiter, Gabriele Heinrich and Andrea Wäge for the administrative and technical assistance.

I want to thank all former and current members of my lab 1.102 and of the Department of Molecular Microbiology and Genetics for the nice and friendly working atmosphere. I really enjoyed the time doing my PhD studies with all of you. I also want to thank the members of AG Pöggeler and AG Heimel for their open ears and help.

Especially, I want to thank Verena Grosse for technical and mental support and Anja Abelmann for proof reading. I really enjoy the work with you two on the "girls side" of the lab. I want to thank Anna Maria Köhler for her help and advice for recombinant expression and purification experiments and Cindy Meister for helping me with the evaluation of the LC-MS/MS data employing the Perseus program. I also thank them and additionally Fruzsina Bakti for many helpful discussions, proof reading of my thesis and for constantly encouraging me mentally and emotionally. I am deeply grateful for our close friendship and our great time in- and outside of the lab. I am looking forward to kiss, as prophesied, the Gänseliesel together with Anna and Cindy. :-) My thanks also go to the Ch/Kristians for their friendship.

I am deeply grateful to Dr. Alexandra Kleinknecht and for the time we spend in- and outside the lab. She always had an open ear and encouraged me in many ways. Thank you so much for your support and for proof reading of my thesis! Additionally, I thank her husband Dr. Alexander Kleinknecht, who made Alex ${ }^{2}$ complete. I enjoy the great and pleasant time we have together.

Besides of my friends with whom I am privileged to work with, I also want to thank my close friends from outside of the lab for all their support. Big thanks go to the "Bachelor Mädels", who 
I know since almost the first day of my studies in Göttingen. I enjoy our weekly and delicious girls night and although we go our separate ways I know that our strong connection will never fall apart.

I am grateful to my "Eckernförde crew", Miri, Dennis, Siva and their partners David, Alex and Anke. Although we do not see each other that often I know that I can always count on you and that our friendship lasts forever!

Last but not least, my biggest thanks go to my family, especially to my parents and for their constant support and love. Without you this would never have been possible! I also want to thank my sister Stefanie. Although the Atlantic Ocean lies between us, I know that you are always there for me!

Above all, my thanks go to my husband Karl. Thank you so much for your professional and more important emotional support. There are no words sufficient to describe how thankful I am to have you on my side - in the lab and at home ;-). I love you and I am looking forward to our common future! 


\title{
Curriculum vitae
}

Personal details:

\author{
Sabine Thieme (née Reen) \\ born on May $28^{\text {th }}, 1988$ \\ in Eckernförde, Germany
}

Higher education and academic qualification

$02 / 2014-2018$

$10 / 2011-11 / 2013$

$10 / 2007-09 / 2011$

Scientific teaching

$08 / 2016-09 / 2016$

$10 / 2016-10 / 2016$

$01 / 2017-01 / 2017$
PhD student in the doctoral program "Microbiology and Biochemistry" of the Göttingen Graduate School of Neurosciences, Biophysics and Molecular Biosciences (GGNB) at the Georg-August-Universität Göttingen, Germany.

$\mathrm{PhD}$ thesis at the Institute for Microbiology and Genetics, Department of Molecular Microbiology and Genetics (Prof. Dr. Gerhard Braus). Title: "Insertion of an intrinsically disordered domain in VelB supports selective heterodimer formation of fungal velvet domain regulatory proteins in Aspergillus nidulans"

Master of Science in Microbiology and Biochemistry at the Georg-August-Universität Göttingen, Germany.

Master thesis at the Institute for Microbiology and Genetics, Department of Molecular Microbiology and Genetics (Prof. Dr. Gerhard Braus). Title: "Interaction partners of F-box protein in Aspergillus nidulans"

Bachelor of Science in Biology at the Georg-AugustUniversität Göttingen, Germany.

Bachelor thesis at the Albert-von-Haller Institute for Plant Sciences, Department of Plant Biochemistry (Prof. Dr. Ivo Feussner). Title: "Psi-Faktoren in Verticillium longisporum"

Supervision of a laboratory internship of the Master student, Ezgi Akidil

Supervision of a laboratory internship of the Master student, Kiana Mohajeri

Supervision of a three weeks Master practical course 


\section{Publications}

2018

Karl G. Thieme, Jennifer Gerke, Christoph Sasse, Oliver Valerius, Sabine Thieme, Antje K. Heinrich, Helge B. Bode, Arthur F. J. Ram and Gerhard H. Braus (2018). The Zinc cluster transcription factor ZtfA is an activator of asexual development and secondary metabolism and regulates the oxidative stress response in the filamentous fungus Aspergillus nidulans. In revision.

\section{Further scientific and work experiences}

$01 / 2015-05 / 2015$

$12 / 2011-03 / 2012$

07-09/2007-2012

School education

$1998-2007$

$1994-1998$
Scientific assistant at the Max Planck Institute for Biophysical Chemistry Göttingen, Germany, in the research group Chromatin Biochemistry (Prof. Dr. Wolfgang Fischle).

Scope of duty: Purification of plasmid DNA in high amounts.

Scientific assistant at the Georg-August-Universität Göttingen, Germany at the Albert-von-Haller Institute for Plant Sciences, Department of Plant Biochemistry (Prof. Dr. Ivo Feussner).

Scope of duty: Preparation of Arabidopsis thaliana seeds for further experiments.

Agricultural assistant at the Pioneer Hi-Bred Northern Europe Service Division $\mathrm{GmbH}$ in Wulfhagen, Germany.

Scope of duty: Assistance in the execution of rape seed breeding experiments.

High school and attainment of the university-entrance diploma (Abitur) at the Jungmannschule Eckernförde

Elementary school at the Fritz-Reuter Schule, Eckernförde 